FINITE ELEMENT MODELLING OF THIN SHEET STEEL SCREW CONNECTIONS

\author{
E. RAUTENBACH
}




\section{FINITE ELEMENT MODELLING OF THIN SHEET STEEL SCREW CONNECTIONS}

\section{ELANA RAUTENBACH}

A dissertation submitted in partial fulfilment of the requirements for the degree of MASTER OF ENGINEERING (STRUCTURAL ENGINEERING) in the

FACULTY OF ENGINEERING, BUILT-ENVIRONMENT AND INFORMATION TECHNOLOGY

\section{UNIVERSITY OF PRETORIA}

November 2016 


\title{
DISSERTATION SUMMARY
}

\section{FINITE ELEMENT MODELLING OF THIN SHEET STEEL SCREW CONNECTIONS}

\section{E. RAUTENBACH}

\author{
Supervisor: $\quad$ Professor BWJ van Rensburg \\ Department: Civil Engineering \\ University: University of Pretoria \\ Degree: $\quad$ Master of Engineering (Structural Engineering)
}

Presently, there is only a limited body of research regarding the numerical investigation of screwed connections, as opposed to the analysis of bolted connections. This trend can be attributed to the difficulty in attaining convergence when the steel sheet thickness is less than $1 \mathrm{~mm}$, in conjunction with complications in modelling the thread on the screw shank, as well as the uncertainty of the contact settings between the screws and plates. This study focussed on the non-linear finite element modelling procedure, utilising the explicit dynamic analysis technique, and how the accuracy is affected by the various modelling parameters.

The objective was to investigate the different modalities of finite element modelling to effectively analyse thin sheet steel screwed connections, through calibration of the obtained results against the test data found within existing literature. Several aspects of the modelling procedure were examined, including: contact definitions when using kinematic or penalty contact tracking; the weighting between the master and slave surfaces in the contact formulation; and the effective control of the applied load and how it affects the energy output of the finite element model.

The finite element model was verified through calibration against the research conducted by Yan (2012), and the decision was made to limit the application of the displacement controlled loading, up to a maximum of $5 \mathrm{~mm}$. The finite element analysis results obtained remained within the $5 \%$ top and bottom curves obtained by Yan (2012)

A Von Mises stress plot of the side profile for the verified model was presented where it was observed that as the load is applied, the screw rotates, and follows the archetypical bearing/tilting mode of failure. This was further verified through examination of the area where the material was yielding using the equivalent plastic strain (PEEQ), which was defined as the total accumulation of plastic strain to define the yield surface size. 
The final calibrated model was then utilised in subsequent parametric studies, which investigated the effect of the steel sheet thickness, as well as the number, pattern and size of the screws used. Results obtained through finite element analysis were further compared to the relevant design standards (AISI S100, 2007; AS/NZS 4600, 2005; EC3, 2006). In this analysis the model was only pulled to a maximum displacement of $5 \mathrm{~mm}$, hence ultimate load for this comparison was defined to be that of a deflection based load. Hence, deflection based load was defined as the maximum load at/or before a screw hole deformation of $3 \mathrm{~mm}$.

The results of the parametric study indicated that the current connection provisions set out in the Eurocode 3 (2006) Design Standards can be used to effectively predict the mode of failure of screwed connections at deflection based load level. In only a limited number of cases did the AISI (AISI S100, 2007) and AS/NZS (2005) Design Standards result in incorrect failure mode predictions. Results concluded that the finite element model could effectively simulate the expected experimental behaviour of these connections. 


\title{
FINITE ELEMENT MODELLING OF THIN SHEET STEEL SCREW CONNECTIONS
}

\section{E. RAUTENBACH}

\author{
Supervisor: $\quad$ Professor BWJ van Rensburg \\ Department: Civil Engineering \\ University: University of Pretoria \\ Degree: $\quad$ Master of Engineering (Structural Engineering)
}

This study focused on the non-linear finite element modelling procedure, utilising the explicit dynamic analysis technique, and how the various modelling parameters affect the accuracy. The computer modelling of screwed connections is a complex task, and the objective was to investigate the different modalities of finite element modelling to effectively analyse thin sheet steel screwed connections, through calibration against test data found within existing literature. Several aspects of the modelling procedure were examined, including contact definitions, the effective control of the applied load and how it affects the energy output of the finite element model. The final calibrated model was utilised in subsequent parametric studies, which investigated the effect of the steel sheet thickness, as well as the number of the screws used. Results concluded that the finite element model could effectively simulate the expected experimental behaviour of these connections. 


\section{DECLARATION}

I, the undersigned hereby declare that:

I understand what plagiarism is and I am aware of the University's policy in this regard;

The work contained in this dissertation is my own original work;

I did not refer to work of current or previous students, lecture notes, handbooks or any other study material without proper referencing;

I have not allowed anyone to copy any part of my dissertation;

I have not previously in its entirety or in part submitted this dissertation at any university for a degree.

\section{Disclaimer:}

The work presented in this report is that of the student alone. Students were encouraged to take ownership of their projects and to develop and execute their experiments with limited guidance and assistance. The content of the research does not necessarily represent the views of the supervisor or any staff member of the University of Pretoria, Department of Civil Engineering. The supervisor did not read or edit the final report and is not responsible for any technical inaccuracies, statements or errors. The conclusions and recommendations given in the report are also not necessarily that of the supervisor, sponsors or companies involved in the research.

Elana Rautenbach

29273499

24 November 2016

Number of words: 43136 


\section{ACKNOWLEDGEMENT}

I would first like to thank my dissertation advisor Professor Ben van Rensburg of the Department of Engineering at the University of Pretoria. Whenever I ran into any difficulties or had any questions regarding my research or writing, his door was always open, and the privilege of his guidance and assistance were truly valuable resources. He allowed me the freedom to ensure this dissertation is a true reflection of myself and my work, whilst providing the gentle nudges to steer me on the path of academic excellence.

I would also like to thank the external experts I consulted with in the conceptual phase of this dissertation, Mr. Tony Aimer and Mr. John Barnard, for their input and insight during these difficult initial stages. I must also extend my gratitude to Professor Rudy Du Preez, whose continued support over the course of this dissertation proved integral to the success of my research. Without their participation and expertise, this already challenging study could not have been conducted as efficiently or successfully.

Finally, this work would not have been possible without the unconditional support of my family and friends. A special thanks to my parents, Annelie and Craigie. Finally, I want to thank the person who supported me more than anyone else - my fiancée Michael Forbes. Whenever I had questions that I did not dare ask someone else, I could ask him. Thank you so much for discussing these and many other questions with me at all possible and impossible times, for being so patient and self-sacrificing, and for always giving me strength and hope. 


\section{TABLE OF CONTENTS}

1.1 Background

1.2 Problem Statement

1.3 Study Objectives

1-8

1.4 Scope of Study

1.5 Organisation of Report

2 LITERATURE REVIEW

2.1 Background

2.1.1 Cold-formed Steel

$2-10$

2.1.2 Connections

$2-12$

2.1.3 Screw Connections

2-12

2.1.4 Self-drilling screws

2.2 Cold Formed Steel Connection Design Provisions

2.2.1 Screws in Shear

2-15

2.2.2 Net Section in Tension

$2-15$

2.2.3 Tilting and Bearing

$2-16$

2.3 Screw Connection Behaviour

$2-20$

2.3.1 Effect of Steel Sheet Thickness

$2-20$

2.3.2 Effect of Steel Ductility

$2-21$

2.3.3 Effect of Number of Screws

$2-23$

2.4 Screw Connection Strength

$2-26$

2.4.1 Effect of Number of Screws on Connection Strength

$2-26$

2.4.2 Effect of Screw Patterns on Connection Strength

$2-32$

2.5 Finite Element Analysis Of Lap Shear Connections

2.5.1 Chung \& Ip (2000; 2001)

2-35

2.5.2 Kim \& Kuwamura (2007)

2-45

2.5.3 Kim, Kuwamura \& Cho (2008)

$2-47$

2.5.4 Salih, Gardner \& Nethercot (2010)

$2-48$

2.5.5 Moze \& Beg (2011)

$2-51$

$2-54$

2.5.6 Fan, Rondal \& Cescotto (1997a; 1997b)

$2-57$

2.5.7 Explicit vs Implicit Dynamic Analysis Method

2.5.8 Yu, Burgess, Davison \& Plank (2008)

2-61

2.5.9 Lu, Ma, Makelainen \& Outinen (2012)

3 FINITE ELEMENT MODEL 3-69

$\begin{array}{llr}3.1 & \text { Introduction } & 3-69\end{array}$

$\begin{array}{ll}3.2 & \text { Modelling Considerations }\end{array}$

3.2.1 Nature of the Problem $\quad 3-70$

$\begin{array}{lll}\text { 3.2.2 Geometry and simplification of model } & \text { 3-70 }\end{array}$

3.3 Type of Element and Finite Element Mesh $\quad 3-71$

$\begin{array}{llr}3.3 .1 & \text { Elements } & 3-71\end{array}$

$\begin{array}{lll}\text { 3.3.2 Mesh 3-72 } & \text { 3-71 }\end{array}$

$\begin{array}{lll}3.4 & \text { Constraints } & \text { 3-73 }\end{array}$

3.4.1 Loading Speed and Mass Scaling $\quad 3-74$

$\begin{array}{lll}\text { 3.4.2 Loading Method } & \text { 3-74 }\end{array}$ 
3.5 Material Modelling 3-77

3.6 Interaction 3-80

3.6.1 Contact Constraint Enforcement Method 3-81

3.6.2 Contact Surface Weighting 3-82

$\begin{array}{lll}\text { 3.6.3 Energy Considerations } & 3-83\end{array}$

3.6.4 Contact Property Models 3-86

3.7 Verification of Finite Element Model 3-87

$\begin{array}{llr}3.8 & \text { Summary } & 3-90\end{array}$

$4 \quad$ PARAMETRIC STUDY 4-93

4.1 Introduction 4-93

4.2 Comparison of FEA to Failure Criterion Predicted Loads 4-94

4.2.1 Variation in the sheet steel thickness $\quad$ 4-95

4.2.2 Variation in the number of screws 4-96

4.2.3 Variation in the screw size 4-98

4.3 Effect of Sheet Steel Thickness $4-99$

4.3.1 Screw rotation 4-99

4.3.2 Stress and strain distribution $\quad 4-100$

4.4 Effect of Number of Screws $4-102$

4.4.1 Connection strength $\quad$ 4-102

4.4.2 Stress distribution 4-103

4.4.3 Screw rotation 4-106

4.4.4 Force-displacement curves 4-107

4.5 Effect of Screw Patterns $\quad$ 4-108

$\begin{array}{lll}\text { 4.5.1 Connection strength } & 4-110\end{array}$

$\begin{array}{ll}\text { 4.5.2 Screw rotation } & 4-110\end{array}$

4.6 Effect of Screw Size 4-112

4.6.1 Screw rotation $\quad$ 4-112

4.6.2 Connection strength $\quad$ 4-113

4.6.3 Stress and strain distribution 4-113

$\begin{array}{lll}4.7 & \text { Summary 4-114 }\end{array}$

5 CONCLUSIONS 5 5-116

$5.1 \quad$ Finite Element Modelling Considerations $\quad$ 5-116

5.1.1 Effect of the contact constraint enforcement method 5-116

5.1.2 Effect of the weighting between the master and slave surfaces 5-117

5.1.3 Effective control of the applied load and how the energy output was affected 5-117

5.2 Parametric Study 5-118

5.2.1 The efficacy of the FEM in predicting deflection limited based loads equated using the various design standards $\quad$ 5-118

5.2.2 Effect of sheet steel thickness in the connection $\quad 5-119$

5.2.3 Effect of the number of screws in the connection 5-119

5.2.4 Effect of the screw pattern in the connection 5-119

5.2.5 Effect of screw size in the connection 5-119

$\begin{array}{llr}5.3 & \text { Recommendations } & 5-120\end{array}$

6 REFERENCES 6-121

APPENDIX A: $\quad$ A-125

$\begin{array}{ll}\text { LIST OF DEFINITIONS } & \text { A-125 }\end{array}$

$\begin{array}{ll}\text { APPENDIX B: } & \text { B-134 }\end{array}$ 
SUPPLEMENTARY INFORMATION FOR LITERATURE REVIEW

B-134

APPENDIX C:

C-135

SUPPLEMENTARY INFORMATION FOR FINITE ELEMENT MODEL

C-135

APPENDIX D:

D-139

SUPPLEMENTARY INFORMATION FOR PARAMETRIC STUDY

D-139 


\section{LIST OF TABLES}

$\begin{array}{ll}\text { Table 2-1 Screw gauge number with corresponding screw diameter } & \text { 2-14 }\end{array}$

Table 2-2 Bearing Resistance Factor, C 2-17

Table 2-3 Comparison of maximum load capacities (per screw) for 2-screw and 4-screw $\begin{array}{lr}\text { connections } & \text { 2-28 }\end{array}$

Table 2-4 Effect of number of screws on shear strength $\quad$ 2-30

Table 2-5 Steel Mechanical Properties $\quad$ 2-31

Table 2-6 Typical Results for Four Screw Patterns $\quad$ 2-32

Table 2-7 Effect of Number of Columns on Connection Strength $\quad$ 2-33

Table 2-8 Effect of Cupping $\quad$ 2-34

Table 2-9 Coupon test results at normal room (ambient) temperature 2-65

$\begin{array}{lr}\text { Table 3-1 Material properties of thin sheet steels } & \text { 3-79 }\end{array}$

Table 3-2 Contact Property Models (Yan, 2012) 3-87

Table 4-1 FEA-to-predicted results for different sheet thicknesses 4-96

Table 4-2 FEA-to-predicted results for variation in the number of screws 4-96

Table 4-3 FEA-to-predicted results for variation in the number of screws excluding reduced net section in tension equation $\quad$ 4-97

Table 4-4 Comparison of FEA to predicted loads for variation in screw size 4-98

Table 4-5 Effect of screw pattern on screw rotation at 5 mm displacement 4-111

Table 4-6 Effect of screw size on screw rotation at 5 mm displacement $\quad$ 4-112

\section{LIST OF FIGURES}

PAGE

Figure 1-1 Steel Frame Housing (Barnard, 2011) $\quad$ 1-2

Figure 1-2 Maputo Apartments (Barnard, 2011) $1-2$

Figure 1-3 Lightweight roof truss for Zambesi Mall spanning $22.5 \quad 1-4$

Figure 1-4 Rooftop extension of an existing building at Plymouth University 1-4

Figure 1-5 Rooftop extension of existing building in Rotterdam 1-5

Figure 1-6 New light steel infill walls by Metsec for rooftop extension 1-5

$\begin{array}{lr}\text { Figure 2-1 Screw loaded in shear and tension } & \text { 2-13 }\end{array}$

$\begin{array}{lr}\text { Figure 2-2 Shearing of screws } & \text { 2-15 }\end{array}$

$\begin{array}{lr}\text { Figure 2-3 Net section tension failure } & \text { 2-16 }\end{array}$

$\begin{array}{lr}\text { Figure 2-4 Bearing Failure } & 2-18\end{array}$

$\begin{array}{lr}\text { Figure 2-5 Tilting mode of failure } & \text { 2-18 }\end{array}$

$\begin{array}{lr}\text { Figure 2-6 Tilting failure } & 2-19\end{array}$

$\begin{array}{lr}\text { Figure 2-7 Bearing/Tilting failure } & \text { 2-19 }\end{array}$

Figure 2-8 Bearing and pull-through or pull-over failure 2-20

Figure 2-9 Load versus deflection for single screws in single shear for normal ductility steel ( $\mathrm{t}$

$\left.=1.04 \mathrm{~mm}, \mathrm{f}_{\mathrm{y}}=196 \mathrm{MPa}, \mathrm{f}_{\mathrm{u}}=336 \mathrm{MPa}\right)$ and low ductility steel $\left(\mathrm{t}=1.09 \mathrm{~mm}, \mathrm{f}_{\mathrm{y}}=623 \mathrm{MPa}\right.$,

$\left.\mathrm{f}_{\mathrm{u}}=673 \mathrm{MPa}\right) \quad 2-22$

Figure 2-10 Load versus deflection for single screws in single shear for normal ductility steel $\left(\mathrm{t}=1.83 \mathrm{~mm}, \mathrm{f}_{\mathrm{y}}=403 \mathrm{MPa}, \mathrm{f}_{\mathrm{u}}=458 \mathrm{MPa}\right)$ and low ductility steel $\left(\mathrm{t}=1.73 \mathrm{~mm}, \mathrm{f}_{\mathrm{y}}=746 \mathrm{MPa}\right.$, $\left.\mathrm{f}_{\mathrm{u}}=784 \mathrm{MPa}\right) \quad 2-22$

$\begin{array}{lr}\text { Figure 2-11 Configuration for test specimens } & \text { 2-23 }\end{array}$ 
$\begin{array}{ll}\text { Figure 2-12 Failure modes for sheets of identical thickness } & \text { 2-24 }\end{array}$

Figure 2-13 Failure modes of sheets of different thickness $\quad$ 2-24

Figure 2-14 Load vs. displacement curves for T+NSF \& T+B+PO failure modes $\quad 2-25$

Figure 2-15 Failure modes of screw connections $\quad$ 2-26

Figure 2-16 Effect of number of screws on connection strength ( $\mathrm{t}=0.9 \mathrm{~mm}$, No.12 Screws) 2-

28

Figure 2-17 Group effect versus number of screws $(\mathrm{t}=1.5 \mathrm{~mm}$, No. 10 screws $) \quad 2-29$

$\begin{array}{ll}\text { Figure 2-18 Load versus number of screws } & 2-30\end{array}$

Figure 2-19 Ratio $\mathrm{P}_{\mathrm{ut}} / \mathrm{P}_{\mathrm{ut}, \text { 2-screws }}$ versus number of screws $\quad$ 2-31

Figure 2-20 Screw Patterns for Four Screws $\quad$ 2-32

Figure 2-21 Screw patterns for two screws $\quad$ 2-33

$\begin{array}{ll}\text { Figure 2-22 Screw patterns for ten and twelve screws } & 2-34\end{array}$

$\begin{array}{ll}\text { Figure 2-23 Failure Due to Cupping of the Steel } & 2-35\end{array}$

Figure 2-24 Configuration of typical test specimen in lap shear test $\quad$ 2-36

$\begin{array}{ll}\text { Figure 2-25 Overall view of connection } & 2-37\end{array}$

$\begin{array}{ll}\text { Figure 2-26 Enlarged view of bolt and washer } & 2-37\end{array}$

$\begin{array}{lr}\text { Figure 2-27 Proposed stress-strain curves } & 2-38\end{array}$

Figure 2-28 Deformed shape at $3 \mathrm{~mm}$ extension $\quad 2-40$

Figure 2-29 Stress distribution of bolted connection G550 specimen at $3 \mathrm{~mm}$ extension $\quad$ 2-40

$\begin{array}{ll}\text { Figure 2-30 Von Mises stress distribution of bolted connection } & \text { 2-41 }\end{array}$

Figure 2-31 Stress distribution of bolted connection G550 specimen $\quad$ 2-42

Figure 2-32 Strain components of bolted connection G550 specimen at 3 mm extension 2-43

Figure 2-33 Stress distribution of bolted connections at 3 mm extension $\quad 2-44$

$\begin{array}{ll}\text { Figure 2-34 Details of finite element models } & \text { 2-46 }\end{array}$

Figure 2-35 Deformed shape showing curling $\quad$ 2-46

$\begin{array}{ll}\text { Figure 2-36 FE model with boundary conditions } & \text { 2-47 }\end{array}$

Figure 2-37 Difference of curling behaviour according to plate thickness $\quad$ 2-48

$\begin{array}{ll}\text { Figure 2-38 Stress-strain curves for carbon and stainless steel } & \text { 2-49 }\end{array}$

Figure 2-39 Comparison between deformed test specimens and numerical model $\quad$ 2-50

Figure 2-40 Numerical model of connection 2-52

Figure 2-41 Comparison of numerical and experimental load-displacement curves of standard

$\begin{array}{lr}\text { tensile test } & 2-53\end{array}$

Figure 2-42 Von Mises stress plotted over the actual specimens $\quad$ 2-53

$\begin{array}{ll}\text { Figure 2-43 Screw thread simplification } & 2-54\end{array}$

Figure 2-44 Finite element mesh, boundary conditions and applied force 2-55

Figure 2-45 The mechanical properties of $0.63 \mathrm{~mm}$ steel sheet used in the numerical

$\begin{array}{ll}\text { simulation } & \text { 2-56 }\end{array}$

Figure 2-46 Comparison of test results from static analysis and explicit dynamic analysis for a

$\begin{array}{lr}\text { lap joint test } & \text { 2-60 }\end{array}$

Figure 2-47 FE model of single lap screw sheeting connection 2-62

Figure 2-48 Material model at room and elevated temperature for S350 steel 2-62

Figure 2-49 Load displacement curves and failure mode observed for screwed connections 263

Figure 2-50 Test set-up of single shear screwed connections of thin sheet steels at elevated $\begin{array}{ll}\text { temperatures } & \text { 2-64 }\end{array}$

Figure 2-51 Boundary conditions and reference nodes of FE model 2-65

Figure 2-52 FEA curves for 120-190 screwed connections at $22{ }^{\circ} \mathrm{C}$ and $600{ }^{\circ} \mathrm{C} \quad 2-66$

Figure 3-1 Mesh size in overlapped section of specimen $\quad$ 3-72 
Figure 3-2 Finite element model with boundary conditions

Figure 3-3 Example of an amplitude definition using a smooth step 3-75

Figure 3-4 Effect of loading amplitude on load displacement curve 3-76

Figure 3-5 Ratio between kinetic and internal energy for model 3-77

Figure 3-6 Mises yield surface (plan view of $\boldsymbol{\pi}$-plane) and associate strain-increment vector 378

Figure 3-7 Conflicting constraint conditions

Figure 3-8 Penetration of master nodes into slave surface with pure master-slave contact 3-83

Figure 3-9 Balanced master-slave contact constraint with kinematic compliance $\quad 3-83$

Figure 3-10 Deformation of model with kinematic contact enforcement 3-84

Figure 3-11 Deformation of model with penalty contact enforcement 3-85

Figure 3-12 Loss in total energy accounted for in contact energy due to penalty contact $\begin{array}{ll}\text { constraint } & \text { 3-85 }\end{array}$

Figure 3-13 Excessive penetrations of master surface into slave surface 3-86

Figure 3-14 Slip regions for the basic coulomb friction model 3-87

Figure 3-15 Different failure modes of crack propagation (Ugural \& Fenster, 2012) 3-88

Figure 3-16 FEA curve of reaction force vs displacement $3-88$

Figure 3-17 Side profile of deformed model with Von Mises stress plot $\quad 3-89$

Figure 3-18 Top view of deformed model with Von Mises stress plot $\quad 3-89$

Figure 3-19 View of deformed model with PEEQ plot $\quad 3-90$

Figure 4-1 Definition of P3. O - FEA, P6. 35 - FEA and Pultimate 4-94

Figure 4-2 Reference nodes at screw holes for estimation of displacement of connection 4-95

Figure 4-3 Bearing/Tilting failure for specimen 120-190-S4-P-14 at maximum deflection based load $\quad$ 4-97

Figure 4-4 Net section fracture failure for specimen 120-190-S4-P-14 at 5 mm extension 4-97

Figure 4-5 Decrease in screw rotation as bottom sheet thickness increases for specimens 4-99

Figure 4-6 Decrease in screw rotation as top sheet thickness decreases for specimens 4-99

Figure 4-7 Effect of sheet steel thickness of screw rotation 4-100

Figure 4-8 Top sheet with von Mises stress plots at $3 \mathrm{~mm}$ extension for specimens $\quad$ 4-101

Figure 4-9 Effect of sheet steel thickness on plate strains 4-101

Figure 4-10 Relationship between connection strength and number of screws 4-102

Figure 4-11 Group effect of connections against number of screws in connection 4-103

Figure 4-12 Top sheet with von Mises stress plot for specimen 120-190-S3-P-14 4-104

Figure 4-13 Top view of von Mises stress plot for specimen 120-190-S5-P-14 4-104

Figure 4-14 Top view of von Mises stress plot for specimen 120-190-S5-P-14 4-105

Figure 4-15 Top view with PEEQ plot for specimen 120-190-S5-P-14 4-105

Figure 4-16 Numbering of screws arranged parallel to the applied load in connections 4-106

Figure 4-17 Effect of number of screws in connection on screw rotation 4-107

Figure 4-18 Force-displacement curves for increase in the number of screws in the connection

4-108

Figure 4-19 Screw connection patterns $\quad$ 4-109

Figure 4-20 Force-displacement curves for various screw patterns 4-110

Figure 4-21 Effect of screw patterns on screw rotation for two screw connection specimens 4111

Figure 4-22 Effect of screw patterns on screw rotation for four screw connection specimens 4111

Figure 4-23 Overlay plot showing decrease in rotation with increase in screw size for specimens 
Figure 4-24 Effect of screw size on connection strength at $5 \mathrm{~mm}$ displacement

Figure A-1 Shear bands formed during compression of a consolidated cylinder of nanocrystalline Fe (grain size $80 \mathrm{~nm}$ ) (Greer et al., 2013)

Figure A-2 Von Mises yield criteria for plane stress showing yield ellipse (Chen \& Han, 1998)

Figure A-3 Master surface allowed to penetrate slave surface (ABAQUS, 2015)

Figure A-4 Shape change of the material block under the moment in the ideal situation A-129

Figure A-5 Shape change of the fully integrated first order element under moment

Figure A-6 Shape change of the fully integrated second order element under moment A-130

Figure A-7 Shape change of the reduced integration element under moment

Figure B-8 Screwed connection specimens - Nominal Dimensions

B-134

Figure B-9 Screw head descriptions (Serrette \& López, 1996)

B-134

Figure C-10 Example of (a) elastic material property; and (b) plastic material property input

Figure C-11 Mass Scale Factor Input

Figure C-12 Contact surface interaction definition example

Figure C-13 Contact property definition for (a) tangential behaviour; and (b) normal behaviour example

Figure C-14 Kinematic Coupling Constraint Definitions for (a) RP1 - Top Plate Edge; and (b) RP2 - Bottom Plate Edge

Figure C-15 Load Amplitude Definition

Figure C-16 Boundary Conditions as defined for the two reference points (a) Load definition for edge of top plate; (b) Restraints for edge of top plate allowing movement only in direction of loading; and (c) restraints for edge of bottom plate which is fixed against movement in all directions

Figure D-17 Force-displacement curves for variation in sheet steel thickness with a bottom plate thickness of $0.80 \mathrm{~mm}$

Figure D-18 Force-displacement curves for variation in sheet steel thickness with a bottom plate thickness of $1.00 \mathrm{~mm}$

D-139

Figure D-19 Force-displacement curves for variation in sheet steel thickness with a bottom plate thickness of $1.20 \mathrm{~mm}$

D-140

Figure D-20 Force-displacement curves for variation in screw size

D-140 


\section{LIST OF NOTAION}

All symbols are defined where they first appear in the text. In most cases only one meaning has been assigned to each symbol, but where this is not the case, the interpretation will be evident from the text. The symbols are listed as follows:

\begin{tabular}{|c|c|}
\hline Symbol & Description \\
\hline$\overline{A_{n}}$ & Net area of the connected part in the cross section being analysed \\
\hline$A_{0}$ & Net area of the section prior to deformation \\
\hline$[\mathrm{C}]$ & Global damping matrix \\
\hline C & Tilting/bearing coefficient \\
\hline$c_{d}$ & Dilatational wave speed of the material \\
\hline$d$ & Nominal screw diameter \\
\hline$E$ & Initial Young's Modulus \\
\hline$E_{C W}$ & Work done by the constraint penalties \\
\hline$E_{F D}$ & Frictional energy dissipated \\
\hline$E_{H F}$ & External heat energy through external fluxes \\
\hline$E_{I}$ & Internal energy \\
\hline$E_{I H E}$ & Internal heat energy \\
\hline$E_{K E}$ & Kinetic energy \\
\hline$E_{M W}$ & Work done by the propelling added mass \\
\hline$E_{P W}$ & Work done by the contact penalties \\
\hline$E_{\text {TOTAL }}$ & Total energy which is equated by the summation of all the energies \\
\hline$E_{V}$ & Viscous energy dissipated \\
\hline$E_{W}$ & Work done by externally applied loads \\
\hline$E_{0.2}$ & Tangent stiffness at the $0.2 \%$ proof stress \\
\hline$F$ & Force applied to the section being analysed \\
\hline$\{\mathrm{F}(\mathrm{t})\}$ & Time dependant load vector \\
\hline$F_{y}$ & Yield strength of the sheet being analysed \\
\hline$F_{u}$ & Tensile strength of the sheet steel being analysed \\
\hline$F_{u 1}$ & Tensile strength of the top sheet steel in the connection \\
\hline$F_{u 2}$ & Tensile strength of the bottom sheet steel in the connection \\
\hline
\end{tabular}




\begin{tabular}{|c|c|}
\hline$f^{2}$ & Mass scaling factor \\
\hline$f_{0.2}$ & $0.2 \%$ Proof stress \\
\hline I & Internal Energy \\
\hline K & Strength constant \\
\hline$[\mathrm{K}]$ & Global stiffness matrix \\
\hline$L^{e}$ & Characteristic element length \\
\hline$l_{0}$ & Length of the section prior to deformation \\
\hline $\mathrm{LE}_{\text {top }}$ & Strain in the top plate of the connection \\
\hline $\mathrm{LE}_{\text {bot }}$ & Strain in the bottom plate of the connection \\
\hline$[\mathrm{M}]$ & Global mass matrix \\
\hline$m$ & Strain hardening coefficient for the Romberg-Osgood model \\
\hline$n$ & Strain hardening coefficient for the Romberg-Osgood model \\
\hline$p$ & Strain hardening exponent \\
\hline$P$ & Mean shear strength of the connection \\
\hline PEEQ & Equivalent plastic strain \\
\hline$P_{n}$ & Nominal tensile strength of the net section \\
\hline$P_{n s}$ & Nominal connection shear strength \\
\hline$P_{S S}$ & Nominal shear strength of a screw \\
\hline Pultimate & Maximum load attainable by the connection \\
\hline py & Measured yield strength of the material \\
\hline$P_{1}$ & Shear strength of a single screw connection \\
\hline$P_{3.0-F E A}$ & $\begin{array}{l}\text { Maximum load, as determined by the FEM, at/before a screw hole } \\
\text { displacement of } 3 \mathrm{~mm}\end{array}$ \\
\hline$P_{6.35-F E A}$ & $\begin{array}{l}\text { Maximum load, as determined by the FEM, at/before a screw hole } \\
\text { displacement of } 6.35 \mathrm{~mm}\end{array}$ \\
\hline$s$ & Width of the sheet being considered in the connection \\
\hline SRSS & Square root of the sum of squares \\
\hline$t_{1}$ & Thickness of the top sheet steel in the connection \\
\hline$t_{2}$ & Thickness of the bottom sheet steel in the connection \\
\hline$t$ & Thickness of connected part being analysed \\
\hline$\{U\}$ & Nodal displacement vector \\
\hline
\end{tabular}


$\mathrm{U}_{\mathrm{s}}$

$U^{n+1}$

$u_{1}$

$u_{2}$

$u_{3}$

$\mathrm{V}_{\mathrm{p}}$

$\alpha$

$\Delta \mathrm{t}$

$\Delta l$

$\varepsilon$

$\varepsilon_{\mathrm{x}}$

$\varepsilon_{\text {true }}^{p l}$

$\varepsilon_{0.2}$

$\varepsilon_{u}$

$\varepsilon_{f}$

$\varepsilon_{n}$

$\bar{\varepsilon}^{p l}$

$\dot{\varepsilon}^{p l}$

$\Psi$

$\sigma$

$\sigma_{\text {frac,true }}$

$\sigma_{n}$

$\sigma_{\text {true }}$

$\sigma_{u, t r u e}$

$\sigma_{11}$

$\sigma_{22}$

$\sigma_{33}$

$\sigma_{0.2}$

$\tau_{1}$

$\tau_{2}$
Bi-linear stress-strain curve using measured tensile stress

Current nodal displacement

Translational degree of freedom in local axis direction 1

Translational degree of freedom in local axis direction 2

Translational degree of freedom in local axis direction 3

Dilatational wave velocity

Tilting/bearing coefficient

Minimum stable time increment

Change in length of the material

Nominal strain of the material

Maximum direct strain

True plastic strain of the material

Total strain at the $0.2 \%$ proof stress

Strain at ultimate stress

Maximum strain after fracture

Nominal strain of the material

Equivalent plastic strain

Equivalent plastic strain rate

Axial modulus of the material

Nominal stress of the material

True fracture stress of the material

Nominal stress of the material

True stress of the material

True ultimate stress of the material

Direct stress in local direction 11

Direct stress in local direction 22

Direct stress in local direction 33

$0.2 \%$ Proof stress

Orthogonal component of the shear stress acting in local direction 1

Orthogonal component of the shear stress acting in local direction 2 
$\tau_{12}$

$\tau_{23}$

$\tau_{13}$

$\mu$

Shear stress in local direction 12

Shear stress in local direction 23

Shear stress in local direction 13

Coefficient of friction 


\section{INTRODUCTION}

\subsection{BACKGROUND}

Structural steel is generally divided into hot-rolled sections and cold-formed sections. These two types of steel sections can be distinguished by the method utilised in their formation and manufacture. Hot-rolled steel sections are created under high temperatures, up to $1400{ }^{\circ} \mathrm{C}$, in either a blast furnace or an electric arc furnace, whilst cold-formed steel sections are manufactured at room temperature (Lee et al., 2014). Cold-formed steel, which is also referred to as light gauge steel, is assimilated from steel plate, sheet or strip material in rollforming machines, or by press brake or bending brake methods. The differences in the manufacturing of these materials thus accounts for the disparities between the material properties of each, both in terms of their strength and structural performance, as well as their final mode of failure (Yu, 2000). The structural behaviour of the members in bending or compression depends on the material thickness and, to a greater extent, the shape of the section. Due to the comparative thinness of these members, their slenderness means that control of local and member buckling, local web crushing and connection design are important structural considerations.

Traditionally, steel structures would be composed of hot-rolled steel members, which possess relatively high plate thicknesses, and correspondingly, weight. Resultantly, in recent years, the use of cold-formed sections has become an increasingly popular choice for applications where heavier hot-rolled members are deemed ill-suited. The use of cold-formed structural steel members has been of growing importance in the field of lightweight steel construction due to various advantages such as: high strength-to-weight ratio, ease of transportation and construction, mass production and expedient installation. The use of light steel framing has become a popular construction choice in low to medium rise building and residential construction because it provides numerous advantages as compared to traditional construction methods.

Light-steel frame building (LSFB) is a contemporary building method which is in widespread use in Australia, the USA and Europe, and has been for well over half a century (Barnard, 2011). Initially, the LSFB industry was limited to single- and double-storey residential buildings (Figure 1-1); however, the advantages afforded by the building methodology, including the speed of construction, low building mass and good insulation properties, lend themselves excellently to other types of buildings as well. Subsequently, LSFB has been increasingly utilised for internal and external walling in multi-storey commercial and industrial buildings (Figure 1-2). 


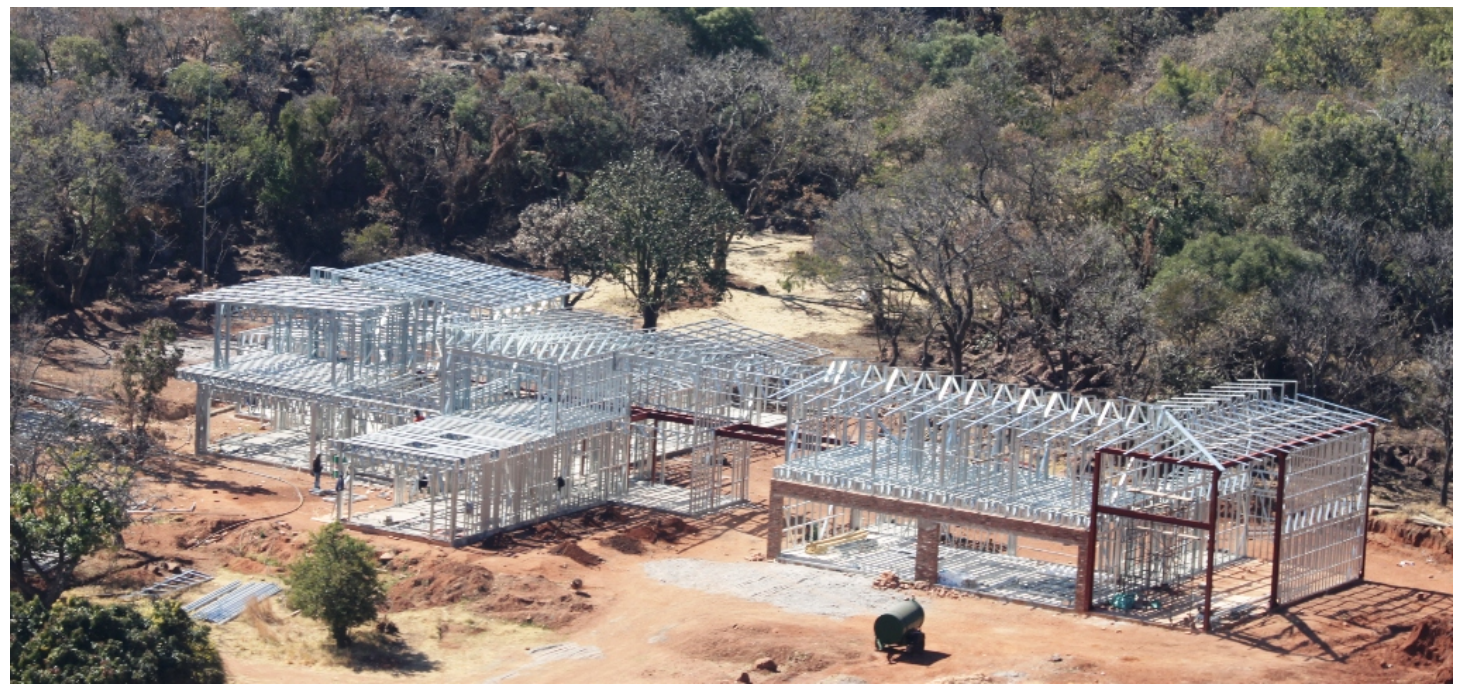

Figure 1-1 Steel Frame Housing (Barnard, 2011)

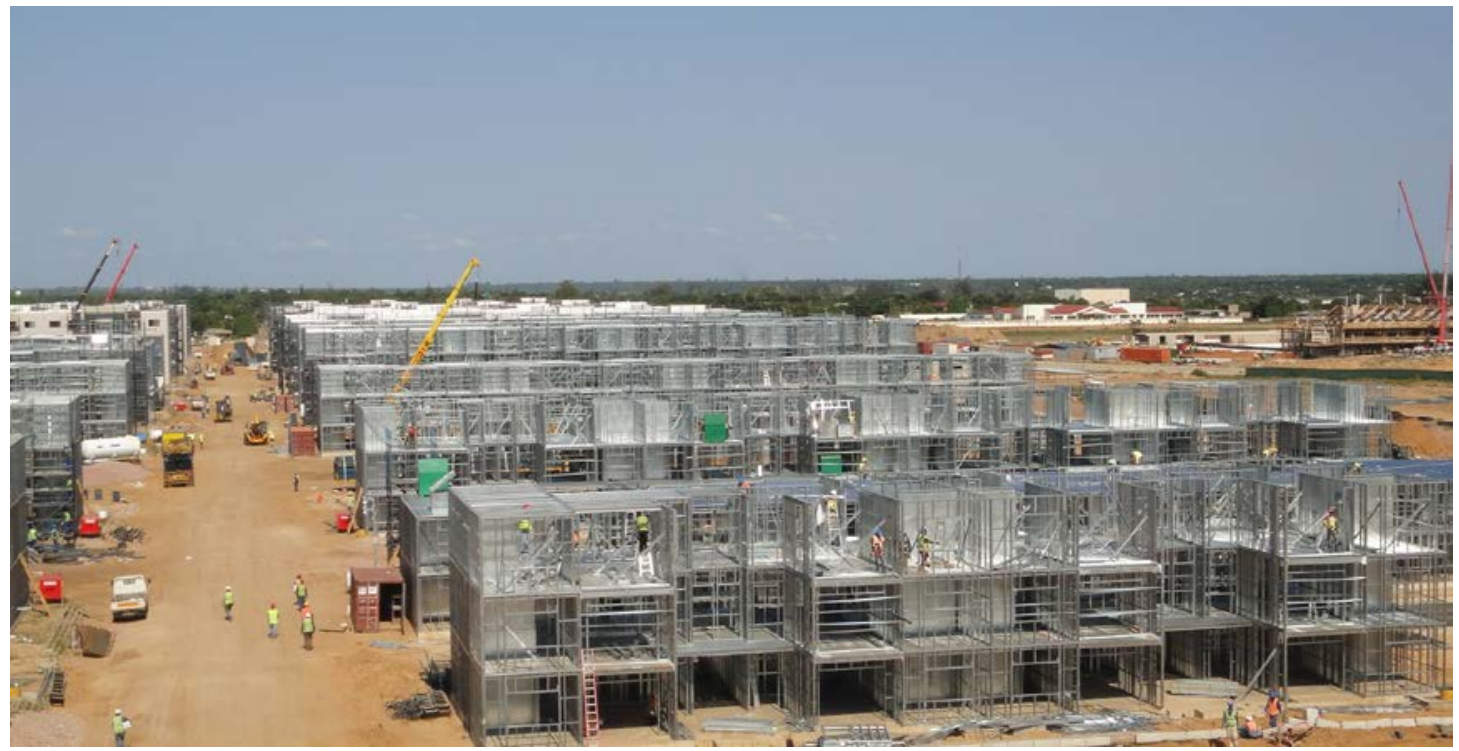

Figure 1-2 Maputo Apartments (Barnard, 2011)

The low mass of LSFB walling, less than $10 \%$ that of a conventional masonry wall, thus reduces the load on the slabs of multi-storey buildings, enabling a reduction in not only the thickness of the slab itself, but also the columns supporting the reduced load. Lighter slabs and slimmer columns could then further reduce the load on the building foundation, with the final knock on effect resulting in a mass and cost saving across the entire project.

Renovation has increasingly come to the fore as an important social and economic requirement of the building industry. Whilst adapting the building for its new intended use, it is pragmatic to retain as much of the initial structure as possible, in order to cut costs and improve efficiency, which encourages an innovative and multidisciplinary approach. The different forms of renovation include: 
- The repurposing of an existing building for another use or for occupancy;

- The addition of pitched roofs, in order to provide additional lodging and/or to address water penetration issues;

- The alteration of the building's façade through the addition of windows, overcladding and/or other facilities, as well as to decrease external heat loss;

- The addition of new amenities to a building, such as toilets, bathrooms, lifts, stairs, etc.;

- The reinforcement or rehabilitation of the existing building so as to increase its loadbearing capacity or extend the serviceability lifetime.

LFSB has carved a niche for itself in the renovation sector, especially in applications relating to the extension and/or adaptation of existing buildings. Against this backdrop of renovation, the primary applications for LSFB and other steel section components are:

- New internal separating walls and partitions, which must be lightweight in addition to possessing good acoustic and fire-resistant properties;

- Additional infill walls supporting new cladding, or alternatively, over-cladding of pre-existing façades utilising light steel sub-frames, which are capable of supporting a wide range of light-weight cladding materials;

- Refurbishing of existing flat roofs, which often has the added effect of creating additional habitable spaces;

- New modular units used to extend buildings, containing toilet or bathroom units, or external lifts and stairs;

- New mezzanine floors in buildings which have suitable floor-to-ceiling height ratios.

A few examples highlighting these various different potential applications are presented in Figure 1-3 to Figure 1-6. LSFB is used in the following forms for the purpose of "overroofing":

- Closely or widely spaced trusses spanning between façade walls, with supporting purlins between widely spaced trusses;

- Moment-resisting structures, such as portal frames, supported on the perimeter columns;

- A grillage of steel beams supporting a light-weight steel structure. 


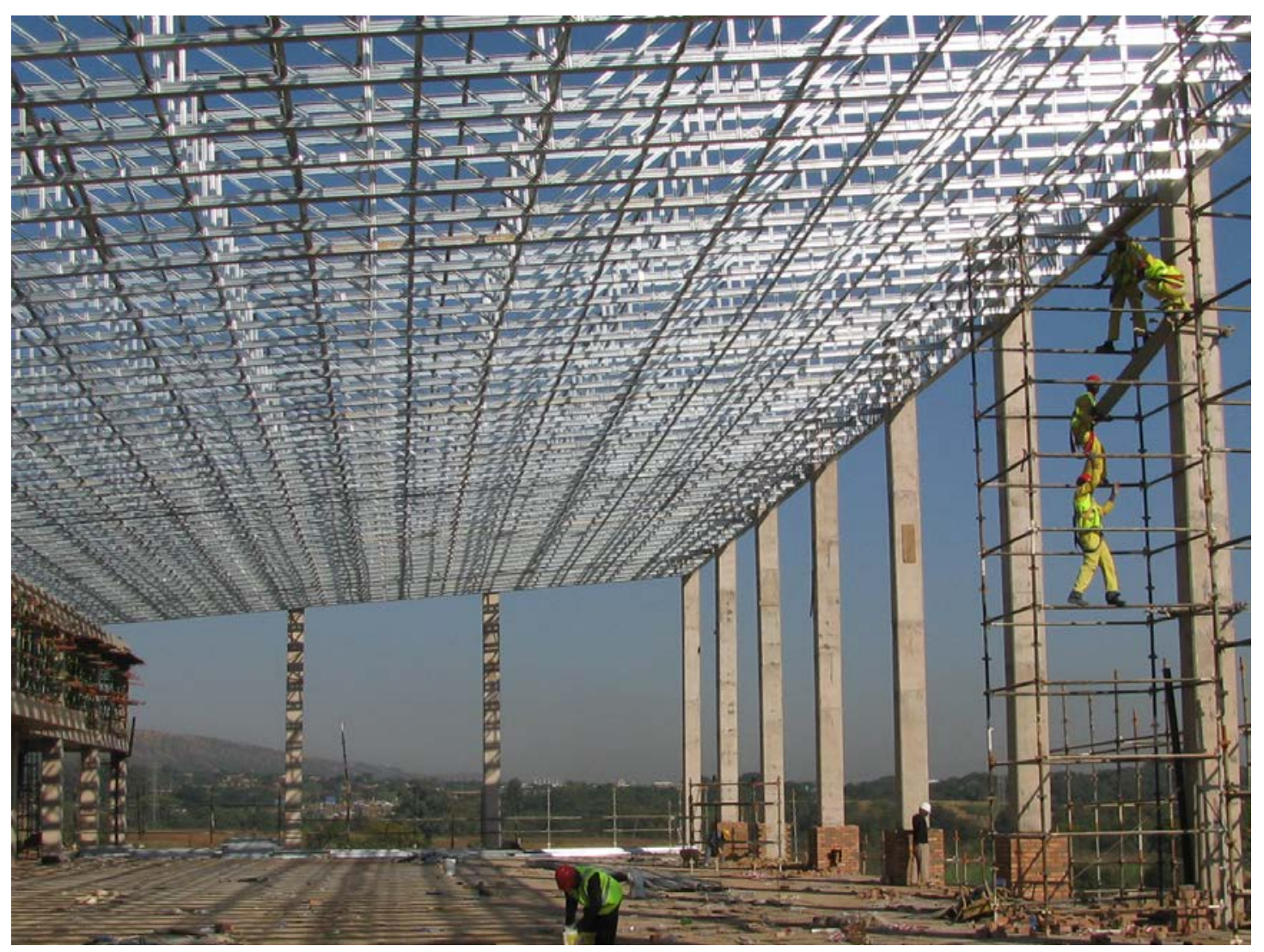

Figure 1-3 Lightweight roof truss for Zambesi Mall spanning 22.5

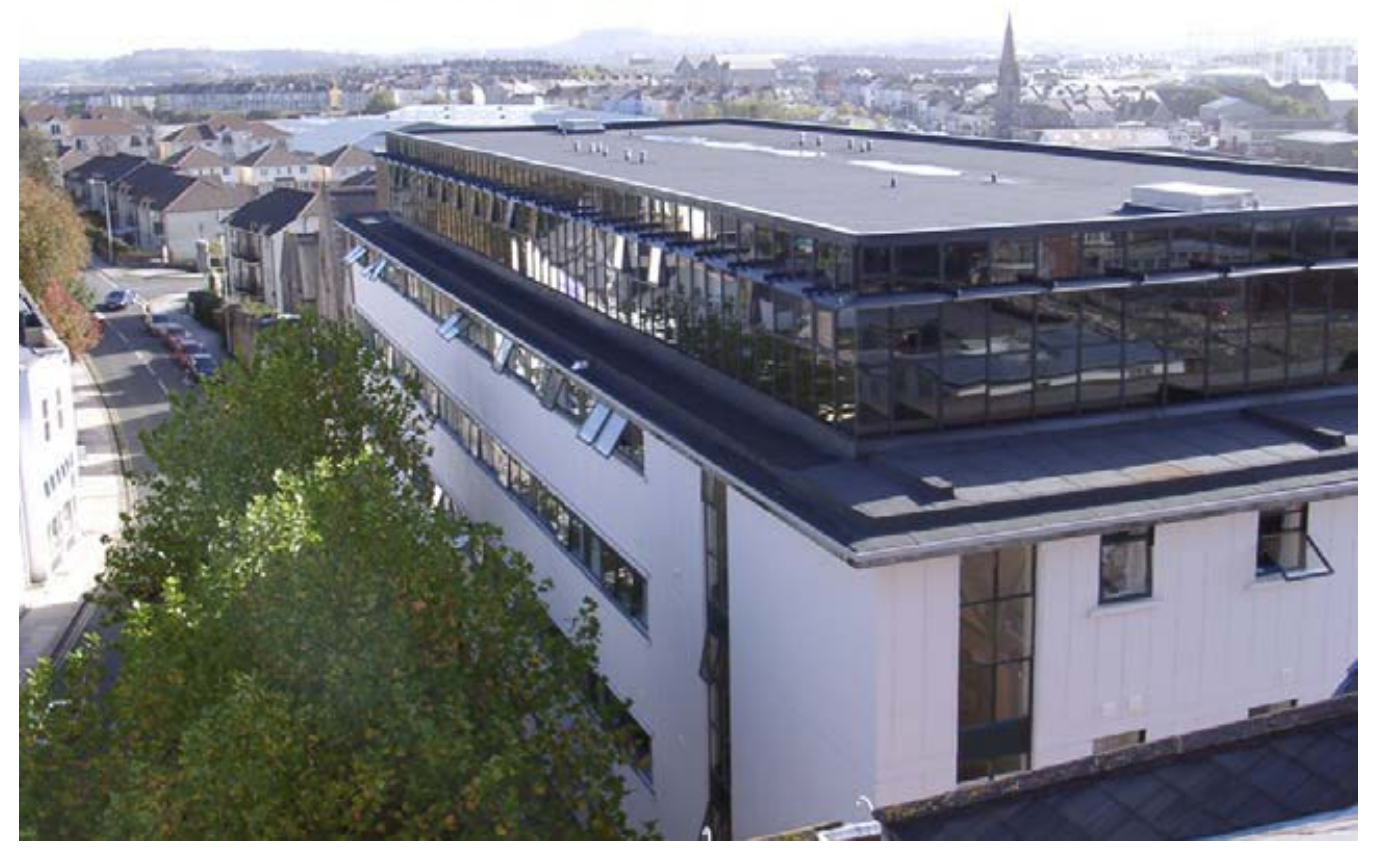

Figure 1-4 Rooftop extension of an existing building at Plymouth University 


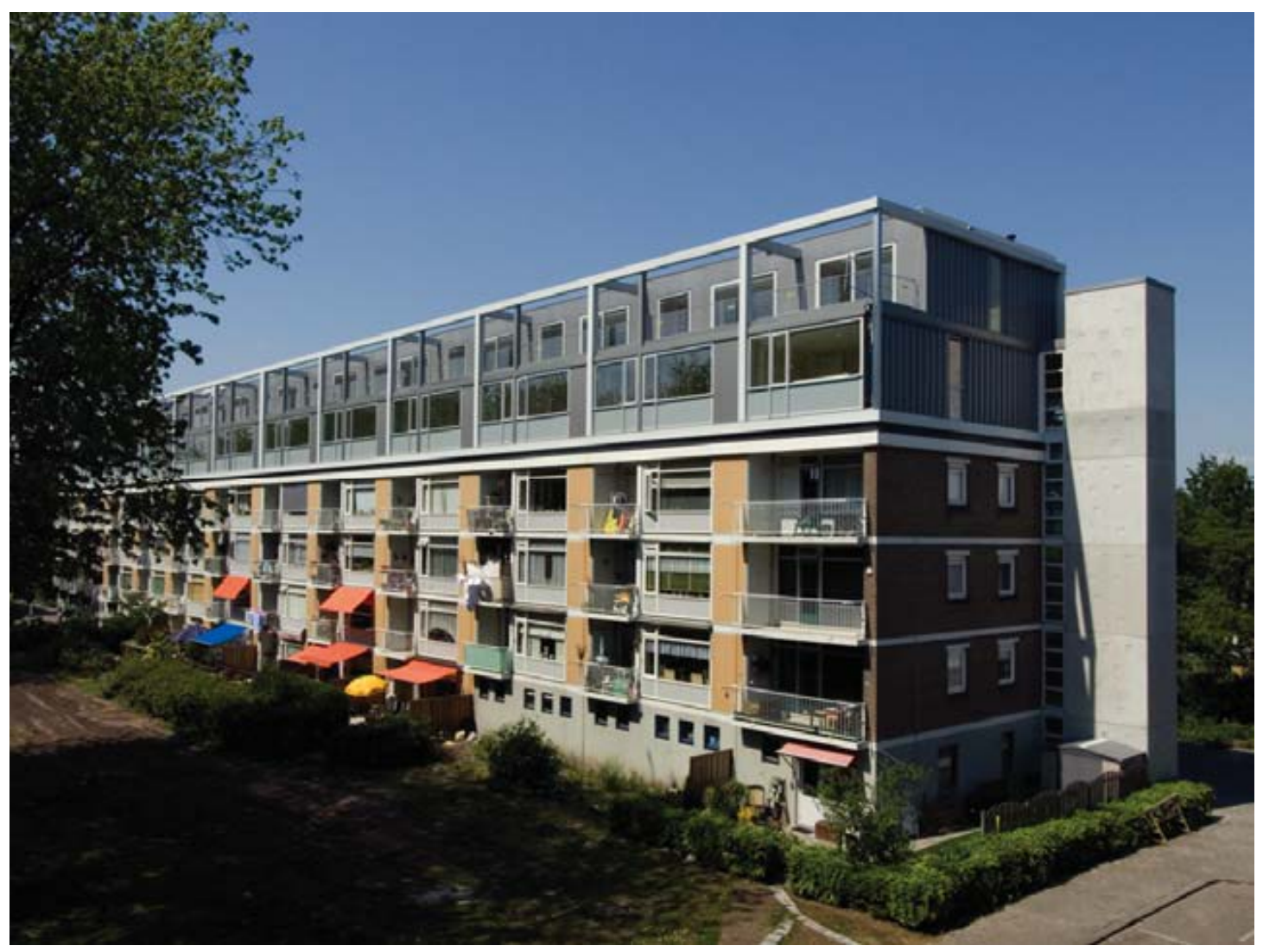

Figure 1-5 Rooftop extension of existing building in Rotterdam

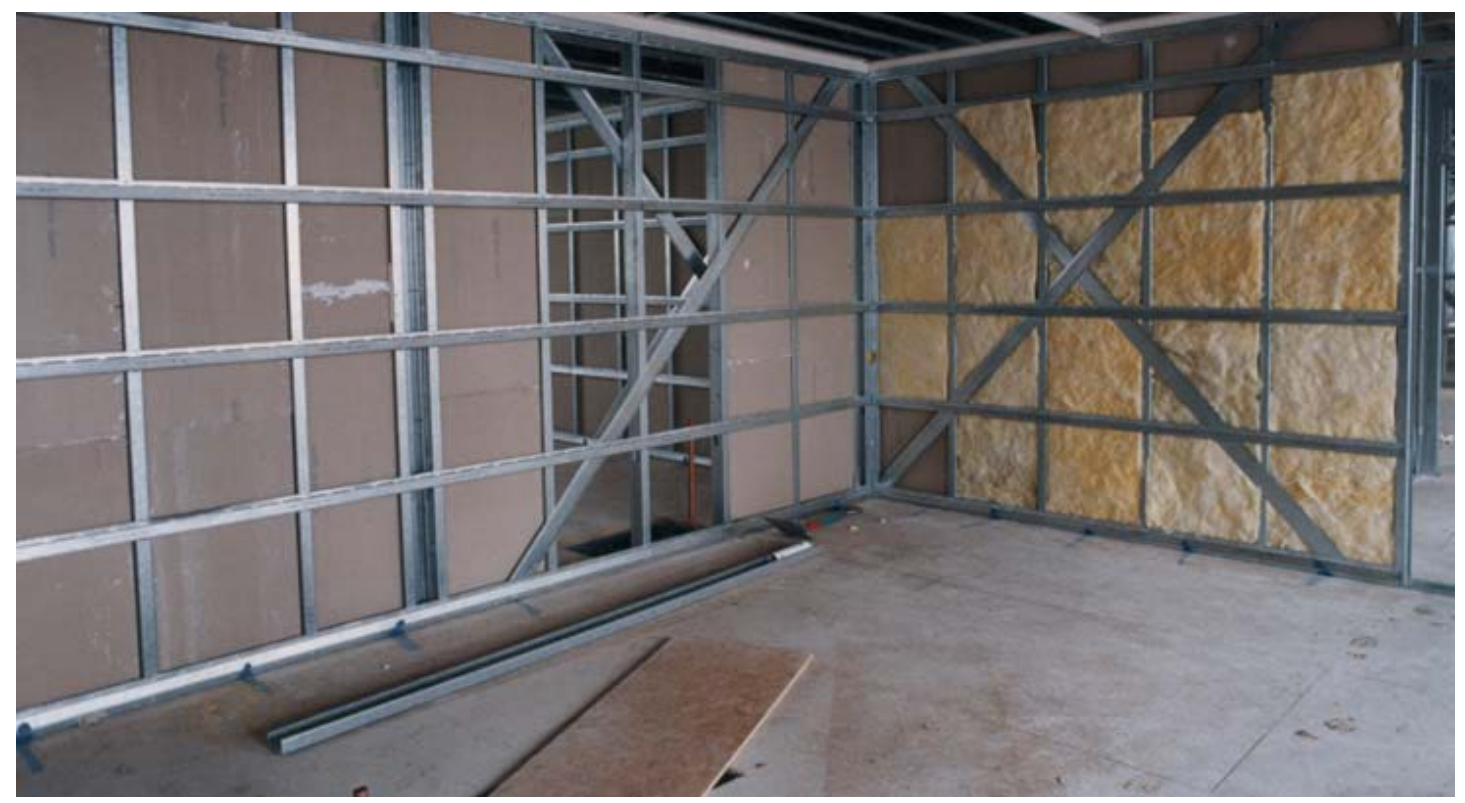

Figure 1-6 New light steel infill walls by Metsec for rooftop extension 
Joints are an important component of every structure, not only from the aspect of the structural behaviour, but also in relation to the production costs. It has been noted that for a structure consisting of hot-rolled sections, approximately $30 \%$ of the total costs are directly or indirectly influenced by the connections (Toma \& Stark, 1978). Whilst there is no data suggesting that this cost item will be much lower for light-weight structures, and the increased complexity of joining light-weight items might increase the cost thereof, there are a variety of joining methods available for such structures. The selection of the correct method is governed by a variety of factors, but the structural behaviour of the specific connection will be the most influential.

Structural systems transfer their loads through a series of elements by means of connections. Each connection is designed so that it can transfer, or support, a specific type of load or loading condition. The fabrication of connections is the most labour-intensive aspect of the construction process in cold-formed steel structures, hence a better understanding of the behaviour of these connections could reduce fabrication costs, leading to an optimal design. The fasteners utilised in hot-rolled steel connections have been specifically developed for use in thicker plates, which means that whilst these fasteners can still be used for thinner plates, there will on occasion be differences in their structural behaviour. As a result, special fasteners have been developed, and are frequently preferred for their simplicity of assembly, or due to economic factors.

\subsection{PROBLEM STATEMENT}

The behaviour of screw connections and bolt connections associated with the thin-walled steel members is distinctly atypical primarily because of the flexibility of the plates. Tilting of the fasteners in the screw hole, pullover of the screws, distortion of the sheet metal around the hole in tension, and so forth, contribute to this distinction as well. Unlike joints for hot-rolled steel structures, design guidelines for cold-formed steel connections are limited to their initial load-deflection behaviour. Detailed design procedures for cold-formed steel joint profiles are uncommon due to their wide variance and specific purpose, and most designs are based on testing results.

During the preliminary investigations for this study, several connection models were created and physically tested, in addition to the literature study, which mined existing works in order to find information pertaining to the testing and analysis of bolted and screwed thin sheet steel connections. The initial data obtained from the first experimental testing were found to correspond closely to that in the literature, which furthermore contained exhaustive physical testing of the types of samples which are contained and analysed in this study. Considering that many investigations have been conducted, and that most of those studies were more concerned about the experimental testing of steel connections, this study focussed on the finite element modelling of thin sheet steel screwed connections. 
Examination of the literature regarding the finite element analysis showed that each researcher utilised a different model, and none truly explained the rationale thereof or how the final design of the model was created. The only true correlation that could be drawn between the different researchers in terms of their finite element models, is that the process of creating an accurate model was found to be onerous and complicated. Therefore, the aim of this study was to explain and simplify the finite element modelling procedure with regards to thin sheet steel connections, that such a standardised model would be easily reproduced, in order to be able to test a variety of different connections, and be able to vary the parameters of such a model in such a way which would with reasonable accuracy represent the response of a physical experimental model.

Considering the exponential increases in computers' processing power and data handling abilities, combined with the rapid development of engineering software, numerical simulation is becoming ubiquitous in almost all fields of research. Numerical analysis has become an incredibly powerful tool in the field of structural engineering, and together with experimental analysis can provide insight into factors such as stresses and strains, which are not easily determined from experimental testing alone. Once numerical simulation has been calibrated against physical experimental tests, a large number of similar simulations can be performed with different parameters, eschewing the requirement for further physical testing, providing a highly economical solution to a complex problem.

It has been well documented that the computer modelling of screwed or bolted connections is an intricate and complicated task. Attention must be paid to: the significant geometrical and material non-linearities; contact between the components; along with the possibility of localised fracture in the vicinity of the screw, at loading levels approaching the ultimate resistance of the connection.

When compared to the body of work attributed to bolted connections, limited research reports exist regarding the numerical investigation of screwed connections. Yan (2012) attributed this to the difficulty in attaining convergence when the steel plate is less than $1 \mathrm{~mm}$ thick, combined with the complications of modelling the screw shank threading, and the uncertainty of the contact settings between the screws and plates.

It is interesting to note that the steel plates of the connections modelled in previous research, in either bolted or screwed connections, are mostly thicker than $1.20 \mathrm{~mm}$. Sheet steels which are thinner than $1.20 \mathrm{~mm}$, such as $0.8 \mathrm{~mm}$ and $0.42 \mathrm{~mm}$, are becoming an increasingly popular choice for the fabrication of members and roof sheet structures. Numerical investigation on bolted and screwed connection assemblies, with sheet steels less than 1.20 $\mathrm{mm}$ in thickness, is limited. This study is thus focussed on the finite element modelling procedure of such thin sheet steel screw connections, and how they are affected by the various modelling parameters.

The explicit solution method is a true dynamic procedure, and was originally developed in order to model high-speed impact events, where inertia plays a dominant role in the solution. When solving for a state of dynamic equilibrium, out-of-balance forces propagate as stress waves between neighbouring elements. As the minimum stable time increments are usually fairly small, most problems require a large number of increments. 
The explicit solution method has also proven valuable in the solving of static problems. The major advantage of the explicit procedure, when compared to the implicit solution method, is the ease with which it resolves complicated contact problems. However, application of the explicit dynamic procedure to quasi-static problems requires several additional special considerations such as: the types of elements used and the finite element mesh, the constraints, the material modelling and the interactions between the elements. By definition, a static solution is a long-time solution, and the analysis of the simulation in its natural time scale is often computationally impractical, often requiring an excessive number of small time increments. In order to obtain an economical solution, the event must be accelerated in some form or another. It should be noted that as the event is accelerated, the state of static equilibrium evolves into a state of dynamic equilibrium, as the inertial forces become more dominant. Therefore, the objective is to model the process in the shortest possible time, so that the inertial forces remain insignificant.

\subsection{STUDY OBJECTIVES}

The objective of the study was to investigate the finite element modelling considerations for thin sheet steel screw connections. The study was aimed at explaining the modelling technique, whereby the finite element model was calibrated with test data found in existing literature. The calibrated model was then utilised in subsequent parametric studies.

With regards to the finite element modelling procedure, the objectives included:

- To evaluate the effect of the contact conditions (kinematic vs. penalty contact tracking);

- To determine the effect of weighting between the master and slave surfaces in the contact formulation; and

- The effective control of the applied load and how the energy output is affected.

With regards to the parametric studies, the objectives included:

- The effect of sheet steel thickness as well as the number, pattern and size of the screws on connection behaviours.

- To determine the effectiveness of the finite element model in the simulation of the predicted serviceability (deflection limited) based loads, equated through the use of the various international design standards.

\subsection{SCOPE OF STUDY}

This study was focussed on the finite element modelling of thin sheet steel screwed connections testing in single shear. The finite element modelling procedure is discussed in detail. For the calibration of the finite element model, existing literature was used and thus the finite element model was calibrated to results presented by Yan (2012). In the analysis, the explicit dynamic analysis method was utilised and the model was pulled to a maximum displacement of $5 \mathrm{~mm}$ by means of the displacement controlled loading method. 
In the design calculations, the connection strength is calculated by considering all different failure modes and the lowest connection strength is taken as the design load. Since the analysis was kept to a maximum displacement of $5 \mathrm{~mm}$, a deflection based load was used to compare the results obtained through finite element analysis to that predicted by design equations. As specified in ECCS Recommendations (1987; 2009), the deflection based load can be classified as the maximum load at/or before a connection displacement of $3 \mathrm{~mm}$. In this study, it can be defined as the maximum load, as simulated by the finite element model, for the connection displacement at/before $3 \mathrm{~mm}\left(P_{3.0-F E A}\right)$. The screw hole deformation was used to determine the connection displacement.

For the parametric study, the effect of variation in various geometric properties were considered which included the effect of variation in the sheet steel thicknesses along with the variation in the size, number and pattern of the screws in the connections. The material properties were assumed to remain constant in the investigation into the effect of variation in the sheet steel thicknesses.

\subsection{ORGANISATION OF REPORT}

This dissertation consists of five chapters. The research background, statement of the problem and objectives along with scope of the study are stated in Chapter 1 . The structure of this thesis is also outlined in this chapter.

Chapter 2 gives an introduction on cold-formed steel structures and screwed connections of thin sheet steels. The design equations for screwed connections of light gauge steels are also included in this chapter. Furthermore, this chapter focusses on the literature review of experimental and numerical studies on screw connections.

In Chapter 3, the numerical investigation for the development of the finite element modelling of thin sheet steel screw connections is given using the finite element analysis program ABAQUS. The modelling considerations, constraints, material properties, contact definitions as well as the boundary conditions used in the explicit dynamic analysis are presented, all leading up to the verified finite element model.

In Chapter 4 the parametric study is conducted whereby the design equations for tilting and bearing strengths of single shear screwed connections of thin sheet steels are compared to results obtained from the finite element analysis. The effect of the various parameters considered in the parametric study are also discussed.

Chapter 5 is the last part of this dissertation in which the conclusions are summarised based on the aforementioned chapters. Recommendations for future research are also provided.

The references are listed in Chapter 6 and a list of definitions is provided in Appendix A with supplementary information for the Literature Review, Finite Element Model and Parametric Study are given in Appendix B, C and D, respectively. 


\section{LITERATURE REVIEW}

The contents of this chapter provide an overview of previous research on the use of selfdrilling screws in connections for cold-formed steel structures. A general background on coldformed steel and its' various uses will be presented, along with the different design provisions required to numerically analyse the connections in terms of their different failure modes. Numerous studies have investigated the behaviour of these connections, as well as the myriad factors which affect their ultimate capacity.

The aspects presented in this chapter, which shall be discussed in detail, include the number and spacing of the screws, in addition to various screw configurations, along with the effect of changing the thickness of the steel sheets. The different design provisions for cold-formed steel connections will be presented, along with the expected failure modes for such connections.

As the primary focus of this study regards the finite element modelling of thin sheet steel screwed connections, several different finite element models will be presented at the end of this chapter. The various parameters considered in each specific finite element model, will be discussed alongside the results obtained by the respective authors during the course of their research.

\subsection{BACKGROUND}

\subsubsection{Cold-formed Steel}

Light-gauge construction utilising galvanised cold-formed steel sections has experienced a renaissance in recent years, where previously it was used only as an adjunct to more conventional methods of construction. An area of particularly intense utilisation is in conventional, primarily industrial, steel structures as secondary and tertiary load-bearing elements, such as purlins and sheeting, on a steel or reinforced concrete building.

The structural performance of cold-formed steel sections are limited by thin-walled behaviour, resulting in premature buckling and instability. However, over the past two decades, the increased application of cold-formed steel in the construction industry has resulted in great strides being made in the research of such structural elements, in order to ensure the stability and reliability of such structures.

As stated by Davies (2000), novel developments in the practical utilisation of cold-formed steel sections in building construction, have occurred on three related platforms. Recent developments in the technology have resulted in more complex shapes, with higher yield stresses, allowing cold-formed sections to evolve into a particularly high-tech form of constructional steelwork. However, such developments would be of little consequence without the accompanying investigations into their practical application, a fact illustrated by the progressive increase in the market share of such cold-formed sections. Consequently, additional research is required into the design procedures of such structures, along with the development of their design codes and calculation models. 
As a state of the art product, cold-formed steel has much more potential for future research when compared to its more mundane hot-rolled counterpart. Several of these contemporary developments have been discussed by Davies (2000), a concise summary of which is provided below.

\section{Trend to higher quality steels}

In recent years there has been a trend towards using higher quality steels, boasting increased yield stress, in typically mass-produced products such as purlins, sheeting and decking. The steels commonly utilised in such applications currently exhibit a yield stress in the range of 280 to $550 \mathrm{~N} / \mathrm{mm}^{2}$.

\section{Trend to more complex section shapes}

Inevitably, the use of higher strength steels is accompanied by a reduction in thickness, whilst the local stability requirements necessitate the development of highly stiffened sections, comprising more folds and rolled in stiffeners. Consequently, this causes the design requirements to become more exacting as well.

\section{Improved corrosion resistance}

One of the principal motivations for the expansion of the practical utilisation of cold-formed sections is undoubtedly its exceptional corrosion resistance. This is due to the advances in galvanisation and other coating technologies, whilst taking cognisance of the fact that these coating systems are undamaged by the cold-forming process.

\section{Improved rolling and forming technology}

State of the art rolling lines are computer controlled directly by the design office, allowing highly accurate, complex shapes to be custom made on demand; replete with holes, perforations and slots, such as web openings for services, which are precisely punched during the rolling process.

\section{Improved connection technology}

Considering the thinness of cold-formed steels, the connection mechanics play a vital role in the development of such structural elements. Traditional connection methods are possible, such as arc-welding and bolting, though these are typically less appropriate for thin materials, prioritising special techniques more suited to such materials. Established methods for connecting two elements of thin materials include self-drilling, self-tapping screws and blind rivets. When connecting a thin material to a thicker supporting member, fired pins are often used. A technology first utilised in the automotive industry, clinching, has recently been applied to building construction. Clinching is rapid, requires no additional componentry, and importantly causes no damage to galvanised or other metallic coatings. An even more recent innovation is the "Rosette", which is significantly stronger than clinching. 


\subsubsection{Connections}

A connection is defined as the physical component which mechanically fastens the structural elements and concentrates at the location where the fastening action occurs. Connections transfer the force and moment from a structural member into the supporting elements. A joint is defined as the connection plus the corresponding zone of interaction between the connected members and the panel zone of the column web. Structural joints can be classified into several categories, by referring to their strength and stiffness.

Connections for hot-rolled structures can be divided into either bolted or welded connections. Numerous joint profiles have been developed from these two basic components, and methodical design procedures for these joint profiles have long been in use by practicing engineers. According to Lennon et al. (1999), the choice of connecting technique to be employed for a particular cold-formed steel application would be influenced by several factors, including:

- The strength of the connection or connecting group at a discrete location in terms of

1. Initial small displacement response, and

2. Ductility.

- The availability of a generic analysis and design method.

- The reliability of the joint and availability of quality control methods.

- The cost of connection technique.

- Manoeuvrability of the joining equipment.

- Local environmental effects associated with the joining process, e.g. fumes from welding galvanised steel.

\subsubsection{Screw Connections}

Screws are a commonly utilised in cold-formed steel applications. Due to the thinness of the material, screw connections provide various advantages in terms of their simple design and expediency of installation. Screwed joints are convenient and efficient, and the only proviso being that the total thickness of the steel should not impair the self-drilling process. Screwed connections are said to either be loaded in tension, or loaded in shear; if the direction of the applied loads are either parallel, or perpendicular to the axis of the screw, respectively. These loading configurations are schematically represented in Figure 2-1. 


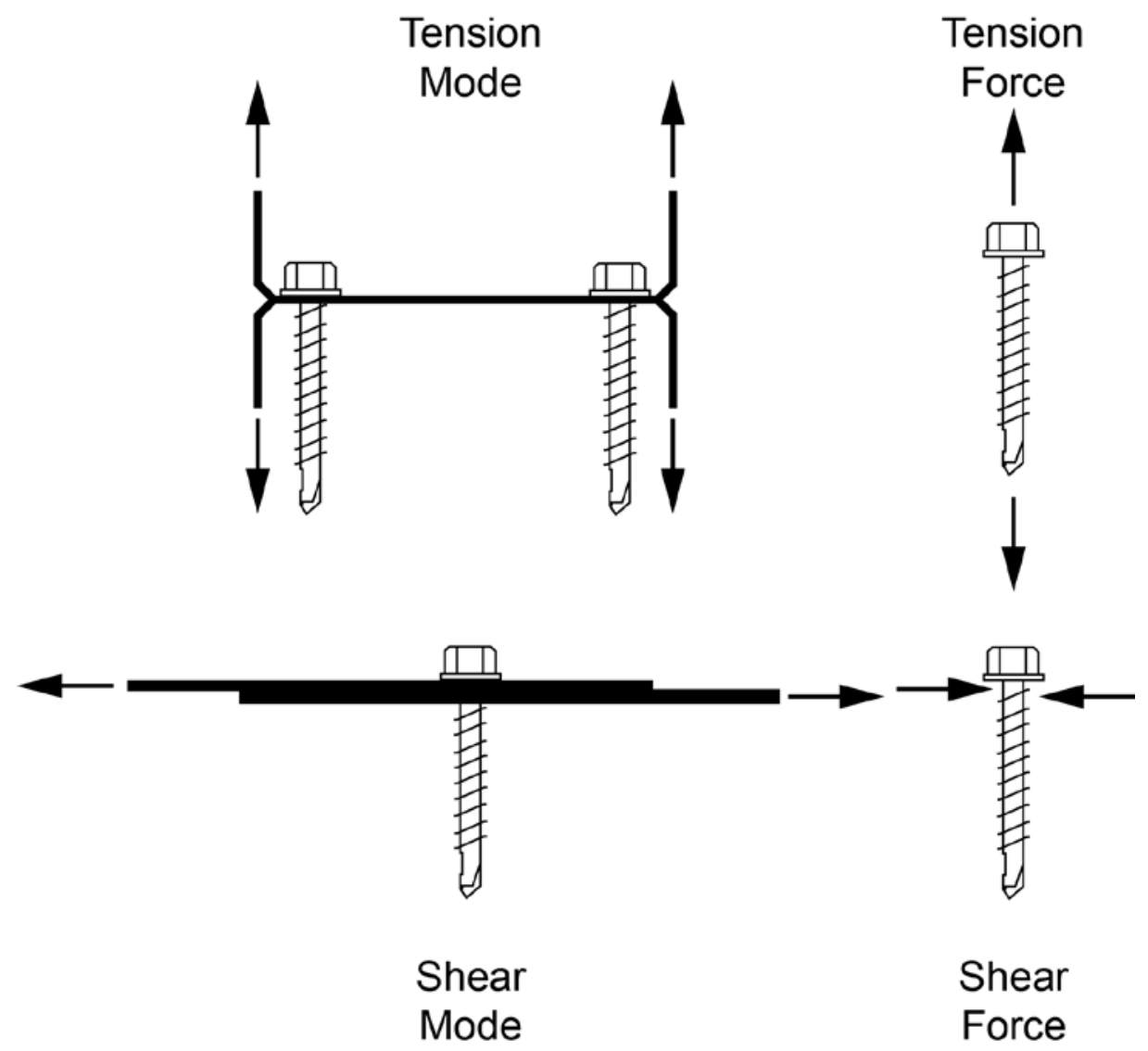

Figure 2-1 Screw loaded in shear and tension (Serrette \& Peyton, 2009)

The loading conditions of screw connections depend mostly on the application of the connecting structural elements, and in thin-walled members, according to Tomà et al. (1993), screw connections can be used for:

- Connecting steel sheets to their supporting structures, such as a purlin

- Interconnecting two or more sheets, such as longitudinal seams

- Assembling linear cold-formed sections, such as in storage racking

\subsubsection{Self-drilling screws}

The most prevalent means of fastening in modern light gauge steel construction, is through the utilisation of self-drilling screws. Such screws are externally threaded fasteners, which both drill their own hole and form threading in the steel sheets, as they are driven through with a screw gun. The screw tips essentially function as drill bits, drilling a hole through the steel before the screw threads engage the first layer of steel. This pre-drilled hole then ensures that the connecting layers of steel can be tightly clamped, once the screw is fully seated. Clamping of the layers is ensured, as the first layer cannot climb the threads ahead of the second or third layers. Self-drilling screws can connect steel sheets with a total thickness of up to $12.7 \mathrm{~mm}$. 
Self-drilling screws are composed of either carbon plated steel, with zinc for its anti-corrosive protection, or from stainless steel, with a carbon steel drill point. These screws are available in a variety of lengths, diameters, strengths and coatings. Selection of the screw diameter is based upon the required capacity of the connection, which increases with the diameter. The ASTM Standard C1513-04 (2007) states that, for steel tapping screws, the dimensions of selftapping screws can be obtained from the SAE J78 Standard (2013). The screw gauge number of a screw can be determined from the size of the outer thread diameter, and is provided in Table 2-1.

Table 2-1 Screw gauge number with corresponding screw diameter (SAE J78 , 2013)

\begin{tabular}{|c|c|}
\hline Screw Gauge Number & Screw Diameter (mm) \\
\hline 8 & 4.17 \\
\hline 10 & 4.83 \\
\hline 12 & 5.49 \\
\hline $14(1 / 4$ inch) & 6.35 \\
\hline
\end{tabular}

\subsection{COLD FORMED STEEL CONNECTION DESIGN PROVISIONS}

Screwed connections, especially those utilising self-tapping screws, provide an expeditious and effective solution to fastening sheet metal to framing members. Though Francka \& LaBoube (2010) examined the out-of-plane failure of screwed connections, namely screw pull-out failure, most researchers focus on the more complicated mode of in-plane shear failure of screwed connections.

Hancock et al. (2001) noted that the first provision of detailed guidance regarding the design of blind rivets and screw fasteners, was the ECCS document European Recommendations for the Design of Light Gauge Steel Members (1987), the background research of which was set out by Toma \& Stark (1978). Further developments and refinements of the European design rules have produced the Eurocode 3 Part 1.3 (2006). In order to apply the methods developed by the ECCS for screwed connections to U.S. practice, the formulae and results were recalibrated by Pekoz (1990), resulting in the development of the specific design rules currently used by the American Iron and Steel Institute Specification (AISI). The results of this study was based upon data collected from more than 3500 tests, conducted in the United States, Canada, Sweden and the Netherlands.

Research into these cold-formed steel connections, such as bolted and screwed connections, has been conducted by a number of scientists. The design rules for cold-formed steel structures has been specified in the North American Specification AISI S100 (2007), the Australia / New Zealand Standard AS/NZS 4600 (2005) as well as the Eurocode 3 (2006), and are widely utilised at present. An overview of the design equations used for the prediction of the connection capacities are provided in this section. 
The current design rules for cold-formed steel screwed connections are categorised by the different types of failure modes. These failure modes include; shear in screws, net section tension, connection shear limited by tilting and bearing, connection shear limited by end distance (tear out), tension in screws, pull-out and pull-over, as well as screws subjected to combined shear and pull-over. It was noted that Eurocode 3 (2006) requires that the thinnest sheet be adjacent the head of the screw, whereas AS/NZS 4600 (2005) and AISI S100 (2007) both allow for connections where the thinner sheet is not in contact with the screw head.

\subsubsection{Screws in Shear}

The failure of screws in shear is usually due to deficiency in the strength or quality of the screws, and is depicted in Figure 2-2. The nominal shear strength, $\left(P_{S S}\right)$, of a screw can either be determined by the manufacturer's report, or through AISI S100 (2007) specified testing. Furthermore, the nominal shear strength of the screw in the AS/NZS 4600 (2005) should reach the requirement given by the equation:

$$
P_{s s} \geq 1.25 P_{n s}
$$

Where $P_{n s}$ denotes the nominal connection shear strength, limited by both the tilting and bearing, as well as the end distance. The nominal shear strength of the screw, according to Eurocode 3 (2006), should be determined through testing, and should be a minimum of $20 \%$ higher than $P_{n s}$ and the nominal tensile strength of the net section of the screwed connection.

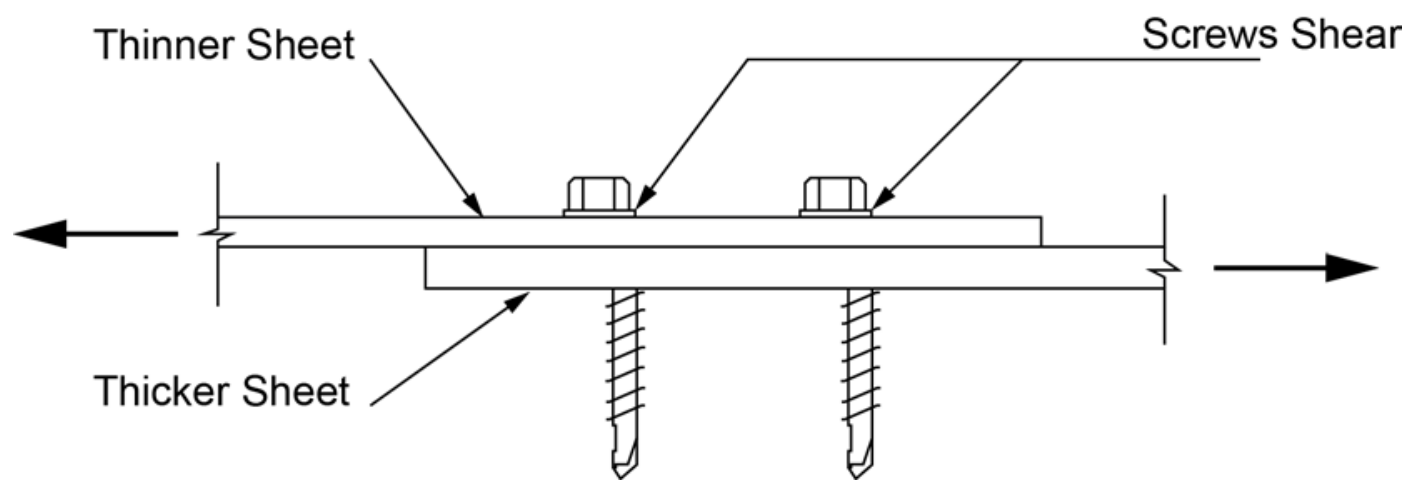

Figure 2-2 Shearing of screws (Yan \& Young, 2012)

\subsubsection{Net Section in Tension}

In the net section tension failure, cracking initiates from the screw holes and propagates along the cross section, as shown in Figure 2-3. Necking can normally be seen in the cross section of the plate. The nominal tensile strength, $P_{n}$, of the net section specified in both the AS/NZS 4600 (2005) and the AISI S100 (2007) are identical. The nominal tensile strength of the net section, for either a single screw, or row of screws perpendicular to the force, is given by 


$$
P_{n}=\left(2.5 \frac{d}{s}\right) A_{n} F_{u} \leq A_{n} F_{u}
$$

where $d$ is the nominal screw diameter; $s$ is the sheet width divided by the number of screw holes in the cross section being analysed; $A_{n}$ is the net area of the connected part; and $F_{u}$ is the tensile strength of the connected part being analysed. For multiple screws in the line parallel to the force, the nominal tensile strength of the net section is given by:

$$
P_{n}=A_{n} F_{u}
$$

The equation above is used directly in the Eurocode 3 (2006) to calculate the nominal tensile strength of the net section of screwed connection of thin sheet steels.

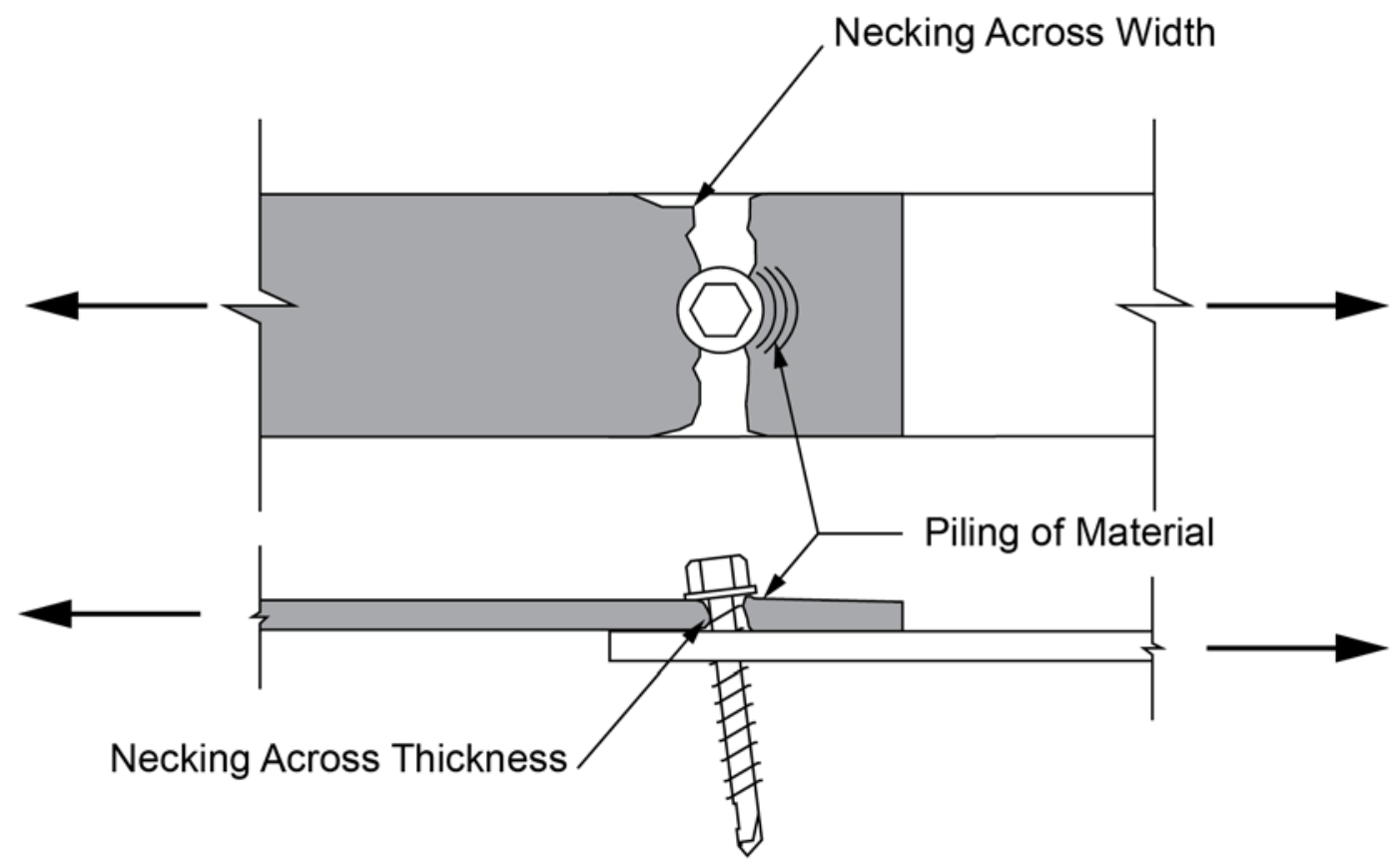

Figure 2-3 Net section tension failure (ECCS TC7 TWG 7.10, 2009)

\subsubsection{Tilting and Bearing}

The tilting and bearing failure is defined, in the AISI S100 (2007), as the failure of the connection in shear, limited by bearing and tilting. The bearing load, applied to the steel plate, produces screw tilting with respect to the steel sheet, the longitudinal tearing of the screw holes along the direction of force, and the bearing in front of the screws.

The design rules of the nominal shear strength per screw for the tilting and bearing failure, as specified in both the AS/NZS 4600 (2005), and the AISI S100 (2007), are typically similar. In cases where $t_{2} / t_{1} \leq 1.0$, the smallest value obtained from the following equations, will be taken as the nominal shear strength per screw: 


$$
\begin{gathered}
P_{n s}=4.2\left(t_{2}^{3} d\right)^{\frac{1}{2}} F_{u 2} \\
P_{n s}=C t_{1} d F_{u 1} \\
P_{n s}=C t_{2} d F_{u 2}
\end{gathered}
$$

Where the values of $t_{1}$ and $F_{u 1}$; and the values of $t_{2}$ and $F_{u 2}$; are the thicknesses and tensile strengths of the members contacting; and not contacting; the screw head or washer, respectively; with $C$ as the variable bearing coefficient. The AISI S100 (2007) requires that $C=2.7$ for shear connections, whilst in the AS/NZS 4600 (2005), the value of $C$ is determined according to the value of $d / t$, as listed in Table 2-2, where $t$ is the thickness of the connected part being analysed.

Table 2-2 Bearing Resistance Factor, C (AS/NZS 4600, 2005)

\begin{tabular}{|c|c|}
\hline $\boldsymbol{d} / \boldsymbol{t}$ & $\boldsymbol{C}$ \\
\hline$d / t<6$ & 2.7 \\
$6 \leq d / t \leq 13$ & $3.3-0.1 d / t$ \\
$d / t>13$ & 2 \\
\hline
\end{tabular}

For cases where $t_{2} / t_{1} \geq 2.5$, the nominal shear strength per screw can be determined as the smaller value of:

$$
\begin{aligned}
& P_{n s}=C t_{1} d F_{u 1} \\
& P_{n s}=C t_{2} d F_{u 2}
\end{aligned}
$$

For cases where $1.0<t_{2} / t_{1}<2.5, P_{n s}$ shall be determined through linear interpolation between the minimum values obtained from both aforementioned cases. The Eurocode 3 (2006) specifies that the nominal shear strength per screw, for tilting and bearing failure in screwed connections, to be:

$$
P_{n s}=\alpha F_{u} d t
$$

In which $\alpha$ is given by the following:

- If $t_{2} / t_{1}=1.0$ :

- If $t_{2} / t_{1} \geq 2.5$ and $t_{1}<1.0 \mathrm{~mm}$ :

- If $t_{2} / t_{1} \geq 2.5$ and $t_{1} \geq 1.0 \mathrm{~mm}$ :

- If $1.0<t_{2} / t_{1}<2.5$ : $\alpha=3.2 \sqrt{t_{1} / d} \leq 2.1$,

$\alpha=3.2 \sqrt{t_{1} / d} \leq 2.1$,

$\alpha=2.1$,

$\alpha$ obtained by linear interpolation 
It was determined by Rogers and Hancock (1997) that single overlap screwed steel sheet connections, which were loaded in shear, could fail in either one, or a combination, of several different failure modes. In order to appreciate the combined bearing/tilting failure mode, both pure bearing and pure tilting failure must be addressed first. In a pure bearing failure, as exhibited in Figure 2-4, an initial pull-out tear is observed in the direction of loading, with the screws remaining perpendicular to the steel sheet. Another feature of this failure mode is the piling of the steel sheet ahead of the screw.
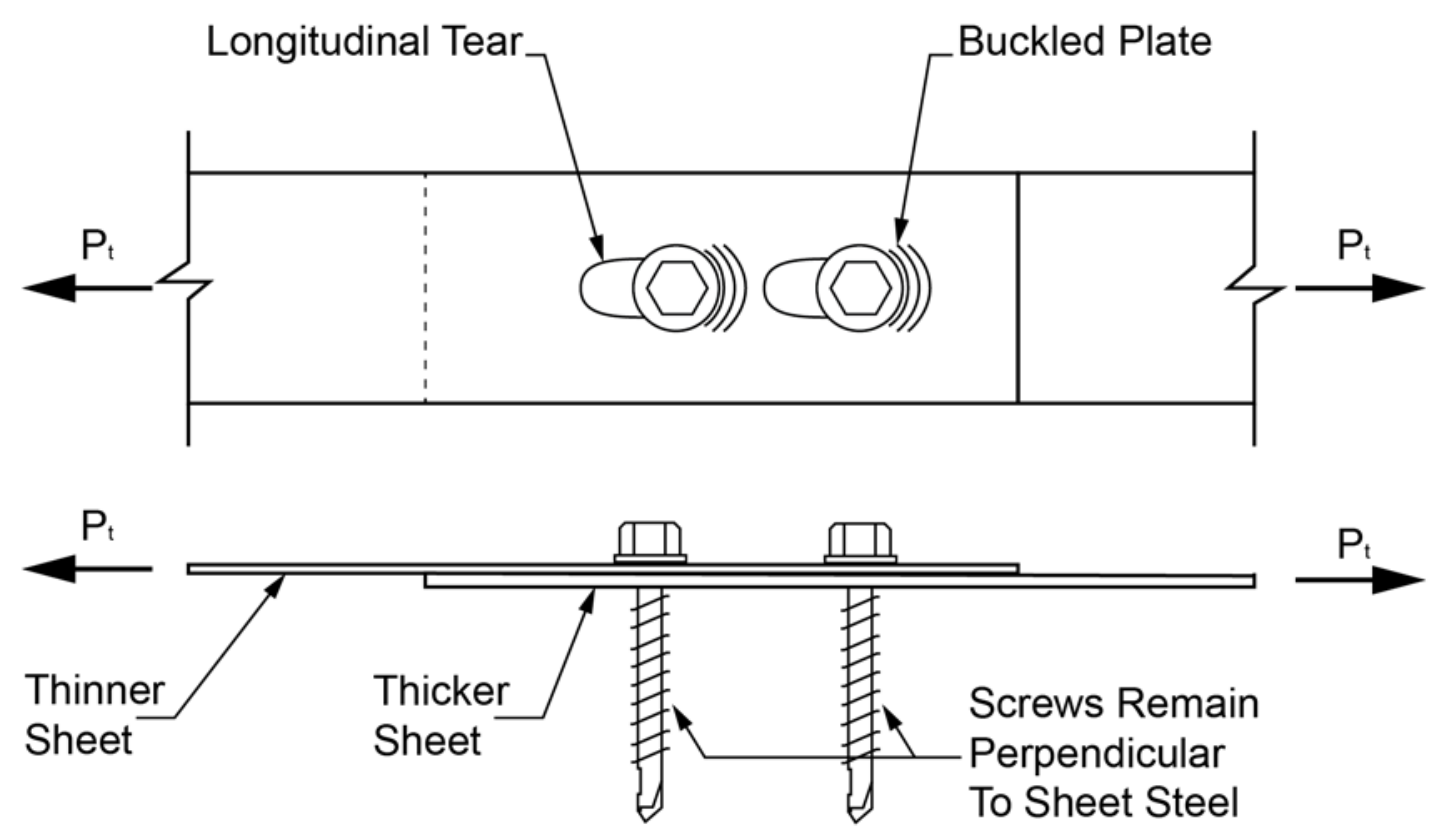

Figure 2-4 Bearing Failure (Rogers \& Hancock, 1997)

Rogers \& Hancock (1997) explained that tilting failure can be regarded as the failure of the screw itself, and attributed it to the eccentric shear load exerted on the screw by the adjacent steel sheets. Pure tilting failure may occur in a few select materials, exhibiting minimal distortion of the steel sheet adjacent the screw fastener, and is depicted in Figure 2-5 and Figure 2-6.

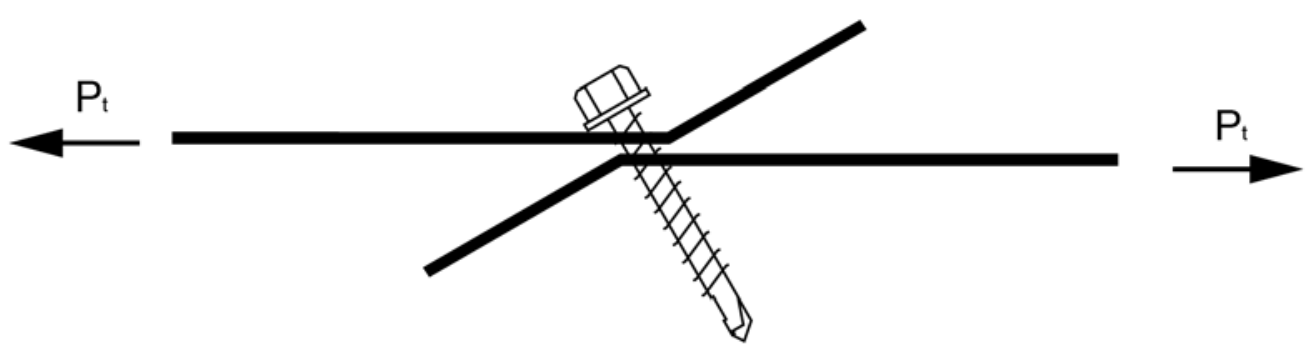

Figure 2-5 Tilting mode of failure (Serrette \& Peyton, 2009) 

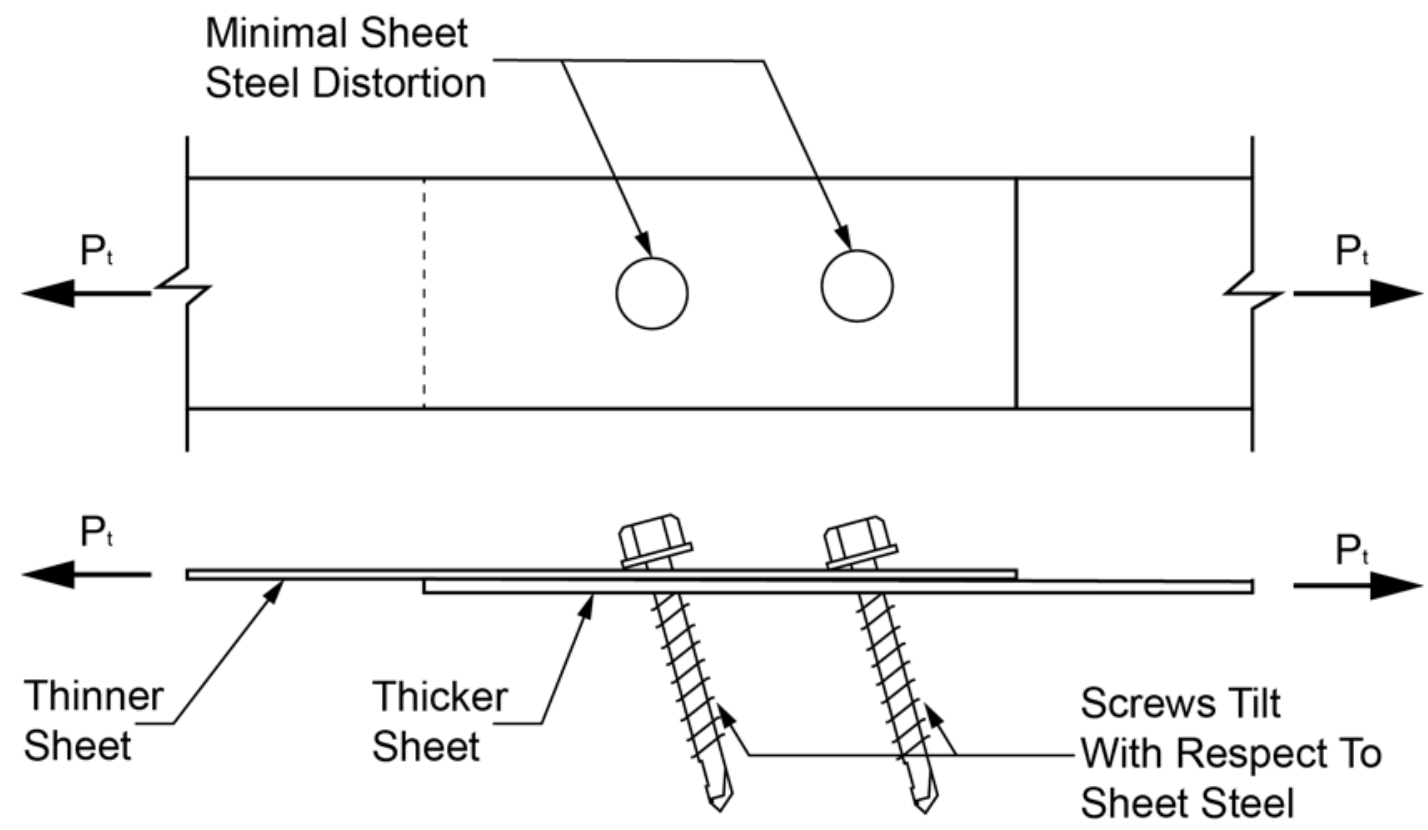

Figure 2-6 Tilting failure (Rogers \& Hancock, 1999)

A combination of bearing and tilting failure is shown in Figure 2-7, and exhibits significant screw tilting, due to the eccentric loading of the two sheets, with the ultimate load being preceded by piling of the steel sheet in front of the fasteners.
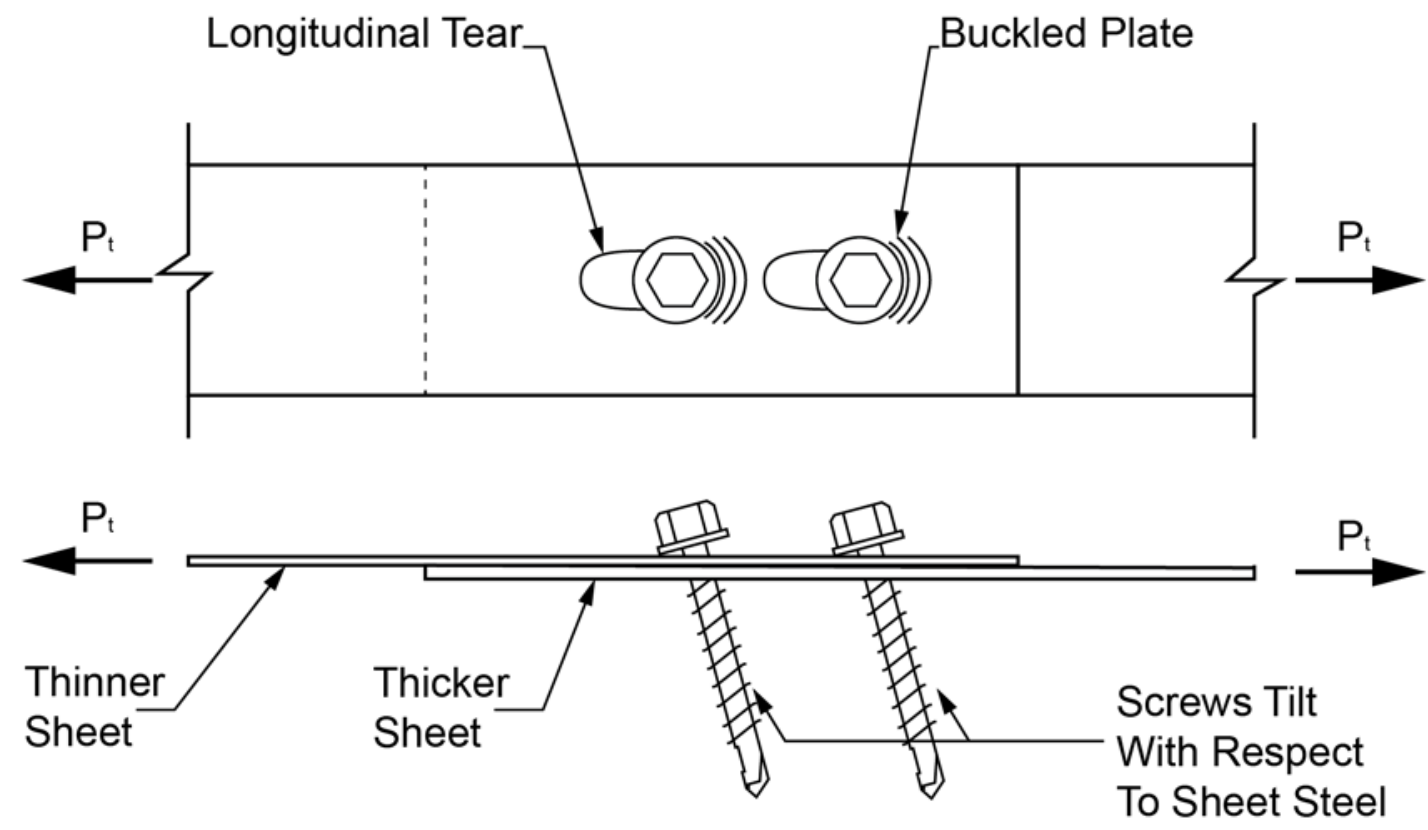

Figure 2-7 Bearing/Tilting failure (Rogers \& Hancock, 1997) 
An additional failure mode that could occur is pull-out failure, which was determined by Rogers and Hancock (1997) to occur as a result of rotation of the fastener with respect to the direction of the load, which causes an accumulation of tensile forces within the screw. Furthermore, this type of failure could manifest through a combination of bearing, pull-over or pull through in either of the connected elements, and is demonstrated in Figure 2-8

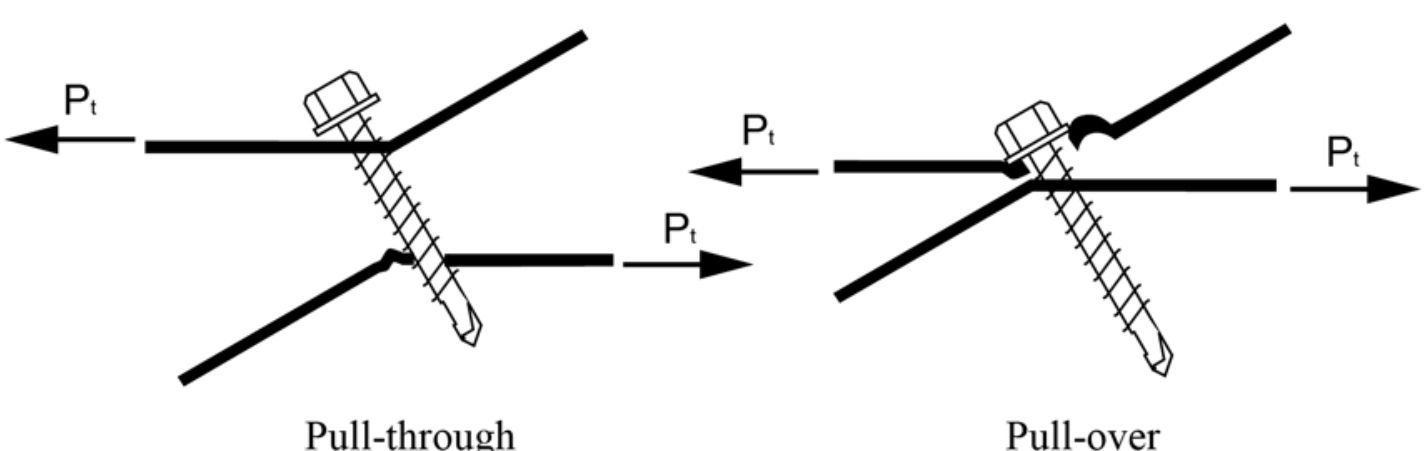

Figure 2-8 Bearing and pull-through or pull-over failure (Serrette \& Peyton, 2009)

\subsection{SCREW CONNECTION BEHAVIOUR}

Rogers and Hancock (1997) demonstrated that single overlap screwed steel sheet connections, loaded in shear, could fail in either one, or in a combination of several different modes, including; gross cross-section yielding, net cross-section fracture, end pull-out, bearing, tilting and screw shear. The behaviours observed through experimental testing of screwed connections are discussed in the section that follows.

\subsubsection{Effect of Steel Sheet Thickness}

Daudet and LaBoube (1996) investigated the shear behaviour of self-drilling screws utilised in low ductility steel through tests conducted on 264 samples. These tests comprised one and two screws in single shear, comparing the behaviour and performance of the self-drilling screws in normal and low ductility steels. The tests were conducted utilising No.10 and No.12 screws in steel sheets ranging in thickness from 0.74 to $2.49 \mathrm{~mm}$. Their research ascertained that screws subjected to single shear generally failed in one of two ways: the thinner steels, with $\mathrm{t} \leq 1.32 \mathrm{~mm}$, failed through tilting of the fastener combined with bearing of the screw hole; the thicker steels, where $t \geq 1.70 \mathrm{~mm}$, usually failed by shearing of the screw. 
Rogers and Hancock (1997; 1999) conducted research on the failure modes of self-drilling screwed connections, arranged parallel and perpendicular to the applied loads (refer to Appendix B for figure showing screw configurations). The study concluded that failure is more likely to be dependent upon tilting of the screws, if the connection is composed of two sheets of similar thickness, namely; thin G550 (see Appendix A) sheet steels ( $\mathrm{t}=0.42-1.00$ $\mathrm{mm})$, or G300 sheet steels $(\mathrm{t}=0.55-0.80 \mathrm{~mm})$. However, when two steel sheets of differing thicknesses are utilised, the thinner element will most likely fail in bearing distress. Additionally, Rogers and Hancock $(1997 ; 1999)$ stated that the mode of failure for all screwed connections tested in their study was a combination of bearing and tilting. The thinness of the steel sheets used contributed substantially to this governing mode of failure, and in some cases, the use of screws where the threads did not fully extend up to the base of the screw head. Thus, a non-threaded shank located directly below the screw head, due to limitations in the manufacturing process, could also affect the mode of failure.

Casafont et al. (2006) examined the influence that the thickness of the steel sheet exerted on the behaviour of screwed connections in single shear, conducting tests on sheet steels graded $\mathrm{G} 250$ and $\mathrm{G} 350(\mathrm{t}=0.85-3.00 \mathrm{~mm})$. Their results indicated that, if the adjoining sheets were of equal thickness; tilting always presented initially, followed by a period of joint elongation; and depending upon the number of screw columns utilised perpendicular to the load, the final failure mode would either be due to pull-out or net section failure. In cases where the pulling out process began, local plastification around the screw could be observed, concurring with the Rogers and Hancock’s (1997; 1999) description of pull-out failure.

Another observation was made, in that when the steel sheets differed in thickness, a variety of failures presented depending upon the configuration of the connection. Thinner sheets were once again placed in contact with the screw head, and in some cases, the same level of intensity for bearing and tilting presented during initial loading. However, joints utilising small diameter screws in order to connect sheets of significantly differing thicknesses together, were more prone to bearing. The ultimate mode of failure was again found to be dependent on the number of screws used in the connection, and occurred either through pullout, pull-through or net section failure.

\subsubsection{Effect of Steel Ductility}

In Daudet and LaBoube's (1996) investigation of single screws in single shear, all low ductility steels were cold reduced from normal ductility steels, with the degree of cold reduction ranging from $10-40 \%$ of the original thickness. The researchers found that the higher strength, low ductility cold reduced steel generally exhibited a higher shear capacity when compared to the normal ductility samples with the same gauge. They additionally noted that, prior to failure, both the normal and low ductility specimens generally exhibited the same amount of deflection, as indicated by the load-deflection graph presented in Figure 2-9. They concluded that, in general, all low and normal ductility single shear samples failing by screw bearing/tilting, failed in a very slow and ductile manner. 


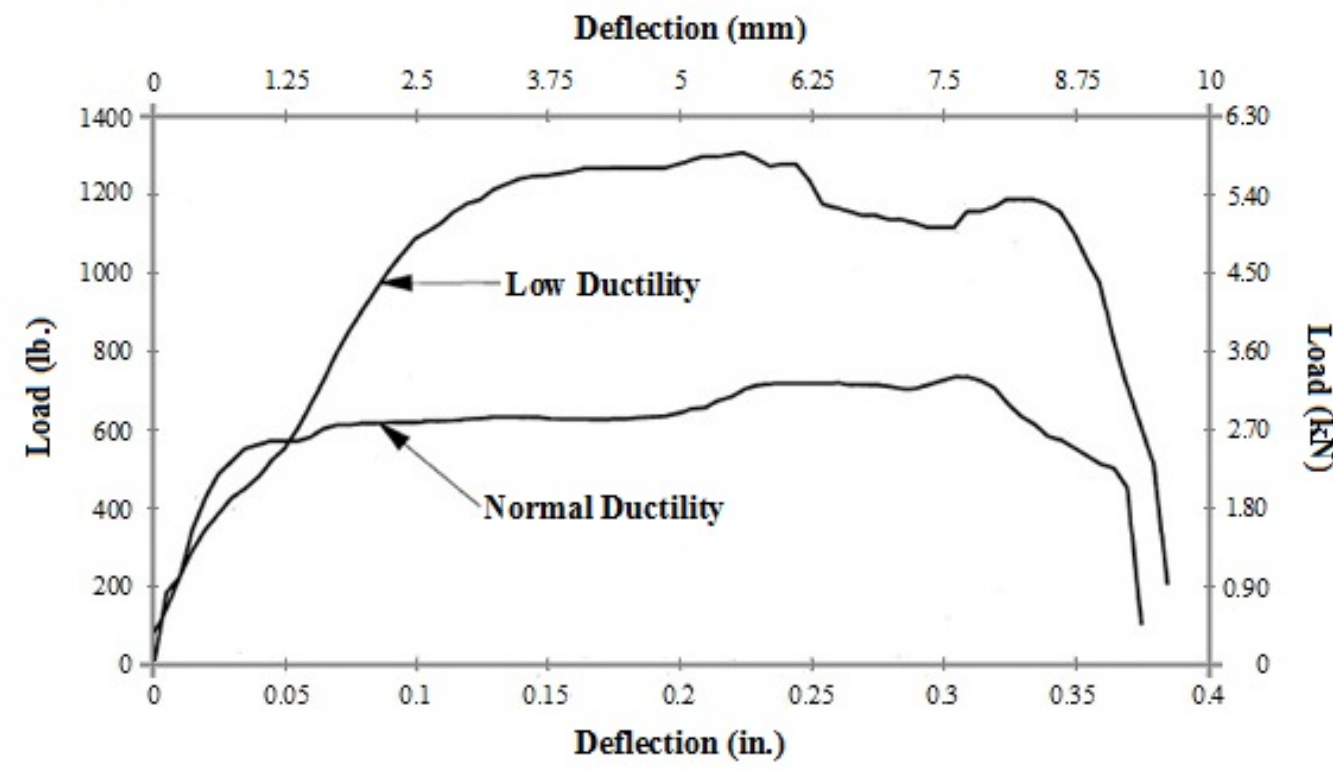

Figure 2-9 Load versus deflection for single screws in single shear for normal ductility steel $(t=$ $\left.1.04 \mathrm{~mm}, f_{y}=196 \mathrm{MPa}, f_{u}=336 \mathrm{MPa}\right)$ and low ductility steel $\left(t=1.09 \mathrm{~mm}, f_{y}=623 \mathrm{MPa}, f_{u}=673\right.$ MPa) (Daudet \& LaBoube, 1996)

Daudet and LaBoube's (1996) research also explained that, when the strength of the screw is the governing factor; such as exhibited in thicker steel samples $(\mathrm{t} \geq 1.70 \mathrm{~mm})$, where failure occurs through shearing of the screw; that prior to ultimate failure, the same amount of shear capacity and deflection is experienced by both the normal and low ductility specimens. This behaviour is illustrated by the load-deflection graph in Figure 2-10. The researchers postulated that low and normal ductility single shear specimens failing by screw shearing typically failed in a fairly sudden, non-ductile manner.

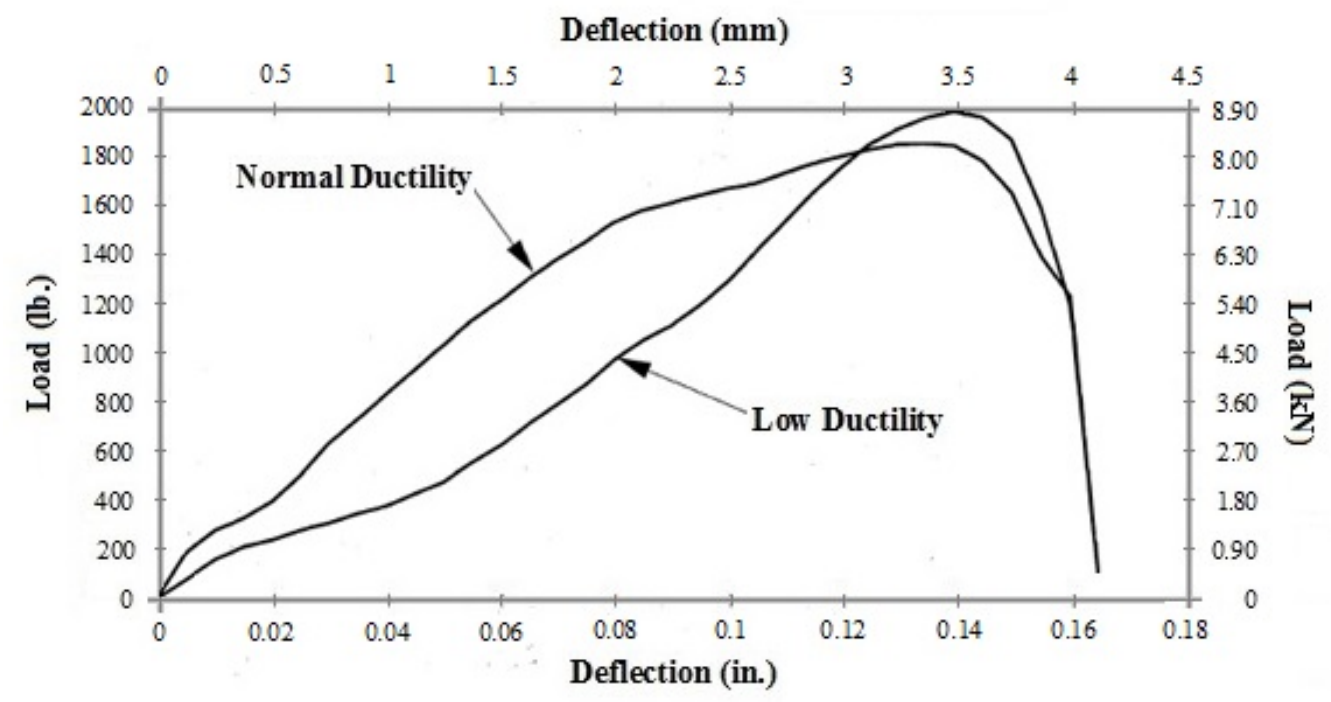

Figure 2-10 Load versus deflection for single screws in single shear for normal ductility steel $(t=$ $\left.1.83 \mathrm{~mm}, f_{y}=403 \mathrm{MPa}, f_{u}=458 \mathrm{MPa}\right)$ and low ductility steel $\left(t=1.73 \mathrm{~mm}, f_{y}=746 \mathrm{MPa}, f_{u}=784\right.$ MPa) (Daudet \& LaBoube, 1996) 


\subsubsection{Effect of Number of Screws}

Daudet and LaBoube (1996) also studied the behaviour of multiple fasteners, through testing single and double screw connections in single shear, where the screws were arranged parallel to the applied load. Their research indicated that double screws behaved similarly to single screws in single shear, where failure was deemed to occur through a combination of bearing and tilting. The results of their study only included single and double screw connections, and the failure modes for connections utilising multiple screws has not yet been established.

Koka et al. (1997) conducted tests on the behaviour of screw connections, using self-drilling screws in low ductility steel, by testing 21 samples to failure. Their tests comprised of screw connections arranged parallel to the applied load, connecting sheets of identical thicknesses, either 0.43 or $0.74 \mathrm{~mm}$. The results of their preliminary study concluded that, for screws in single shear, the governing mode of failure was again found to be through a combination of tilting and bearing.

Koka et al. (1997) described the onset of failure to occur at approximately $75 \%$ of the ultimate capacity, followed by significant tilting of the screws around $85 \%$ of the ultimate capacity. Subsequent sheet separation occurred at around $90 \%$ of the ultimate capacity, due to slippage of the drill tip out of the enlarged hole, which occurred thread by thread until the ultimate capacity of the screw was attained, finally resulting in a combined tilting/bearing failure. When using multiple columns of screws, the researchers noted that the first column incurred the greatest amount of deformation when compared to the subsequent columns. From their findings, the conclusion could be made that a screwed connection, consisting of four screws or less arranged longitudinally to the applied load, will exhibit a tilting/bearing failure.

Casafont et al. (2006) examined the modes of failure of screwed connections, through variation of the number of screws and the thickness of the steel sheet. The influence of the number of screws used was examined by variation of the number of screw columns, where each column consisted of two screws arranged perpendicular to the applied load, as depicted in Figure 2-11.

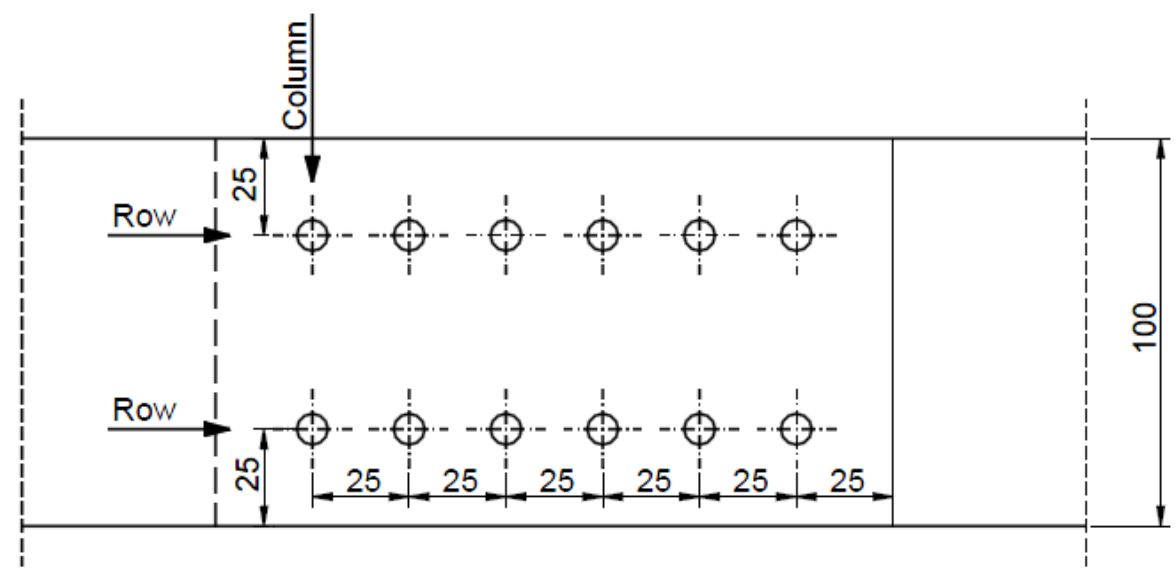

Figure 2-11 Configuration for test specimens (Casafont et al., 2006) 
Their results suggested that the onset of failure was dependent upon both the sheet thickness and the type of screw used, though the final failure mode would depend on the number of screws used. In samples where identical sheet thicknesses were used, the connections with four or less columns resulted in pull-out of the screws, as shown in Figure 2-12(a). When six or more columns were utilised, failure of the net section was ensured, seen in Figure 2-12(b). Where the thicknesses of the sheets were varied, Casafont et al. (2006) concluded that one column would undergo pull-out failure, six columns or more would ensure net section failure and an intermediate number of columns could result in pull-out, pull-through or net section failure, shown in Figure 2-13.

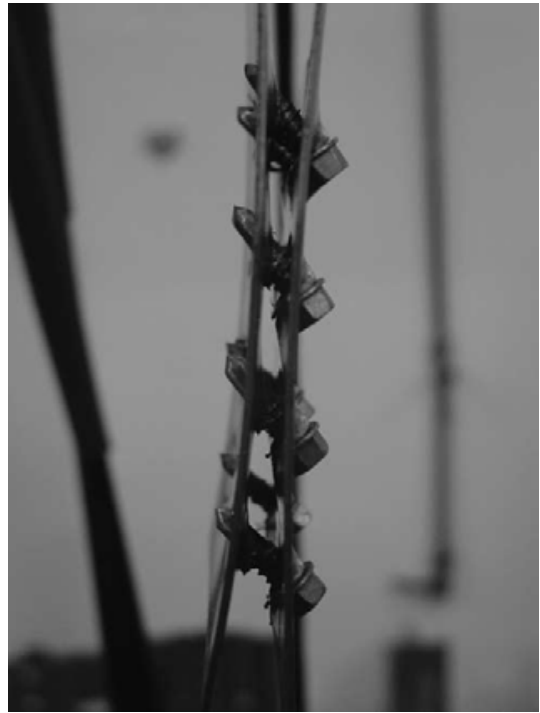

(a)

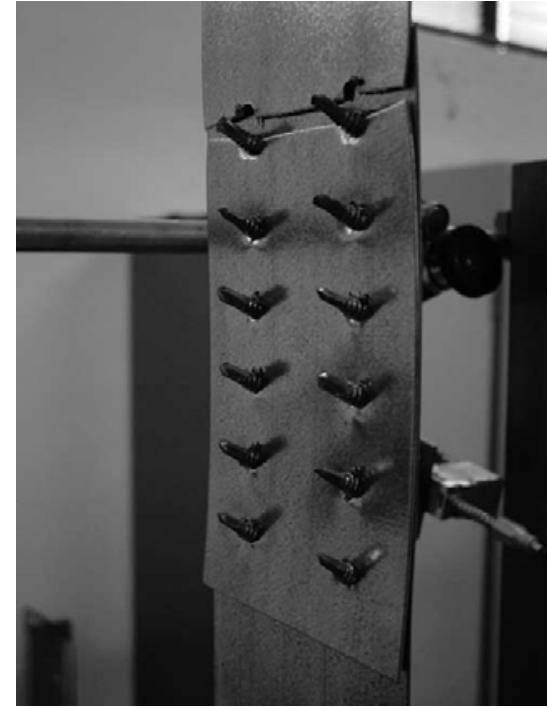

(b)

Figure 2-12 Failure modes for sheets of identical thickness (a) Tilting \& Pull-out; (b) Tilting \& Net Section (Casafont et al., 2006)

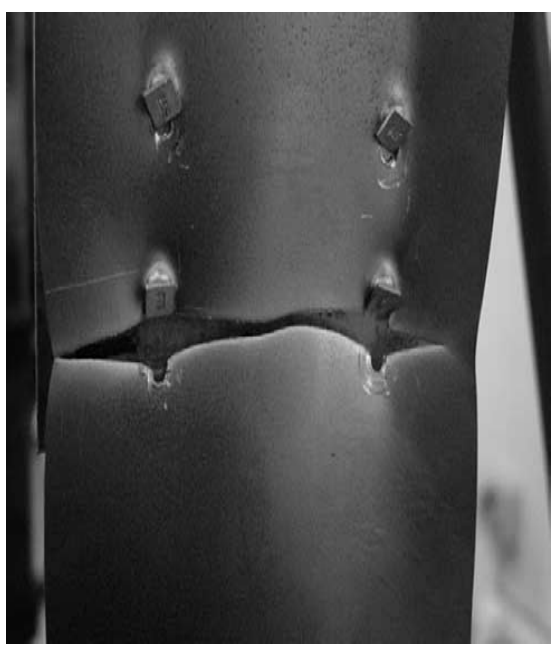

(a)

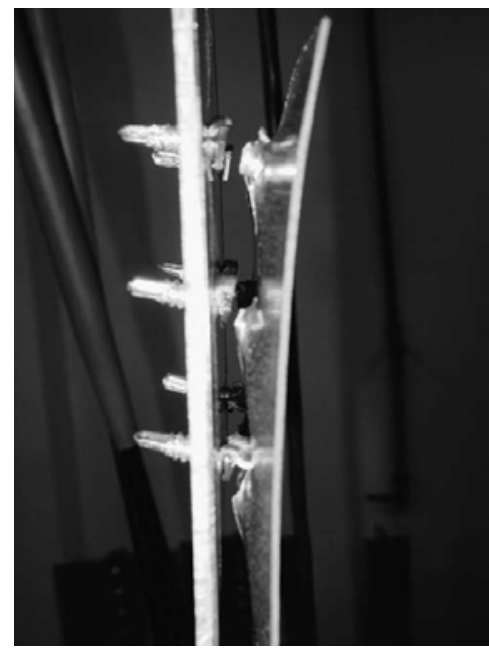

(b)

Figure 2-13 Failure modes of sheets of different thickness (a) Tilting, Bearing \& Net section; (b) Tilting, Bearing \& Pull-through (Casafont et al., 2006) 
Casafont et al. (2006) concluded that all test specimens quintessentially failed by two modes of failure:

- $\quad \mathrm{T}+\mathrm{B}+\mathrm{PO}$ : Tilting + Bearing + Pull-Out (or pull-through), or

- $\mathrm{T}+\mathrm{NSF}$ : Tilting (and bearing) + Net Section Failure.

Supplementary to the physical characteristics defined above, the failure modes were also observed with reference to their Force against Displacement curves (F-d curves). Two types of F-d curves were obtained and an example of these are given in Figure 2-14. The T+NSF failure curve presents with three behavioural stages: elastic behaviour, hardening and failure. The $\mathrm{T}+\mathrm{B}+\mathrm{PO}$ curve also consists of three stages, though they are not as apparent due to the gradual nature of both yielding and failure. Casafont et al. (2006) interpreted that the influence exerted by the number of screws utilised on the ultimate behaviour of the connection, can be observed on both the test specimens themselves, and also through analysis of the F-d graphs obtained.

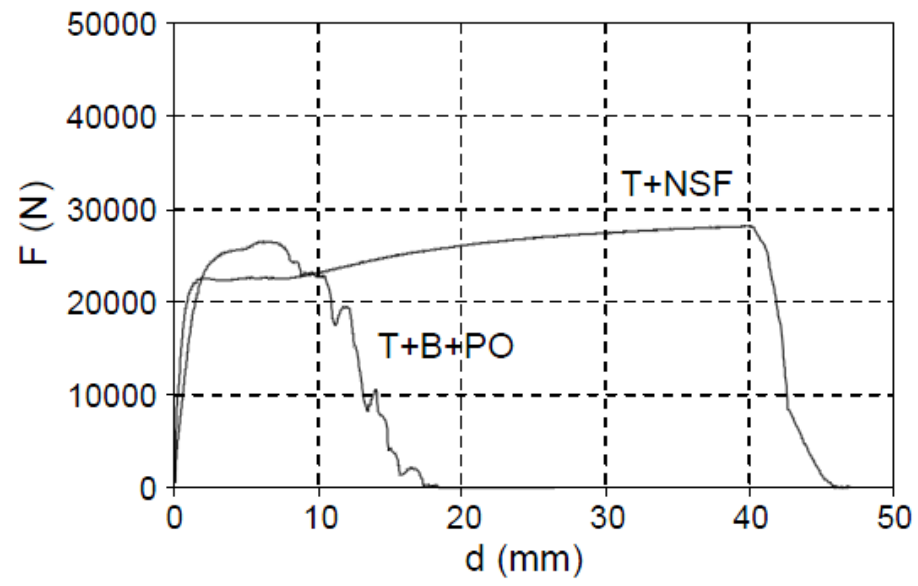

Figure 2-14 Load vs. displacement curves for T+NSF \& T+B+PO failure modes (Casafont et al., 2006)

Li et al. (2010) came to a similar conclusion through their tests of 75 single lap screw connections, using normal ductility $1.00 \mathrm{~mm}$ steel sheets. They observed that; for connections where the number of screws used was low, failure occurred by tilting/bearing (Figure 2-15 (a) and (b)), and where a large number of screws were used, net section failure occurred (Figure 2-15 (c)). In their study, a large number of screws comprised a connection of nine screws, arranged into three rows and three columns. They found that for connections where several columns of screws were used, failure almost always occurred in the column closest to the jaws of the testing machine; and sheet fracturing invariably occurred in the sheet that exhibited exposure of the screw threads. All specimens which failed by fracture of the sheets had multiple screw lines perpendicular to the applied load. 


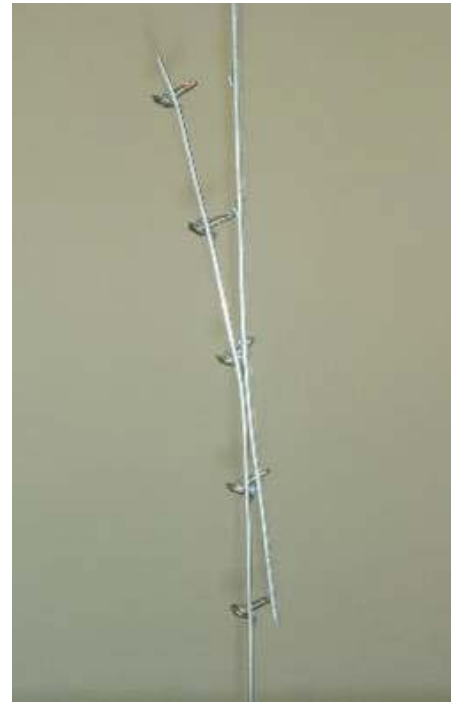

(a)

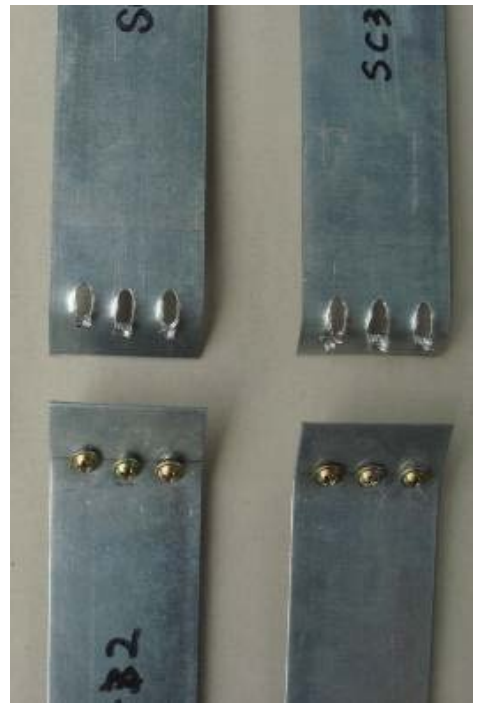

(b)

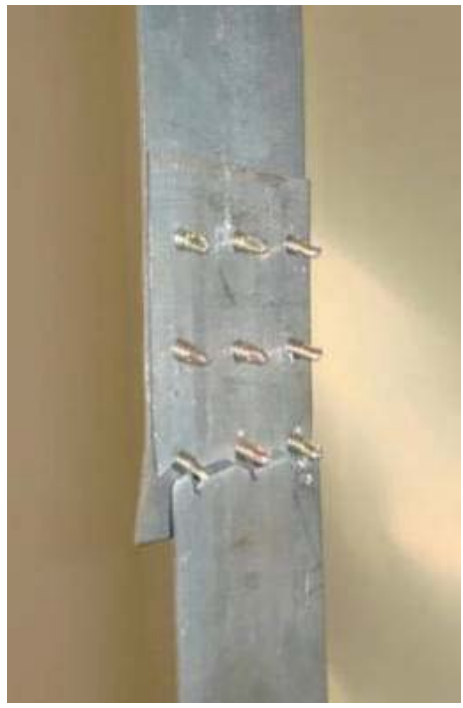

(c)

Figure 2-15 Failure modes of screw connections (a) Tilting; (b) Bearing; (c) Steel sheets fracture (Li et al., 2010)

\subsection{SCREW CONNECTION STRENGTH}

The ultimate strength of the screw connections, and their relative performance, are subject to numerous factors, including; the number of screws used, the patterning of the screws, their spacing, end distance and the type of screw used. The following section provides an overview of previous studies, in order to determine the influence of some of these factors on the shear connection strength of screwed connections.

\subsubsection{Effect of Number of Screws on Connection Strength}

Previous studies have shown that the number of screws used in a connection has a definite influence on the strength of said connections, whereby an increase in number of screws will increase the connection shear strength of the screws. Current design equations are based on a single screw connection, and the strength of multiple screw connections is extrapolated as a direct multiple of the single screwed connection strength. This assumption has been rebuked in several studies, which will be discussed in the following section, where it is revealed that the connection strength does not increase proportionally to the number of screws used in the connection; but rather that the connection strength per screw diminishes as the number of screws increases. 
Daudet \& LaBoube (1996) broadened their research on the use of multiple fasteners and its influence on the connection strength. Connections were fabricated using normal and low ductility sheet steel, including single and double screw connections, arranged parallel to the applied load, in shear. They observed that the use of two screws did not necessarily double the shear capacity of the connection, as was previously assumed; and when multiple fasteners were utilised, the deformation produced secondary stresses on the connections, resulting in a reduction of the connection performance.

It was further observed that the connection deformations associated with double screw connections using low ductility steel, was somewhat less than those of normal ductility connections. This was attributed to the fact that low ductility specimens achieved $99 \%$ of their expected capacity, while the normal ductility specimens only experienced $90 \%$ of the expected capacity. Daudet \& LaBoube (1996) expressed that it was somewhat ironic to observe that the low ductility steel; which possessed lower $F_{u} / F_{y}$ ratios, and therefore less stress redistribution capacity; outperformed normal ductility steel. They also noted that the reduced performance of normal versus low ductility material was potentially as a result of greater sheet separation that took place with normal ductility specimens. The increase in sheet separation may have produced an increase in secondary effects in the normally ductile material, which could lead to slightly premature failure.

Serrette \& López (1996) studied the performance of self-tapping screws in lap-shear metal-tometal connections, including connections where the fasteners were both properly installed, and those where the the fasteners were stripped. The No.12 screw was utilised in all the fabricated connections, except for a difference in the head style, and the thread style in one example. Both two and four screw connections were considered, and the screw patterns, including the end, edge and centre-to-centre dimensions were determined based upon the AISI recommendations.

The results of their study concluded that the strength per screw decreased as the number of screws in the connection increased. The maximum load capacities for two and four screwed connections are provided in Table 2-3, where it can be seen that for all screw types considered, the ultimate connection strength per screw for the two screw configuration (X2 \& $\mathrm{X} 2 \mathrm{~S}$ ) was greater than that of the four screw connection (X4 \& X4S). This is characterised by the ratios for the normal screw connections (X2/X4) and the stripped screw connections (X2S/X4S) all being greater than 1.0. The researchers suggested that, when comparing the $\mathrm{X} 2$ and $\mathrm{X} 4$ series results, any benefits derived from special screw designs may not be fully realised in connections where the spacing is close, and that the maximum load ratios for the stripped screws were approximately the same. 
Table 2-3 Comparison of maximum load capacities (per screw) for 2-screw and 4-screw connections (Serrette \& López, 1996)

\begin{tabular}{|c|c|c|}
\hline \multirow{2}{*}{ Screw Type } & $\begin{array}{c}\text { Maximum Load Ratio } \\
\text { X2/X4 }\end{array}$ & $\begin{array}{c}\text { Maximum Load Ratio } \\
\text { X2S/X4S }\end{array}$ \\
\hline A & 1.02 & 1.03 \\
\hline B & 1.07 & 1.04 \\
\hline C & 1.10 & 1.04 \\
\hline
\end{tabular}

Note: Type A - Pancake head; Type B - Hexagon washer head; Type C - Hexagon washer head with large washer (Refer to Appendix B for screw head description)

LaBoube \& Sokol (2002) investigated how the strength of screwed connections, using the same thickness normal ductility steel, was influenced by the fastener patterns, screw spacing, stripped screws and the number of screws. Currently accepted design practice assumes that if a connection consists of four screws, it should be four times as strong as a connection comprising only one screw, provided that the adjoining sheets do not fracture first.

LaBoube \& Sokol (2002) confirmed that the strength per screw in a connection diminishes as the number of screws increases. This demonstrated that although a linear relationship exists between the number of screws and the connection strength, when compared to the connection strength of a single screw, the strength did not increase as a direct multiple of the number of screws utilised in the connection (Figure 2-16). This can be further explained by the following example - a single screw's connection strength is approximately $2.5 \mathrm{kN}$, thus a connection comprising 4 screws should have a connection strength of approximately $10 \mathrm{kN}$, however, the value obtained is nearer to $8 \mathrm{kN}$.

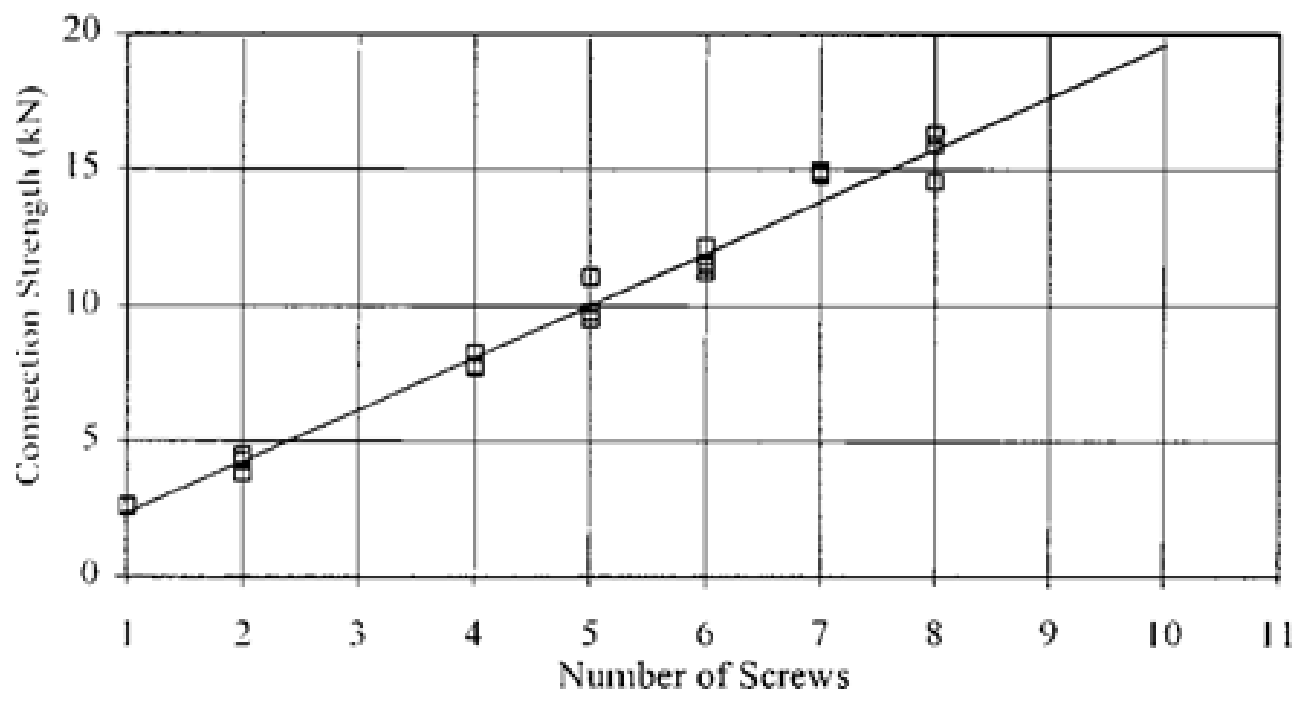

Figure 2-16 Effect of number of screws on connection strength ( $t=0.9 \mathrm{~mm}$, No.12 Screws) (LaBoube \& Sokol, 2002) 
This decrease in connection strength is defined as the "Group Effect", and illustrates the ability of a group of fasteners to share a load. The "Group Effect" is defined as the ratio of the connection strength per screw to the average strength for a single screw connection of the same sheet thickness and screw size (LaBoube \& Sokol, 2002). The relationship between the "Group Effect" and the number of screws can be seen in Figure 2-17 where the strength per screw diminishes as the number of screws are increased. The graph shows that if all the screws acted and contributed equally to the connection, the "Group Effect" would be 1.0.

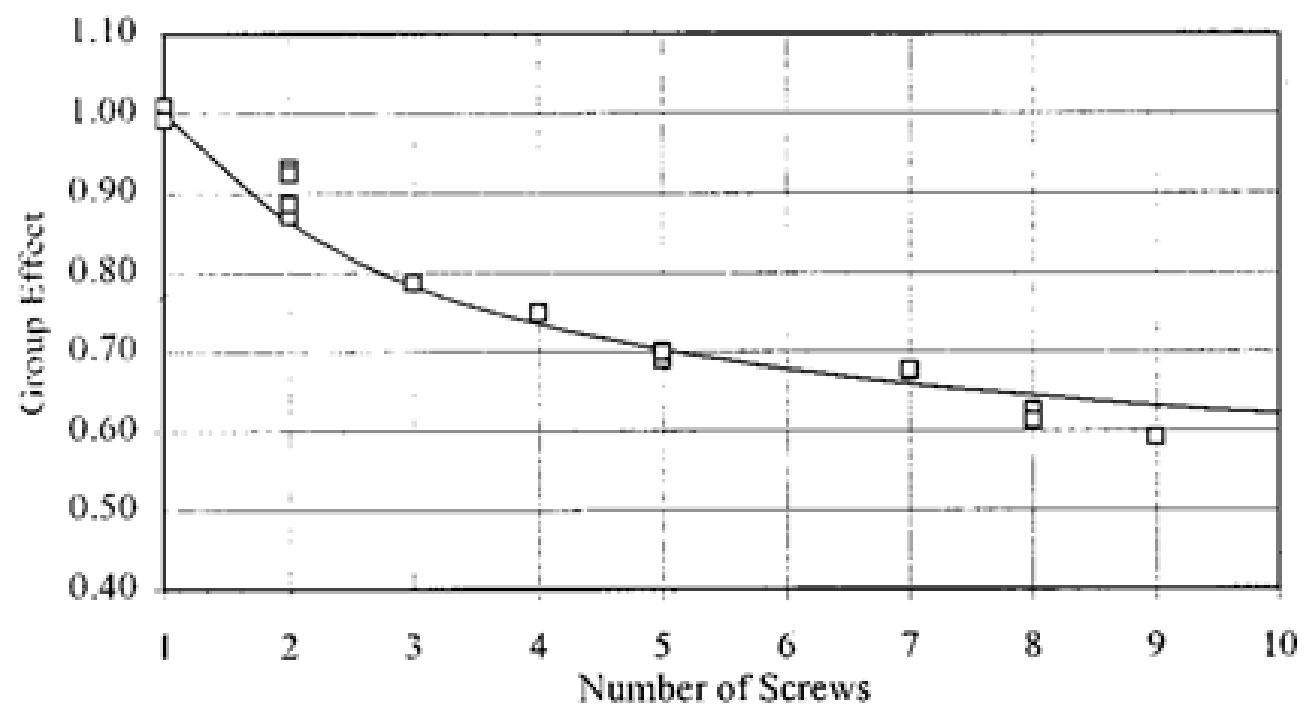

Figure 2-17 Group effect versus number of screws $(t=1.5 \mathrm{~mm}$, No. 10 screws) (LaBoube \& Sokol, 2002)

Li et al. (2010), obtained comparable results through their research into the shear behaviour of screw connections in cold-formed steel. The number of screws used in their connections varied from two to five, and the screws were arranged longitudinally (parallel) to the applied load, whist the spacing was kept a constant three times the diameter of the screw. The results of the tests are presented in Table 2-4; where $\mathrm{P}$ is the mean shear strength, $\mathrm{P} / \mathrm{P}_{1}$ is the ratio of the mean shear strength to the single screw shear strength, and the ratio per screw essentially the "Group Effect" for the connections. Once again, Table 2-4 illustrates how the strength per screw in the connection reduces as the number of screws arranged parallel to the applied load increases. 
Table 2-4 Effect of number of screws on shear strength ( $\mathrm{Li}$ et al., 2010)

\begin{tabular}{|c|c|c|c|}
\hline Specimen & $\mathbf{P}(\mathbf{k N})$ & $\mathbf{P} / \mathbf{P}_{\mathbf{1}}$ & $\mathbf{P}_{\mathbf{p e r ~ s c r e w}} / \mathbf{P}_{\mathbf{1}}$ \\
\hline SC1-3D & 3.044 & 1.00 & 1.000 \\
\hline SC2-3D-L & 5.468 & 1.80 & 0.898 \\
\hline SC3-3D-L & 7.731 & 2.54 & 0.847 \\
\hline SC4-3D-L & 10.286 & 3.38 & 0.845 \\
\hline SC5-3D-L & 11.810 & 3.88 & 0.776 \\
\hline
\end{tabular}

Koka et al. (1997) conducted a preliminary study investigating the behaviour of screw connections in identically thick, low ductility steel, through arrangement of the screws parallel to the applied load. The results for the screwed connections in single shear demonstrated that the load distribution on a screw group is neither linear nor equal, as is currently assumed in the design codes. This is graphically illustrated in the load versus number of screws plot in Figure 2-18, where it is observed that the curve flattens at the top for a connection with four screws. Thus, the strength of the screw connection increases proportionally when the number of screws used was less than or equal to three. It can be postulated that the use of low ductility steel does not decreases the strength per screw, unless a large number of screws are utilised in the connection.

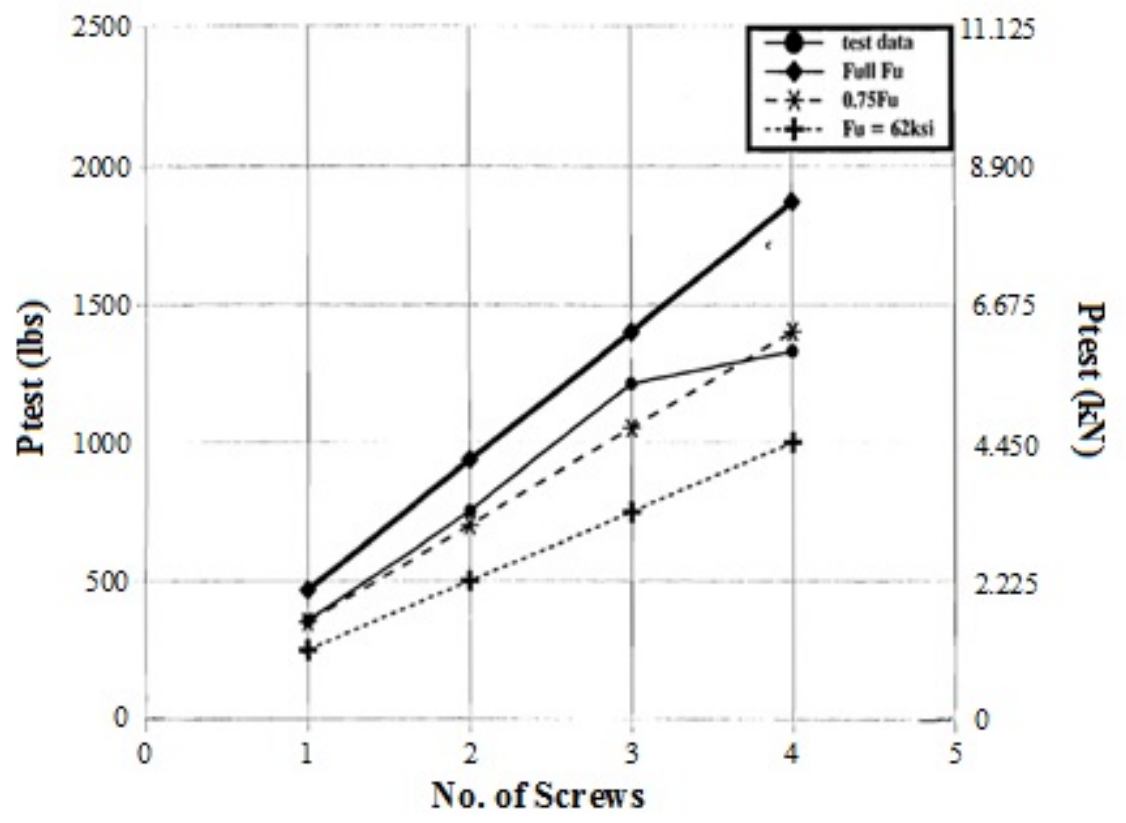

Figure 2-18 Load versus number of screws (Koka et al., 1997) 
Casafont et al. (2006) researched screwed connections between two straps, using steel grades of either S350 GD+Z or S250 GD+Z, where the nominal (denoted ' $n$ ') and tested (denoted ' $\mathrm{t}$ ') mechanical properties are shown in Table 2-5. The influence of the number of screws, as well as the thickness of the sheet steel, was investigated by testing single lap screws connections in shear, consisting of identical and varying sheet steel thicknesses. The influence of the number of screws was examined by varying the number of screw rows, where a row consisted of two screws perpendicular to the applied load, seen in Figure 2-11.

Table 2-5 Steel Mechanical Properties (Casafont et al., 2006)

\begin{tabular}{|c|c|c|c|c|c|}
\hline Steel & $\mathrm{f}_{\mathrm{y}, \mathrm{n}}(\mathrm{MPa})$ & $\mathrm{f}_{\mathrm{u}, \mathrm{n}}(\mathrm{MPa})$ & $\mathrm{t}(\mathrm{mm})$ & $\mathrm{f}_{\mathrm{y}, \mathrm{t}}(\mathrm{MPa})$ & $\mathrm{f}_{\mathrm{u}, \mathrm{t}}(\mathrm{MPa})$ \\
\hline \multirow{3}{*}{ S350 GD+Z } & \multirow{3}{*}{350} & \multirow{3}{*}{420} & 1 & 392 & 520 \\
\hline & & & 1.5 & 387 & 519 \\
\hline & & & 3 & 385 & 512 \\
\hline \multirow{3}{*}{ S250 GD+Z } & \multirow{3}{*}{250} & \multirow{3}{*}{330} & 0.85 & 285 & 345 \\
\hline & & & 1 & 303 & 393 \\
\hline & & & 1.5 & 317 & 391 \\
\hline
\end{tabular}

Casafont et al. (2006), drew two conclusions regarding the experimental ultimate loads of all the connections tested. Firstly, parameters such as the type of screw head, whether hexagonal or square, and the shaft length did not significantly affect the strength of the specimens. Secondly, the joints tested in the study did not clearly exhibit "Group Effect". Through comparison of the experimental bearing strength of connections consisting of six to eight screws versus two screw connections, an increase in the number of screws did not induce a decrease in strength per screw, as depicted in Figure 2-19.

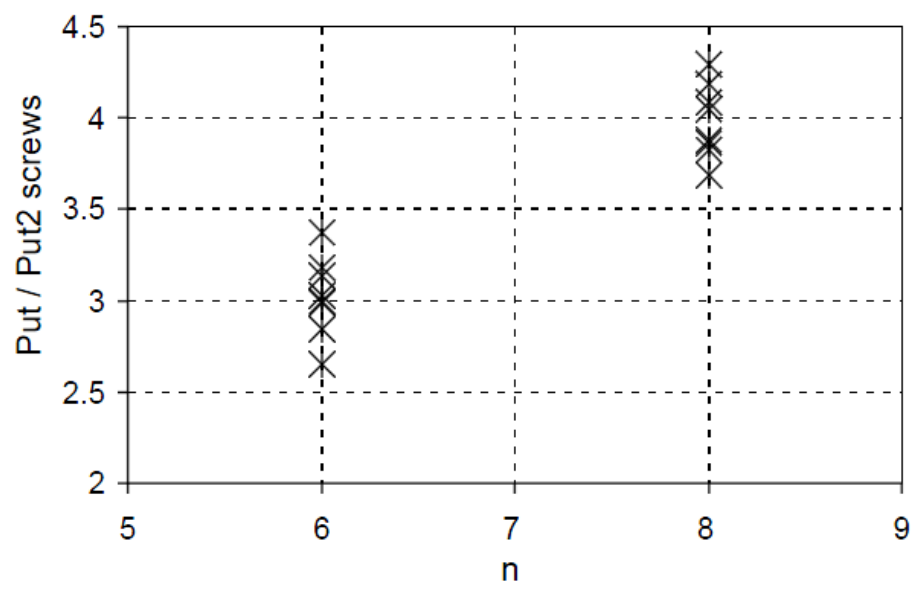

Figure 2-19 Ratio $P_{u t} / P_{u t}$ 2-screws versus number of screws (Casafont et al., 2006) 


\subsubsection{Effect of Screw Patterns on Connection Strength}

The screws in a connection can be arranged in myriad patterns, and are in essence defined by the number of rows and columns in the connection. The columns are specified as a linear configuration arranged transversely, perpendicular to the direction of loading, where a row would be defined as a line of screws arranged longitudinally, parallel to the direction of the applied load.

The current assertion is that the pattern of the screws in a connection does not significantly affect the strength, though an increase in the number of columns would increase the connection strength. Sokol et al. (1998) conducted research into what influence the number of screws, their spacing and geometric patterning would have on the connection strength of normal ductility steels. The experiment consisted of 27 different geometric patterns, of which the differing four screw patterns are shown in Figure 2-20, along with their experimental results in Table 2-6, as well as the calculated group effect, arranged in order of increasing strength.

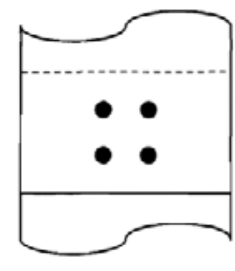

$4 \mathrm{~A}$

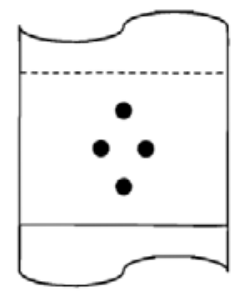

4B
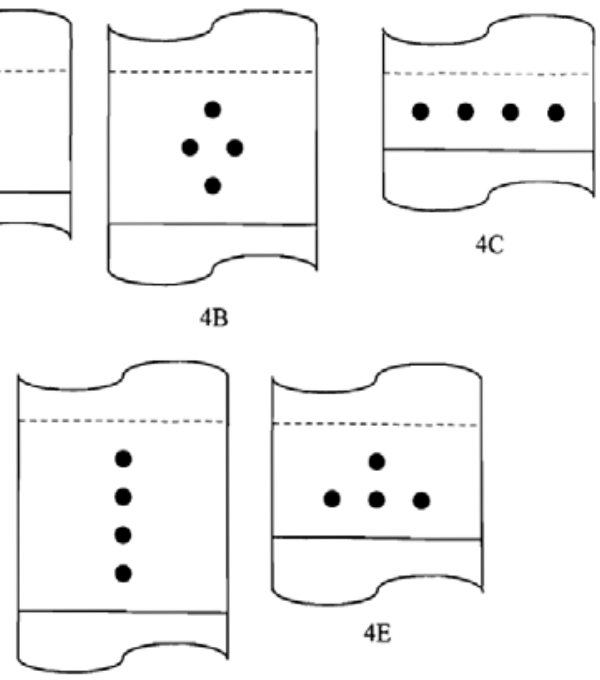

$4 \mathrm{D}$

Figure 2-20 Screw Patterns for Four Screws (Sokol et al., 1998)

Table 2-6 Typical Results for Four Screw Patterns (Sokol et al., 1998)

\begin{tabular}{|c|c|c|c|c|}
\hline Pattern & $\begin{array}{c}\text { Number of } \\
\text { Columns }\end{array}$ & $\begin{array}{c}\text { Connection } \\
\text { Strength (kN) }\end{array}$ & $\begin{array}{c}\text { Connection } \\
\text { Strength per } \\
\text { Screw (kN) }\end{array}$ & Group Effect \\
\hline 4C & 1 & 6.64 & 1.66 & 0.71 \\
\hline 4A & 2 & 6.70 & 1.68 & 0.72 \\
\hline 4B & 3 & 6.94 & 1.74 & 0.74 \\
\hline 4E & 2 & 7.04 & 1.76 & 0.75 \\
\hline 4D & 4 & 7.40 & 1.85 & 0.79 \\
\hline
\end{tabular}

Note: $0.76 \mathrm{~mm}$ thick steel, No. 8 screw, 3d spacing 
According to Sokol et al. (1998), and as seen by the "Group Effect" given in Table 2-6, varying the screw pattern did not significantly vary the strength of the connection and the "Group Effect" values were within $\pm 7 \%$ of the average. With the exception of pattern $4 \mathrm{E}$, a general trend was however observed where an increase in the number of columns resulted in an increase in connection strength. This trend was attributed to the fact that the additional columns provided enhanced rotational stability to the connection. By way of explanation, the loading on the connection is eccentric due to the fact that the sheets overlap each other, which causes the connection to rotate. An increase in columns thus results in a connection which is more stable against rotation where less rotation translates into increased strength. When the connection rotates, tilting of the screws occur, and instead of being in shear, the screws experience tension and shear. This leads to screws that tend to pull out of the steel rather than bear on them, and in turn decreases the capacity of the connection.

Moreover, Sokol et al. (1998) noted that this trend occurred repeatedly. When observing the behaviour of connections consisting of only two screws, patterns given in Figure 2-21, it can be seen that pattern $2 \mathrm{~A}$ consisted of a single row perpendicular to the applied load and pattern 2B comprised of two columns parallel to the applied load. The connection strengths, as well as the calculated group effect, are given in Table 2-7, where it can be seen that the 2B pattern provided more resistance to rotation which in turn developed improved structural performance. Similar results were obtained for other sheet steel thicknesses and size screws.

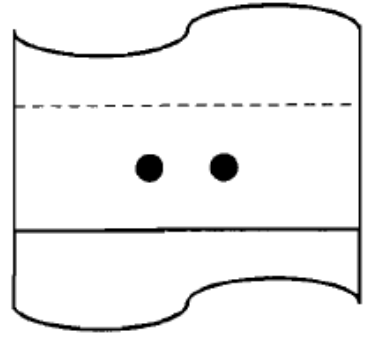

2A

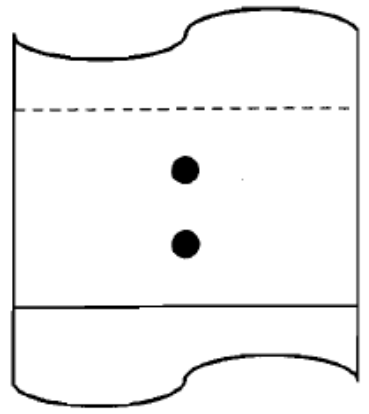

2B

Figure 2-21 Screw patterns for two screws (Sokol et al., 1998)

Table 2-7 Effect of Number of Columns on Connection Strength (Sokol et al., 1998)

\begin{tabular}{|c|c|c|c|c|}
\hline Pattern & $\begin{array}{c}\text { Number of } \\
\text { Columns }\end{array}$ & $\begin{array}{c}\text { Connection } \\
\text { Strength (kN) }\end{array}$ & $\begin{array}{c}\text { Connection } \\
\text { Strength per } \\
\text { Screw (kN) }\end{array}$ & Group Effect \\
\hline 2A & 1 & 5.10 & 2.55 & 0.83 \\
\hline 2A & 1 & 5.33 & 2.67 & 0.87 \\
\hline 2B & 2 & 5.29 & 2.64 & 0.86 \\
\hline 2B & 2 & 5.70 & 2.85 & 0.93 \\
\hline
\end{tabular}

Note: $1.02 \mathrm{~mm}$ thick steel, No. 8 screw, 3d spacing 
Sokol et al.; (1998) further found that another pattern dependent behaviour which occurred, was the failure of a connection in which the screw pattern did not take up a significant proportion of the sheet width. An example of this occurrence is depicted in Figure 2-22 where patterns 10D and 12C do not take up as much width as compared to pattern 10A and 12A. Both of these examples involved fracture, not bearing failures, although Sokol et al. (1998) noted that the same behaviour was observed for bearing failures where connections consisted of eight screws. Data comparing these patterns, along with the calculated group effect, are given in Table 2-8.

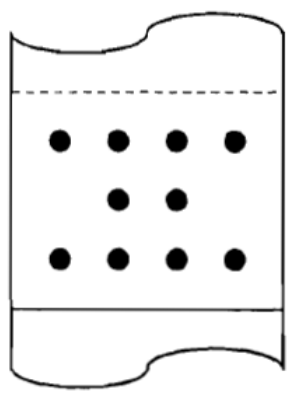

$10 \mathrm{~A}$

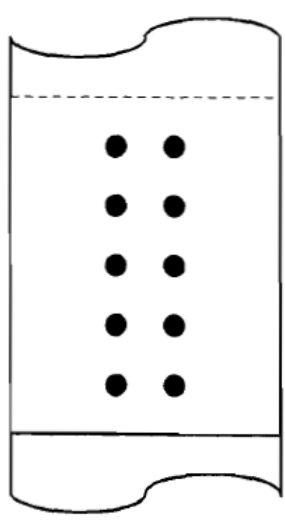

$10 \mathrm{D}$
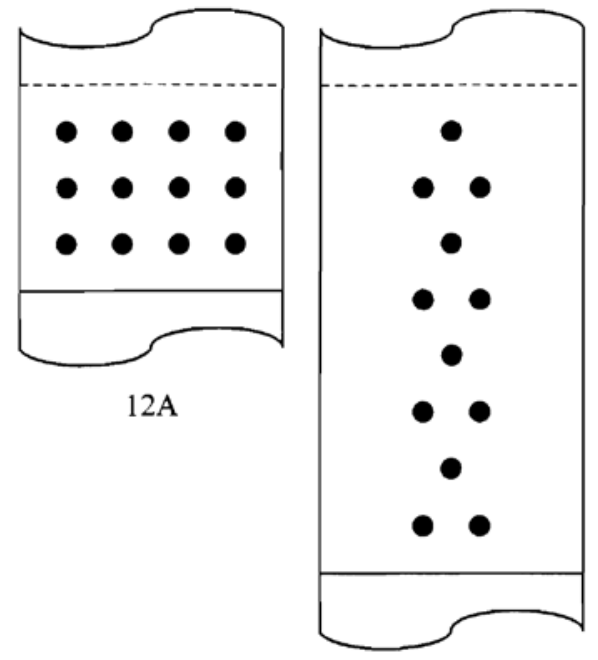

$12 \mathrm{C}$

Figure 2-22 Screw patterns for ten and twelve screws (Sokol et al., 1998)

Table 2-8 Effect of Cupping (Sokol et al., 1998)

\begin{tabular}{|c|c|c|c|}
\hline Pattern & $\begin{array}{c}\text { Number of } \\
\text { Columns }\end{array}$ & $\begin{array}{c}\text { Connection } \\
\text { Strength (kN) }\end{array}$ & $\begin{array}{c}\text { Connection } \\
\text { Strength per Screw } \\
\mathbf{( k N )}\end{array}$ \\
\hline $10 \mathrm{~A}$ & 3 & 14.81 & 1.48 \\
\hline $10 \mathrm{D}$ & 5 & 13.64 & 1.36 \\
\hline $12 \mathrm{~A}$ & 3 & 16.76 & 1.40 \\
\hline $12 \mathrm{C}$ & 8 & 11.95 & 1.00 \\
\hline
\end{tabular}

Note: $0.76 \mathrm{~mm}$ thick steel, No. 8 screws for $10 \mathrm{~A}$ and 10D, No. 12 screws for $12 \mathrm{~A}$ and 12C, 3d spacing

Based on the generality developed above, one would expect patterns 10D and 12C to provide enhanced structural capacity due to the higher number of columns present in the connection. However, considering the fact that the patterns for 10D and 12C were narrow with respect to the sheet width, Sokol et al. (1998) stated that the sheet "cupped” under the load. This was described as the occurrence where the edges of the sheet curled around the screw pattern, so that both sheets curled away from each other (Figure 2-23). The researchers attributed this phenomenon to the sheet stretching more in the vicinity of the screws and less at the edges of the sheet. 


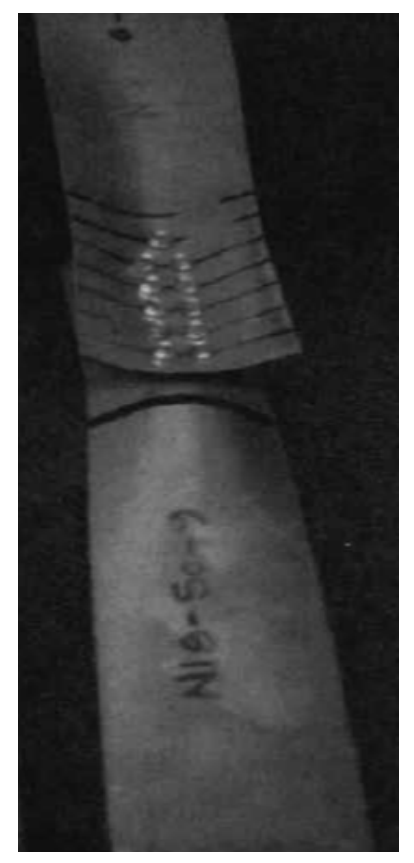

Figure 2-23 Failure Due to Cupping of the Steel (Sokol et al., 1998)

\subsection{FINITE ELEMENT ANALYSIS OF LAP SHEAR CONNECTIONS}

Considering the exponential increases in computers' processing power and data handling abilities, combined with the rapid development of engineering software, numerical simulation is becoming ubiquitous in almost all fields of research. Numerical analysis has become an incredibly powerful tool in the field of structural engineering, and together with experimental analysis can provide insight into factors such as stresses and strains, which are not easily determined from experimental testing alone. Once numerical simulation has been calibrated against physical experimental tests, a large number of similar simulations can be performed with different parameters, eschewing the requirement for further physical testing, providing a highly economical solution.

\subsubsection{Chung \& Ip $(2000 ; 2001)$}

Chung \& Ip (2000; 2001) created finite element models of bolted connections between coldformed steel strips and hot-rolled steel plates under static shear loading. The load-extension curves were predicted through the use of the software ANSYS. Their recommended procedure for creating an FE model was through detailed study of the stress-strain curves, the clamping force developed in the bolt shank, the frictional coefficient between the element interfaces, and the contact stiffness. The load-deflection curves deduced from the numerical analysis compared well to the obtained test results. 
Their proposed model was calibrated against 12 lap shear tests, utilising both G300 and G550 cold-formed steel strips, each of 1.5 and $2.5 \mathrm{~mm}$. In all the tests, two identical cold-formed steel strips were bolted to a $25 \mathrm{~mm}$ hot-rolled steel plate at one of their ends, as shown in Figure 2-24. The extension rate was set to $1 \mathrm{~mm} / \mathrm{min}$, up to a maximum total end extension of $5.0 \mathrm{~mm}$, whilst data was collected in $0.05 \mathrm{~mm}$ intervals.

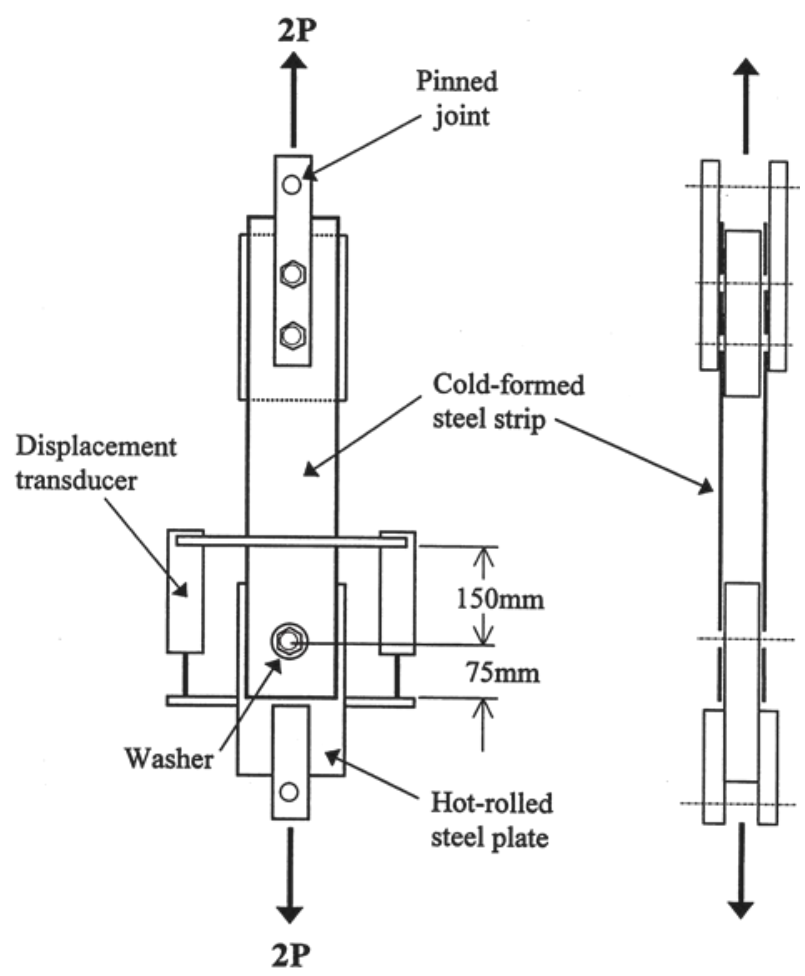

Figure 2-24 Configuration of typical test specimen in lap shear test (Chung \& Ip, 2000; 2001)

All of the components were modelled using three-dimensional, eight-node iso-parametric SOLID45 solid elements, to effectively capture yielding propagation through the material, including; the cold-formed steel strips, the hot-rolled steel plates, as well as the bolt and the washers. These elements were found to be appropriate for this plasticity type problem, since "discontinuous strain fields were allowed in simulating shear bands" (see Appendix A). Furthermore, the normal stresses acting upon the cold-formed steel strips, due to clamping forces in the bolt shanks; and the tangential stresses, as a result of frictional forces between contact interfaces; can also be incorporated. CONTAC49 contact elements were utilised to model the contact surfaces, eschewing the requirement for intuitive assumptions regarding the size and position of the contact area.

For the sake of simplicity, only half of the lap shear test was modelled, as depicted in Figure 2-25, through symmetrical division of the test specimen along the longitudinal axis. The hotrolled steel plate was modelled using a single element, and was assumed to be rigid and fixed in space, with all degrees of freedom restrained. The bolt formed an integral component with the washer, and was assumed to be both threadless, and to remain linear elastic throughout the analysis. The root of the bolt-washer component was fixed in space through restriction of the associated nodes. 


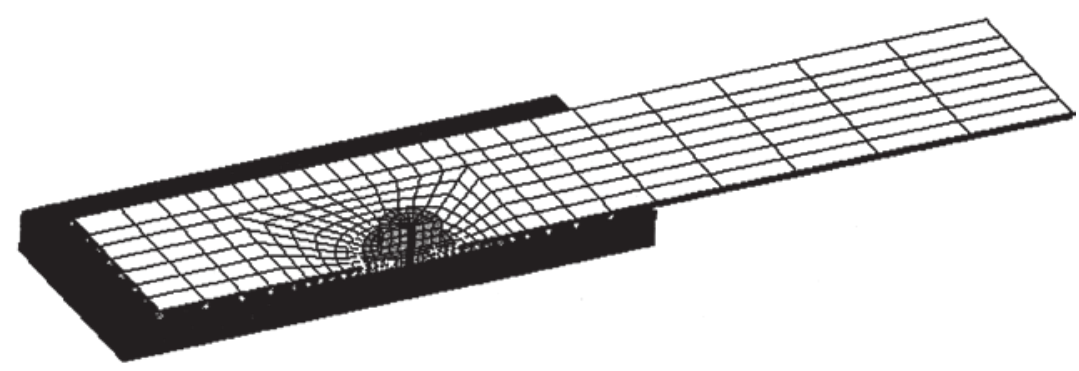

Figure 2-25 Overall view of connection (Chung \& Ip, 2000; 2001)

In order to simplify meshing, a small artificial hole, $1 \mathrm{~mm}$ in diameter, was provided through the centreline of the bolt-washer component. As depicted in Figure 2-26, the finite element mesh in the vicinity of the bolt hole was refined for the purposes of improved resolution of stresses and deformations; allowing local buckling in the strips to also be incorporated. In order to reduce the shear locking (see Appendix A) effect in the elements, the aspect ratios of the elements in proximity to the bolt hole were kept to a minimum. This was noted to be particularly important for elements on the contact surface, due to the requirement that they conform to the shape of the bolt-shank when simulating the bolt-hole interaction.

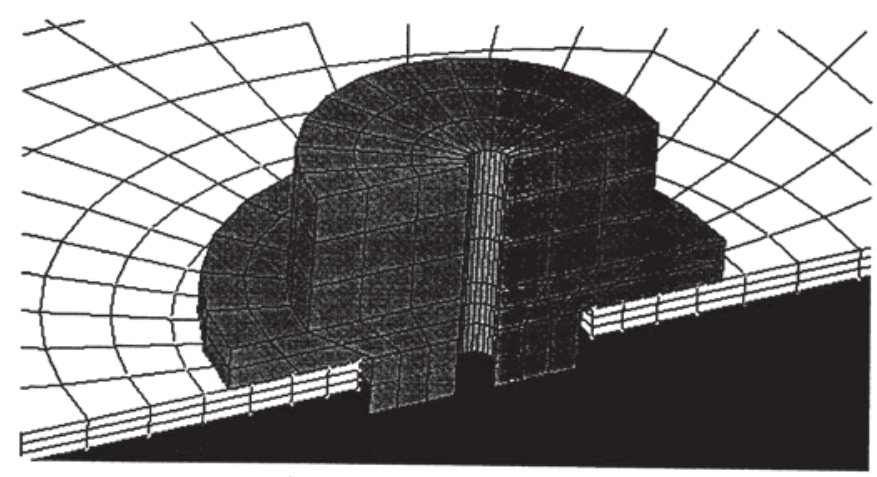

Figure 2-26 Enlarged view of bolt and washer (Chung \& Ip, 2000; 2001)

Coupon tests were used to establish the "true" stress-strain curves, and was adopted for the cold-formed steel strips, allowing the yielding and strength degradation during deformation to be determined. The results of Chung \& Ip's (2000; 2001) coupon tests yielded a bi-linear stress-strain curve, which was incorporated into the finite element model; where the yield strength was taken as the measured yield strength, $\mathrm{p}_{\mathrm{y}}$, of the cold-formed steel strips. Chung \& Ip (2000; 2001) referred to the finite element model with such a material curve as FEA- $p_{y}$.

It is possible to model local fractures in steel materials; through the use of extremely small elements with "birth and death" (see Appendix A) characteristics; though the computational effort and complexity of the finite element mesh will be considerably increased. Thus, a simplified method was used, assuming the steel strength to reduce linearly, from the maximum value to zero, after the measured ductility limit is reached, that is, the strength degradation. 
Thus, strength degradation was proposed as an engineered stress-strain curve, with reduced strength at high strain after yielding. Whilst the ultimate tensile strength and corresponding strain are based on tensile coupon tests, these curves were assumed to be valid for the same material under compression. A negative slope was added to the curve after the ultimate compressive strength was attained, to account for the effect of strength degradation under sizable compressive forces. The value of the negative slope in the strength degradation portion, was selected as double the post-yielding tangent modulus of the steel. The compressive strength was then reduced linearly to zero at a strain of around $50 \%$, and the finite element model with this material curve was referred to as FEA-p $\mathrm{p}_{\text {. }}$

A third bi-linear stress-strain curve was proposed, where the measured tensile stress, $U_{s}$, served as the basis for the yield strength, as determined from data obtained through coupon tests. The researchers referred to the finite element model utilising this material curve as FEA-Us. The three proposed stress-strain curves obtained by Chung \& Ip (2000; 2001); namely FEA- $p_{y}, F E A-p_{r}$ and FEA- $U_{s}$; were assumed to be rate independent, and are presented in Figure 2-27. It was noted that, when compared to a similar approach in a finite element modelling of local fracture, as reported by Fan et al. (1997b), the proposed stressstrain curves were fairly conservative.

(a)

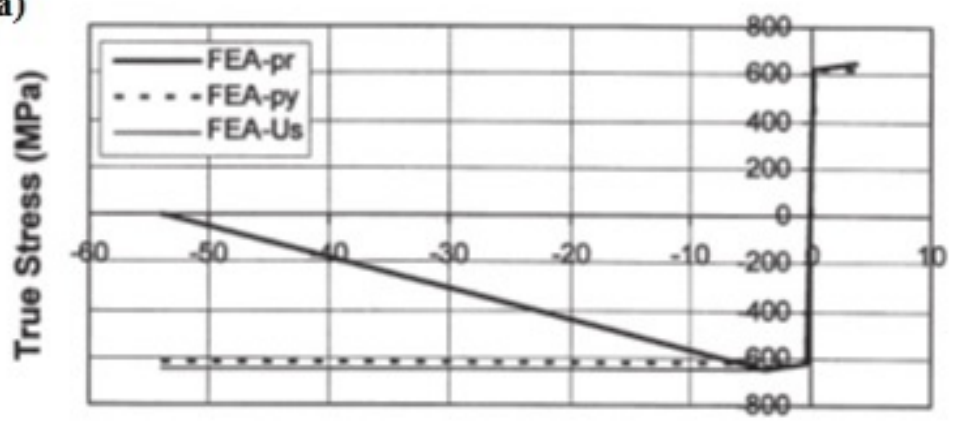

True Strain (\%)

(b)

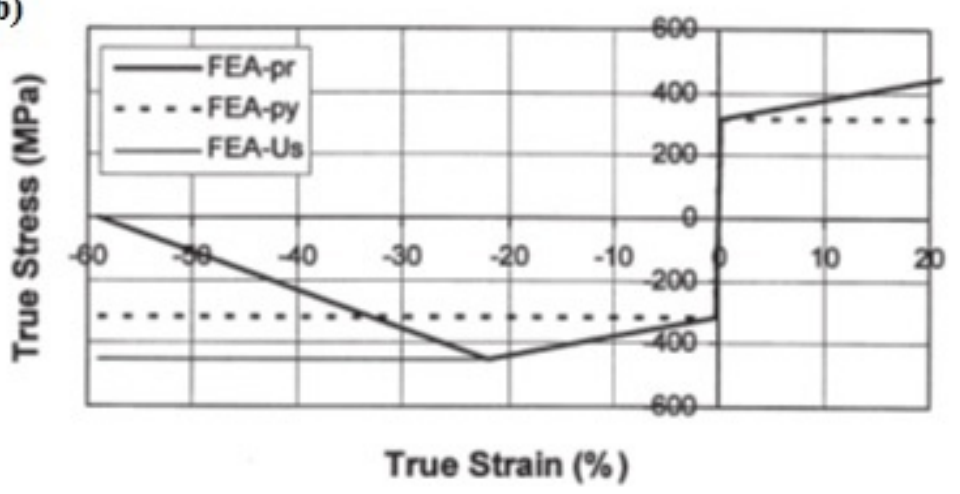

Figure 2-27 Proposed stress-strain curves (a) G550 cold-formed steel strips under tension and compression. (b) G300 cold-formed steel strips under tension and compression (Chung \& Ip, 2000; 2001) 
Utilisation of the penalty method (Appendix A); in which the contact element is tetrahedral in shape, defined by a contact node on one surface, with four other nodes on the target surface; was required in order to effectively simulate the required contact conditions. Prevention of over penetration of the associated target surfaces by the contact nodes, through the development of contact forces acting on the nodes; comprising normal and tangential components in relation to the target surface; ensures compatibility between the interfaces. The nodes around the bolt holes, associated with the cold-formed steel strips, were chosen as the contact nodes, with the facets of the shank acting as the target surfaces. In order to ensure convergence, the contact stiffness was selected to be $2000 \mathrm{~N} / \mathrm{mm}$, with a friction coefficient of 0.2 used for all contact surfaces; both of these values were assumed to remain constant throughout the analysis.

In order to incorporate the material, geometrical and contact non-linearities, Chung \& Ip (2000; 2001) utilised a non-linear analysis. Through the use of a displacement controlled method, the total load was applied in a series of small increments, up to a maximum extension of $3 \mathrm{~mm}$. The maximum resistance of the bolted connections was also assumed to be at $3 \mathrm{~mm}$, thus preventing excessive deformation of the connections. The maximum plastic strain increment after each incremental load was recommended as $5 \%$, though the average displacement increment was found to be $0.25 \mathrm{~mm}$, which corresponded to a $3.5 \%$ incremental plastic strain. At this strain level, there were significant changes in the shape and orientation of the individual elements, and it was noted that their nodal displacements and stiffness affected each other considerably. The problem was noted to be highly non-linear, necessitating the employment of the full Newton-Raphson procedure; with all out-of-balance forces, nodal displacements and tangent stiffness matrix of the structure being updated with each equilibrium iteration. A force-based convergence criterion was selected, requiring the square root of the sum of squares, SRSS, of the load imbalance, to be less than $1 \%$ of the SRSS of the applied loads in the equilibrium iteration.

The finite element model allowed Chung \& Ip (2000; 2001) to gain information with regards to the stress distribution in the strips experiencing end bearing failure, the resistance contributions of the bearing and frictional actions, as well as the yield zones of the coldformed steel strips. A schematic of the typically deformed shape of the bolted connection is depicted in Figure 2-28. It was found that the FEA- $\mathrm{p}_{\mathrm{r}}$ finite element model was recommended for the high-strength, low ductility G550 cold-formed steel strips, as a result of the other models, FEA- $\mathrm{U}_{\mathrm{s}}$ and FEA-py, typically providing a $15 \%$ higher resistance at $3 \mathrm{~mm}$. Notably, the FEA-py model gave very conservative results for the high ductility G300 samples, due to the low yield strength values, with the FEA- $\mathrm{U}_{\mathrm{s}}$ model producing much better results, as a result of the higher yield strength. It should be noted that variation in local deformation and diversified strain levels within the bolted connections were not at all considered in the model. The FEA-pr model was recommended once again, as it allowed for strength degradation at high strain values. 


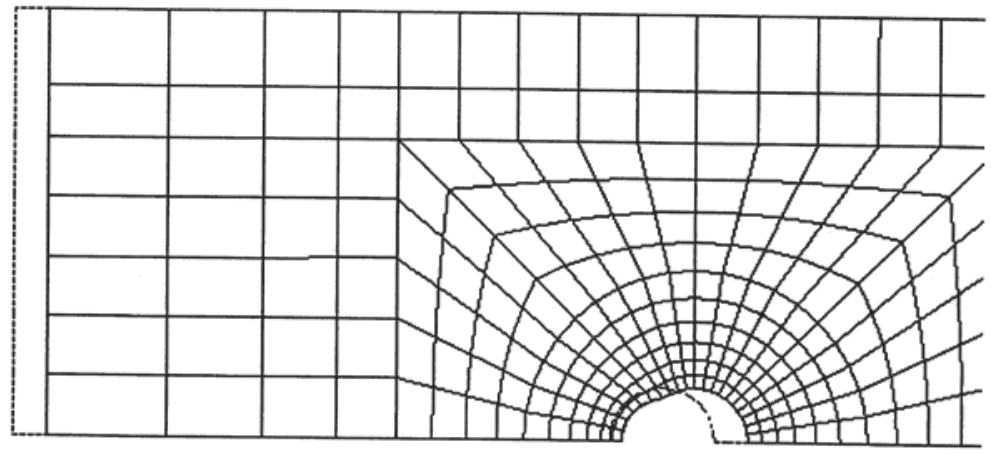

Figure 2-28 Deformed shape at 3 mm extension (Chung \& Ip, 2000; 2001)

The von Mises (see Appendix A) stress distribution of the FEA-py and FEA-pr finite element models, for a specimen at $3 \mathrm{~mm}$ extension, is shown in Figure 2-29. Chung \& Ip (2000; 2001) noted that the strength degradation in the FEA-pr model is evident as a result of the large local strains in the elements directly contacting the bolt shank, even though the yield zones in both models are similarly sized, leading to a $15 \%$ reduction in load carrying capacity of the connection when compared with the FEA-py model. Additionally, interactions between the yield zones of the individual bolts may occur when the bolts are closely spaced, further reducing the load carrying capacity of the connection.
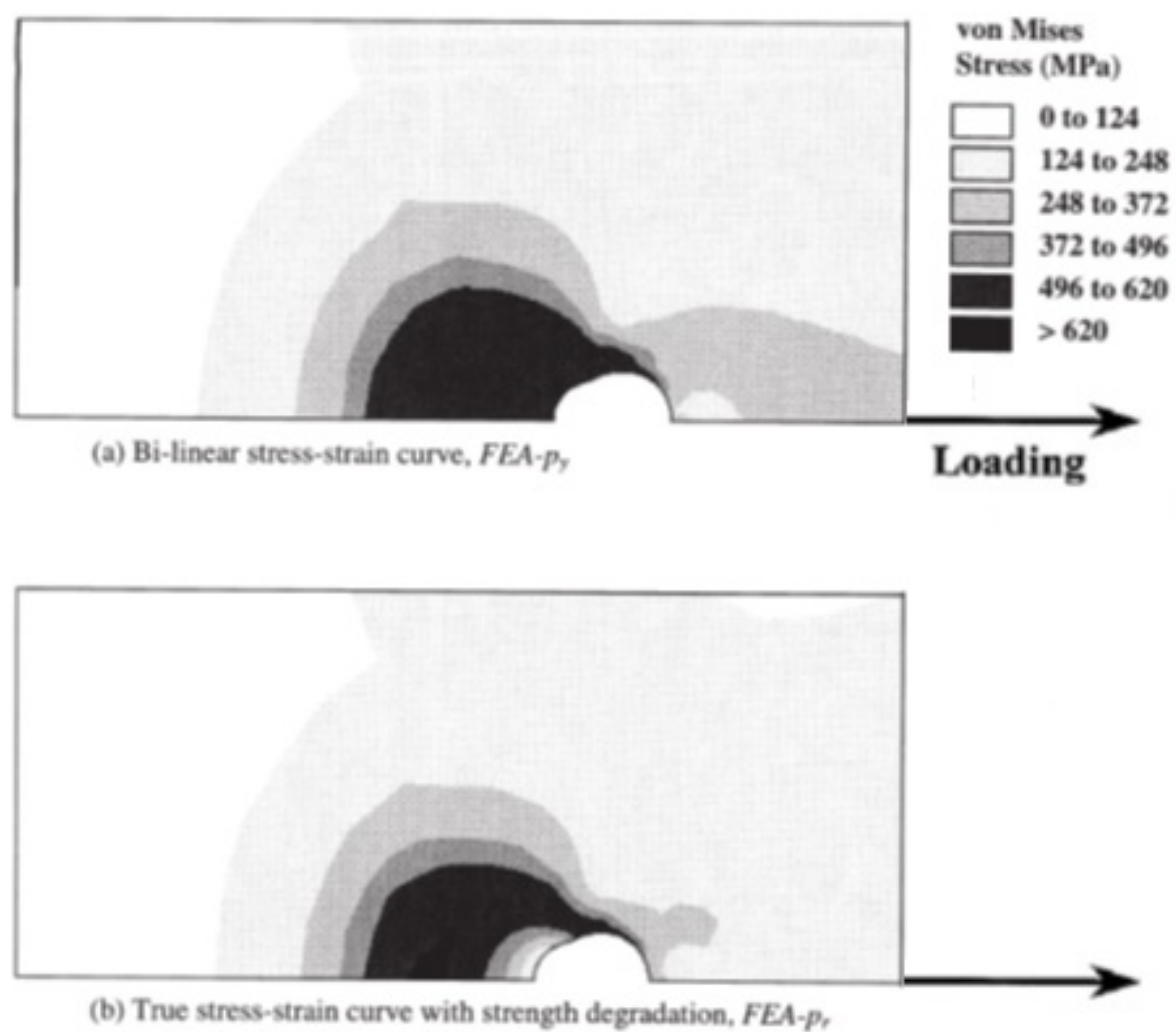

Figure 2-29 Stress distribution of bolted connection G550 specimen at $3 \mathrm{~mm}$ extension (Chung \& Ip, 2000; 2001) 
Chung \& Ip (2000; 2001) determined that the finite element model could effectively predict the bearing failure of the cold-formed steel strips in the bolted connections. The von Mises stresses in close proximity of the bolt holes, across the width of the steel strips for both high and low strength steels at different extensions $(\delta)$, are shown in Figure 2-30. During the course of load application, there are significant variations in both the magnitude and shape of stress distributions, due to the large local deformations. The researchers also demonstrated that the bearing resistance of bolted connections are adversely affected by any reduction in the ductility of the steels.

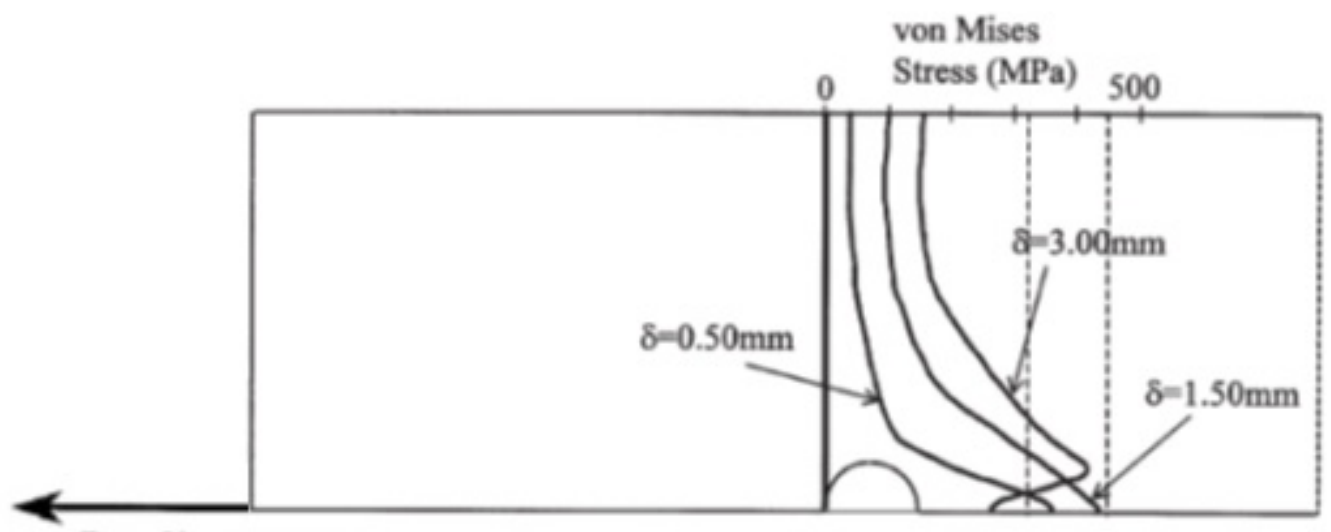

\section{Loading (a) $\mathrm{t}=1.50 \mathrm{~mm}, \mathrm{G} 300$}

von Mises

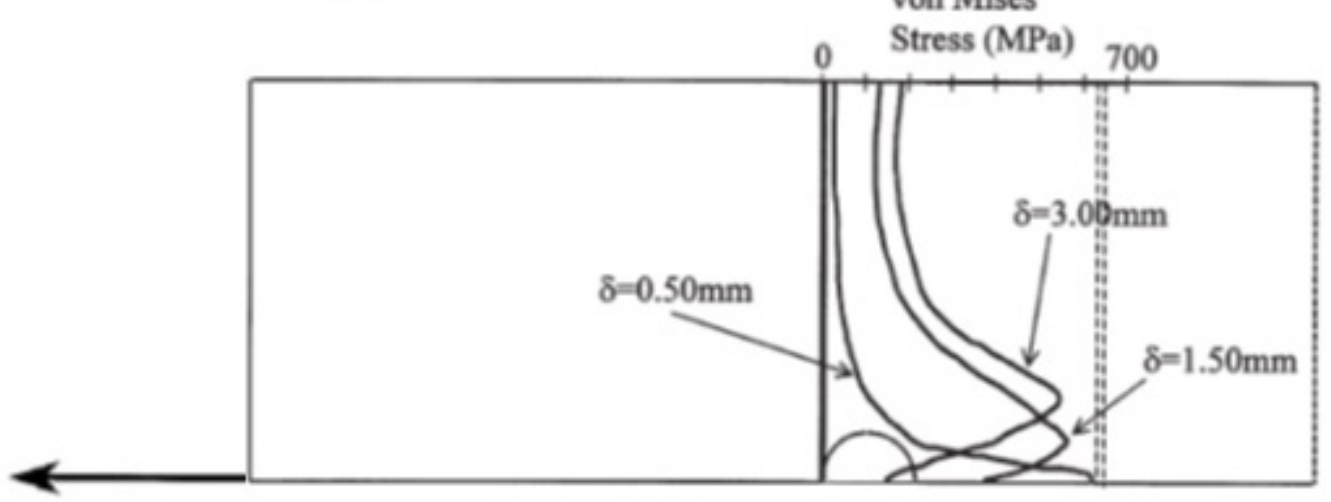

(b) $t=1.60 \mathrm{~mm}$, G550

Figure 2-30 Von Mises stress distribution of bolted connection (Chung \& Ip, 2000; 2001)

The von Mises stress distribution around the bolt holes of the G550 test specimens are presented at three different extensions, in Figure 2-31. The elements directly contacting the bolt shank around the bolt hole reveal a high stress concentration, typically several times the applied stress, as a result of direct end bearing. The yield zones rapidly increase in size as extension increases, due to the yielding of these elements. Chung \& Ip (2000; 2001) determined that the stresses of these elements were only one third of the yield strength of the cold-formed steel strips at an extension of $3 \mathrm{~mm}$, as illustrated by the large strain variations in the vicinity of these elements, in Figure 2-32. The maximum direct strain, $\varepsilon_{\mathrm{x}}$, was predicted to be $40 \%$, confirming large plastic flow of materials surrounding the bolt hole, in direct contact with the bolt shank. 

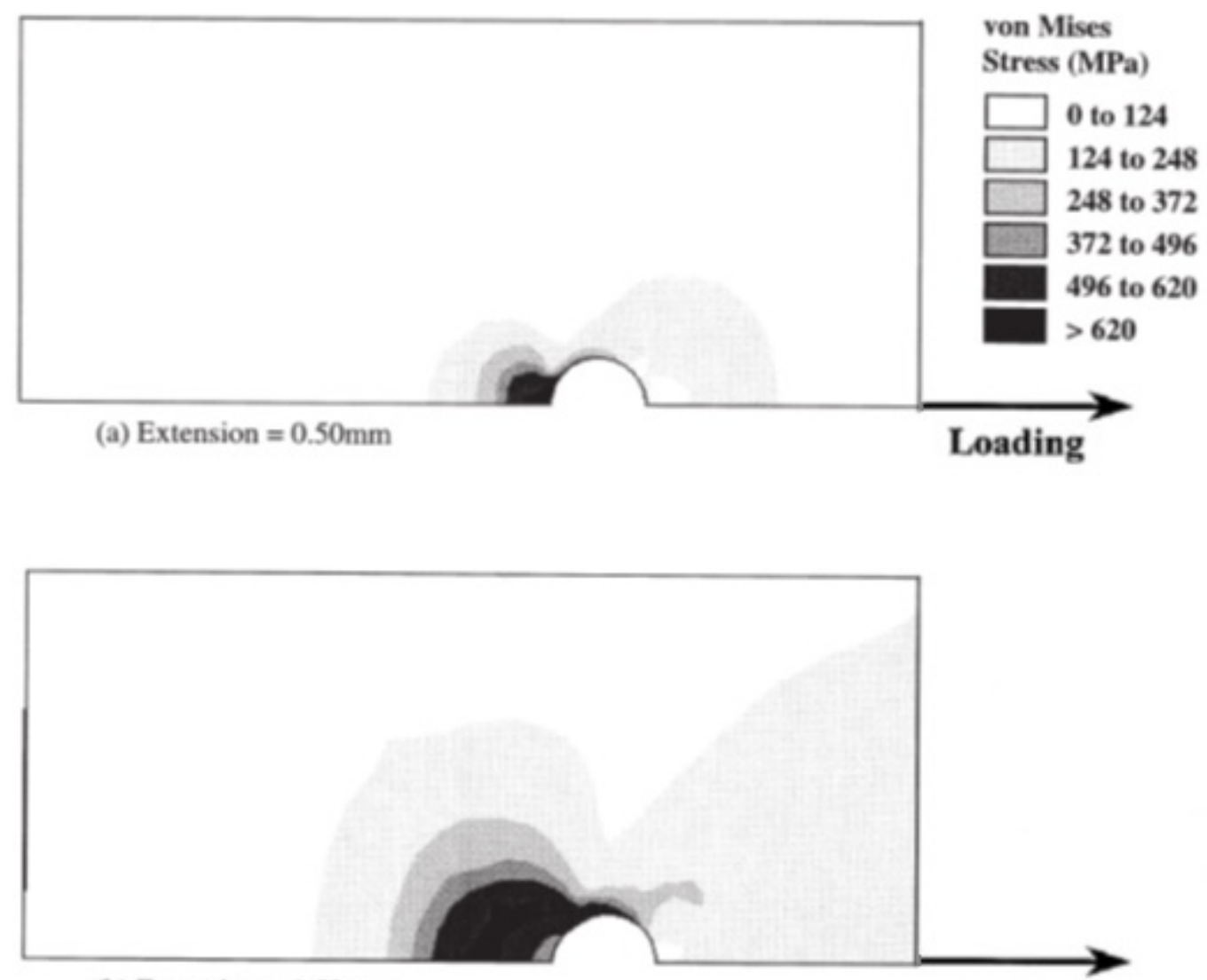

(b) Extension $=1.50 \mathrm{~mm}$

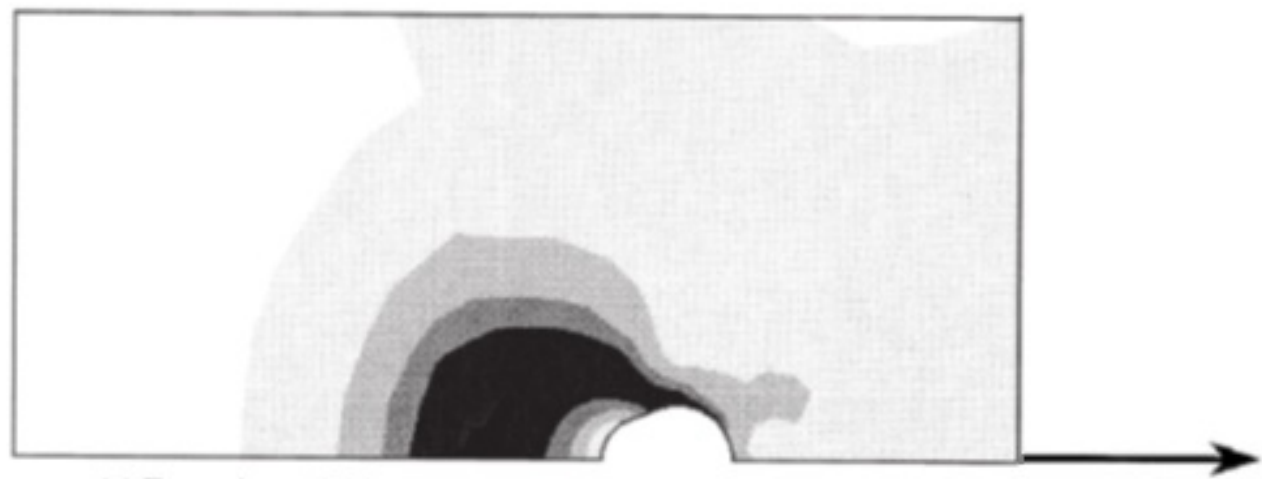

(c) Extension $=3.00 \mathrm{~mm}$

Figure 2-31 Stress distribution of bolted connection G550 specimen with extension of (a) 0.50 mm; (b) 1.50 mm; (c) 3.00 mm (Chung \& Ip, 2000; 2001) 

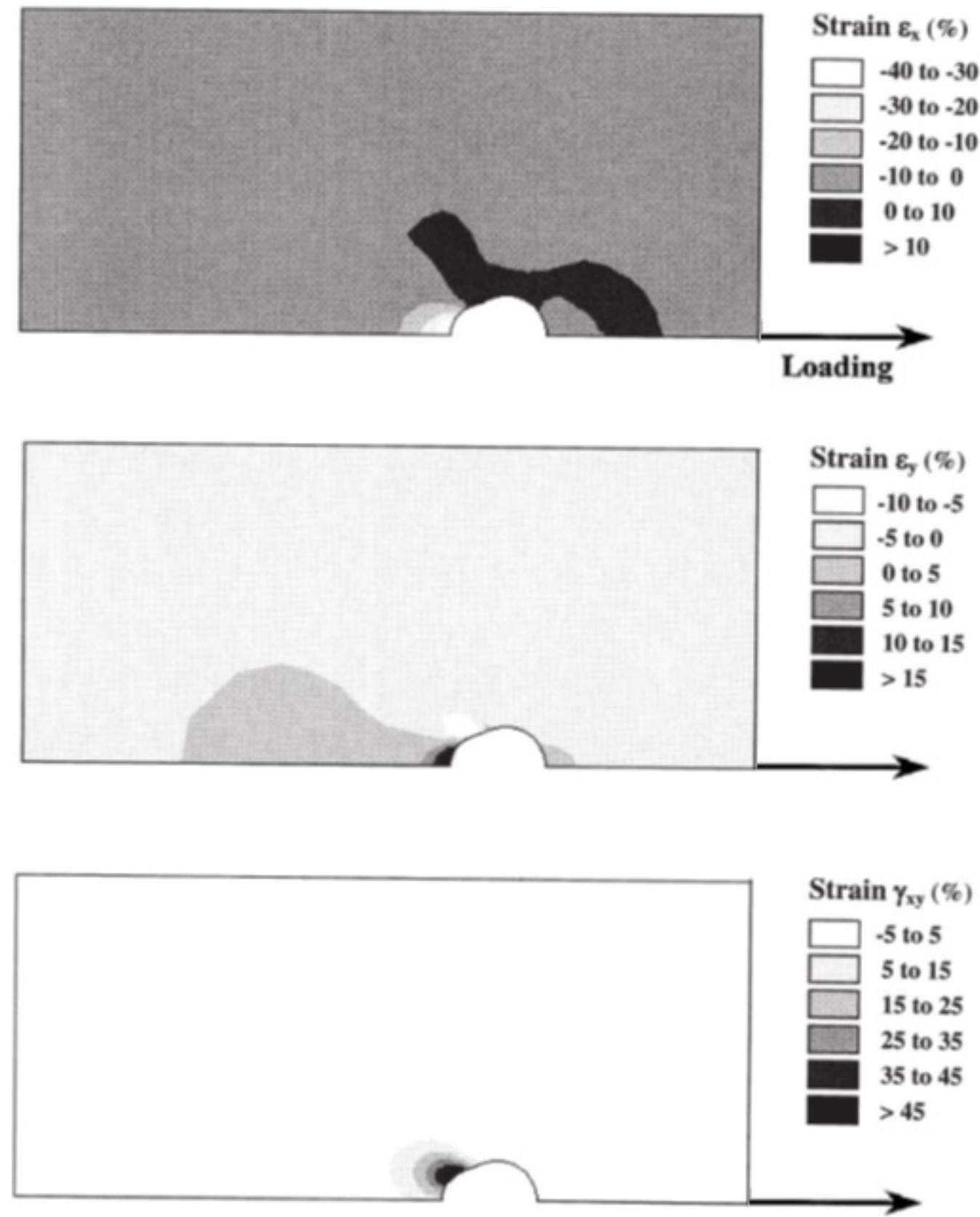

Figure 2-32 Strain components of bolted connection G550 specimen at $3 \mathbf{~ m m}$ extension (Chung \& Ip, 2000; 2001)

For the G300 and G550 bolted connections, with various thicknesses, the von Mises stress distributions were normalised to the yield strength, as shown in Figure 2-33. When compared to the G300 test specimens at an extension of $3 \mathrm{~mm}$, yielding of the G550 specimens were confined to a small area surrounding the bolt hole. Chung \& Ip (2000; 2001) concluded that and that the yield patterns differ according to the ductility limits of the steel, and that strength degradation was less significant for high ductility steels. It was further noted that the yield patterns were similar for bolted connections utilising different thicknesses of the same grade steel. 

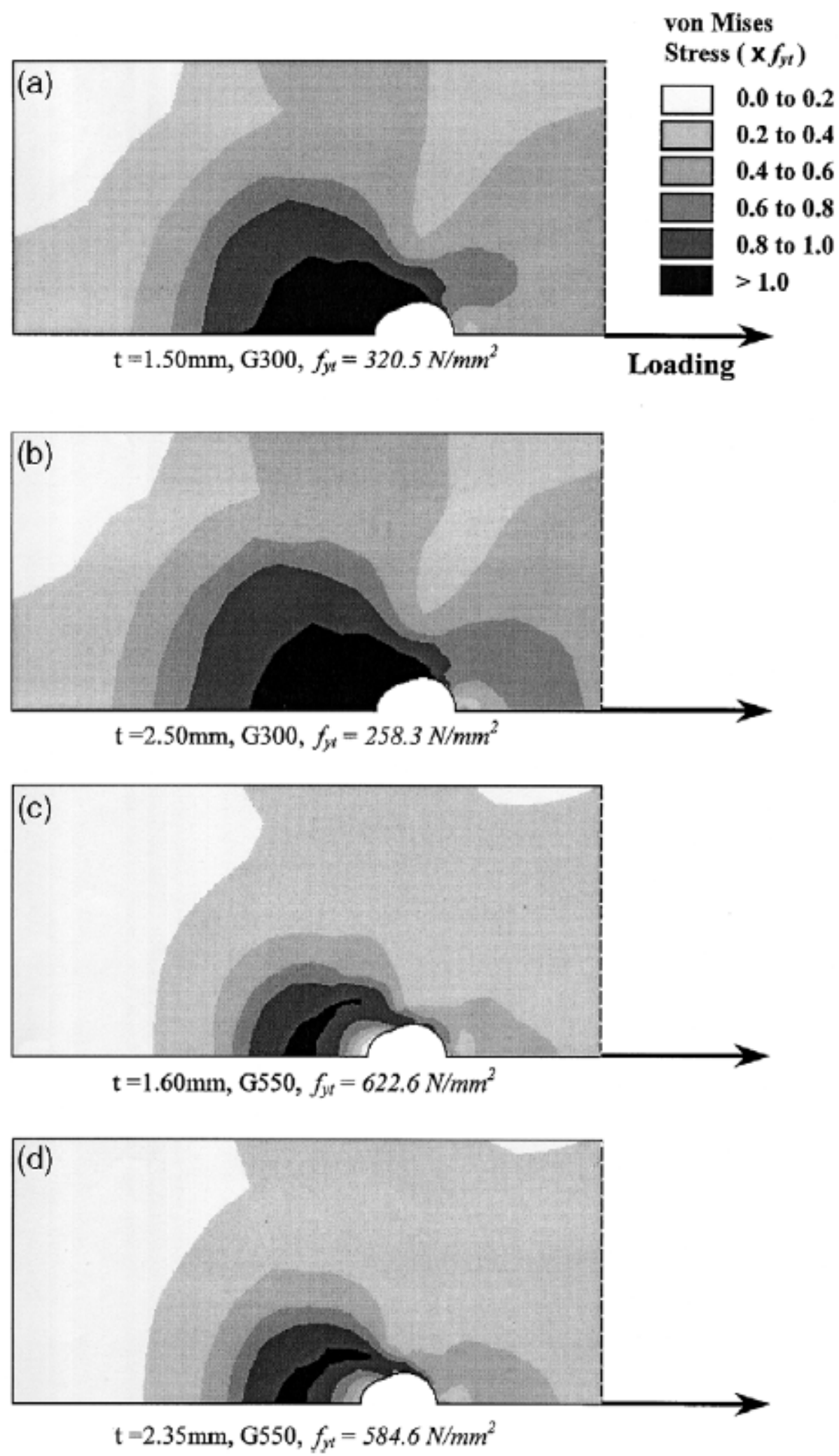

Figure 2-33 Stress distribution of bolted connections at $3 \mathrm{~mm}$ extension (Chung \& Ip, 2000; 2001) 


\subsubsection{Kim \& Kuwamura (2007)}

Kim \& Kuwamura (2007) used ABAQUS to establish a finite element model, with threedimensional solid elements, calibrating the model against existing data. They furthermore carried out parametric studies to investigate the structural behaviour of bolted connections utilising thin-walled stainless steel plates. The researchers quantitatively examined the effect of curling on the ultimate strength, proposing suitable failure criteria to predict the mode of failure of bolted connections.

The objective of their FE analysis was to identify the simplest model which would provide a sufficiently accurate description of reality. The specimens comprised 1.5 and $3.0 \mathrm{~mm}$ thick stainless steel plates, with yield strengths of 235 and $440 \mathrm{~N} / \mathrm{mm}^{2}$ respectively, connected to $6.0 \mathrm{~mm}$ thick plates, utilising 12 and $15 \mathrm{~mm}$ diameter bolts tested in single shear. The model took symmetry into consideration, by only modelling half of the specimens, and only modelled the 1.5 and $3.0 \mathrm{~mm}$ plates, considering the $6.0 \mathrm{~mm}$ plate to be rigid and undeformable under loading.

The bolts in the model were assumed to be snug-tightened, threadless and cylindrically shaped. Considering this was a bearing type connection, no bolt-pretension or rotation was simulated, and in the vicinity of the hole, the plates resisted the applied force directly. The influence of the loading position was also considered, and the conclusion was made that the ultimate behaviour of bolted connections in this model was unaffected by any difference in the loading position. The model, along with the various boundary conditions, is shown in Figure 2-34.

The main reason for the analysis was the prediction of the ultimate strength and failure mode of thin-walled bolted connections, accompanying the local instabilities and curling of the plate. Kim \& Kuwamura (2007) used two types of elements; namely solid and shell elements; to model the plates, and concluded that solid elements were much more suited to demonstrate the ultimate behaviour of bolted connections for thin-walled steel.

Curling is defined as the out-of-plane deformation in the plate thickness direction and can be seen in Figure 2-35. Curling is particularly likely to occur in thin-walled steel bolted connections as the end distance increases. Local buckling of the plate in the compressive stress field of the bearing part with the bolt is also believed to cause curling. Kim \& Kuwamura (2007) found that, when compared with FE models which were restrained from curling, the ultimate strength was reduced by $4-25 \%$ in FE models in which curling occurred. They also found that should curling occur after the ultimate strength is attained, that the effect of curling was negligible. 
(a)

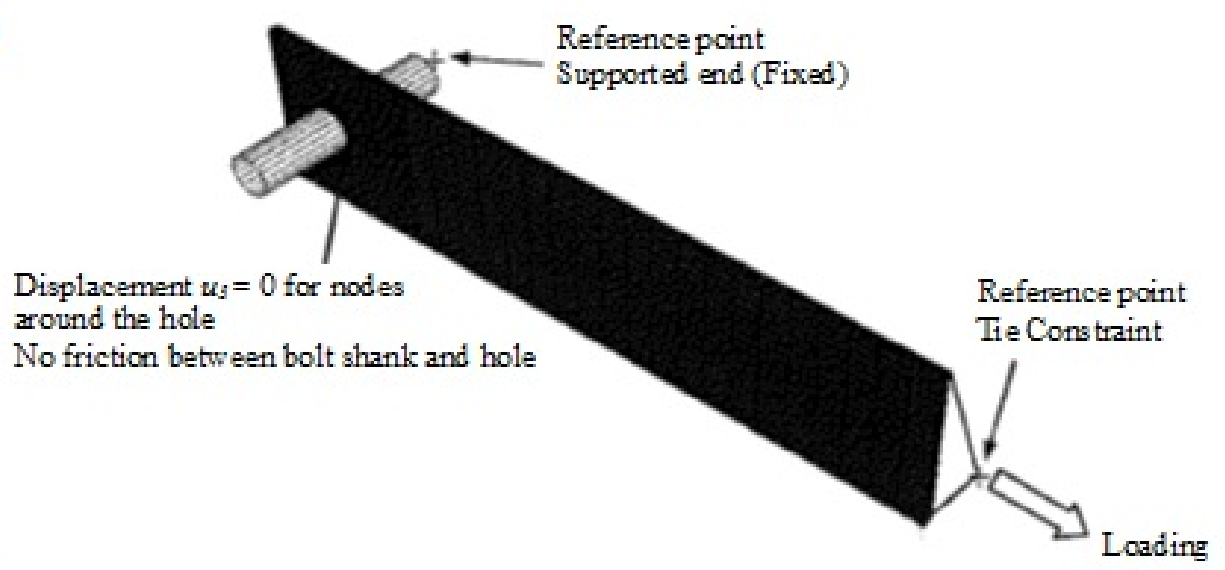

(b)

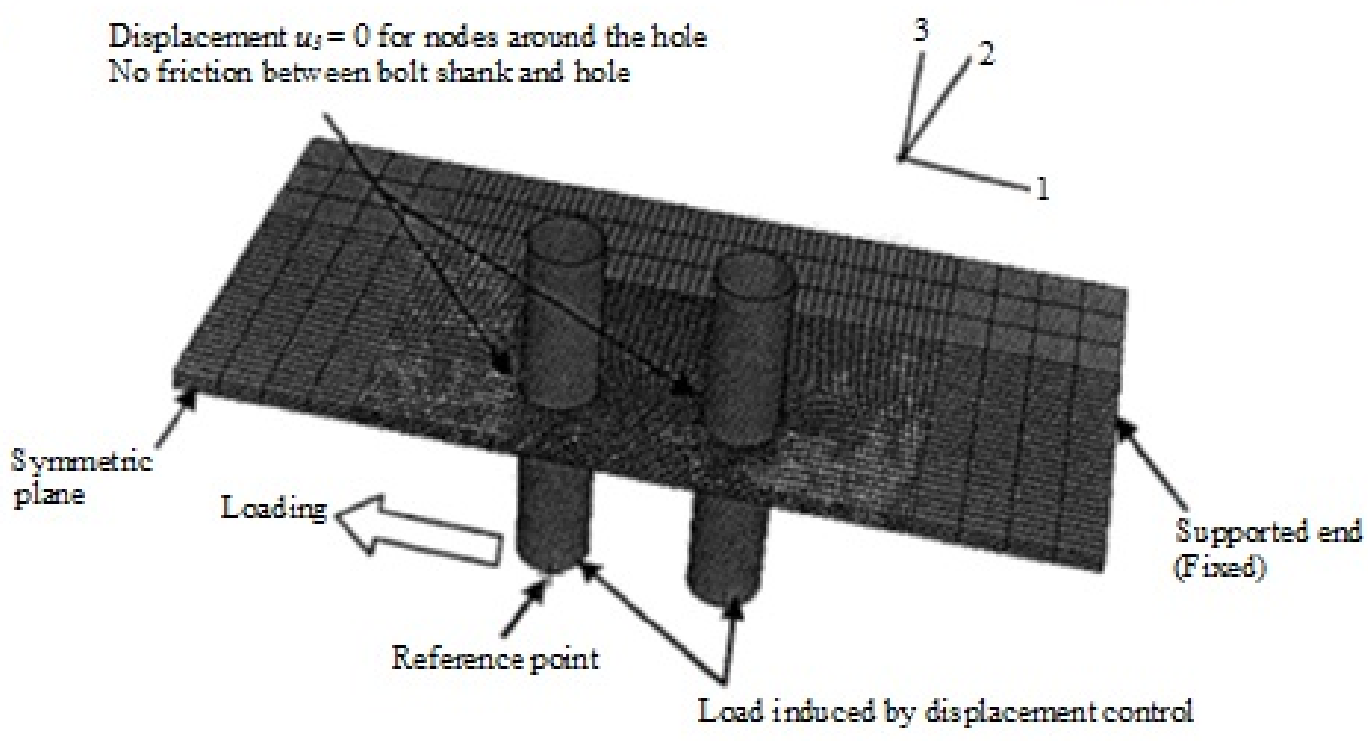

Figure 2-34 Details of finite element models (a) loading applied at reference point; (b) loading applied at bolts (Kim \& Kuwamura, 2007)

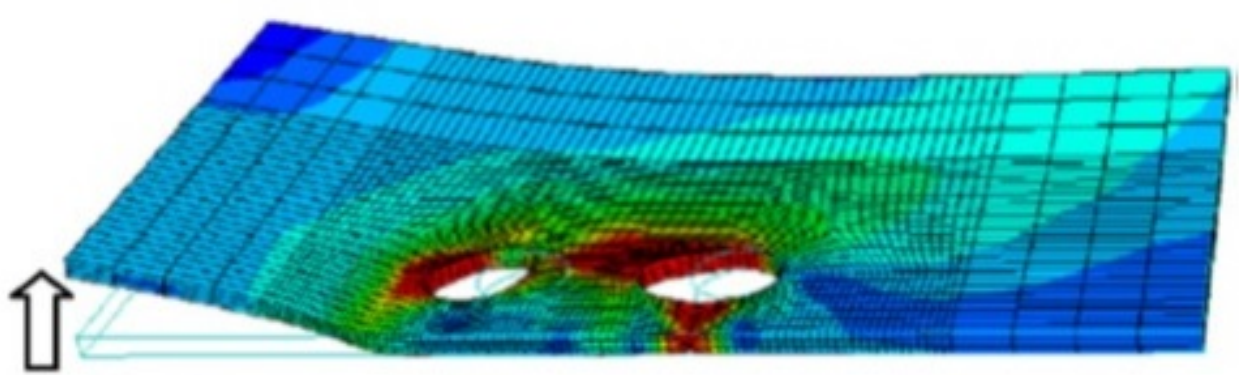

\section{Curling}

Figure 2-35 Deformed shape showing curling (Kim et al., 2008) 


\subsubsection{Kim, Kuwamura \& Cho (2008)}

Kim et al. (2008) performed a parametric study on the ultimate strength of four bolted connections in single shear with curling, which failed by block shear failure. They proposed a revised design formula considering the effect of curling on bolted connections, through use of correlations between the strength reduction ratio and the plate thickness. Through comparison with existing FE analysis results and test results, the proposed design equations predicting the ultimate strength were validated.

To perform their parametric study, Kim et al. (2008) used the model proposed by Kim \& Kuwamura (2007), which is shown in Figure 2-36. The main geometric parameters that were varied included:

- End distance $\quad$ - $\quad$ Ranging from $1.0 \mathrm{~d}$ to $5.0 \mathrm{~d}$

- Edge distance - $\quad$ Ranging from $1.0 \mathrm{~d}$ to $5.0 \mathrm{~d}$

- Plate thickness $\quad$ - $\quad 1.5,3.0$ and $6.0 \mathrm{~mm}$

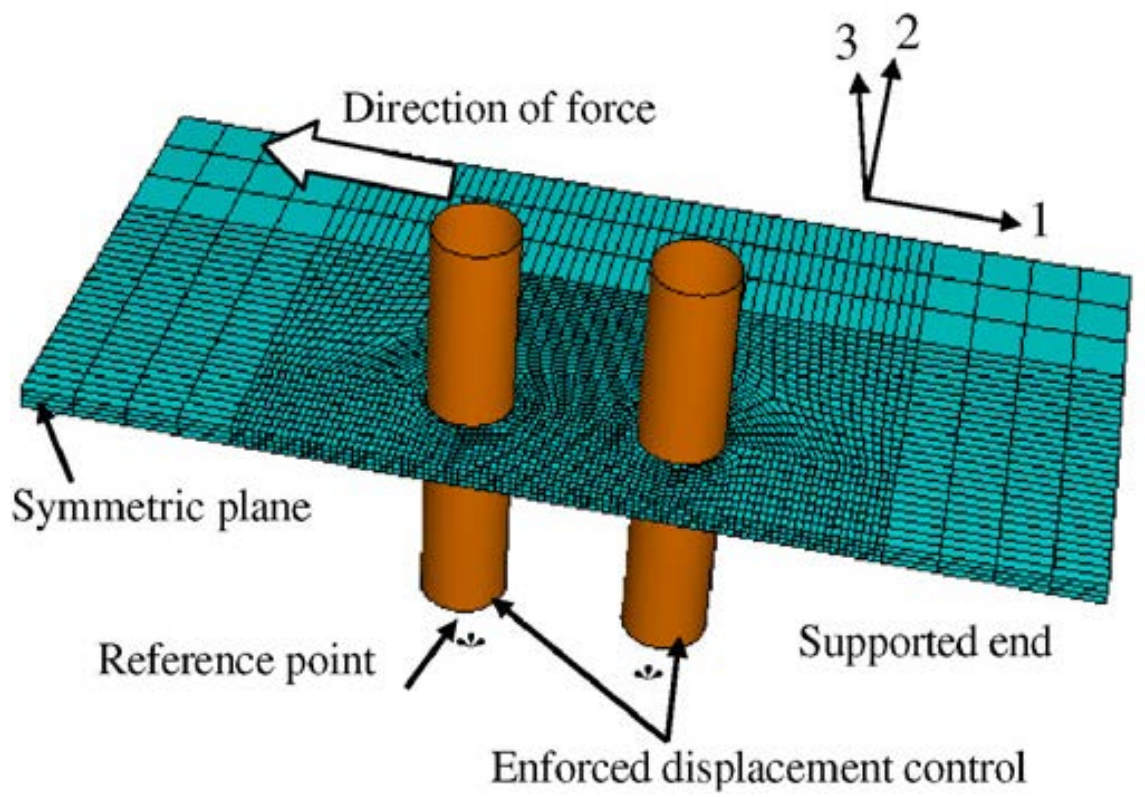

Figure 2-36 FE model with boundary conditions (Kim et al., 2008)

Kim et al. (2008) discovered that the manifestation of curling varied according to plate thickness, as shown in Figure 2-37. It was shown that as the plate thickness decreased, curling became more prevalent. 
(a)

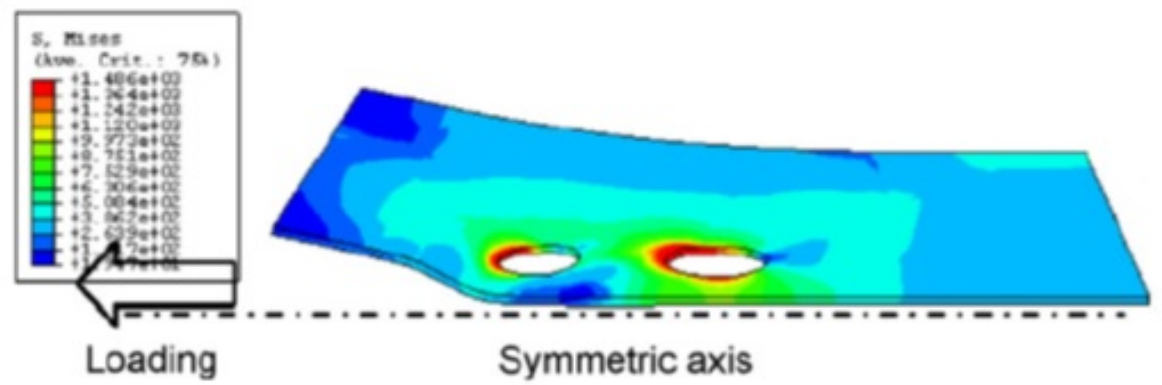

(b)

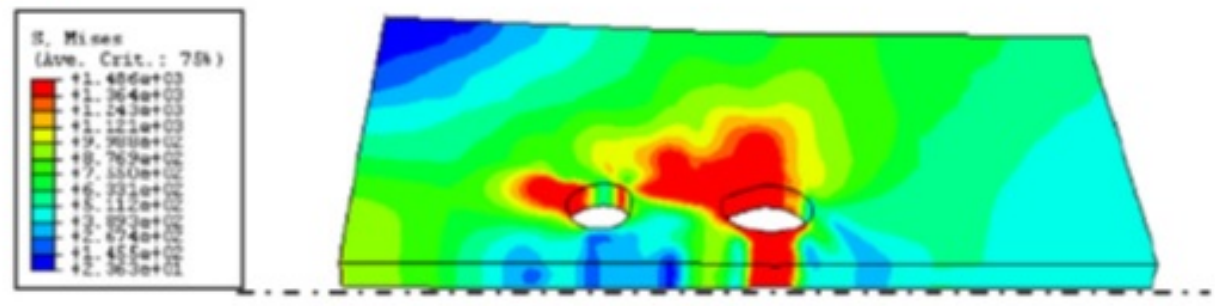

Symmetric axis

Figure 2-37 Difference of curling behaviour according to plate thickness (a) $1.5 \mathrm{~mm}$ plate thickness; (b) $6.0 \mathrm{~mm}$ plate thickness (Kim et al., 2008)

\subsubsection{Salih, Gardner \& Nethercot (2010)}

Salih et al. (2010) performed a numerical investigation examining the behaviour of stainless steel double lap connections which failed through net section rupture. The numerical models for ferritic and austenitic stainless steel, of 8 and $10 \mathrm{~mm}$ thicknesses respectively, were developed and validated against existing test results. The results indicated that, prior to fracture, the ductility of stainless steel was sufficient to ensure extensive redistribution of stresses. The researchers proposed a revised design equation for the net section capacity of stainless steel connections.

The stress-strain behaviour of stainless steel differs from that of carbon steel, as depicted in Figure 2-38. It was noted that using the conventional elastic-perfectly plastic material model, commonly used for carbon steel, to represent stainless steel behaviour in FE models lead to inaccurate results. The following compound Romberg-Osgood stress-strain expressions were employed in the FE models, in order to describe the non-linear behaviour of stainless steel.

$$
\varepsilon=\left\{\begin{array}{lr}
\frac{\sigma}{E}+0.002\left(\frac{\sigma}{\sigma_{0.2}}\right)^{n} & \text { for } \sigma \leq \sigma_{0.2} \\
\frac{\sigma-\sigma_{0.2}}{E_{0.2}}+\varepsilon_{u}\left(\frac{\sigma-\sigma_{0.2}}{\sigma_{u}-\sigma_{0.2}}\right)^{m}+\varepsilon_{0.2} & \text { for } \sigma_{0.2} \leq \sigma \leq \sigma_{u}
\end{array}\right.
$$


Where $\varepsilon$ is the nominal strain, $\sigma$ is the corresponding nominal stress, $E$ is the initial Young's modulus, $E_{0.2}$ is the tangent stiffness at the $0.2 \%$ proof stress, $\sigma_{0.2}$ is the $0.2 \%$ proof stress, $\varepsilon_{0.2}$ is the total strain at the $0.2 \%$ proof stress, $\varepsilon_{u}$ is the strain at the ultimate stress $f_{u}$ and $n$ and $m$ are the strain hardening coefficients.

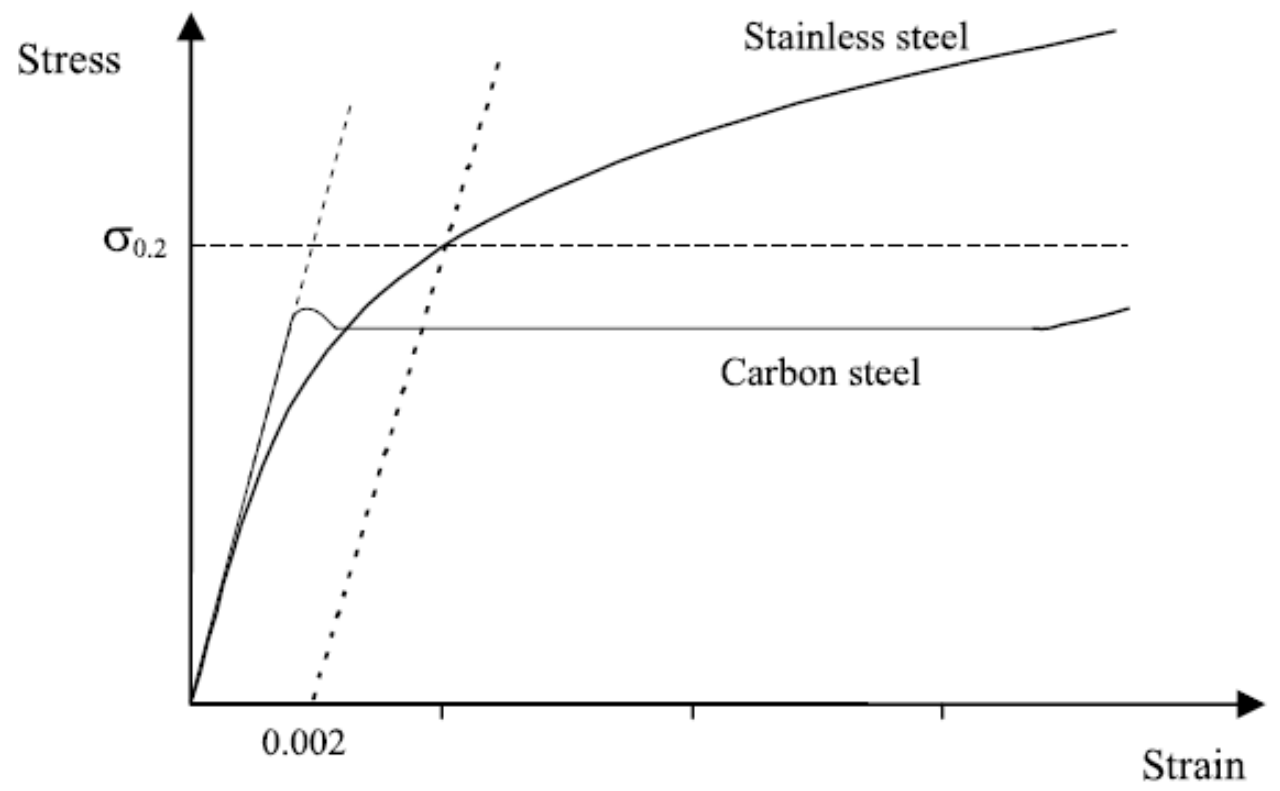

Figure 2-38 Stress-strain curves for carbon and stainless steel (Salih et al., 2010)

It is expected that FE analysis for bolted connections would involve large inelastic strains, thus the nominal stresses and strains were converted to the corresponding true stresses $\left(\sigma_{\text {true }}\right)$ and true plastic strains $\left(\varepsilon_{\text {true }}^{p l}\right)$. The true stress in a stress-strain curve continues to rise beyond the nominal ultimate stress until the occurrence of fracture. In order to model the full material response until fracture so as to capture the localised stresses and strains, the true stress-strain curve was extended beyond the true ultimate stress up to a fracture strain point of $100 \%$ using the equation:

$$
\sigma_{\text {true }}=K\left(\varepsilon_{\text {true }}^{p l}\right)^{p} \quad \text { for } \sigma_{u, t r u e}<\sigma_{\text {true }} \leq \sigma_{\text {frac,true }}
$$

Where $\sigma_{u, t r u e}$ is the true ultimate stress, $\sigma_{\text {frac,true }}$ is the true fracture stress, $K$ is the strength constant and $p$ is the strain hardening exponent. A three-dimensional eight-node linear solid element was employed. Contact between all components expected to interact with each other was defined through the use of contact pairs, using surface-to-surface contact with a friction coefficient of 0.2. A general static analysis with displacement control loading was utilised, incorporating the Newton-Raphson method of obtaining solutions for the non-linear problem. 
Due to the algorithm employed to solve such problems, models containing contact definitions were noted to be computationally expensive. An inspection of the state of all contact interactions is performed at the beginning of each increment, in order to determine whether the slave nodes are open or closed. A further check is performed if the node is closed, to identify whether the node is sticking or sliding. The algorithm applies constraints to each closed node, and releases them from each open node, repeating this process until no change can be found in the status of any slave nodes. Thereafter, an equilibrium iteration is performed, neglecting the increment if the residuals are not within the prescribed tolerance, and instead repeating the procedure with a smaller increment. Salih et al. 2010 stated that attaining convergence in such models can be extremely difficult, and often before an entire solution can be found, the program is terminated due to numerical instability.

Salih et al. (2010) noted that ductility played an important role in the net section failure of the connections, and as a result of stress concentration, local yielding at the hole edge would allow for stress redistribution at the net section, resulting in necking and finally a ductile failure. Conversely, in non-ductile materials, the stress concentration surrounding to the hole would not be redistributed, causing immediate cracking and resulting in a brittle failure. Figure 2-39 exhibits the typical results for bearing and net section failure.
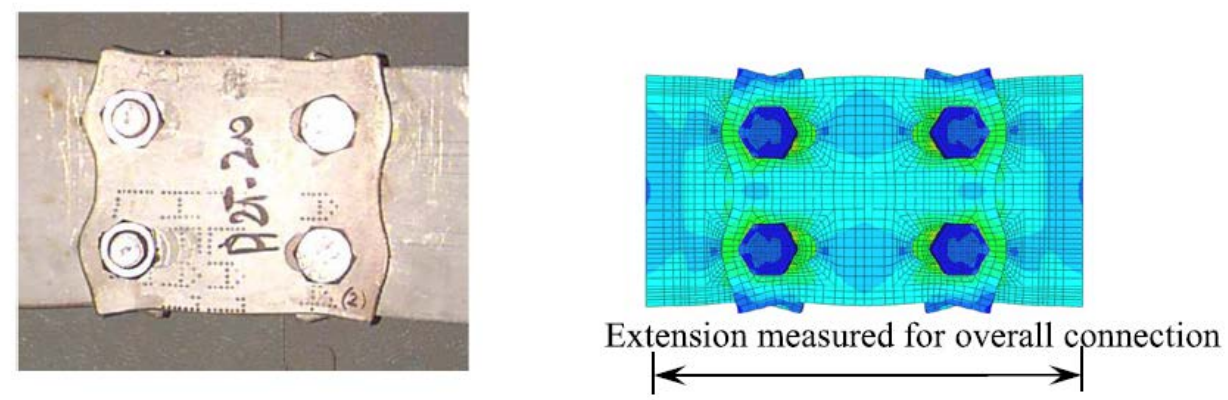

(a) Bearing failure (type I connection).

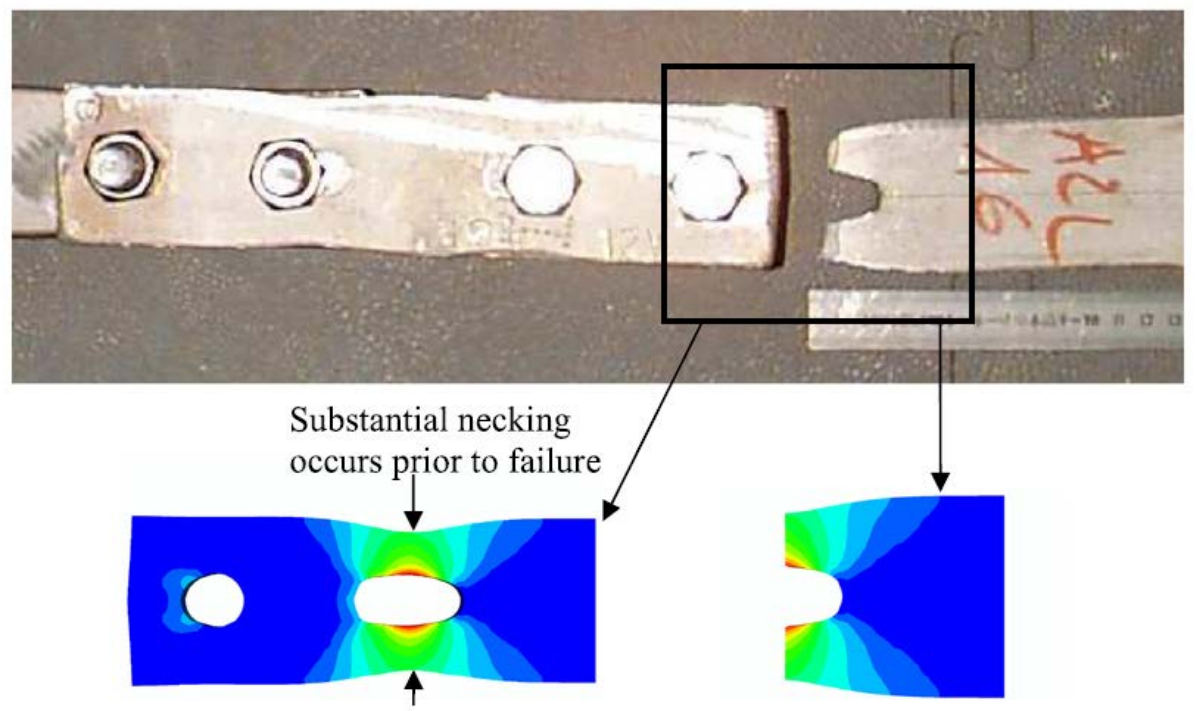

(b) Net section failure (type II connections).

Figure 2-39 Comparison between deformed test specimens and numerical model (Salih et al., 2010) 


\subsubsection{Moze \& Beg (2011)}

In order to obtain the distribution of forces between bolts, Moze and Beg (2011) numerically simulated double shear bolted connections, fabricated from high strength steel. The researchers conducted a total of 26 tests, along with numerically investigating several tests gathered from literature regarding similar connections made of high strength steel. As the numerical results correlated well with the experimental results, they were used to evaluate the bearing resistance formula in accordance to EN 1993-1-8 (2005).

The FE package ABAQUS was used to simulate the tests, and all components were modelled as deformable bodies through the use of three-dimensional eight-node elements, with contact interactions described between the parts. A "hard" surface-to-surface contact formulation was defined in the normal direction, both between the bolt head and the plate, as well as the bolt shank and the bolt hole in the plate. The penalty friction formulation (Appendix A), defined only through a coefficient of friction equalling 0.25 , was described between the specimen and the cover plates.

Several simplifications were introduced in the modelling of the connection assembly, in order to shorten the total computational time. Elastic material properties were prescribed to the cover plates and bolts, as they were designed to deform elastically. Additionally, whilst the bolts could deform elastically along their axis, due to pressure of the cover plates on the bolt heads, the displacement between the bolts remained constant. This meant that there was no requirement to define the contact between the bolt shank and the cover plates, as the whole bearing force was transmitted to these supports. A coarser mesh was used to model the cover plates, as their only function was to introduce frictional forces into the connection and prevent deformation in the thickness of the specimen. A typical FE model used in their study is shown in Figure 2-40. 


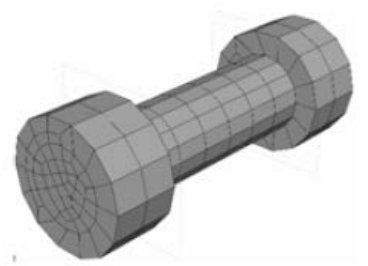

(a) Deformable, elastic bolt.

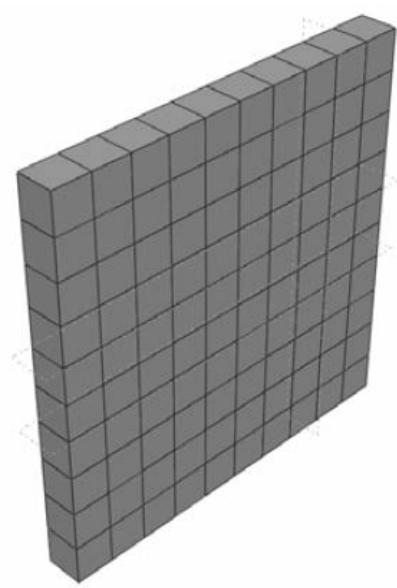

(c) Deformable, elastic cover plate.

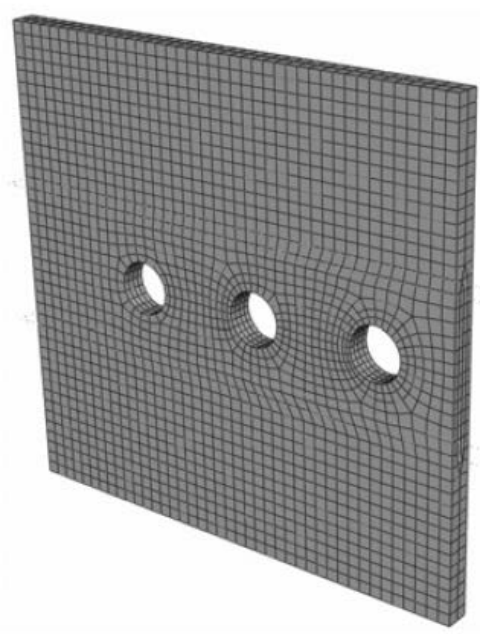

(b) Deformable inner plate.

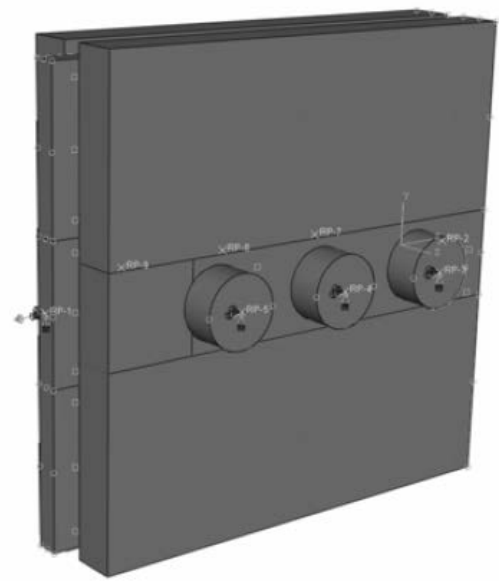

(d) The connection.

Figure 2-40 Numerical model of connection (Moze \& Beg, 2011)

The structural steel was modelled as an elastic-plastic material, where the plasticity was obtained from standard tensile tests, as a polygonal approximation of the stress-strain curve. The engineering material curves were translated into the form of logarithmic strain and true stress for the purposes of the FE model. Young's modulus was taken as E = $210 \mathrm{GPa}$ with a Poisson's ratio of 0.3 . The experimental and numerically obtained load-displacement curves of the standard tensile tests are shown in Figure 2-41. 


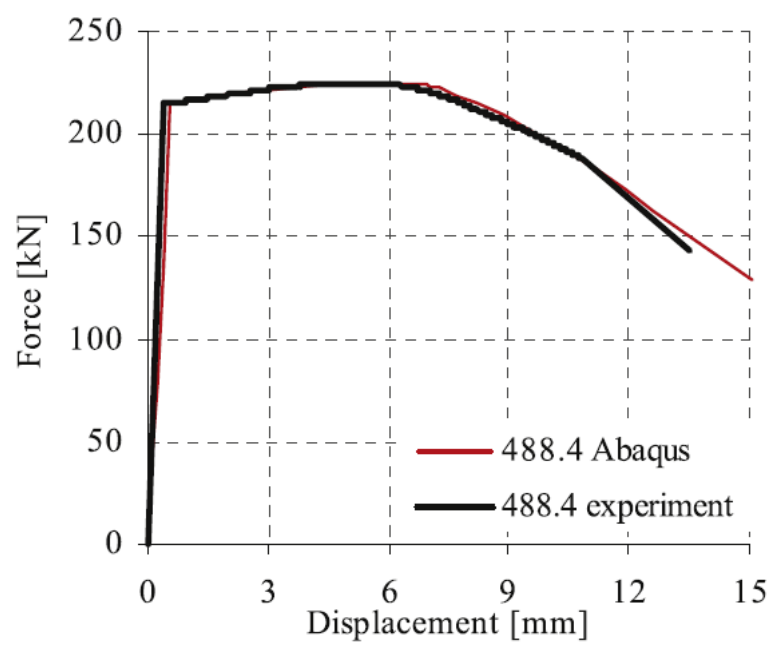

Figure 2-41 Comparison of numerical and experimental load-displacement curves of standard tensile test (Moze \& Beg, 2011)

For all kinds of failures, the numerical responses correlated well with the experimental results, in both resistance as well as deformation state, as depicted in Figure 2-42. The objective of the study was to establish whether sufficient local ductility could be assured, in order to transfer the loading between all bolts, in bolted connections comprised of highstrength steel. Numerical simulation in conjunction with testing indicated that, even when the position of the bolts was unfavourable, high strength steel connections possessed sufficient ductility to redistribute the loading.

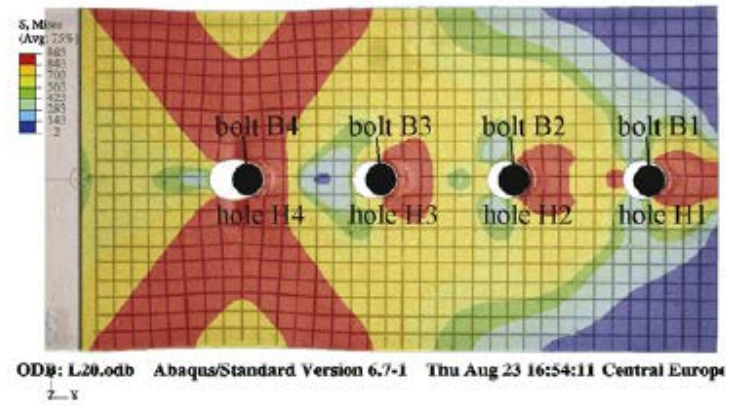

(a)

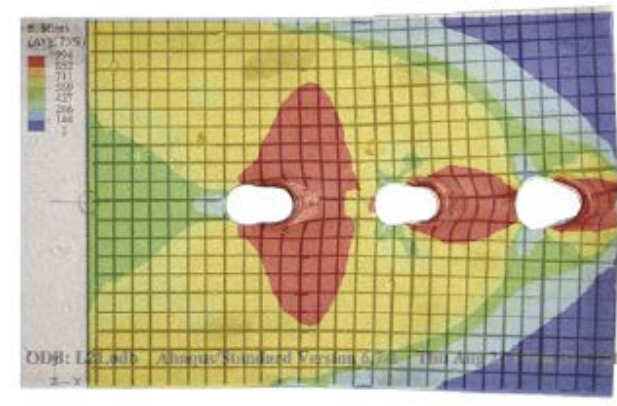

(b)

Figure 2-42 Von Mises stress plotted over the actual specimens (a) Net section failure with bolt B1 closest to the sheet end; (b) Bearing failure showing stress distribution becomes greater at bolts further from sheet end (Moze \& Beg, 2011)

The most obvious deficiency in most of the aforementioned FE models, is the restriction in the rotational degrees of freedom of the bolts, so as to prevent sliding of the plates along the bolt shanks when the bolts are modelled as rigid body elements. Thus, the FE results do not reflect tilting of the bolts, as is usually observed in testing. 
When compared to the body of work attributed to bolted connections, limited research reports exist regarding the numerical investigation of screwed connections. Yan (2012) attributed this to the difficulty in attaining convergence when the steel plate is less than $1 \mathrm{~mm}$ in thickness, combined with the complications of modelling the screw shank threading, and the uncertainty of the contact settings between the screws and plates.

\subsubsection{Fan, Rondal \& Cescotto (1997a; 1997b)}

Fan et al. (1997a; 1997b) modelled single lap screw connections in steel sheeting, under static shear. The LAGAMINE software used by Fan et al. (1997a; 1997b) is uncommon, though some of their original thoughts and contact laws are still thought to be of value when used presently. Their simulation aimed to model the behaviour of a screwed connection where failure is a result of yielding of the connected steel sheets, though not by failure of the screw itself.

The objective of the simulation was the computation of the stresses in the vicinity of the screw in the connected steel sheets, as well as to assess the global deformation and screw rotation as the connection deflected. Their model was calibrated against hundreds of screw connection tests using a $6.3 \mathrm{~mm}$ diameter screw, where the thickness of the steel sheet ranged between 0.63 and $8.0 \mathrm{~mm}$.

The entire connection was defined as 3 continuous bodies; firstly the thinner steel sheet, secondly the thicker steel sheet, and lastly, the screw shaft and head together with the aluminium alloy washer and the neoprene washer. Though realistically, the screw and washers do not form a continuous body, the researchers considered that linking the two would have very little influence on the behaviour of the connection, whilst appreciably reducing the computational time required. The primary function of the screw thread is the prevention of movement of the screw along its axial direction, but does not appreciably prevent rotation. In order to model this function of the thread, whilst still limiting the number of elements present, Fan et al. (1997a; 1997b) considered a simplified thread model for the screw, as illustrated in Figure 2-43.

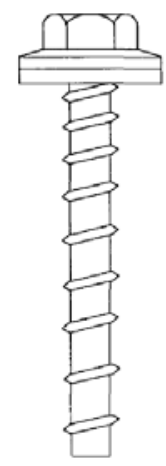

(a)

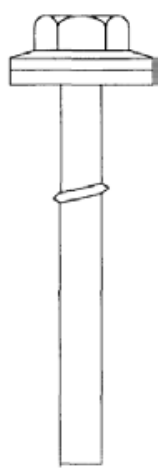

(b)

Figure 2-43 Screw thread simplification (a) real screw; (b) simplified screw (Fan et al., 1997a; 1997b) 
During loading, the researchers applied a uniform displacement with a maximum increment of $0.004 \mathrm{~mm}$ for each step. Due to the fact that Fan et al. (1997a; 1997b) were limited by their available computational resources, meshing of the model was achieved through a combination of trial and error, along with the utilisation of a very simplistic mesh for the analysis, in order to increase computational efficiency, as shown in Figure 2-44.

(a)
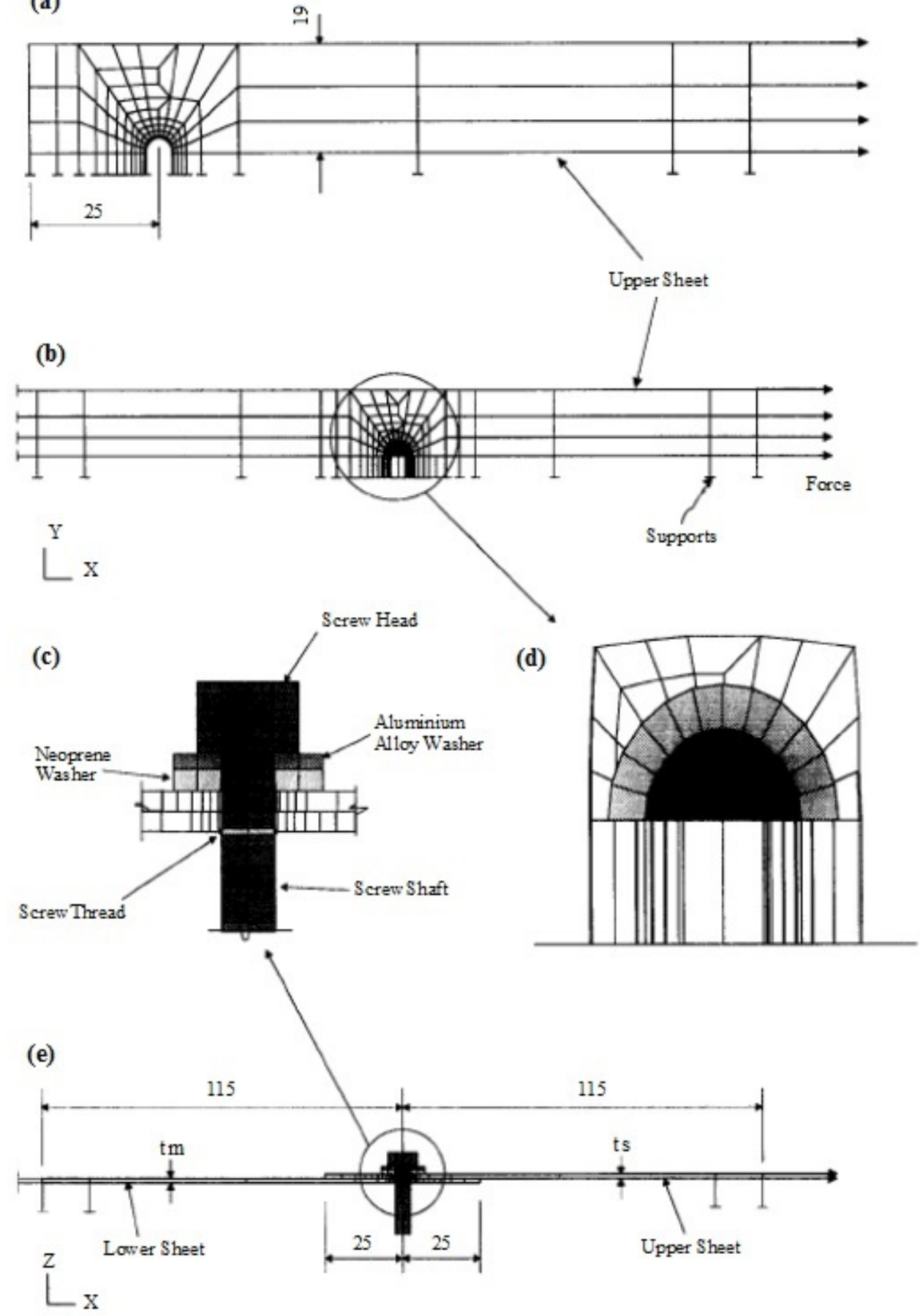

Figure 2-44 Finite element mesh, boundary conditions and applied force: (a) upper sheet; (b) structure top view; (c) detailed side view; (d) detailed top view; (e) structure side view (all dimensions in mm) (Fan et al., 1997a; 1997b) 
In order to control the plasticity of the materials, Fan et al. (1997a; 1997b) utilised the von Mises yield criterion. The material properties obtained from tensile coupon tests were converted to true stresses and strains. The mechanical properties of the screw shaft and head were based upon a linear elastic constitutive law; whilst the two steel sheets, aluminium alloy washer and neoprene washer were separately modelled, using four piecewise defined elastoplastic constitutive laws. During their trial simulations, it was found that the von Mises stress in the screw vicinity of the thinner sheet exceeded the ultimate tensile strength of the material, in connections where the ratio between the thicknesses of the sheets was large. This in turn meant that the material mainly under tension stress is already cracked, but continues to function under compressive stress. To treat these local cracks, the damage theory was utilised. Fan et al. (1997a; 1997b) noted that a damage law was unavailable for the elements they had used, and instead the true-stress-strain curve of the steel sheet was defined by two parts beyond the ultimate tensile strength; one for the material undergoing tension, and the other for the material under compression, as shown in Figure 2-45.

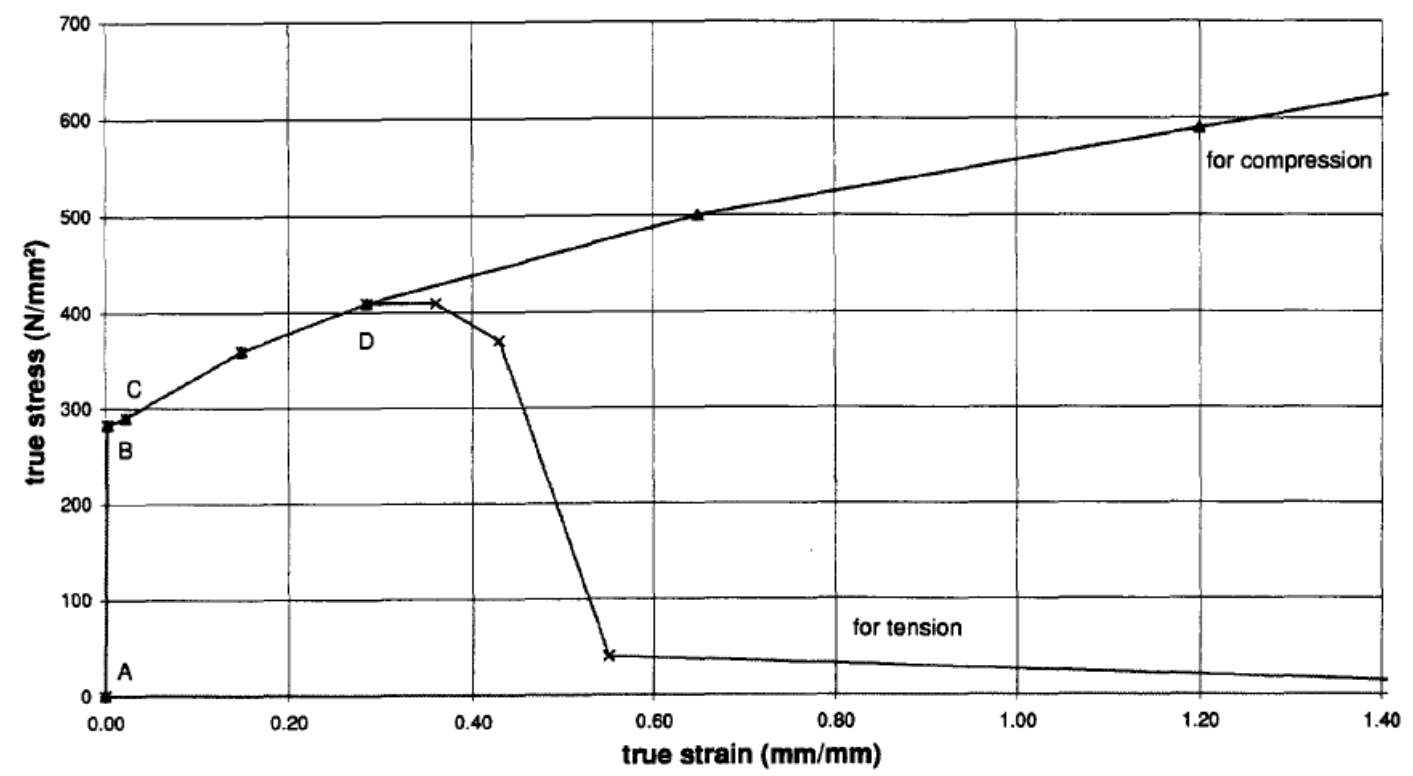

Figure 2-45 The mechanical properties of $0.63 \mathrm{~mm}$ steel sheet used in the numerical simulation. Curve ABCD was obtained from a tensile test (Fan et al., 1997a; 1997b)

A three-dimensional contact law was applied in order to simulate contact between the various elements, involving the use of three-dimensional contact elements. The penalty method (Appendix A) was used to enforce the contact condition. With the provision that the computation could converge; a penalty coefficient for the shear frictional stress and the contact pressure were chosen to be as high as possible, in order to avoid penetration between contacting materials. The penalty coefficients for the contact pressure and the frictional stress were both chosen as $3 \times 10^{4} \mathrm{~N} / \mathrm{mm}^{2}$ for contact between the thinner sheet and the screw shaft, and as $5 \times 10^{4} \mathrm{~N} / \mathrm{mm}^{2}$ for contact of the thinner sheet to the thicker sheet. 
Due to the fact that a simplified screw was used, Fan et al. (1997a; 1997b) made use of cohesion in order to effectively model a part of the screw thread function. The five contact laws used for the interfaces included:

- A friction and cohesion coefficient equal to 0.13 and 0 for the thin sheet to the thick sheet, respectively.

- A friction and cohesion coefficient equal to 0.25 and 5 for the screw shaft to the thin sheet.

- A friction and cohesion coefficient of 0.18 and 0 for the screw shaft to thick sheet, respectively. This could be reduced due to only the one thread being present, though in reality, thin contact should be the same as that between the thin sheet and the screw shaft.

- A friction and cohesion coefficient equal to 0.18 and 0 for the screw thread to the thick sheet, respectively.

\subsubsection{Explicit vs Implicit Dynamic Analysis Method}

Upon examination of the previous literature, it is interesting to note that in either bolted or screwed connections, the thickness of the steel plates is usually higher than $1.20 \mathrm{~mm}$. Thinner steel sheets, such as 0.8 and $0.42 \mathrm{~mm}$, are becoming increasingly popular in the fabrication of members and roof sheet structures. The thinnest steel sheets used for decking and roofing could even be as low as $0.35 \mathrm{~mm}$ in thickness. Numerical investigations into bolted and screwed connection assemblies where the thickness of the steel sheets is less than $1.20 \mathrm{~mm}$ are scarce.

When compared to the conventional static analysis method, the explicit dynamic analysis provides several advantages; one of which is the computational efficiency for large models with relatively short dynamic response times, as well as the analysis of extremely discontinuous events or processes. The explicit dynamic analysis can be used to perform quasi-static analysis with complicated contact conditions, whilst allowing for the definition of very general contact conditions (ABAQUS, 2015).

\section{Implicit Dynamic Analysis:}

The transient dynamic analysis method, occasionally referred to as a time-history analysis, can be used to determine the dynamic response of a structure, due to the effect of any type of loading, which varies with time. This method can determine the time-varying displacements, accelerations and velocities during each time step of the analysis (Mac Donald, 2011). The equation which is solved through the employment of the transient dynamic analysis is given in equation:

$$
[\boldsymbol{M}]\{\ddot{\boldsymbol{U}}\}+[\boldsymbol{C}]\{\dot{\boldsymbol{U}}\}+[\boldsymbol{K}]\{\boldsymbol{U}\}=\{\boldsymbol{f}(\boldsymbol{t})\}
$$


Where $[\mathrm{M}]$ denotes the global mass matrix; $\{\mathrm{U}\}$ the nodal displacement vector; $[\mathrm{C}]$ the global damping matrix; $[\mathrm{K}]$ the global stiffness matric and $\{\mathrm{F}(\mathrm{t})\}$ is the time-dependant load vector. During the solution, these equations also account for inertial and damping forces, and can be considered as a set of static equilibrium equations, for any time value, t. FEA solvers utilise time integration methods in order to solve these equations at discrete points, with the time increment between successive time points referred to as the "integration time step", or simply as the "time step". The majority of solvers utilise an implicit integration method, where the fundamental approach assumes that the solution for a discrete time value, $\mathrm{t}$, is known, and that the solution for a discrete time, $\mathrm{t}+\Delta \mathrm{t}$, is required where $\Delta \mathrm{t}$ is an appropriately chose time interval.

The FEA solver will increment a time step during the implicit time integration solution, and will iterate to determine if the loads or external forces applied to the structure equilibrate with the internal forces. Considering that each new time step is a function of the displacements and accelerations of the previous step, this necessitates that the equilibrium check be performed by iteration. Before the equilibrium calculations can commence, the calculation additionally requires the formation and inversion of the global model mass, damping and stiffness matrices.

\section{Explicit Dynamic Analysis:}

As previously stated, the implicit time integration method requires the formation and inversion of the global system equations at each additional step. This process is highly intensive computationally, especially when the model and the degrees of freedom become large, causing the CPU time increments to increase dramatically. This is not an issue when solving problems with few increments, but when a large number of time increments are required, it is highly likely that the computational resources available will quickly be depleted. (Mac Donald, 2011).

Thus, explicit time integration offers a surrogate method which can rapidly and efficiently solve such problems. During explicit integration, nodal accelerations are calculated directly using Newton's Second Law $(\mathrm{F}=\mathrm{ma})$, utilising a diagonal mass matrix. As the response of each node is independent, this eradicates the requirement to generate and invert large matrices, allowing the nodal displacements and velocities to be directly calculated from the time increments and accelerations that the solution has already progressed through. The term "explicit" is used because the calculation refers only to the values of acceleration, displacement, force and velocity at the initiation of each new time step. As the solution is deduced on an individual element basis, this enables large 3D models, comprising thousands of degrees of freedom, to be solved using much less computational power when compared to an implicit integration (Mac Donald, 2011).

The differences between the implicit and explicit methods can by fully explained through consideration of the equation below, which depicts the general form of the implicit method:

$$
U^{n+1}=f\left(\ddot{U}^{n+1}, \dot{U}^{n+1}, U^{n}, \ldots \ldots\right)
$$


Where the current nodal displacement, $U^{n+1}$, is a function of time derivative of $U^{n+1}$, which is the nodal accelerations and velocity, which are also unknown. Thus, the implicit method iterates a solution for the nodal displacements and its time derivatives, in order to solve the equations. This requires solving simultaneous equations and hence inverting the large system matrices (Mac Donald, 2011).

Conversely, the general form of the explicit method is depicted as:

$$
U^{n+1}=f\left(U^{n}, \dot{U}^{n}, \ddot{U}^{n}, U^{n-1}, \ldots \ldots\right)
$$

Where in this case, the current nodal displacement can be seen as a function of the nodal displacement, acceleration and velocity from the preceding time step. Considering that this formation is already known, this allows the use of a diagonal mass matrix, and additionally, the global equation is a system of linear algebraic equations, which can be solved for without having to resort to using simultaneous equations.

Large rotations and deformations can be modelled by an explicit dynamic solver using a consistent large deformation theory, providing an easy solution procedure. For each load increment in a static analysis, the continuity of the contact states are first checked. The stiffness matrix is adjusted if any of the contacts in the model changes state, and the increment is reperformed based on the changed state of contacts. Only once the continuity of all contacts are satisfied, can the force equilibrium be checked. At the beginning of each analysis, the algorithm imposes huge difficulty when many contact pairs are defined in the model, due to the challenge of finding a stable state for all the contact pairs at the same instant. In bolted or screwed connections, three contact pairs are generally defined per screw; contact between the screw shaft and the holes in each of the two connected plates, and contact between the head and plate surfaces. The analysis of a screwed structural assembly could likely involve $4-6$ screws, entailing a large number of contact surfaces, increasing the difficulty for the static analysis solver to attain convergence. Yu et al. (2008) recommended the use of an explicit dynamic solver to analyse the behaviour of bolted steel connections, as it avoids numerical convergence difficulties, as well as allowing the definition of very large general surface contacts with large deformations.

A kinetic contact algorithm is used in the explicit dynamic solver, allowing the model to move into a predicted configuration without consideration of the contact conditions. According to the amount of penetration, the equilibrium state is determined through compensation of the forces and displacements of the nodes on the contact surfaces. No convergence check is required, though several corrections may need to be performed in order to remove any residual penetration. Through limitation of the time increment for each step to a very small value, the stability of the analysis is maintained. The smallest transit time of a dilatational wave (see Appendix A) across any of the elements in the mesh provides an approximate estimate of the stability limit for the explicit time integration, and is thus proportional to the dimensions of the smallest element in the mesh (Yu et al., 2008). 
Due to the time required to finish an analysis being proportional to its time duration, it is computationally inefficient for quasi-static problems to utilise real time; thus explicit dynamic analysis provides the transient response of the problem being analysed. Though, if the response of the structure remains mostly static, it is often possible to scale the real time to a very small time period.

When a dynamic system is subject to a linearly rising load, its response, according to classical dynamic theory, can be approximately treated as static, provided that the duration of the loading stage is large when compared to the natural period of the system. This statement however, provides no direct advice regarding the exact time duration to use for complex structures; but rather provides a general implication that a time duration which has been shown to produce a quasi-static response for one problem, will typically be suitable for another, provided it possesses similar natural frequencies.

\subsubsection{Yu, Burgess, Davison \& Plank (2008)}

$\mathrm{Yu}$ et al. (2008) dealt with the complicated contact pairs in hot-rolled steel bolted connections, achieving strong correlations in the comparison of FEA and test results, through the adoption of the explicit dynamic analysis. They evaluated the effect of loading speed in a lap joint test connecting two plates, 9.5 and $12.7 \mathrm{~mm}$ thick, using one $19 \mathrm{~mm}$ bolt. The explicit dynamic simulated test was compared to a general static simulation, and a comparison of the different simulation results versus the test results is provided in Figure 2-46. If the loading is applied in 0.1 or 1 second increments, the explicit dynamic analysis results are smooth, and very close to those of the static analysis. When the loading is applied in 0.01 second increments, the response in the initial linear phase exhibits minor vibration, but in the elasto-plastic response phase becomes close to that of the static analysis.

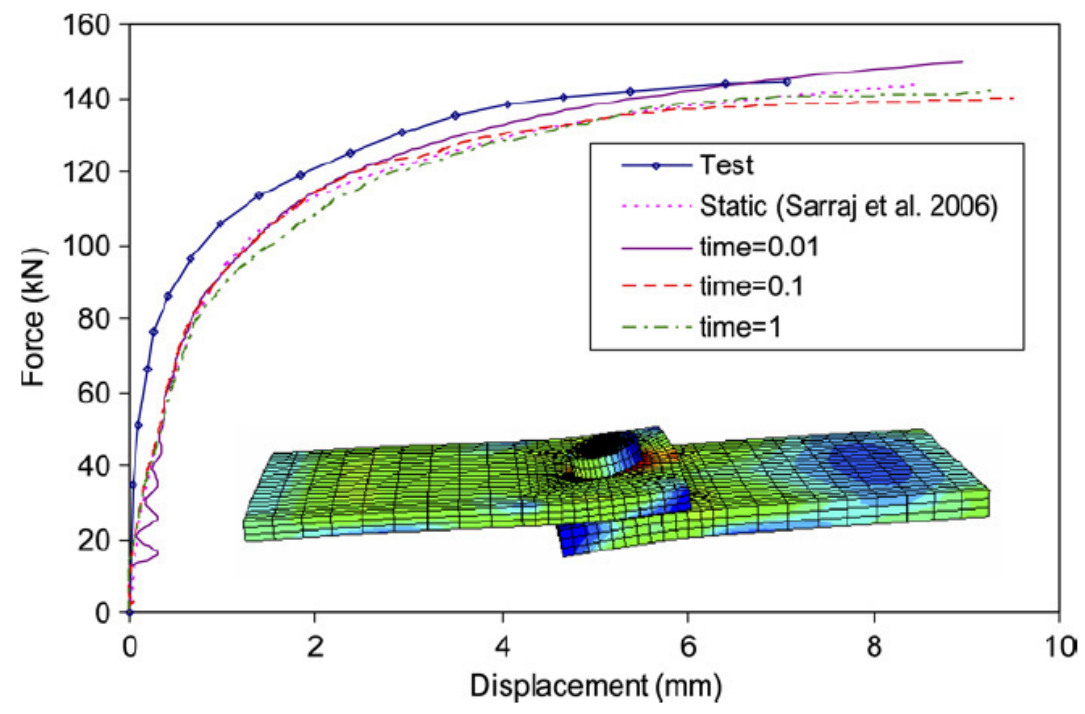

Figure 2-46 Comparison of test results from static analysis and explicit dynamic analysis for a lap joint test (Yu et al., 2008) 
Through controlling the loading speed, quasi-static problems can be simulated using the explicit dynamic analysis. However, two potential drawbacks are; when too rapid an increase in load is applied, the structural response becomes dynamic, and due to the inertial force assisting to balance the applied load, the post-failure resistance may be overestimated. Unfortunately, no quick fix exists for either problem, requiring a pragmatic approach to be adopted. When considering the dynamic structural response, a conclusion can be drawn from the previous two studies that, for normal steel connections, a loading time of between 0.1 and 1 seconds should be suitable for most applications. Yu et al. (2008) stated that the loading time should be increased proportionally in relation to the softness of the structure being analysed.

Yu et al. (2008) also studied the effects of variation of the mesh sizes representing the bolts and connected plates. Generally, the response to mesh size was not very sensitive, unless using extremely coarse meshes. Lastly, three sets of connection tests were simulated at ambient and elevated temperatures, using explicit dynamic analysis, and the results obtained correlated very strongly to the test results.

In order to check whether the structure has indeed failed, and the analysis is simply continuing as a dynamic motion, two methods can be employed. Firstly, the ratio of kinetic to internal energy should be examined, and as stated in the ABAQUS/Explicit manual (ABAQUS, 2015), if the ratio is less than $10 \%$, the procedure is quasi-static. Any responses exhibiting an energy ratio larger than this can automatically be assumed to be dynamic, and should be removed from the results. The second method is through checking the equilibrium of the reaction force and the applied load; if the reaction force is approximately equal to the applied load, the response is static. Once the structure approaches failure, the reaction force, as seen in the force-displacement graph, should decrease as the structure has become too weak to transmit the applied load to the boundary.

\subsubsection{Lu, Ma, Makelainen \& Outinen (2012)}

Lu et al. (2012) performed a FE analysis of screwed connections of thin steel sheets, using the explicit dynamic analysis method. In order to simulate the structural application of connecting roof sheeting to truss members, they investigated the performance of screwed connections between a thinner and a thicker steel plate. The connection in their simulation comprised of $5.5 \mathrm{~mm}$ diameter screws, with a $15 \mathrm{~mm}$ diameter built in washer, connecting $0.8 \mathrm{~mm}$ thick S350GD+Z steel sheeting to $5.0 \mathrm{~mm}$ thick S355 steel plates, using the FE software ABAQUS/Explicit as an analysis tool.

In order to improve the computational efficiency of their analysis, three threads were modelled along the screw shank. The thick plate, the thin sheet, the screw, the screw thread and the washer were all modelled using three dimensional eight-node solid elements. In the vicinity of the screw connectors, the mesh densities in the two sheets were refined, due to deformation primarily originating from this area and the local buckling of the thinner sheet. Their FE model is shown in Figure 2-47. The right end of the thick plate was fixed, with loading applied to the edge of the thin plate, up to a maximum of $15 \mathrm{~mm}$, through means of a displacement controlled method. General contacts were provided for all contact surfaces. 


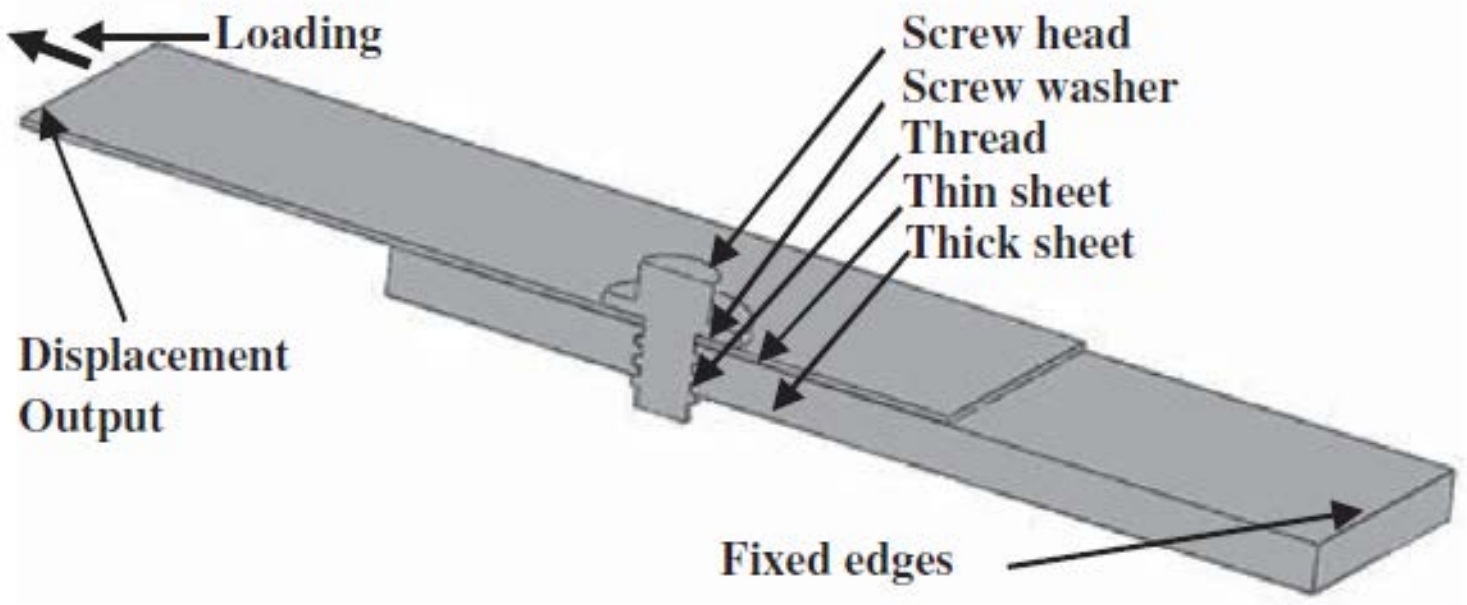

Figure 2-47 FE model of single lap screw sheeting connection (Lu et al., 2012)

No damage was defined in the material model for the thick S355 grade steel plate, as test results had shown that damages were mainly concentrated on the thin sheet in the connection area. The onset of failure for the steel sheet was defined at a true strain of 0.45 , whilst damage reached $90 \%$ at a plastic strain of 0.5 , with complete material failure occurring at a plastic strain of 1 . It should be noted that the damage model provided for the thin sheet steel in the connection was determined through experimental testing. The true stress and true strain curves, with damages defined for the thin S350, are shown in Figure 2-48.

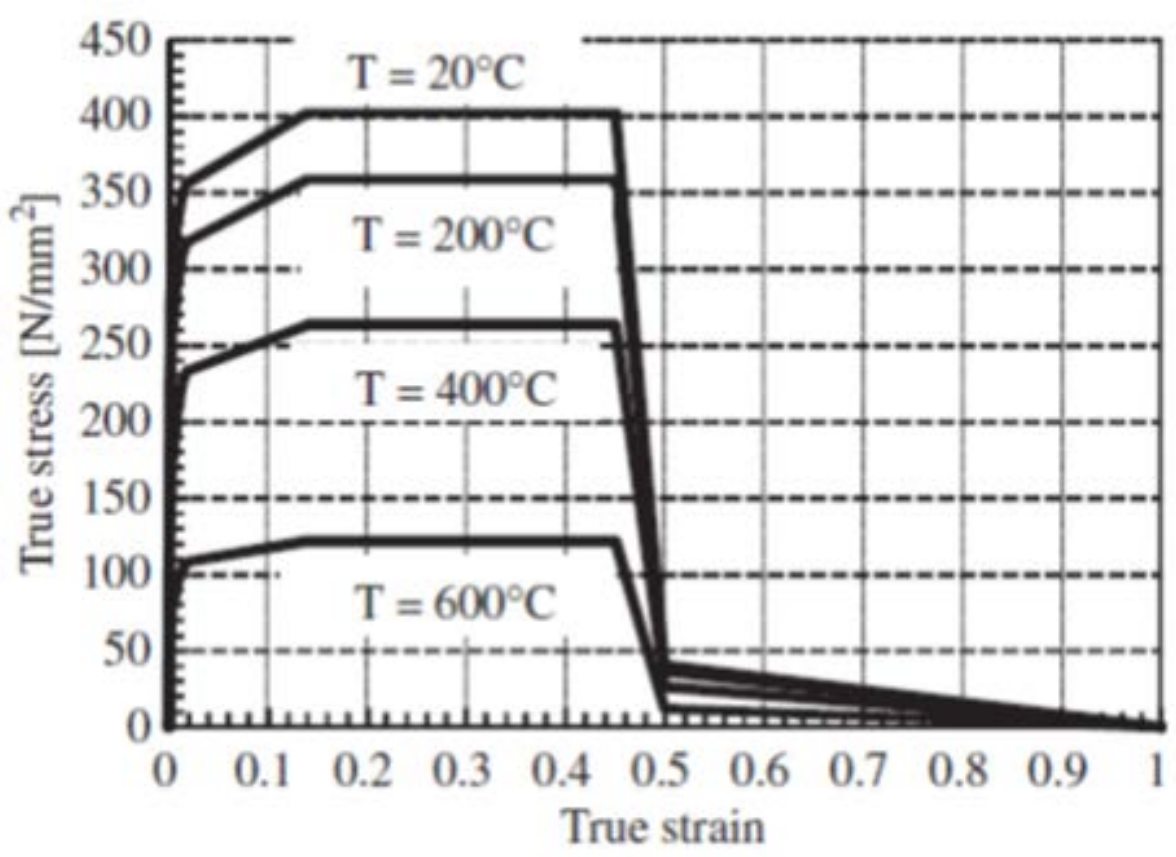

Figure 2-48 Material model at room and elevated temperature for S350 steel (Lu et al., 2012) 
At ambient temperatures, the failure mode observed for the screwed connections was found to be a bearing type failure, which can be seen in Figure 2-49, along with the load-displacement curves. As illustrated, the FE analysis correlated satisfactorily with those obtained from testing, in terms of both the shape of the curve, as well as the maximum value of the forces. In this analysis, it should be noted that pre-stress in the screw was included. The FE analysis revealed that, under shear loading, the failure mode of the connections was through bearing failure of the thin sheet. It was found that the load bearing capacity of the connection depended upon the friction between the washer and the thinner sheet, the post buckling resistance of the thinner sheet and the direct bearing of the screw shank against the thinner sheet.
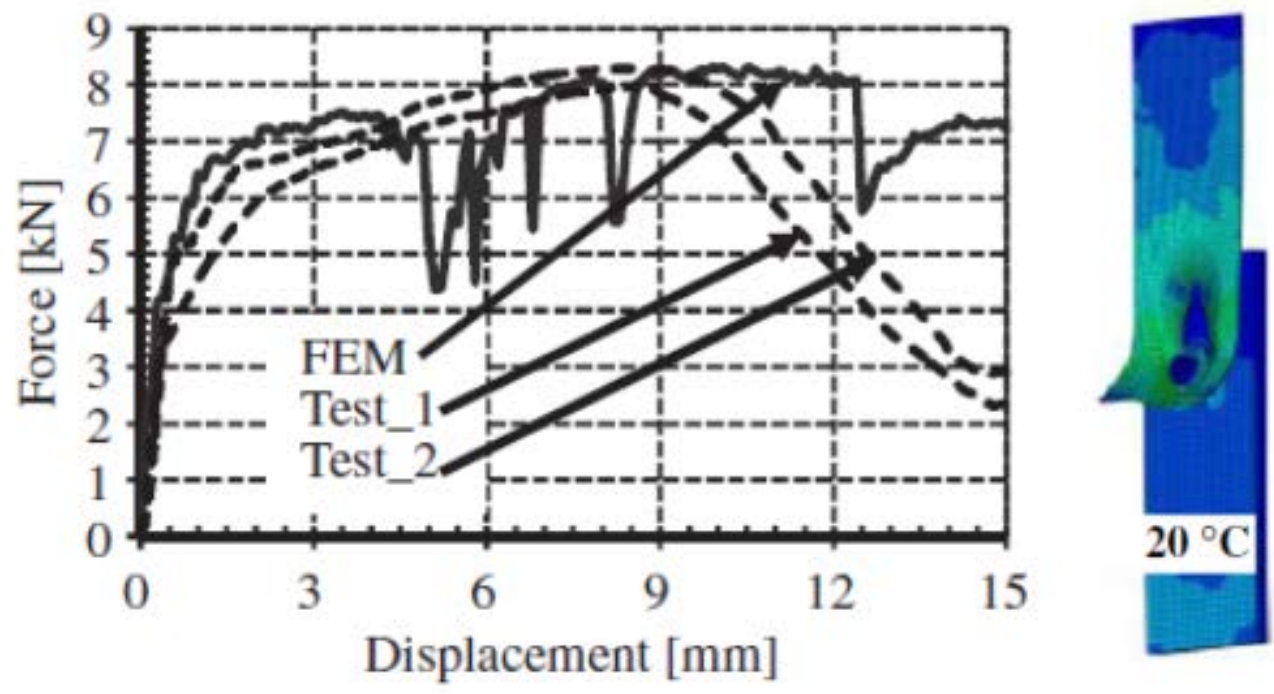

Figure 2-49 Load displacement curves and failure mode observed for screwed connections (Lu et al., 2012)

\subsubsection{Yan (2012)}

Yan (2012) used the ABAQUS/Explicit Dynamic Analysis to perform a numerical study on the behaviour of single shear screwed connections of thin sheet steels, at both ambient and elevated temperatures. The thickness of the thin sheet steels utilised in the FEA ranged between 0.35 and $3.2 \mathrm{~mm}$, using G450, G500 and G550 grade steels. The material nonlinearities of the thin sheet steels, as well as the geometry of the connections and the interactions between the steel sheets and fasteners, were all carefully incorporated into the FE models. Numerical analysis was used to obtain the ultimate strengths, as well as the various failure modes of the single shear screwed connections. The verified finite element model was used in an extensive parametric study, investigating the tilting and bearing strengths of single shear screwed connections of thin sheet steels at elevated temperatures. The parameters which were considered included; the thickness of the steel plate, the distance from the centre of the screw hole to the end of the plate, as well as the screw size, number, arrangement and spacing. 
Three-dimensional eight-node linear brick elements were used to model the steel plates and screws, with the size of the elements in the FE mesh in the overlapped part of the connection ranging from a length and width of $1.5 \times 1.5 \mathrm{~mm}$ to $2.5 \times 2.5 \mathrm{~mm}$, depending on the length of the overlapped section. Around the bolt holes, a finer mesh size comprising 32 elements was used, with the mesh becoming gradually coarser along the longitudinal direction towards the end of the specimen.

The experimental test set-up is displayed in Figure 2-50, and as the connection specimens were gripped at the ends, the boundary condition of the end pins were simulated through restriction of the freedom of the two reference points, RP-1 and RP-2, as illustrated in Figure 2-51. Except for the in-plane rotation, all degrees of freedom at reference point RP-1 were restrained. At reference point RP-2, the in-plane rotational degree of freedom and the translational degree of freedom in the longitudinal axial direction were released, with all other degrees of freedom restrained. The loading method employed in the FEA was identical to that used in the tests, with the tensile axial load applied to the FE model through specification of the longitudinal axial displacement at reference point RP-2. The movement of RP-2 in the longitudinal displacement was equivalent to the movement of the hydraulic actuator, as recorded in the experimental tests. In order to prevent the extent of out-of-plane curling at the end of the steel plate in the overlapped part, the nodes in the out-of-plane displacement at the edges of the plates in a certain width were restrained, in order to simulate the effect of clips, as shown in Figure 2-51. Screw hole deformation was monitored through the insertion of two reference points, as indicated at points $\mathrm{A}$ and $\mathrm{B}$.

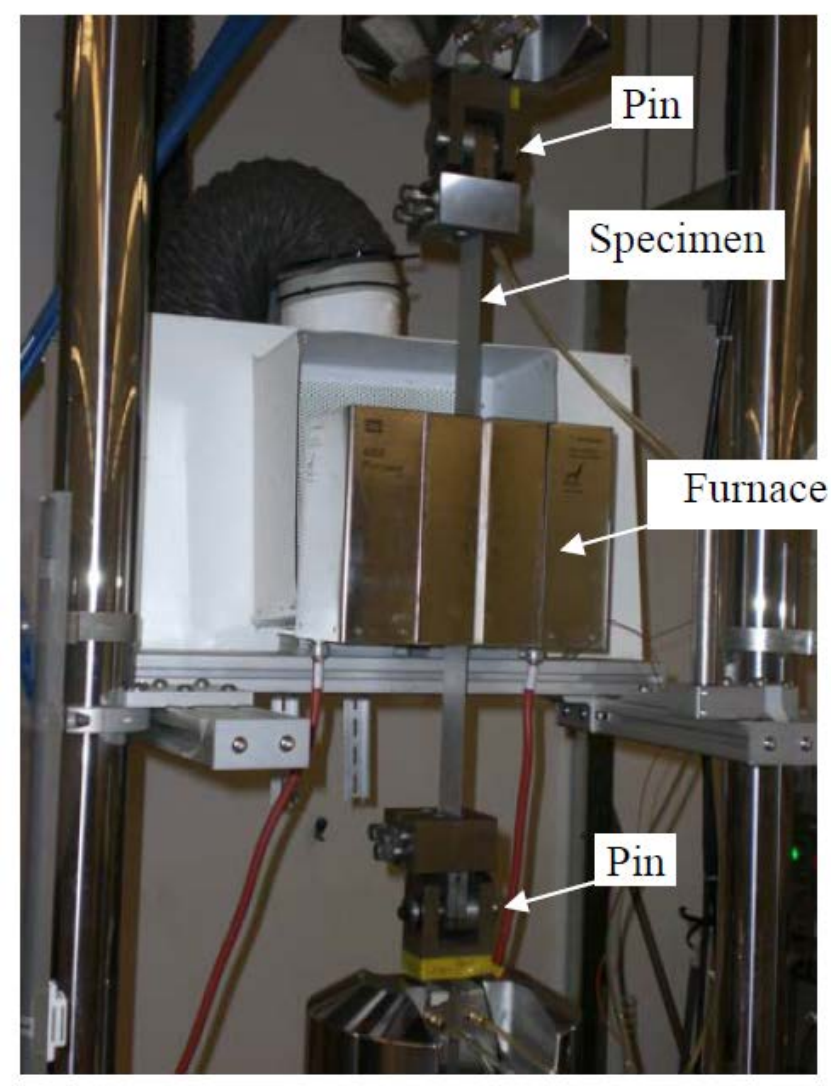

Figure 2-50 Test set-up of single shear screwed connections of thin sheet steels at elevated temperatures (Yan, 2012; Yan \& Young, 2012) 


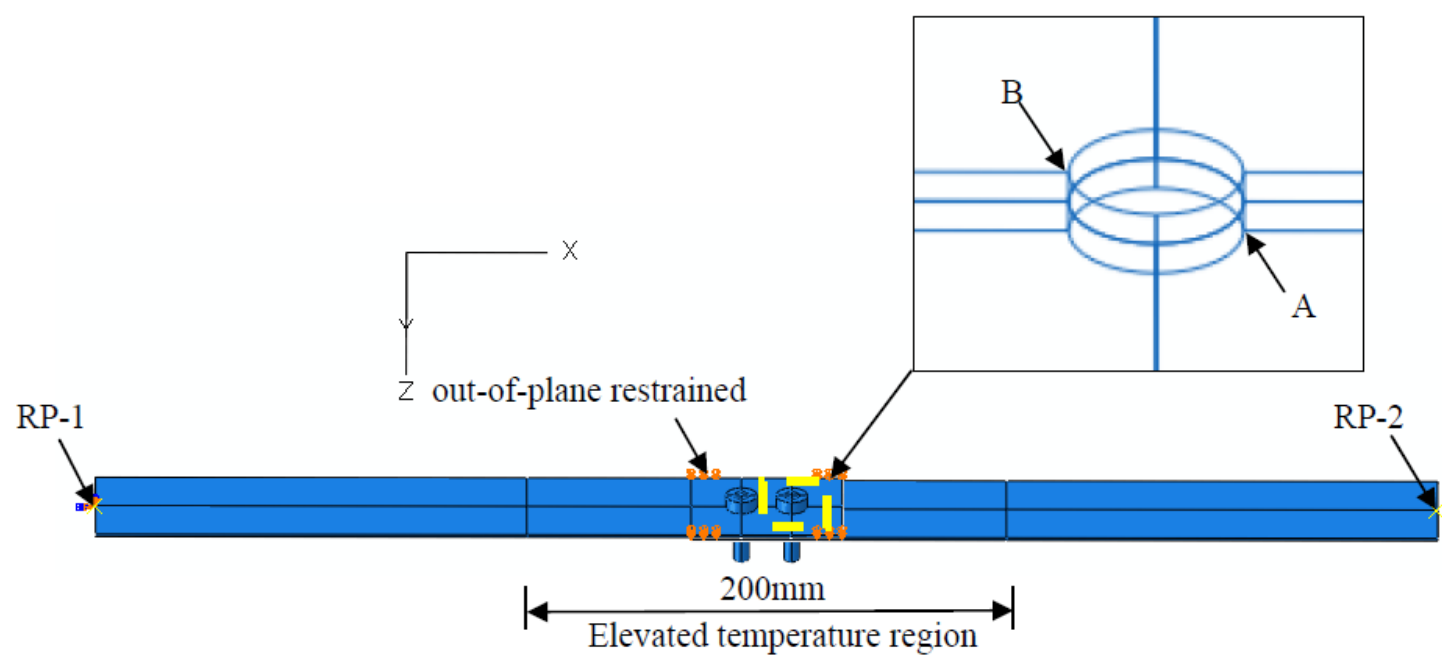

Figure 2-51 Boundary conditions and reference nodes of FE model (Yan, 2012)

Contact pairs were used to define the interfaces between the different contact zones, with the screws assumed to be the master elements, and the steel plates the slave elements of the interface elements (see Appendix A). The two contact surfaces were prevented from penetrating each other, with all contacts defined as surface-to-surface contacts, with a finite sliding option, and "hard contact" used as the normal contact behaviour. The friction coefficient of 0.13 was defined in the tangential direction between the steel sheet plates. The pre-stress to the screws was not considered, and the threads along the screw shank were not modelled, and instead a relatively high friction coefficient of 0.40 was defined between the screw shank and the screw holes. The reduced friction coefficient of 0.13 was defined between the screw shank and screw holes drilled in the G550, $0.42 \mathrm{~mm}$ thin steel plates in cases where the spacing of the threads on the shank exceeded the thickness of the thinnest steel sheet. For the models tested at ambient temperatures, a loading rate of $1.0 \mathrm{~mm} / \mathrm{sec}$ was used. The material properties obtained from the tensile coupon tests were converted to true stress and true plastic strains. The material properties measured from coupon tests are given in Table 2-9.

Table 2-9 Coupon test results at normal room (ambient) temperature (Yan, 2012; Yan \& Young, 2011)

\begin{tabular}{cccccc}
\hline Series & $\begin{array}{c}E_{\text {nonmal }} \\
(\mathrm{GPa})\end{array}$ & $\begin{array}{c}f 0.2, \text { nonwal } \\
(\mathrm{MPa})\end{array}$ & $\begin{array}{c}f_{\text {Ll nonwal }} \\
(\mathrm{MPa})\end{array}$ & $\begin{array}{c}\varepsilon_{u, \text { nonmal }} \\
(\%)\end{array}$ & $\begin{array}{c}\varepsilon_{f, \text { normal }} \\
(\%)\end{array}$ \\
\hline $\mathrm{G} 5500.42 \mathrm{~mm}$ & 210 & 718.3 & 718.7 & 0.6 & 2.8 \\
\hline $\mathrm{G} 5001.20 \mathrm{~mm}$ & 215 & 613.7 & 627.3 & 4.5 & 9.0 \\
\hline $\mathrm{G} 4501.90 \mathrm{~mm}$ & 213 & 504.5 & 543.0 & 7.0 & 13.1 \\
\hline
\end{tabular}


In the verification of the FEM, a total of 38 single shear screwed connections of thin sheet steels were analysed. The initiation and propagation of brittle cracks which were observed in the tests were noted to be barely emulated in the FEM. The curve of the ratio of kinetic energy to internal energy (K/I) versus time, as well as the curve of the reaction force versus displacement obtained from the FEA results are shown in Figure 2-52. As depicted on the graphs, the $\mathrm{K} / \mathrm{I}$ value is less than $2 \%$, and the comparison of the reaction force to the applied load is in the range of 0.95 to 1.05 . The parametric study concluded that determination of the tilting and bearing strengths of single shear screwed connections, is conservative when utilising a $3 \mathrm{~d}$ spacing and end distance, where $\mathrm{d}$ is the screw diameter.

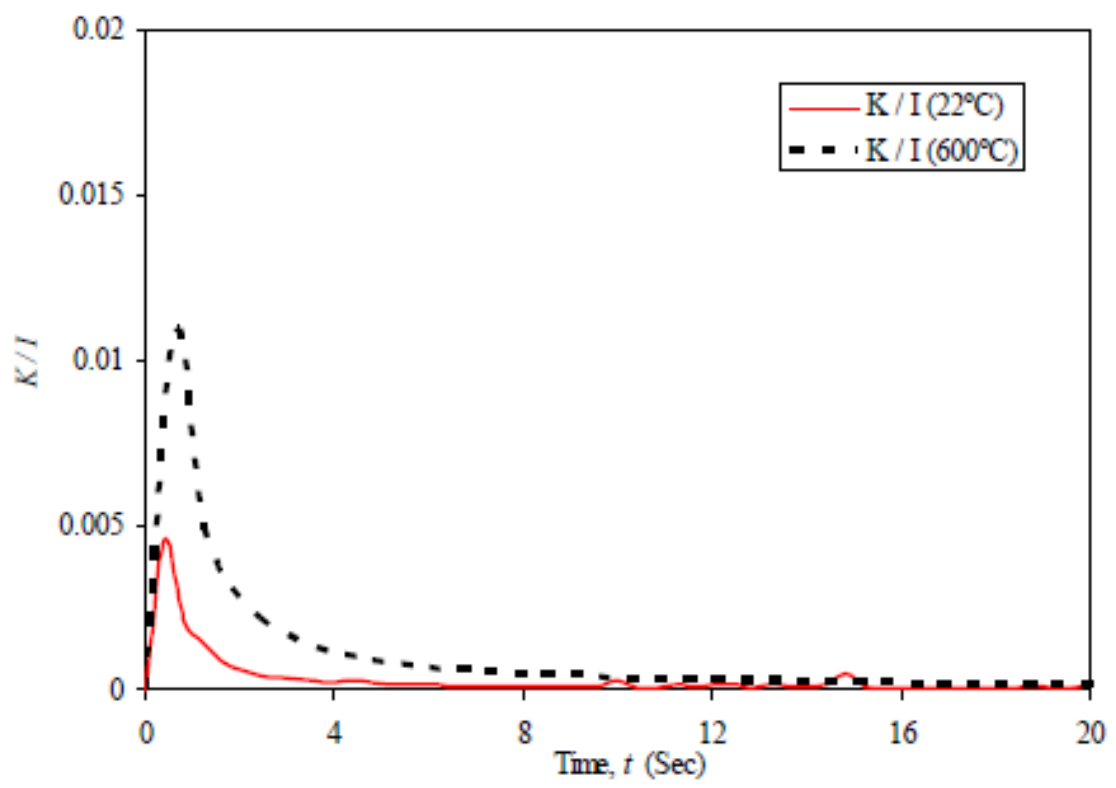

(a) $K / I$

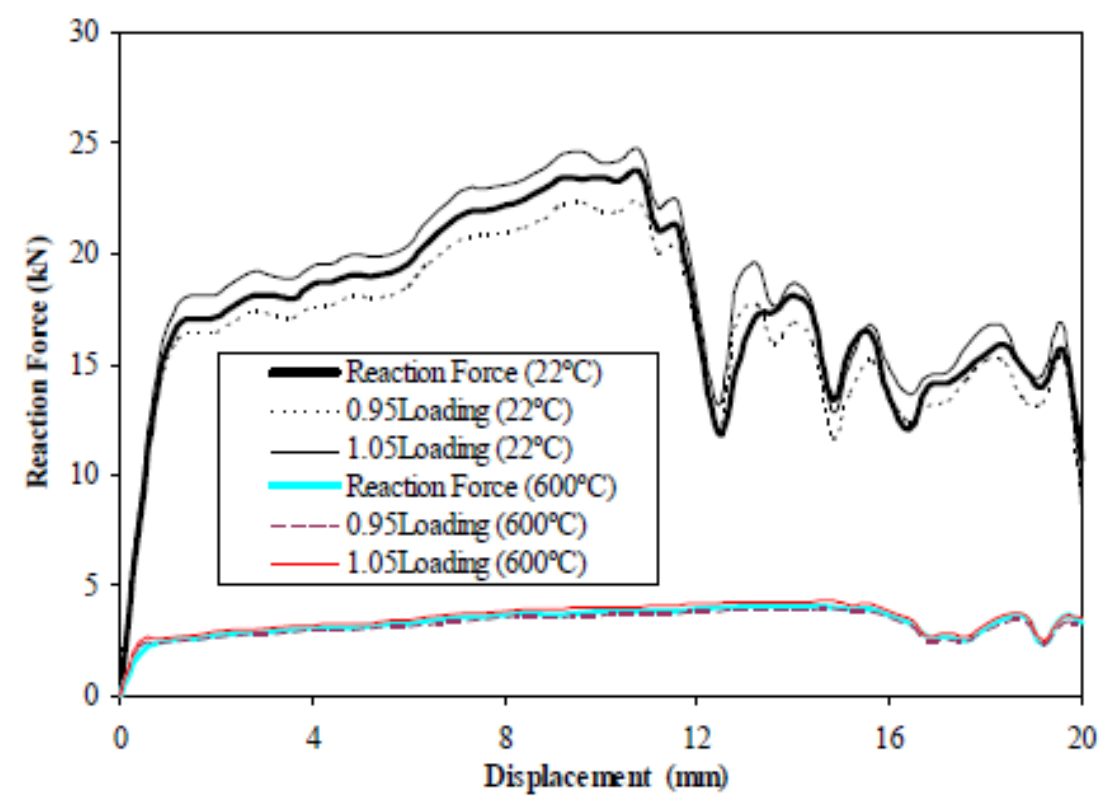

(b) Reaction force

Figure 2-52 FEA curves for $120-190$ screwed connections at $22^{\circ} \mathrm{C}$ and $600{ }^{\circ} \mathrm{C}$ (Yan, 2012) 


\subsection{SUMMARY}

The objective of this chapter was to highlight the previous research that has already been conducted by many researchers over the course of the last five decades. Initially, a brief overview was provided regarding the origins of cold-formed steel, and the basic theory behind how screwed connections operate. This was then followed by a more in-depth study regarding the provisions of cold-formed steel connections, and how they fail in terms of shear, tilting and bearing, as well as how the net section fails in tension.

Thereafter, the behaviour of screwed connections was examined, with regards to the effect of varying the thickness and ductility of the steel sheets. Furthermore, the connections were examined in order to determine what effect an increase in the number and patterning of screws involved in the connection had on both the strength of the connection, as well as the final mode of failure. The final section of this chapter examined the finite element analysis of lap shear connections, and presented the models that the previous researchers used, as well as the results that they obtained.

The initial finite element models presented illustrated the non-linear static modelling of lap shear bolted connections, and the conditions required to obtain convergence are summarised below:

- Due to the large disparities in the plate thicknesses, Chung \& Ip (2000; 2001) modelled the bottom plate as a single, solid rigid element. Furthermore, the displacement of the top sheet, where the load was applied, was limited to $3 \mathrm{~mm}$.

- Kim \& Kuwamura (2007), and subsequently Kim, Kuwamura \& Cho (2008), eliminated the bottom sheet entirely, and did not incorporate the bolt head geometry into their models by only making use of cylindrical shaft to model the bolts.

- Salih, Gardener \& Nethercot (2010) modelled thick plates (8 and $10 \mathrm{~mm}$ thick) and further stated that it was extremely difficult to attain convergence in such models. This was due to the program they utilised often terminating due to numerical instability, before an entire solution could be obtained.

- Moze \& Beg (2011) further simulated double shear bolted connections, with minor simplifications to the FE models, which corresponded well to their experimentally obtained results for many different types of failures. Numerical simulation in conjunction with testing indicated that high strength steel connections possessed sufficient ductility to redistribute the loading, even when the position of the bolts was unfavourable. However, due to the restriction of the rotational degrees of freedom of the bolts, the FE results do not reflect the tilting which is usually observed in testing.

- In all these models it is overtly evident that screw rotation was omitted from the modelling procedure. This is an important aspect of the modelling of screw connections, as one of the failure modes is a combined bearing/tilting failure.

- Furthermore, it should be noted that only sheets with a thickness greater than $1.5 \mathrm{~mm}$ was considered in these models. 
Fan, Rondal \& Cescotto (1997a; 1997b) developed the archetype finite element model for thin sheet steel screw connections whilst making use of a non-linear static analysis. Their model utilised a rudimental mesh in addition to other simplifications to the modelling procedure, such as the use of the simplified screw, and compensated for this through the inclusion of cohesion and friction; this allowed the authors to allow for screw rotations in the connections.

In order to include large rotations and deformations into the modelling procedure, the explicit dynamic solver provided a possible solution, through the use of large deformation theory. $\mathrm{Yu}$ et al. (2008) also recommended the use of an explicit dynamic solver in order to analyse the behaviour of bolted/screw steel connections, as it avoids numerical convergence difficulties, as well as allowing the definition of very large general surface contacts with large deformations.

Additional single lap shear connection models utilising this solver were presented finally, with their various modelling considerations, and are summarised as follows:

- Yu et al. (2008) dealt with the complicated contact pairs in hot-rolled steel bolted connections, achieving strong correlations in the comparison of FEA and test results, through the adoption of the explicit dynamic analysis. They further compared the use of the non-linear static vs the explicit dynamic procedure and found that they correlated closely if the load was applied in 0.1 to 1 second increments. They also identified two potential drawbacks to this method; either when the load was increased too rapidly, or that the inertial forces assisted to balance the applied load; and neither was amenable to a simple solution.

- Lu et al. (2012) performed a FEA of screwed connections between a thinner and a thicker steel plate, using the explicit dynamic analysis method, to simulate the structural application of connecting roof sheeting to truss members. The FEA correlated satisfactorily with those obtained from testing, in terms of both the shape of the curve and the maximum value of the forces. The FEA revealed the failure mode of the connections was through bearing failure of the thin sheet, and found that the load bearing capacity of the connection was dependent on; the friction from the washer, the direct bearing of the screw shank against, and the post buckling resistance of the thin sheet.

- Yan (2012) used the ABAQUS/Explicit Dynamic Analysis to perform a numerical study on the behaviour of single shear screwed connections of thin sheet steels, ranging in thickness from 0.35 to $3.2 \mathrm{~mm}$, at both ambient and elevated temperatures. In the verification of the FEM, a total of 38 single shear screwed connections of thin sheet steels were analysed, with the loading method employed in the FEA identical to that used in the tests, with the tensile axial load applied to the FE model through specification of the longitudinal axial displacement. The parametric study concluded that determination of the tilting and bearing strengths of single shear screwed connections, is conservative when utilising a $3 \mathrm{~d}$ spacing and end distance, where $\mathrm{d}$ is the screw diameter. 


\section{$3 \quad$ FINITE ELEMENT MODEL}

This chapter will detail the finite element model which was developed and subsequently used in the parametric study, as presented in chapter 4 . The finite element model will be defined in terms of the numerous modelling considerations which included: the types of elements used and the finite element mesh, the constraints, the material modelling and the interactions between the elements. The penultimate section of this chapter entails the verification of the FEM, and will show how it was calibrated against the work of Yan (2012).

A numerical study was performed on single shear screw connections of thin sheet steels using finite element analysis. The explicit dynamic analysis was utilised in the numerical investigation in this chapter. The material nonlinearities of the thin steel sheets and the geometry of the single shear screw connections were carefully incorporated into the finite element model. The primary objective of this chapter is the development and verification of the finite element model with the focus on the non-linear finite element modelling procedure, utilising the explicit dynamic analysis technique, and how accuracy is affected by various modelling parameters.

\section{$3.1 \quad$ INTRODUCTION}

Compared to physical experimental methods, finite element analysis affords an inexpensive, time efficient alternative. The only major drawback is the requirement for accurate test data in order to calibrate a finite element model. If the accuracy and validity of the data is assured, a finite element model allows the investigation of the structural behaviour against a wide range of parameters

The verification process for a finite element model should encompass some or all of the following steps:

- Quality assurance - Model convergence

- Comparison of results with expected results

- Comparison of results with hand calculations

- Using a model hierarchy

- Comparison to experimental results

- Comparison to the work of others

It has been documented that the computer modelling of screwed or bolted connections is a rather complicated task. Attention must be paid to: the significant geometrical and material non-linearities; contact between the components; and the possibility of localised fracture in the vicinity of the screw, at loading levels approaching the ultimate resistance of the connection.

The explicit solution method is a true dynamic procedure, and was originally developed in order to model high-speed impact events, where inertia plays a dominant role in the solution. When solving for a state of dynamic equilibrium, out-of-balance forces propagate as stress waves between neighbouring elements. As the minimum stable time increments are usually fairly small, most problems require a large number of increments. 
The explicit solution method has also proven valuable in the solving static problems. The major advantage of the explicit procedure, when compared to the implicit solution method, is the relative ease with which it resolves complicated contact problems. However, application of the explicit dynamic procedure to quasi-static problems requires some special considerations. By definition, a static solution is a long-time solution, and the analysis of the simulation in its natural time scale is often computationally impractical, often requiring an excessive number of small time increments. In order to obtain an economical solution, the event must be accelerated in some form or another. It should be noted that as the event is accelerated, the state of static equilibrium evolves into a state of dynamic equilibrium, as the inertial forces become more dominant. Therefore, the objective is to model the process in the shortest possible time, so that the inertial forces remain insignificant.

The finite element program ABAQUS V6.13 was used in the analysis for the simulation of single lap shear screw connections of thin sheet steels. Four main components have been carefully considered in the FEM; the steel plates, the screws, the interfaces between these two components, and the loading method.

\subsection{MODELLING CONSIDERATIONS}

In order to develop an appropriate finite element model, it is necessary to identify all possible physical actions involved within the structural system under consideration. The following actions are dominant in lap screwed or lap bolted connections.

\subsubsection{Nature of the Problem}

The problem at hand is a 3D static one. From the experimental investigations presented in Chapter 2, the finite element model is expected to include large material, geometrical and contact nonlinearities. Local plastification, as noted by Rogers and Hancock (1997; 1999), in the form of local cracks of the connected steel sheet in the screw vicinity would occur at the high loading stage in order to reach the ultimate resistance of the connection.

\subsubsection{Geometry and simplification of model}

The primary function of a structural connection is the transfer of a load from one structural component to the next. Depending upon the type of connection and the applied load, the connecting elements, such as the screws and bolts, may be subject to; compression, tension, shear, torsion and bending. In lap connections, the load is transferred from one plate to another primarily by means of shear transfer through the screws. The form of the structural assembly used in the experimental analysis is chosen in the numerical simulation, and is simplified in order to increase computational efficiency.

Most finite element studies are not interested in computing the stresses in the screws, since screw failure is not considered; but in order to obtain the screw displacement during loading so that screw rotation could be modelled, the screws also have to be discretised to be involved in the model. The screws were regarded as a linear elastic material, with approximately the same Young's modulus as the connected steel sheets. 


\subsection{TYPE OF ELEMENT AND FINITE ELEMENT MESH}

The 3D shell element has been widely used in sheeting modelling, due to the element being thin and subjected to simultaneous bending and membrane stresses. From the experimental studies presented in the Literature Review, it was observed that the inelastic strain in the connected sheets in the screw vicinity could be high. In the area below the screw head, the sheet is subjected to transverse compression, which is incompatible with shell hypothesis where the transverse stress is assumed to be zero (Fan et al., 1997a; 1997b). It is for this reason that shell elements are considered to be inappropriate for the analysis of screw connections, necessitating the use of 3D solid elements. Solid elements can be utilised in both linear analysis and complex nonlinear analysis involving contact, plasticity and large deformations.

\subsubsection{Elements}

The steel plates and screws were modelled using C3D8R solid elements. The C3D8R element is defined as a three dimensional, hexahedral 8-node linear brick element, with reduced integration (1 integration point) and hourglass control. First order elements are recommended when large strains, or very high strain gradients, are to be expected (ABAQUS, 2015). The C3D8R element has three translational degrees of freedom per node; $u_{1}, u_{2}, u_{3}$, and as for the element output, the stress components are as follows:

- $\quad \mathrm{S} 11$ ( $\sigma_{11}$; local 11 direct stress),

- $\quad$ S22 ( $\sigma_{22}$; local 22 direct stress),

- $\mathrm{S} 33$ ( $\sigma_{33}$; local 33 direct stress),

- $\quad$ S12 ( $\tau_{12}$; local 12 shear stress),

- $\quad \mathrm{S} 23\left(\tau_{23}\right.$; local 23 shear stress), and

- $\operatorname{S13}\left(\tau_{13}\right.$; local 13 shear stress).

Reduced integration utilises a lower-order integration to form the element stiffness, however, the mass matrix and distributed loadings are still fully integrated. Reduced integration reduces the running time, especially in three dimensions. Additionally, the linear reduced-integration element, C3D8R, is highly tolerant of distortion, allowing a fine mesh to be utilised in areas where high levels of distortion are expected (ABAQUS, 2015). It should be noted that hourglassing can be problematic with this first-order reduced integration element, since the elements only have one integration point. This means that the elements could distort in a manner enabling all the strains calculated at the integration points to be zero, leading to uncontrolled distortion of the mesh. This mode of deformation is termed zero-energy mode, as no strain energy is generated through element distortion, and can propagate through the mesh, producing inaccurate results (Kim \& Kuwamura, 2007). In order to counteract this, first-order reduced-integration elements in ABAQUS include hourglass control, where an artificial stiffness is added to the element, preventing excessive deformation. 


\subsubsection{Mesh}

The experimental studies showed that the deformation of a tested specimen originates primarily from the local area of the connected steel sheets behind the screw, and that this area exhibits buckling at high levels of loading. Fan et al. (1997a; 1997b) observed that the sheet was slightly sliding and bending up against the screw in the case of large screw rotation at ultimate loading levels. This necessitates intensive refinement of the mesh in this area not only due to the large strain gradient, but also the possibility of sheet buckling in order to prevent the individual elements from warping too much, to fit sheet curvature and not be subject to dominant bending. Elements further away from the screw can thus possess a large aspect ratio or a corner angle much larger than $90^{\circ}$. These shape distortions do not affect the results, due to the strain gradients in those areas being very small, and though these elements produce slight errors, they die out locally before propagating.

The computational cost of using the explicit method is proportional to the number or elements, and approximately inversely proportional to the dimensions of the smallest element. Hence, in areas of high mesh density, such as in the vicinity of the screw, the dimensions of the smallest element should be controlled in order to improve the computational efficiency.

The average size of the finite element mesh was $1.5 \mathrm{~mm}$ in the overlapped part of the connections, with a finer mesh comprising 40 elements in the vicinity of the screw hole (Figure 3-1). The mesh became gradually coarser in the longitudinal direction towards the end of the specimen. Two elements were utilised in the through thickness of the steel sheets. The mesh which was utilised in this study was carefully designed according to the specifications used by Yan (2012), in order to replicate his model as closely as possible. It should also be noted that the elements utilised in this study were in most cases smaller than those used by Yan (2012). As Yan's (2012) mesh utilised two elements when specifying the thickness of his model, and his model was indeed calibrated against over 500 experimental samples, in addition to the 490 parametric studies. It was deemed that further calibration of the mesh would not be required, since the validity of the model was already confirmed.

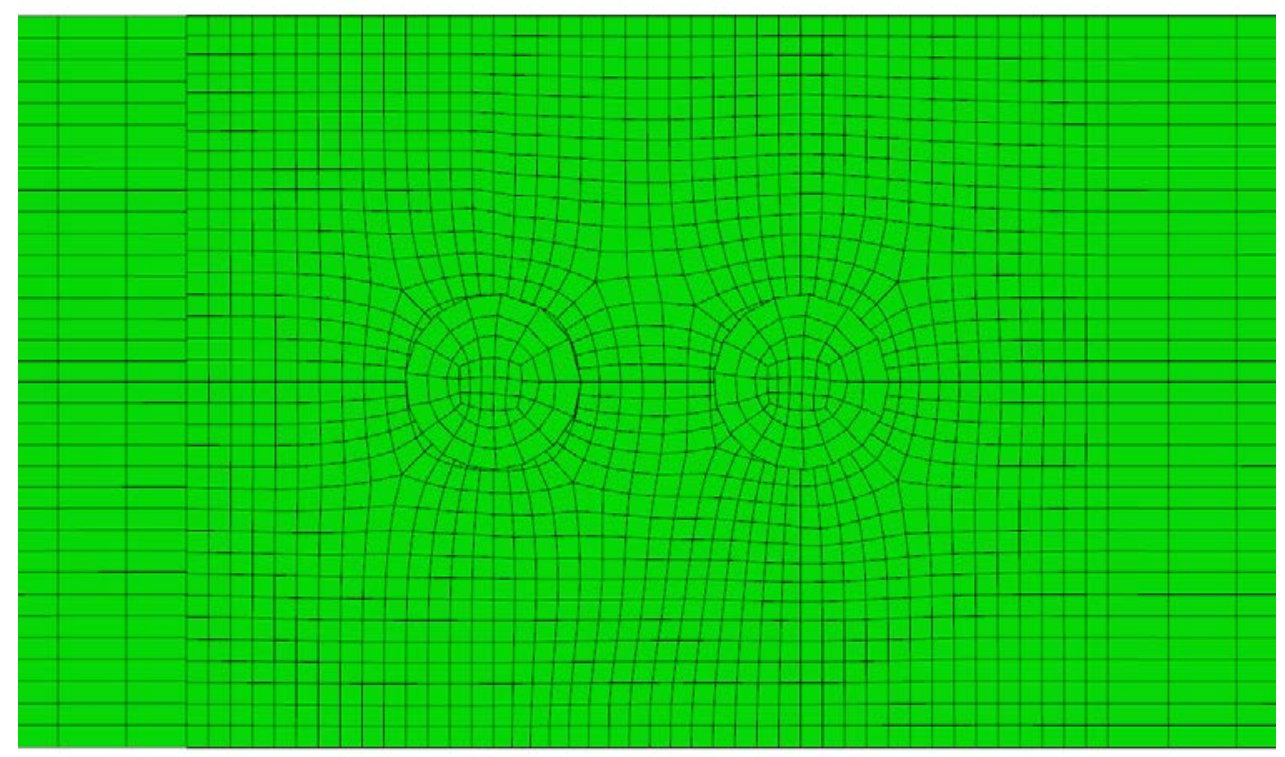

Figure 3-1 Mesh size in overlapped section of specimen 


\subsection{CONSTRAINTS}

The boundary conditions were defined so that the right end, the end of the thicker plate, was fixed. The left end, the end of the thinner plate, was restrained in such a way as to enable the plate to only move in the direction of loading. The test setup of single shear screw connections of thin sheet steels, depicted in Figure 2-50, shows the connection specimen being gripped at the ends. This boundary condition was simulated by restraining the degrees of freedom, using kinematic coupling constraints, of the two reference points (RP1 and RP2), as shown in Figure 3-2.

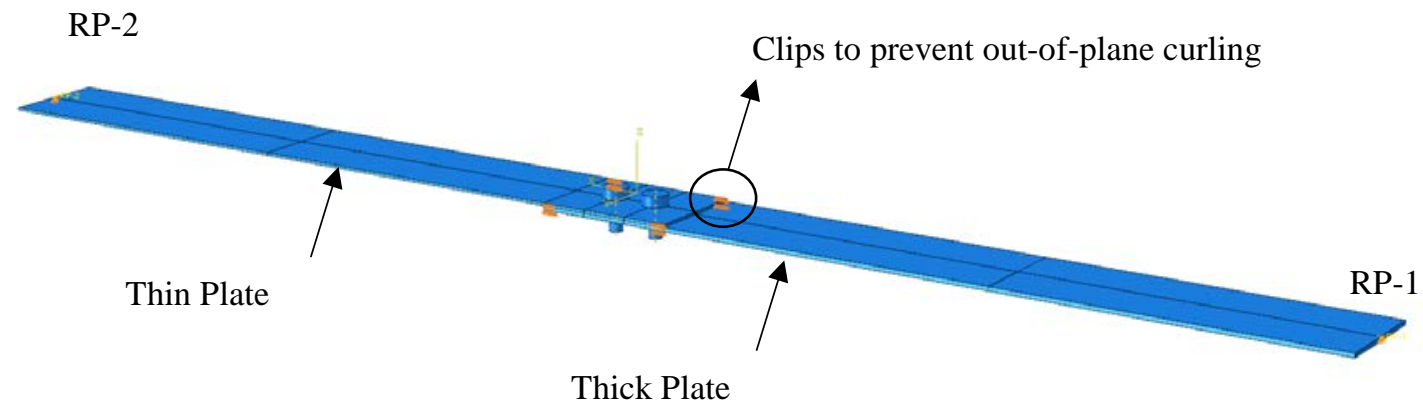

Figure 3-2 Finite element model with boundary conditions

The surface-based coupling constraint provides coupling between a reference node and a group of nodes referred to as the coupling nodes. The kinematic coupling constraints are useful in cases where a large number of nodes are constrained to the rigid body motion of a single node and the degrees of freedom that participate in the constraint are selected individually in a local coordinate system. Kinematic constraints are imposed through the elimination of the degrees of freedom at the coupling nodes. Upon constraint of the displacement degrees of freedom, for any combination, at the coupling nodes, additional displacement constraints; such as multi point constraints, boundary conditions or other kinematic coupling conditions; cannot be applied to any coupling node involved in a kinematic coupling constraint. These same limitations apply for rotational degrees of freedom.

All degrees of freedom were restrained at the reference point RP1, and the translational degree of freedom in the axial direction was released at reference point RP2, where the rest of the degrees of freedom were restrained. Due to the fact that solid elements were used, only axial degrees of freedom could be restrained, and hence the rotational movement remained inactive in this model. In the overlapped part, the nodes in the out-of-plane displacement, at the edges of the plate in a certain width, were restrained in order to simulate the effect of clips, which were used to prevent the extent of out-of-plane curling at the ends of the steel plate. 
The dominant mode of failure which was investigated in this study was through bearing/tilting. Rogers \& Hancock (1998) explained that it is possible to misinterpret failure modes in thin sheet steel bolted connections if curling was not prevented, due to the possible onset of early fracture of the net section. Utilising clips also ensured that the fasteners tilted, more closely mimicking the bearing/tilting mode of failure.

\subsubsection{Loading Speed and Mass Scaling}

The explicit dynamic analysis has low efficiency in calculation procedure compared with that of a conventional static analysis. The computational time involved in running a simulation using explicit time integration is proportional to the time period of the event. The computational cost in a quasi-static analysis can be reduced through either speeding up the simulation or using mass scaling.

Mass scaling enables the analysis to be performed economically, without artificially increasing the rate of loading. The following equations exhibit how the stable time increment is related to the material density. The stability limit for the model is the minimum stable time increment of all elements, and can be expressed as:

$$
\Delta t=L^{e} / c_{d}
$$

Where $L^{e}$ is the characteristic element length and $c_{d}$ is the dilatational wave speed of the material. The dilatational wave speed for a linear elastic material with Poisson's ratio equal to zero is given by

$$
c_{d}=\sqrt{\frac{E}{p}}
$$

Where $\mathrm{E}$ is the Young's Modulus of the material and $p$ is the density. According to the above equations, artificially increasing the material density, by a factor of $f^{2}$ decreases the wave speed by a factor of $f$ and increases the stable time increment by a factor of $f$. When the global stability limit (the time required to propagate a dilatational wave across the smallest element dimension) is increased, fewer increments are required to perform the same analysis, which is the goal of mass scaling. A mass scaling factor of 100, along with a loading rate of 1 $\mathrm{mm} / \mathrm{s}$, was used to accelerate the calculations, as recommended by Yan (2012).

\subsubsection{Loading Method}

The actual time taken for a physical process to occur is referred to as its natural time. In general, it is safe to assume that performing an analysis in the natural time for a quasi-static process, will produce the most accurate static results. If the real-life event occurs in a natural time scale, in which the velocities are zero at conclusion, a dynamic analysis should be able to capture the fact that the analysis has achieved steady state. As long as the solution remains nearly the same as the true static solution, and the dynamic effects remain insignificant, the loading rate can be increased in order to obtain the same physical event in less time. 
Loading was applied by means of the displacement controlled loading method. The tensile axial load was applied through specification of the longitudinal axial displacement to RP2, by means of a prescribed amplitude over the specified time interval. The displacement boundary condition was enforced in an incremental manner, by means of an amplitude curve. Variation of the amplitude with time can be specified in a number of ways, and in this model the type of amplitude influenced the load-deflection behaviour of the screw connection.

In order to ensure accuracy and efficiency, the application of loading in a quasi-static analysis must be as smooth as possible. Sudden spikes cause the propagation of stress waves, inducing noisy or inaccurate solutions. In order to apply the load as smoothly as possible, the acceleration changes must be as small as possible from one increment to the next. If the acceleration is smooth, the changes in velocity and displacement will also be smooth.

In a quasi-static simulation, the work applied by all external forces is nearly equal to the internal energy of the system. The viscously dissipated energy is generally small, unless viscoelastic materials or material damping is used. The inertial forces in a quasi-static analysis could be considered negligible when the velocity of the material in the model is very small. As a general rule, the kinetic energy of the deforming material should not exceed a small fraction, typically between $5 \%$ to $10 \%$ of the total internal energy throughout most of the analysis (ABAQUS, 2015).

\subsubsection{Amplitude}

The smooth step amplitude, connects each data pair of displacements with curves whose first and second derivatives are smooth and whose slopes are zero at each of the data points. Since both of these derivatives are smooth, a displacement loading could be applied with this smooth step using only the initial and final data points, and the intervening motion will be smooth. Using this type of loading amplitude allows one to perform a quasi-static analysis without generating waves due to discontinuity in the rate of applied loading, an example of a smooth amplitude, is given in Figure 3-3.

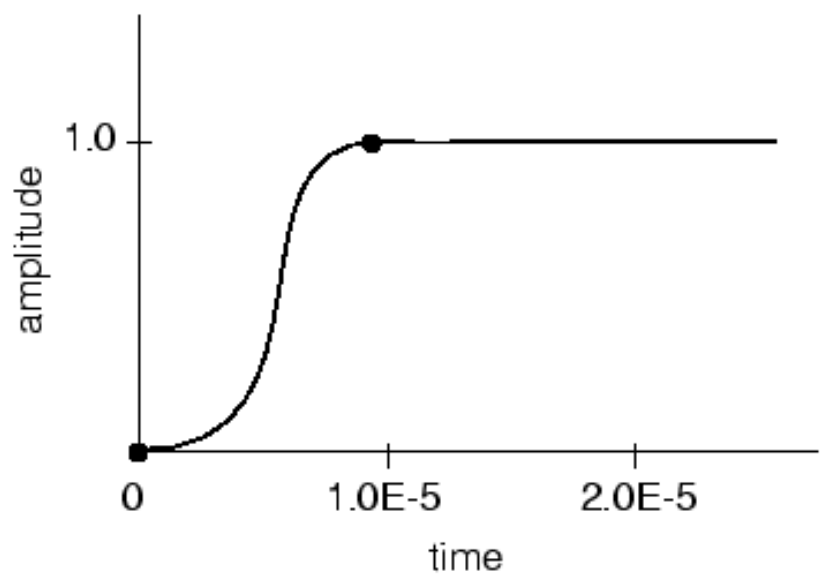

Figure 3-3 Example of an amplitude definition using a smooth step (ABAQUS, 2015) 
Utilising this smooth amplitude did have an effect on the load-deflection behaviour of the model, where the shape of the load deflection curve mimics the shape of the amplitude curve, as depicted in Figure 3-4. Examining the ratio between the kinetic energy and the internal energy reveals that, for the majority of the analysis, the energy ratio was very low (close to zero). However, a spike in the ratio is present in the first few milliseconds, hence applying a tabular amplitude with initial smoothing presents a solution whereby for a specified fraction of the time interval, the tabular curve is replaced by a smooth quadratic time variation. When an amplitude curve is utilised to prescribe a variable in the model, such as a boundary condition, the first and second time derivatives of the variable may also be required.

When a displacement time history is defined by a piecewise linear amplitude variation, the corresponding velocity is piecewise constant and the acceleration may be infinite at the end of each time interval. This behaviour is unrealistic (ABAQUS, 2015), and one can modify the piecewise linear displacement variation into a combination of piecewise linear and piecewise quadratic variations through smoothing. Smoothing ensured that the velocity varied continuously during the time period of the amplitude definition. In order to apply smoothing to an amplitude curve, one can specify the fraction of time interval before and after each time point during which the piecewise linear time variation was replaced by a smooth quadratic time variation. A value of 0.05 was suggested by ABAQUS (2015) and hence applied. This allowed ratio between the kinetic and internal energy not to exceed $5 \%$, as shown in Figure 3-5.

\section{Effect of Loading Amplitude}

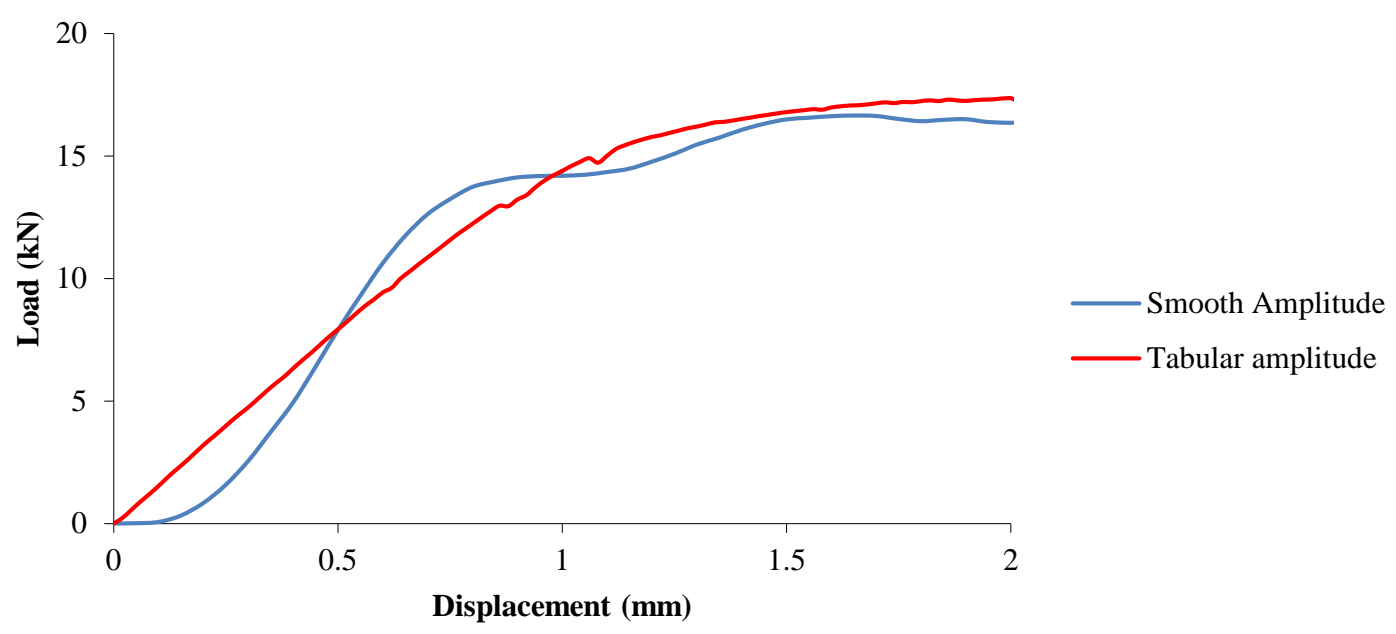

Figure 3-4 Effect of loading amplitude on load displacement curve 


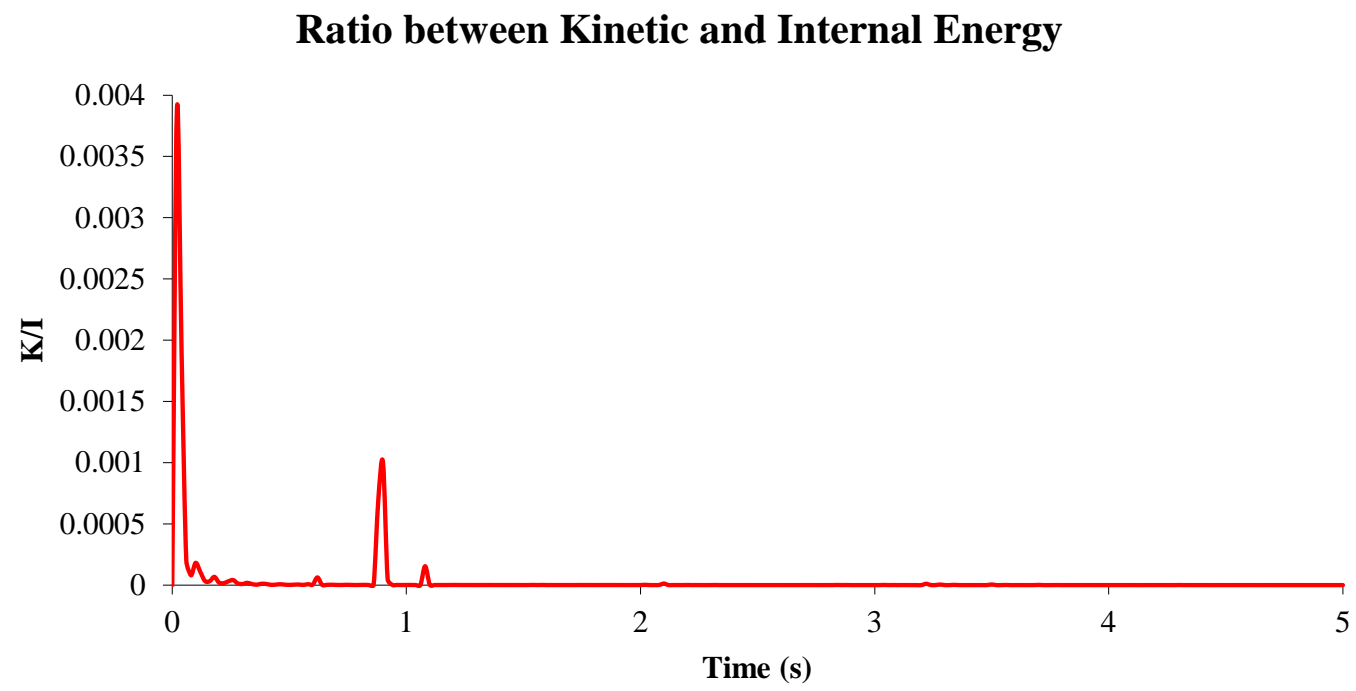

Figure 3-5 Ratio between kinetic and internal energy for model

\subsection{MATERIAL MODELLING}

Most materials utilised in engineering initially respond elastically. Elastic behaviour entails that the deformation is fully recoverable: when the load is removed, the sample returns to its original shape. Once the load exceeds the limits of the material (the "yield load"), the deformation is no longer recoverable and some part of the deformation will remain when the load is removed. Plasticity theories model the mechanical response of the material as it is subjected to non-recoverable deformation in a ductile manner. These theories have been most comprehensively developed for metals.

Most plasticity models in ABAQUS (2015) are "incremental" theories, whereby the mechanical strain rate is divided into an elastic and an inelastic (plastic) component. Incremental plasticity models are generally formulated in terms of:

- A yield surface, generalising the concept of "yield load" into a test function used to determine if the material responds purely elastically at a particular state of stress;

- A flow rule, defining the inelastic deformation that occurs once the material no longer responds purely elastically; and

- Evolution laws defining the material hardening - the method in which the yield and/or flow definitions change as inelastic deformation occurs.

When conducting elastic-plastic analyses at finite strains, it is assumed that the elastic strains are small and the plastic strains dominate the deformation. This restriction is imposed by the elasticity models that ABAQUS utilises. This is justified due to most materials possessing a well-defined yield point that is a very small percentage of their Young's modulus, for example; the yield stress of metals is normally less than $1 \%$ of the Young's modulus of that 
material. Therefore, the elastic strain would also be less than $1 \%$, and the elastic response of the material can be accurately modelled as being linear.

At relatively low temperatures, the yield and inelastic flow of a metal can be typically described with the classical metal plasticity models, provided that the loading is relatively monotonic and creep effects are unimportant. In ABAQUS (2015), these models utilise standard Hill or Mises yield surfaces with associated plastic flow. Perfect plasticity and isotropic hardening definitions are both available in the classical metal plasticity models. Common applications for these include; metal forming, crash analyses and general collapse studies, as the models are simple and adequate for such situations.

ABAQUS (2015) utilises associated plastic flow. Thus, as the material yields, the inelastic deformation rate is in the direction of the normal to the yield surface (the plastic deformation is volume invariant), as shown in Figure 3-6. This assumption is typically acceptable for most calculations involving metals; though this is inappropriate for the detailed analysis of the localisation of plastic flow in sheets of metal, due to the sheet developing texture and eventually tearing apart. Provided the minutiae of these effects are unimportant (or that they could be inferred from less detailed criteria, including reaching a forming limit defined in terms of strain), the associated flow models used with the smooth Mises or Hill yield surfaces usually predict the behaviour with sufficient accuracy.

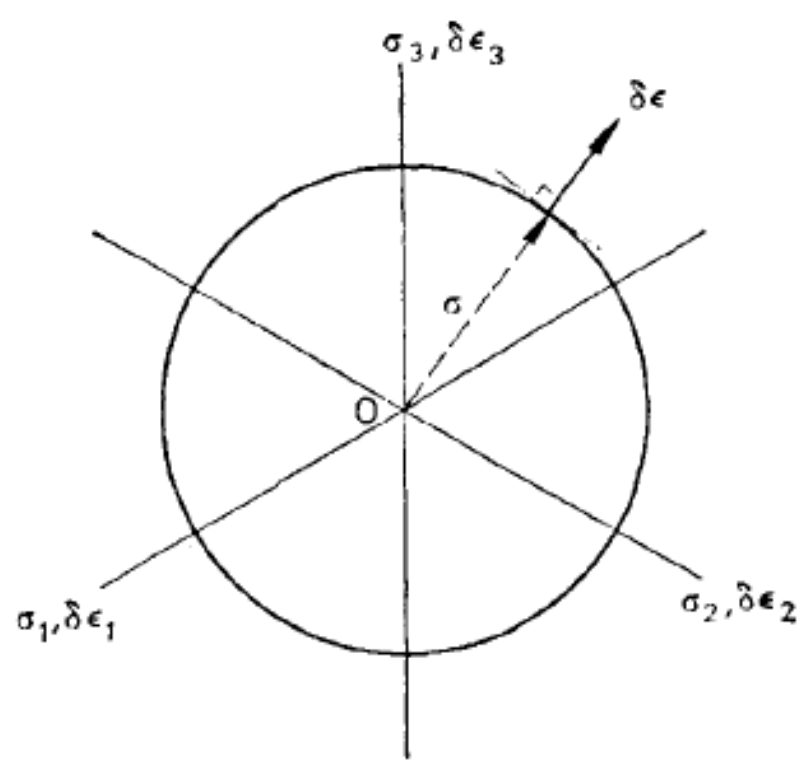

Figure 3-6 Mises yield surface (plan view of $\pi$-plane) and associate strain-increment vector (Calladine, 2011)

The Mises and Hill yield surfaces estimate that yielding of the metal is independent of the equivalent pressure stress (Mac Donald, 2011). This has been experimentally confirmed for most metals, barring voided metals, under positive pressure stress, though this may be inaccurate for metals subjected to high triaxial tension; when the voids may nucleate and propagate within the material. These conditions may arise in stress fields near crack tips, as well as under extreme thermal loading situations which occur during welding processes (ABAQUS, 2015). The Mises yield surface defines isotropic yielding, where the Hill yield 
surface provides for anisotropic yielding. In this model, it was decided to adopt the Mises yield surface with isotropic hardening.

Isotropic hardening can be defined as the yield surface changing in size uniformly in all directions, such that the yield stress increases, or decreases, in all stress directions as plastic straining occurs (Mac Donald, 2011). This is useful in cases involving gross plastic straining, or in situations where the straining at each point is essentially in the same direction in strain space throughout the analysis. Although this is referred to as a "hardening" model, strain softening can also be included.

An isotropic elasto-plastic material model is commonly utilised for metal plasticity modelling, as either a rate-dependent or rate-independent model, and has a particularly simple form (ABAQUS, 2015). Due to this simplicity, the algebraic equations associated with integrating the model can be straightforwardly developed in terms of a single variable, allowing the material stiffness matrix to be written explicitly. The result of this is a particularly efficient code. If isotropic hardening is defined, the yield stress can be obtained as a tabular function of the plastic strain. The yield stress at a given state is then interpolated from the table of data, and remains constant for plastic strains exceeding the last given value as tabular data.

The mechanical properties of the thin sheet steels used in this analysis is provided in Table $3-1$, where the initial elastic modulus $(E), 0.2 \%$ proof stress $\left(f_{0.2}\right)$, tensile strength $\left(f_{u}\right)$, ultimate strain $\left(\varepsilon_{u}\right)$ and maximum strain after fracture $\left(\varepsilon_{f}\right)$ based on a gauge length of $25 \mathrm{~mm}$ are shown.

Table 3-1 Material properties of thin sheet steels (Yan, 2012; Yan \& Young, 2011)

\begin{tabular}{|c|c|c|c|c|c|}
\hline Series & $\boldsymbol{E} \mathbf{( G P a )}$ & $\boldsymbol{f}_{\mathbf{0 . 2}} \mathbf{( M P a )}$ & $\boldsymbol{f}_{\boldsymbol{u}} \mathbf{( M P a )}$ & $\boldsymbol{\varepsilon}_{\boldsymbol{u}} \mathbf{( \% )}$ & $\boldsymbol{\varepsilon}_{\boldsymbol{f}} \mathbf{( \% )}$ \\
\hline $\mathrm{G} 5001.20 \mathrm{~mm}$ & 215 & 613.7 & 627.3 & 4.5 & 9 \\
\hline $\mathrm{G} 4501.90 \mathrm{~mm}$ & 213 & 504.5 & 543.0 & 7.0 & 13.1 \\
\hline
\end{tabular}

The nominal stress - nominal strain $\left(\sigma_{n}-\varepsilon_{n}\right)$ curve obtained from conventional coupon tests do not provide a true indication of the deformation characteristics of a material at higher strain; due to being based entirely on the original dimensions of the specimens; whilst in actuality, the dimensions of the material change continuously during coupon tests. Therefore, the definitions of nominal stress, $F / A_{0}$, and nominal strain, $\Delta l / l_{0}$, where the subscript 0 indicates a value from the undeformed state of the material, must be replaced by new measures (true stress and true strain) of stress and strain, which account for these changes in dimension. In this model, the engineering (nominal) stresses $\left(\sigma_{n}\right)$, and corresponding strains $\left(\varepsilon_{n}\right)$, have been converted into the true stresses $\left(\sigma_{\text {true }}\right)$ and true plastic strains $\left(\varepsilon_{\text {true }}^{p l}\right)$ through the use of the following equations:

$$
\sigma_{\text {true }}=\sigma_{n}\left(1+\varepsilon_{n}\right)
$$




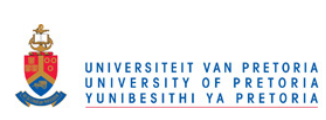

$$
\varepsilon_{\text {true }}^{p l}=\ln \left(1+\varepsilon_{n}\right)-\frac{\sigma_{\text {true }}}{E}
$$

Where $E$ is the initial Young's modulus of the engineering stress-strain curve. Plastic strain values, not total strain values, are used in order to define the hardening behaviour. Furthermore, the first data pair must correspond with the onset of plasticity (the plastic strain value must be zero in the first pair).

\subsection{INTERACTION}

Many engineering problems involve contact between two or more components. In these problems a force normal to the contacting surface acts on the two bodies when they contact each other. If friction is defined between the two surfaces, shear forces resisting the tangential motion (sliding) of the bodies may develop. In a finite element analysis, the contact conditions are a special class of discontinuous constraint, allowing these forces to be transmitted from one part of the model to another. This constraint is discontinuous due to it only being applied when the two surfaces are in contact. When the surfaces separate, no such constraint is applied.

Surface-based contact can utilise either of two algorithms; the general ("automatic") contact algorithm or the contact pair algorithm. The general contact algorithm allows for a highly automatic contact definition, based upon an automatically generated all-inclusive surface definition. Conversely, the contact pair algorithm requires the explicit pairing of surfaces that may potentially come into contact (ABAQUS, 2015).

Either approach comes with its own unique advantages and limitations. Defining a surface based contact simulation, utilising either contact pairs or general contact, requires the following specifications:

- Surface definitions for the bodies that could potentially be in contact;

- The surfaces that interact with one another (the contact interactions);

- Any non-default surface properties to be considered in the contact interactions;

- The mechanical and thermal contact property models, such as the pressureoverclosure relationship, the friction coefficient; and

- Any algorithmic contact controls for the analysis.

The various interfaces in this model were simulated using contact pairs. The contact formulation for the contact pair algorithm in ABAQUS/Explicit includes:

- The contact surface weighting (balanced or pure master-slave);

- The constraint enforcement method (kinematic or penalty); and

- The sliding formulation (finite or small).

When "hard" contact is used, a dramatic change in the contact pressure occurs as the contact conditions change from "open” (a positive clearance) to "closed" (clearance equal to zero). This occasionally makes it difficult for a general static analysis to complete the contact 
simulation. The same could not be said for the explicit method, as iteration is not required. In addition to determining whether contact has occurred at a particular point, the analysis must also calculate the relative sliding of the two surfaces. This calculation can be exceedingly complex, necessitating a distinction be made between analyses where the magnitude of sliding is small, and those where the magnitude of sliding may be infinite. The term "small sliding" is difficult to define, though a general approximation would be: a point contacting the surface does not slide more than a small fraction of a typical element dimension.

\subsubsection{Contact Constraint Enforcement Method}

The contact pair algorithm uses a default kinematic contact formulation, which achieves precise compliance with the contact conditions through the use of a predictor/corrector method. Initially, the increment proceeds under the assumption that contact does not occur. If there is overclosure at the end of the increment, the acceleration is modified so that a correct configuration is obtained, in which the contact constraints are enforced.

The normal contact constraint for contact pairs can be optionally enforced using the penalty contact method, in order to model some types of contact which the kinematic model cannot. When the penalty contact formulation is utilised, equal and opposite contact forces, with magnitudes equivalent to the penalty stiffness multiplied by the penetration distance, are applied to the master and slave nodes at the penetration points.

The penalty contact algorithm results in less rigorous enforcement of contact constraints, when compared to the kinematic algorithm, though the penalty algorithm does allow for the analysis of more general types of contact. Both methods conserve momentum between the contacting bodies, and the enforcement of the contact conditions can either be by means of a pure, or a balanced, master-slave contact pair. The penalty contact method is ideally suited for very general contact modelling, including the following situations:

- Multiple contacts per node, and

- Contact of surfaces also involved in other types of constraints.

The kinematic contact algorithm stringently enforces the contact constraints whilst conserving momentum. In order to attain these qualities within a discretised model, some energy must be absorbed upon impact. To illustrate this, consider a linear elastic truss, modelled with several elements, impacting a rigid wall. Upon impact, the kinetic energy of the leading node is absorbed by the contact algorithm, whilst a stress wave propagates through the truss, eventually causing the truss to rebound from the wall. After rebounding, the kinetic energy is decreased due to the loss of the contact load's energy upon impact. As the mesh is refined, this energy loss is diminished, as a result of the mass and kinetic energy of the leading node of the truss becoming less significant.

On impact, contact forces can also exert negative external work, since they act over the entire increment in which impact occurs, including the fraction of the increment prior to the impact. The opposing contact forces, which possess equivalent magnitudes, act across different distances, thereby exerting a nonzero net work. The net external work of these forces is negative, and the absolute value of the net external work does not exceed the contact node's kinetic energy loss upon impact. 
When the hard kinematic contact algorithm is used, the impact is plastic, and the kinetic energy of the contacting nodes is lost. This loss is insignificant for a refined mesh, but can become significant if a coarse mesh is used. The penalty contact introduces numerical softening to the contact enforcement, analogous to adding elastic springs to the contact interface, meaning that the algorithm does not dissipate energy upon impact, as the energy stored in the springs is recoverable.

Closer examination of the finite element model revealed that in the overlapping area, shown in Figure 3-7, where the screw head and shaft intersect with the top plate, two different contact conditions are simultaneously enforced. The provision of edge clips; the boundary condition preventing out of plane curling; requires that the contact surface participate in both contact and boundary constraint conditions. However, if boundary constraints are applied to contact nodes, the boundary constraints may override the contact constraints. As this can cause misleading results for kinematic contact, the penalty contact algorithm was used instead.

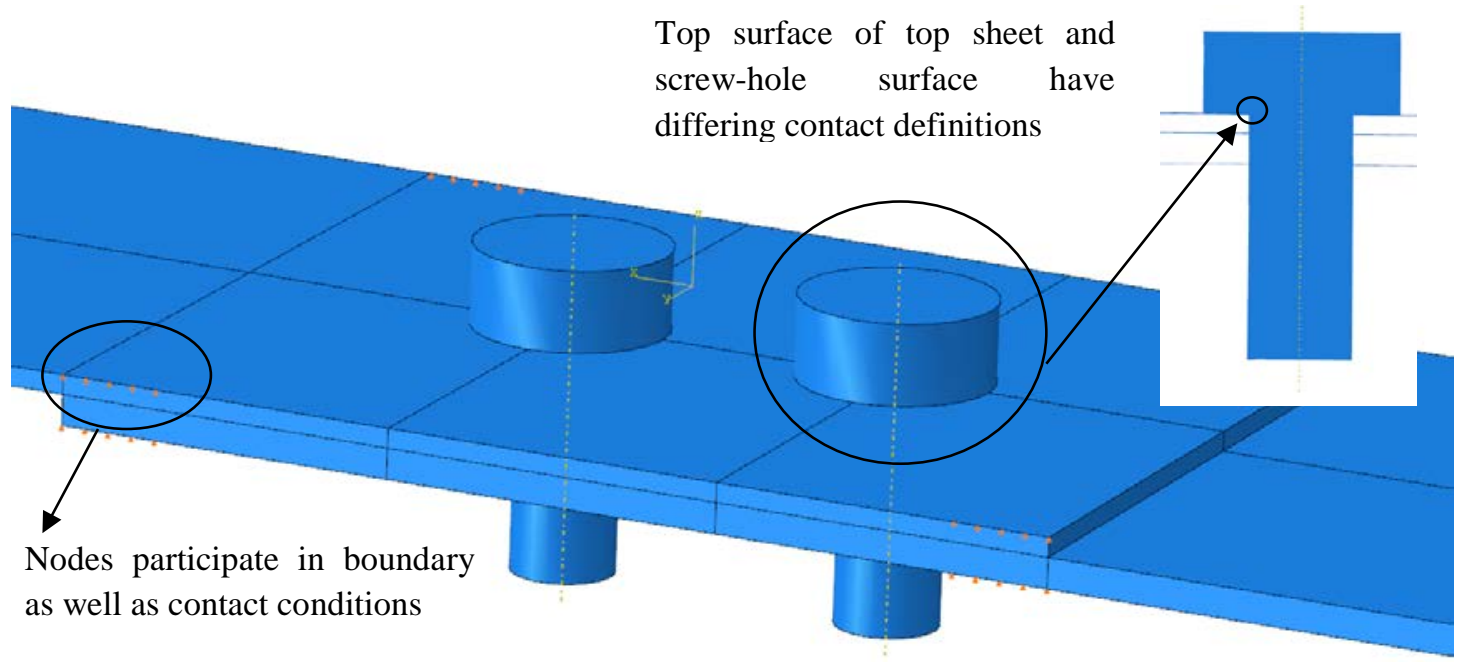

Figure 3-7 Conflicting constraint conditions

\subsubsection{Contact Surface Weighting}

In a pure master-slave approach, one of the surfaces is chosen to be the master surface, whilst the other surface is the slave surface. As the two bodies contact each other, the penetrations are detected, whilst the contact constraints are applied according to the chosen enforcement method, whether kinematic or penalty. Irrespective of the constraint enforcement method, pure master-slave weighting will only resist penetrations of slave nodes into master facets. Penetrations of master nodes into the slave surface can go undetected, unless the mesh on the slave surface is adequately refined, as depicted in Figure 3-8. 


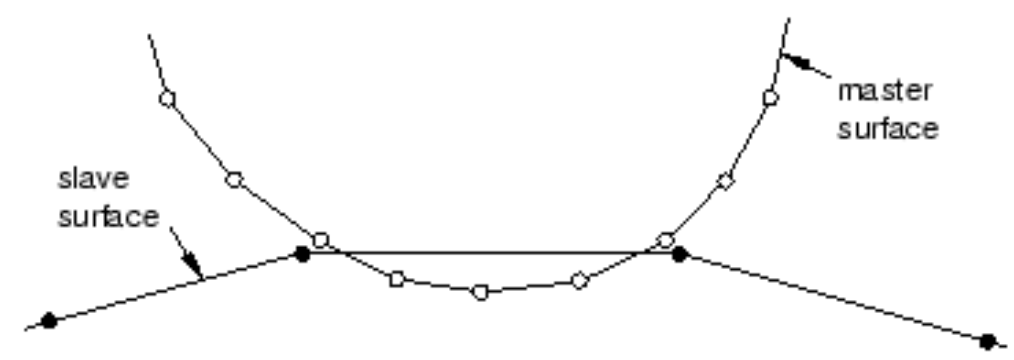

Figure 3-8 Penetration of master nodes into slave surface with pure master-slave contact (ABAQUS, 2015)

The balanced master-slave contact applies the pure master-slave approach twice, reversing the surfaces on the second pass. In essence, one set of contact constraints is obtained with the first surface as the slave, and the next set of constraints is solicited with the second surface acting as the slave. The acceleration force corrections are determined through a weighted average of the two calculations. The balanced master-slave contact constraint, when kinematic compliance is used, is illustrated in Figure 3-9. When utilising the balanced approach, penetration in the contacting bodies is minimised, providing more accurate results in most applications.

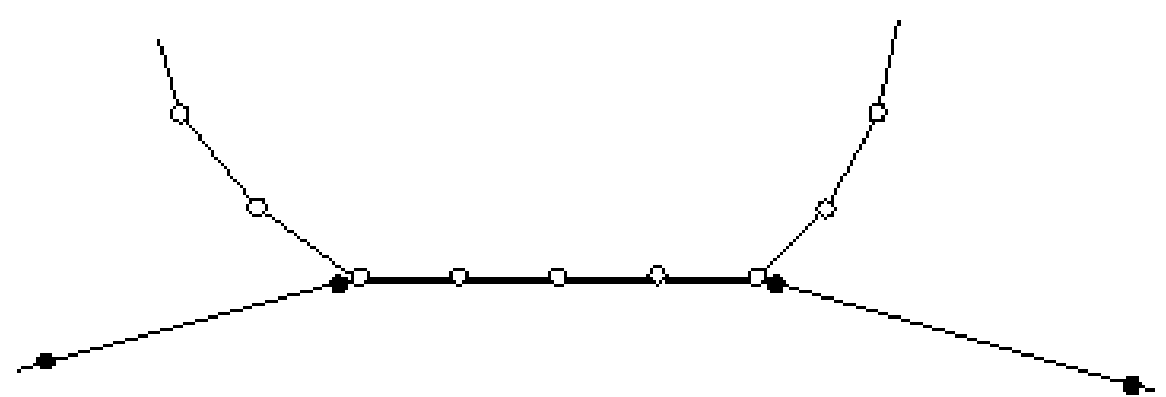

Figure 3-9 Balanced master-slave contact constraint with kinematic compliance (ABAQUS, 2015)

\subsubsection{Energy Considerations}

The energy output is often a significant component of an explicit analysis. Comparisons of the various energy components can help to evaluate whether the analysis is providing an appropriate response. The energy balance of the entire model, according to ABAQUS (2015), can be expressed as:

$$
E_{T O T A L}=E_{I}+E_{V}+E_{F D}+E_{K E}+E_{I H E}-E_{W}-E_{P W}-E_{C W}-E_{M W}-E_{H F}
$$


Where $E_{I}$ is the internal energy, $E_{V}$ is the viscous energy dissipated, $E_{F D}$ is the frictional energy dissipated, $E_{K E}$ is the kinetic energy, $E_{I H E}$ is the internal heat energy, $E_{W}$ is the work done by the externally applied loads, and $E_{P W}, E_{C W}$, and $E_{M W}$ are the work done by the contact penalties, the constraint penalties, and the propelling added mass, respectively. $E_{H F}$ is the external heat energy through external fluxes. The sum of these energy components is $E_{\text {TOTAL}}$, and should be constant.

The decision was made to rather not model the screw thread in this finite model, and instead to simulate the effect of the thread artificially, through adjustment of the frictional coefficient for contact between the plate and the screw shaft which agreed with theories presented by Fan et al. (1997a; 1997b) as well as Yan (2012). Consequently, the energy generated due to the frictional work will not be accurately represented.

Comparison of the kinematic and penalty contact constraint enforcements' total energy output revealed that when kinematic contact enforcement is utilised, the total energy remains zero, though the model deformation was deemed to be unrealistic, as depicted in Figure 3-10, due to the behaviour of the connections observed during the initial experimental phase as well as from the deformed specimen observed in the literature study presented in Chapter 2. The load deflection behaviour was furthermore observed to be substantially lower than the equivalent experimental results.

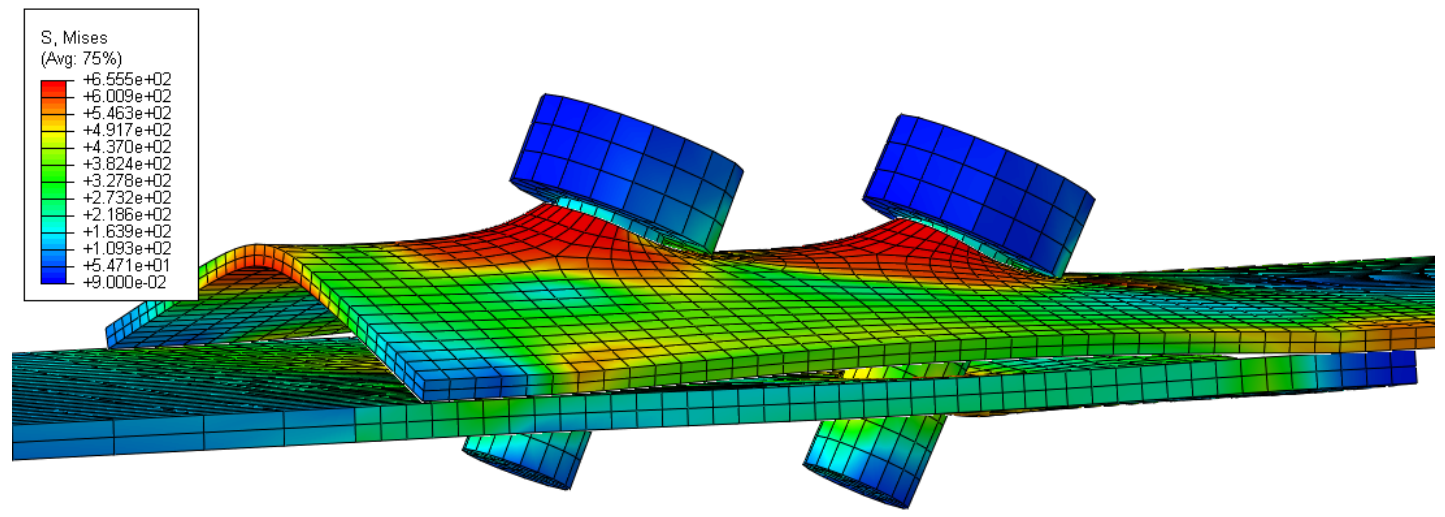

Figure 3-10 Deformation of model with kinematic contact enforcement

It was determined that the penalty contact constraint enforcement provided a higher level of accuracy, both in terms of the load deflection behaviour, as well as the fact that it provided a realistic deformation, as shown in Figure 3-11, although the total energy did not remain constant. This loss in total energy, as can be seen in Figure 3-12, could be attributed to the contact energy, as a result of the penalty contact constraint. 


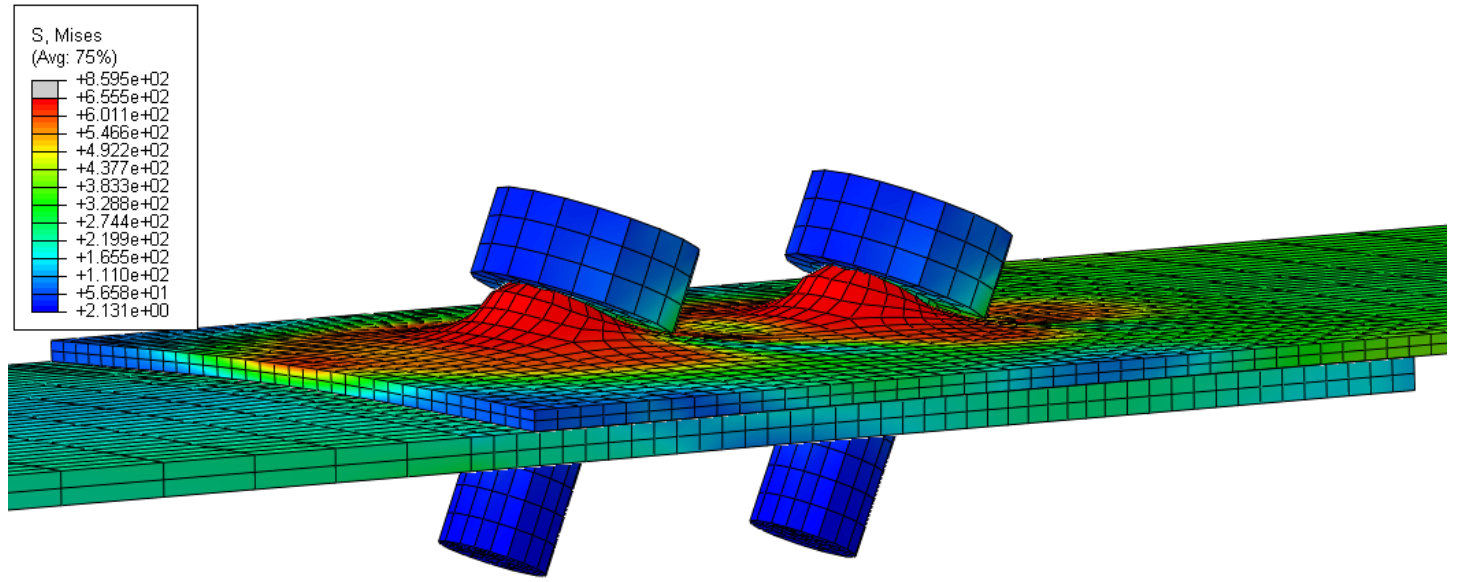

Figure 3-11 Deformation of model with penalty contact enforcement

\section{Loss in total energy}

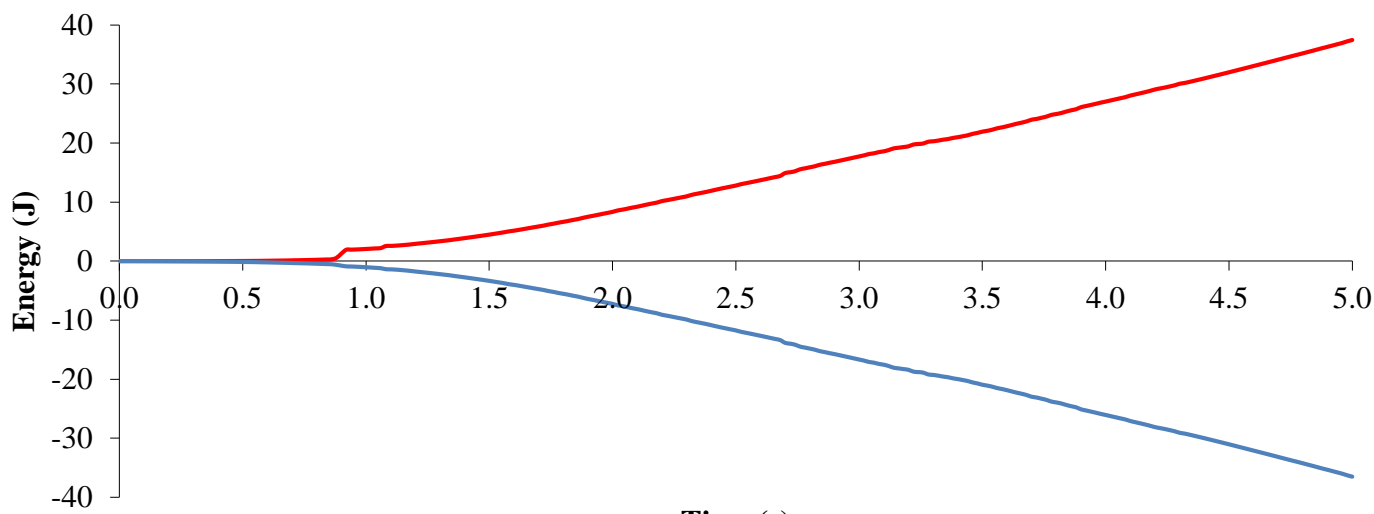

Time (s)

— Contact Energy due to Penalty Constraint ——Total Energy

Figure 3-12 Loss in total energy accounted for in contact energy due to penalty contact constraint

Utilising a pure master-slave contact setting, with the weight of the master surface set to 1 , decreased the loss in total energy, but also resulted in excessive penetrations into the slave surfaces, as shown in Figure 3-13. The decision was made to not place too much emphasis on the total energy, due to the fact that the effect of the screw thread was artificially modelled, resulting in the creation of artificial energies. The reasons for this are most likely due to; the model not accounting for the effect of heat generation, as a result of the increase in the friction coefficient; the lack of a fully coupled temperature-displacement analysis, as well as not accounting for the adiabatic heating effects. 


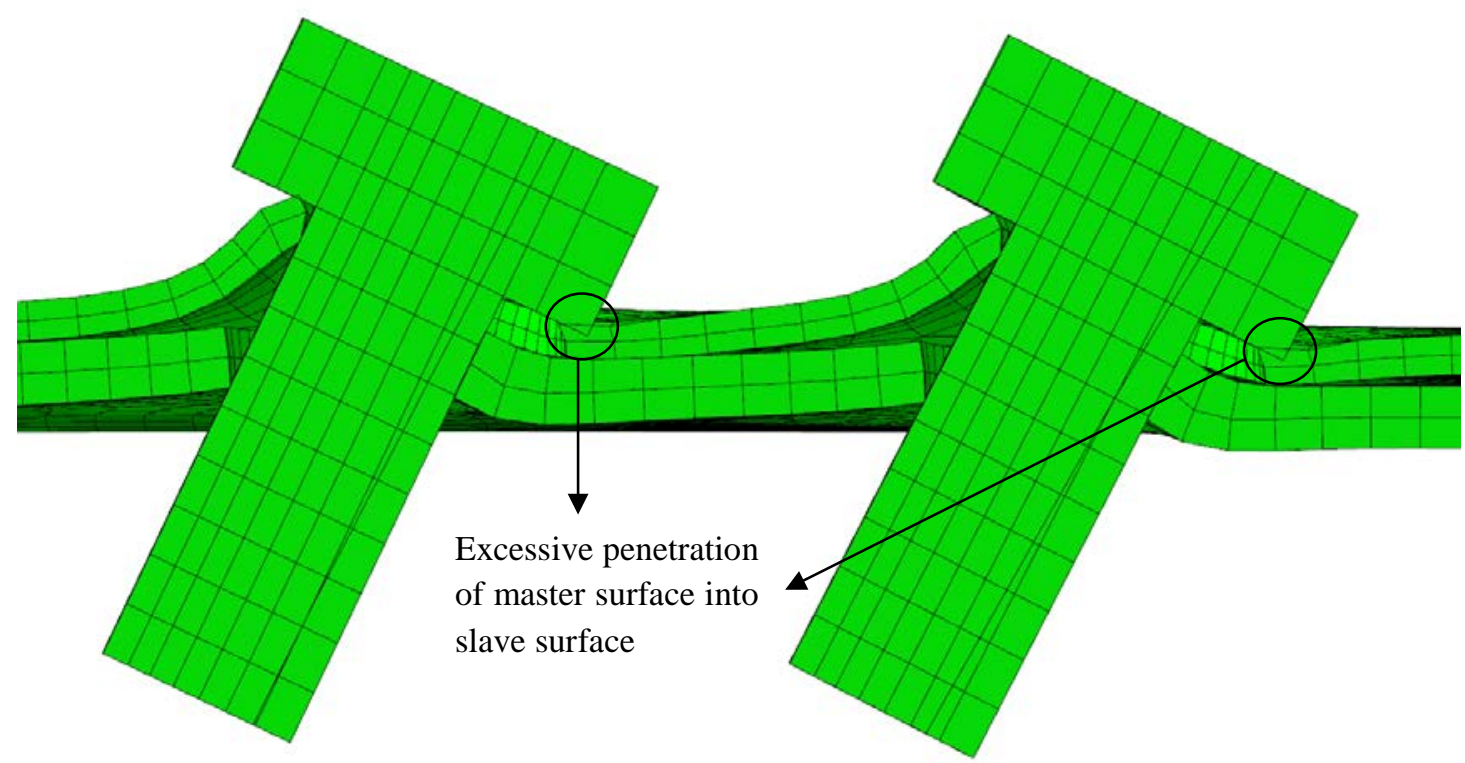

Figure 3-13 Excessive penetrations of master surface into slave surface

\subsubsection{Contact Property Models}

Contact interactions in a model can refer to a contact property definition, akin to how elements refer to an element property definition. When surfaces contact, they usually transmit shear, as well as normal, forces across their interface. Generally, there is a relationship between these two force components, known as friction between the contacting bodies, and is usually expressed in terms of these stresses at the interface of the bodies. The available friction models include the classical isotropic friction model, which in its general form allows for the friction coefficient to be defined.

The Coulomb friction model relates the maximum allowable frictional (shear) stress across an interface, to the contact pressure between the contacting bodies. In its basic form, the Coulomb friction model carries shear stresses, up to a certain magnitude, across the interface of two contacting surfaces before they start sliding across each other; a phenomenon known as sticking. The Coulomb friction model defines this critical shear stress, where the surfaces start to slide across each other, as a fraction of the contact pressure between those surfaces. The stick/slip calculations determine when a point transitions from sticking to slipping, and this fraction is known as the coefficient of friction.

The basic friction model assumes that the coefficient of friction, $\mu$, is identical in all directions (isotropic friction). In three-dimensional simulations, there are two orthogonal components of shear stress, $\tau_{1}$ and $\tau_{2}$, acting in the local tangential directions for the contact surfaces. ABAQUS combines these two components into an "equivalent shear stress" for the purposes of the stick/slip calculations. Furthermore, the two slip velocity components are also combined into an "equivalent slip rate". The stick/slip calculations define a surface, in the contact pressure-shear stress space, along which a point transitions from sticking to slipping. A two-dimensional representation of this is illustrated in Figure 3-14. 


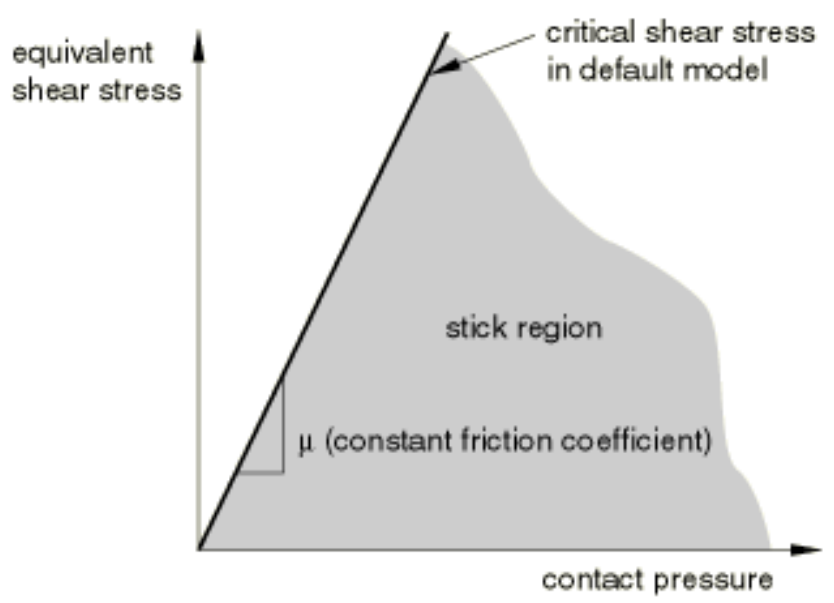

Figure 3-14 Slip regions for the basic coulomb friction model (ABAQUS, 2015)

In this simulation, the choice of an appropriate friction model was guided through taking cognisance of the following principles:

- Closest to reality;

- If the concerned part of the structure was simplified in the simulation, the choice should be made in a way that the simplified structure better simulate the real structure than with a coefficient closer to reality.

The contact laws utilised for the interfaces are provided in Table 3-2. As the screw threads were not modelled, a coefficient of friction, $(\mu=0.4)$, was included in order to prevent premature sliding of the sheets along the screw shaft, as the screw rotates. The friction coefficients utilised in this study was comparative to those used by Fan et al. (1997a; 1997b).

Table 3-2 Contact Property Models (Yan, 2012)

\begin{tabular}{|l|l|l|}
\hline \multicolumn{1}{|c|}{ Surface 1 } & \multicolumn{1}{|c|}{ Surface 2 } & \multicolumn{1}{c|}{ Friction Coefficient } \\
\hline Screw Shaft & Top Plate & 0.4 \\
\hline Screw Shaft & Bottom Plate & 0.4 \\
\hline Screw Head & Top Plate & Frictionless \\
\hline Bottom Plate & Top Plate & 0.13 \\
\hline
\end{tabular}

\subsection{VERIFICATION OF FINITE ELEMENT MODEL}

For the purposes of the numerical investigation, the verification process entailed the calibration of the finite element model to the work of another researcher. The decision was made to extend the study performed by Yan (2012), for the behaviour of thin sheet steel screw connections, at ambient temperatures only. The geometric dimensions of the finite element model were designed according to the details of the specimens described by Yan (2012), and the displacement controlled loading was applied up to a maximum displacement of $5 \mathrm{~mm}$. 
It should be noted that the propagation of brittle cracks in the vicinity of the screw hole, as would be observed in experimental testing, was not observed during the analysis of the FEM. In general, there are three modes in which crack formation can occur (Figure 3-15):

- Mode I - Failure is related to the crack opening;

- Mode II - Failure in sliding is a pure shear failure mode;

- $\quad$ Mode III - Failure in tearing can be considered as out-of-plane shearing.

In many applications, such as the sheet metal failure behind the screw, the material would fail in a mixed mode failure. In order to ensure the simplicity of the modelling procedure, a progressive damage material model for the steel sheets was not employed; a fact which most likely contributed to the absence of brittle crack formation. The reaction force versus the displacement curve generated with this model is presented in Figure 3-16; the jagged shape of the curve is similar to that obtained by Lu et al. (2012), shown in Figure 2-49.

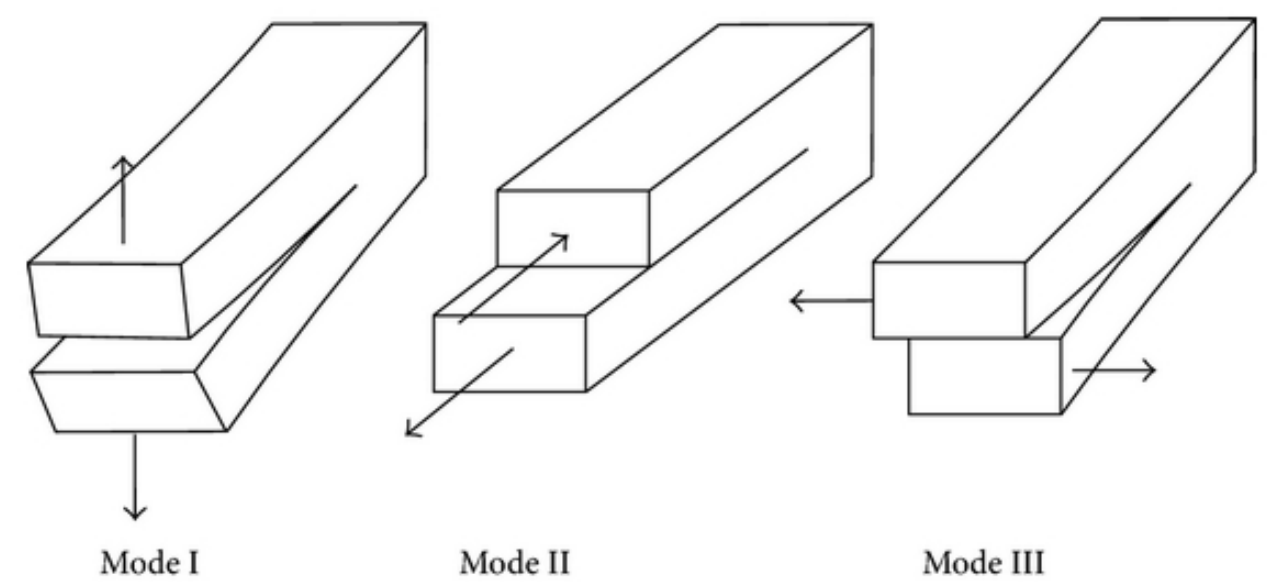

Figure 3-15 Different failure modes of crack propagation (Ugural \& Fenster, 2012)

\section{Reaction Force vs Displacement}

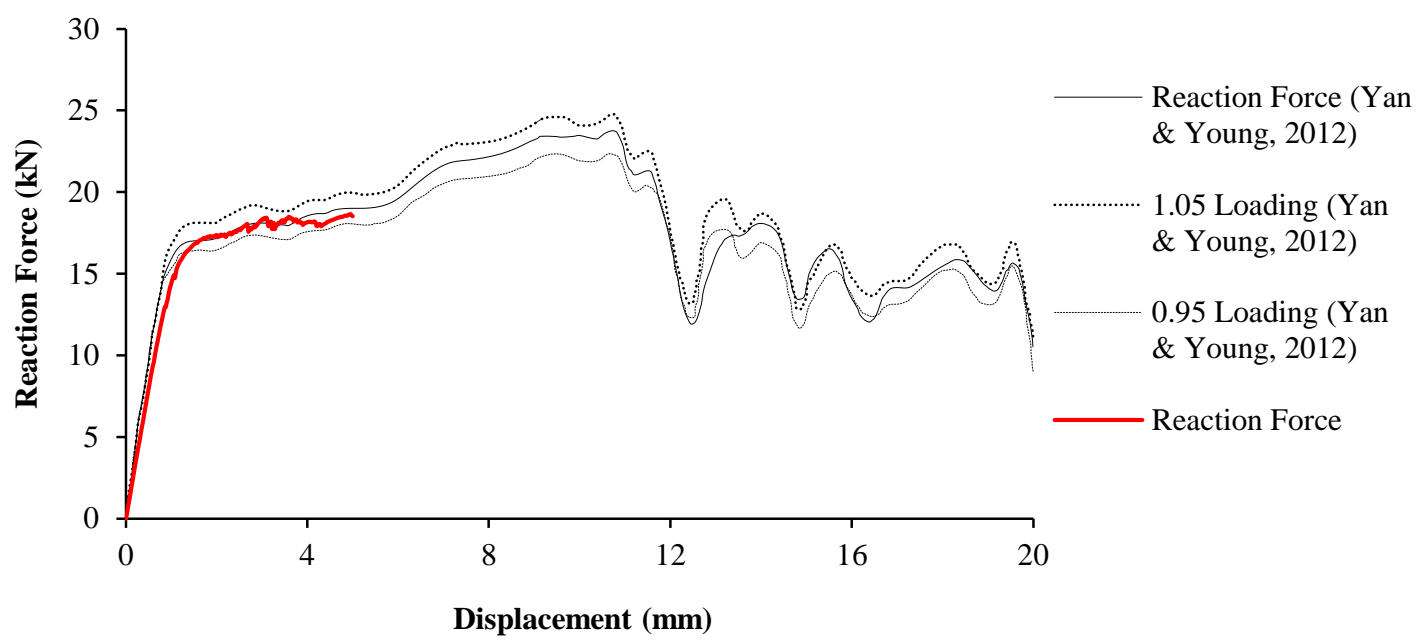

Figure 3-16 FEA curve of reaction force vs displacement 
The reaction force versus displacement curve presented illustrates that the modelling results obtained remains within the $5 \%$ top and bottom curves obtained by Yan (2012). A Von Mises stress plot of the side profile for the connections is shown in Figure 3-17. The observation can be made that, as the load is applied, the screw rotates, and the steel sheet bends up behind the screw; indicating that this follows the archetypical bearing/tilting mode of failure. Upon removal of the screws, in order to inspect the stress plot of the two steel sheets, depicted in Figure $3-18$, it can be seen that the screw hole is enlarged, again substantiating the bearing/tilting failure mode.

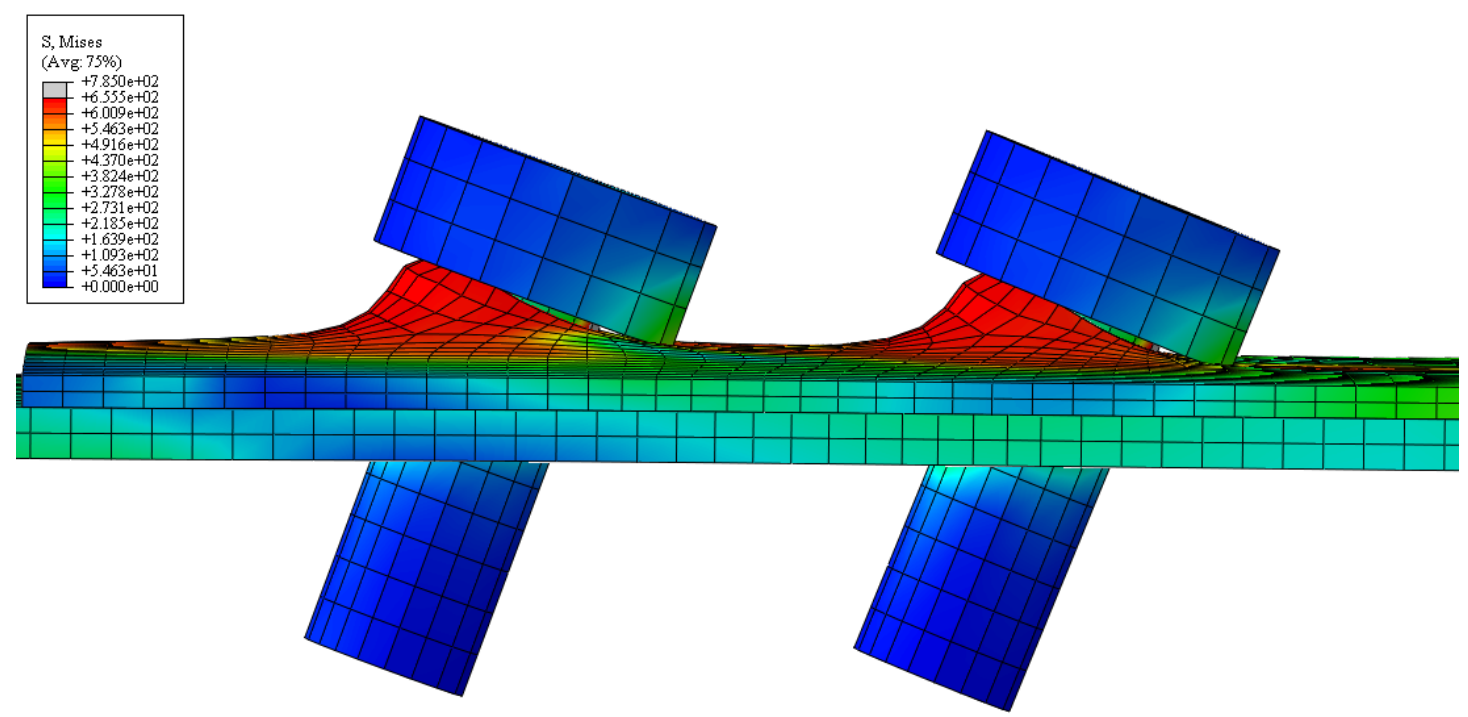

Figure 3-17 Side profile of deformed model with Von Mises stress plot

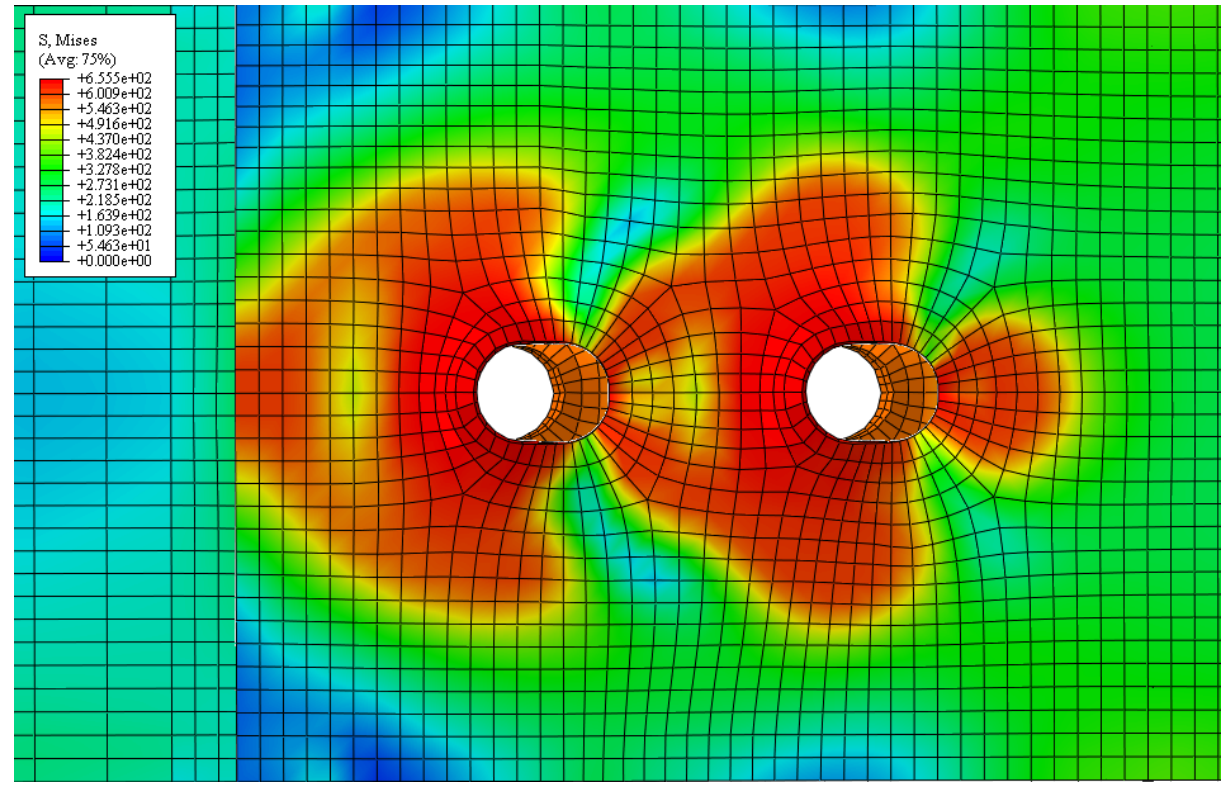

Figure 3-18 Top view of deformed model with Von Mises stress plot 
In order to obtain verification of the mode of failure of the connection, the equivalent plastic strain (PEEQ) can be used to examine the area where the material is yielding. In isotropic hardening plasticity theory, the PEEQ is defined by ABAQUS (2015) as the total accumulation of plastic strain to define the yield surface size and can be described by the equation:

$$
P E E Q=\int_{0}^{t} \bar{\varepsilon}^{p l} d t
$$

where for classical metal (Mises) plasticity $\bar{\varepsilon}^{p l}=\sqrt{\frac{2}{3} \dot{\varepsilon}^{p l}: \dot{\varepsilon}^{p l}}$. The “:” operator indicates the Matrix or Frobenius Scalar product given by $A: B=\operatorname{trace}\left(A^{T} B\right)$. A view of the deformed model with the PEEQ plot is given in Figure 3-19.

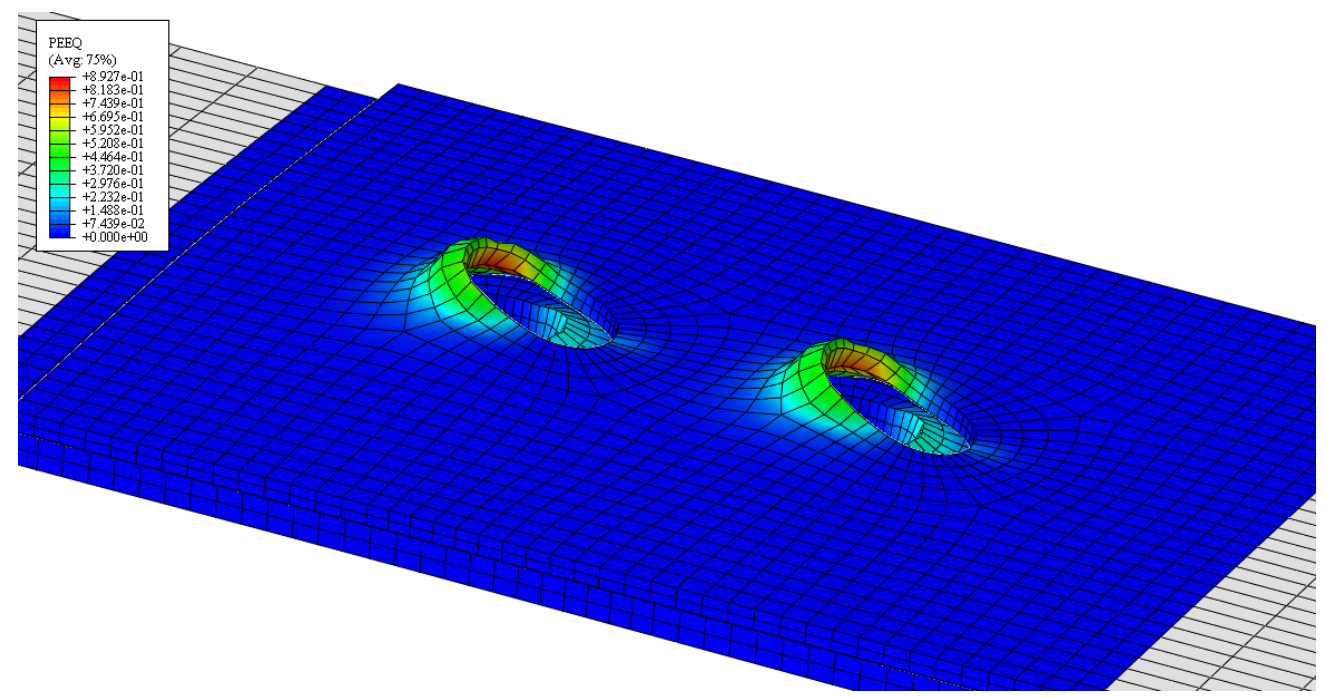

Figure 3-19 View of deformed model with PEEQ plot

\subsection{SUMMARY}

The finite element program ABAQUS V6.13 (2015) was used in the analysis for the simulation of single shear screw connections of thin sheet steels. The four main components were carefully considered in the FEM, the steel plates, the screws, the interfaces between these two components, and the loading method.

The fundamental function of a structural connection is to transfer a load between adjacent components, which, depending on both the type of connection, as well as the applied load, these elements may be subject to; bending, compression, tension, torsion and shear. In the numerical analysis of these elements, the structural assembly is simplified in order to increase the computational efficiency. Even though screw failure was not considered, and the screw 
material was regarded as linear elastic, the screws nonetheless had to be discretised to be involved in the model, in order to ascertain the degree of screw rotation.

The 3D shell element has found widespread use in the modelling of sheeting, but upon examination of the experimental studies, it can be found that in the area below the screw head, the sheet is subjected to transverse compression - a finding which is incompatible with shell hypothesis (Fan et al. (1997a; 1997b)). This finding necessitated the use of C3D8R solid elements, which can be defined as three-dimensional, hexahedral 8-node linear brick elements, with only a single integration point, and hourglass control.

The experimental studies showed that deformation primarily originated from the local area of the steel sheets behind the screw, exhibiting buckling at high loads. This necessitated the refinement of the mesh in this area, but it was noted that the computational cost of using the explicit method was proportional to the number of elements, and inversely proportional to the dimensions of the smallest element. Thus, the average size of the finite element mesh was set at $1.5 \mathrm{~mm}$ in the overlapped part of the connections, with a finer mesh comprising 40 elements in the vicinity of the screw hole. The mesh became gradually coarser towards the end of the specimen, and the steel sheets were two elements thick.

The boundary conditions were defined so that the end of the thicker plate was fixed, and the end of the thinner plate was restrained in such a manner as to only enable it to move in the direction of loading. This was simulated by restraining the degrees of freedom of two reference points through the use of kinematic coupling constraints. Loading was then applied through the use of the displacement controlled loading method, which uses a prescribed amplitude over a specified time interval, which itself was determined by trial and error in order to ensure that the analysis remained in a quasi-static state.

Utilising the smooth step loading amplitude allows for a quasi-static analysis to be performed without the generation of waves, which is due to the discontinuity in the rate of applied loading. In order to ensure that the analysis remains in a quasi-static state, whereby the inertial forces are negligible, the general rule is to ensure that the ratio between the kinetic energy to the internal energy do not exceed $5 \%$ to $10 \%$. Examination of the ratio between the kinetic and internal energy revealed the energy ratio to be close to zero for the majority of the analysis, though a spike in the first few milliseconds necessitated the application of a tabular amplitude with initial smoothing to prevent the ratio from exceeding $5 \%$. The explicit dynamic analysis has a higher computational cost than a conventional static analysis, which can be reduced through either the application of mass scaling or speeding up the simulation. In this numerical model, the use of mass scaling was employed.

Plasticity theories have been comprehensively developed for metals, and model the mechanical response of the material as it is subjected to non-recoverable deformation in a ductile manner. The nominal stress - nominal strain curve obtained from conventional coupon tests does not provide a true indication of the deformation characteristics of a material at higher strain, and must therefore be converted into true stress and true plastic strain values.

In a finite element analysis, the contact conditions are a special class of discontinuous constraint, and were simulated in this instance using contact pairs. The screw threads were not modelled, and in order to prevent premature sliding of the sheets along the screw shaft as they rotate, a coefficient of friction, $(\mu=0.4)$, was included. 
The contact pair algorithm in ABAQUS/Explicit uses a default kinematic contact formulation, and a predictor/corrector method, to achieve compliance with the contact conditions. The penalty contact algorithm allows for the analysis of more general types of contact, through less rigorous enforcement of contact constraints. Close examination of the overlapping area of the model showed that two different contact conditions are simultaneously enforced, which necessitated the use of the penalty contact algorithm.

The energy output is a significant component of the explicit analysis, and comparison of the kinematic and penalty contact constraint enforcements' total energy output revealed that; the penalty contact method provided a higher level of accuracy in terms of the load deflection behaviour, along with a realistic simulation of the deformation. However, the total energy did not remain constant, and this loss was attributed to the contact energy as a result of the penalty contact constraint.

The finite element model was verified through calibration against the research conducted by Yan (2012), and the decision was made to limit the application of the displacement controlled loading, up to a maximum of $5 \mathrm{~mm}$. In order to ensure the simplicity of the FE model, a progressive damage material model was not included. The finite element analysis results obtained remained within the $5 \%$ top and bottom curves obtained by Yan (2012)

A Von Mises stress plot of the side profile for the verified model was presented where it was observed that as the load is applied, the screw rotates, and follows the archetypical bearing/tilting mode of failure. This was further verified through examination of the area where the material was yielding using the equivalent plastic strain (PEEQ), which was defined as the total accumulation of plastic strain to define the yield surface size. 


\section{PARAMETRIC STUDY}

\subsection{INTRODUCTION}

The previous chapter illustrated that not only could the calibrated finite element model effectively predict the experimental behaviour in terms of the load-deformation, but also in terms of the mode of failure. This final calibrated model was utilised in the subsequent parametric studies. In order to conduct the parametric study, the determination of the range of input variables was very important. Therefore, the geometric parameters of the model were selected and varied within the comprehensive practical ranges of single shear screw connections. The different parameters, based on common practical details, were selected in order to investigate the effect of the sheet steel thickness, as well as the number, pattern and size of the screws utilised. The objective of the parametric study was to determine whether the finite element model could effectively simulate the expected experimental behaviour of screwed connections in single shear.

The parametric study consisted of a total of 20 specimens, maintaining the same material properties as the calibrated model for the top and bottom steel sheets, as well as the loading, boundary and contact conditions, with all models pulled to a maximum displacement of 5 $\mathrm{mm}$. The specimens were labelled such that the plate thicknesses of the two pieces of thin sheet steels, number of screws, arrangement of screws and screw sizes could be expediently identified. For instance, the label '080-120-S2-P-14' defines the following specimen:

- The first three numbers indicate the thickness of the top steel sheet which is in contact with the screw head $(080=0.8 \mathrm{~mm})$;

- The next three numbers indicate the thickness of the bottom steel sheet which is not in contact with the screw head $(120=1.2 \mathrm{~mm})$;

- The third segment of the label represents the number of screws in the connection (S2 means two screw connections, S4 means 4 screws and so forth);

- The fourth segment indicates the arrangement of the screws:

0 ' $\mathrm{P}$ ' indicates screws arranged parallel to the applied force,

$\mathrm{o}$ ' $\mathrm{V}$ ' indicates that the screws were arranged perpendicular to the applied force,

0 ' $S$ ' indicates the four screw square arrangement, and

o ' $\mathrm{D}$ ' indicates the four screw diamond arrangement;

- The fifth and last part of the label represents the screw gauge number (14 = $6.35 \mathrm{~mm}$ diameter) of the specimen, given in Table 2-1. 


\subsection{COMPARISON OF FEA TO FAILURE CRITERION PREDICTED LOADS}

Results obtained through finite element analysis were compared to the relevant design standards (AISI S100, 2007; AS/NZS 4600, 2005; EC3, 2006). In this analysis the model was only pulled to a maximum displacement of $5 \mathrm{~mm}$, hence the ultimate load for this comparison was defined to be that of a deflection based load, and not the maximum load attainable by the connection $\left(P_{\text {ultimate }}\right)$. As specified in ECCS Recommendations $(1987 ; 2009)$, in shear tests, it is recommended to define the failure load as the peak load at/or before a connection displacement of $3 \mathrm{~mm}\left(P_{3.0-F E A}\right)$. According to the Research council on Structural Connections (2004) and the American Institute of Steel Construction (1989; 2005), there is a limiting equation for when the deformation of the bolt hole at service level is a design consideration. The nominal strength is limited by $2.4 \mathrm{dtF}_{\mathrm{u}}$ and when the hole deformation is not a consideration it is limited by $3 \mathrm{dtF}_{\mathrm{u}}$. The lower value of the $2.4 \mathrm{dtF}_{\mathrm{u}}$ was stated to provide a bearing strength limit state that is attainable at reasonable deformation (1.4 inch) which relates back to $6.35 \mathrm{~mm}$. This deformation requirement was attributed to the fact that tests have demonstrated that, as the bearing stress is increased beyond the limiting bearing resistance values, as determined through equations (2-4) to (2-8), a hole elongation greater than $6.35 \mathrm{~mm}\left(P_{6.35-F E A}\right)$ will begin to develop. Strength and deformation limits were thus jointly used in these equations to evaluate the bearing strength test results for the design equations. The various loads, at their respective connection displacement are shown in Figure 4-1. In this study, the deflection based load was only considered at/or before a connection displacement of $3 \mathrm{~mm}$.

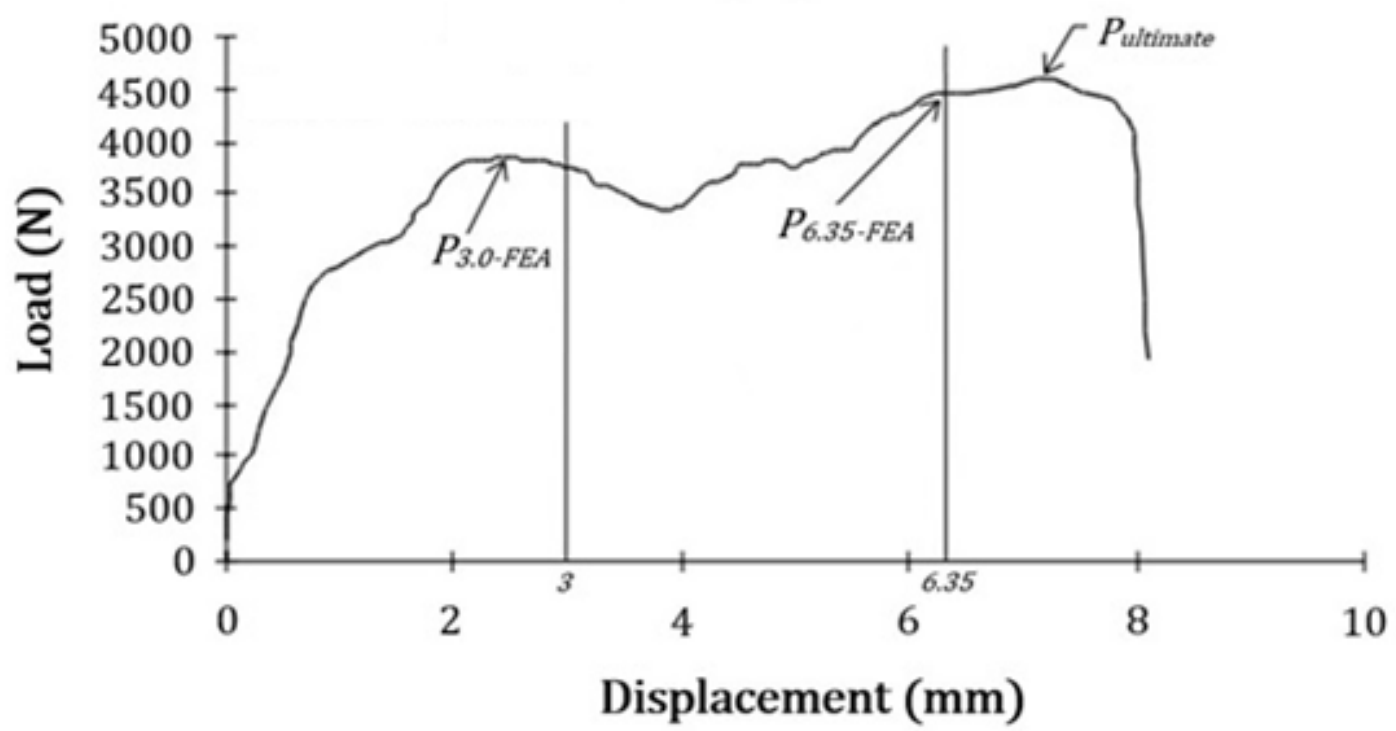

Figure 4-1 Definition of $P_{3.0-F E A}, P_{6.35-F E A}$ and $P_{\text {ultimate }}$ 
As shown in the literature study, presented in Chapter 2, when utilising multiple columns of screws, the column closest to the applied load would experience the highest degree of deformation (Koka et al., 1997). The conjecture could be made that the screw hole closest to the applied load would experience the most deformation. Reference nodes were placed, as shown in Figure 4-2, at the edges of the screw holes of the top and bottom plates. The difference between the displacements of these two nodes would thus be a conservative estimate of the displacement of the connection. Hence, deflection based load was defined as the maximum load at/or before a screw hole deformation of $3 \mathrm{~mm}$.

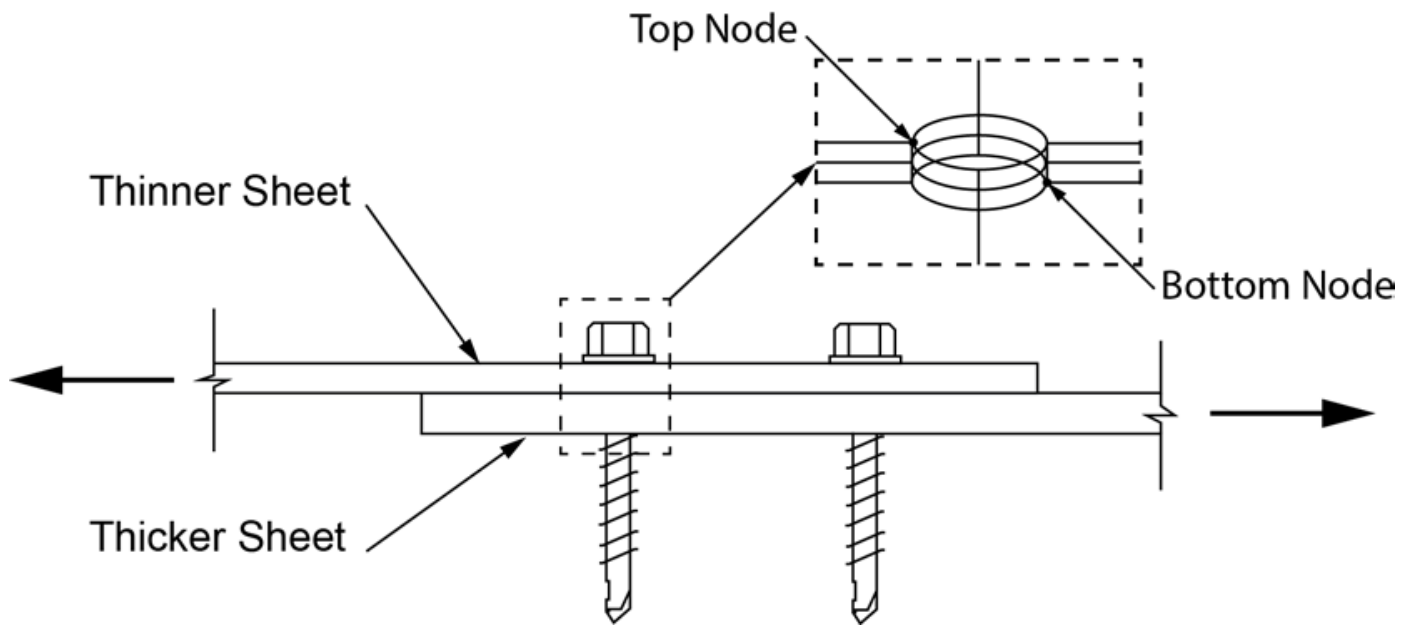

Figure 4-2 Reference nodes at screw holes for estimation of displacement of connection

\subsubsection{Variation in the sheet steel thickness}

The FEA-to-predicted results for the variation in sheet steel thickness are given in Table 4-1. When considering the variation in sheet steel thickness, it can be seen that all the FEA effectively predicted the correct mode of failure for all the design standards presented in Section 2.2. The deflection bearing/tilting resistance of the screw specimens determined using the Eurocode 3 Design Standard (2006), given in equation (2-9), provided the most conservative estimate of the deflection load carrying capacity. The FEA-to-predicted ratios all exceed 1.0 for all combinations with ratios ranging from 1.16 to 1.40 , with a COV of 0.057.

This conservative trend was not evident for all specimens when using the AISI (2007) and AS/NZS 4600 (2005) Design Standards, as determined through equations (2-4) to (2-8), when compared to the deflection based load at $3.0 \mathrm{~mm}$ elongation. Both these design standards provided similar results. The FEA-to-predicted ratios for the AISI (2007) Design Standard ranged from 0.70 to 1.09, with a COV of 0.159, where for the AS/NZS 4600 (2005) Design Standard ranged from 0.72 to 1.17 , with a COV of 0.172 . The deflection based predicted results for the AS/NZS 4600 (2005) Design Standard compared marginally better to the FEA results, and could be attributed to the increased accuracy for the bearing coefficient used, which is based on the ratio between the screw diameter and the thickness of the sheet under consideration, shown in Table 2-2. 
It should be noted that for a bearing type failure, a hole deformation of $6.35 \mathrm{~mm}$ is specified by the Research Council on Structural Connections (2004) and the American Institute of Steel Construction (1989; 2005). This is attributed to the fact that test have demonstrated that a hole elongation greater than $6.35 \mathrm{~mm}$ will begin to develop as the bearing stress is increased beyond the limiting bearing resistance values as determined through equations (2-4) to (2-8). This could thus provide a probable explanation why some of the predictions for the AISI (2007) and AS/NZS 4600 (2005) Design Standard provided low COV's. Similar ratios were observed in results presented by other researchers such as that of Rogers and Hancock (1997; 1999) as well as Yan (2012).

Table 4-1 FEA-to-predicted results for different sheet thicknesses

\begin{tabular}{|c|c|c|c|c|c|c|c|c|}
\hline \multirow{2}{*}{ Specimen } & \multicolumn{4}{|c|}{ Comparis on of Predicted Load } & \multicolumn{3}{c|}{ Comparis on of Failure Modes } \\
\cline { 2 - 10 } & $\begin{array}{c}\mathbf{P}_{\text {3.0-FEA }} \\
\text { (kN) }\end{array}$ & $\begin{array}{c}\mathbf{P}_{\text {3.0-FEA }} / \\
\mathbf{P}_{\text {AS/NZS }}\end{array}$ & $\begin{array}{c}\mathbf{P}_{\text {3.0-FEA }} / \\
\mathbf{P}_{\text {AISI }}\end{array}$ & $\begin{array}{c}\mathbf{P}_{\text {3.0-FEA }} / \\
\mathbf{P}_{\text {EC3 }}\end{array}$ & $\mathbf{P}_{\text {3.0-FEA }}$ & $\mathbf{P}_{\text {AS/NZS }}$ & $\mathbf{P}_{\text {AISI }}$ & P $_{\text {EC3 }}$ \\
\hline 080-120-S2-P-14 & 11.05 & 0.72 & 0.70 & 1.19 & $\mathrm{~B} / \mathrm{T}$ & $\mathrm{B} / \mathrm{T}$ & $\mathrm{B} / \mathrm{T}$ & $\mathrm{B} / \mathrm{T}$ \\
\hline 080-190-S2-P-14 & 16.01 & 1.00 & 0.93 & 1.24 & $\mathrm{~B} / \mathrm{T}$ & $\mathrm{B} / \mathrm{T}$ & $\mathrm{B} / \mathrm{T}$ & $\mathrm{B} / \mathrm{T}$ \\
\hline 080-300-S2-P-14 & 18.73 & 1.17 & 1.09 & 1.40 & $\mathrm{~B} / \mathrm{T}$ & $\mathrm{B} / \mathrm{T}$ & $\mathrm{B} / \mathrm{T}$ & $\mathrm{B} / \mathrm{T}$ \\
\hline 100-120-S2-P-14 & 13.74 & 0.86 & 0.86 & 1.25 & $\mathrm{~B} / \mathrm{T}$ & $\mathrm{B} / \mathrm{T}$ & $\mathrm{B} / \mathrm{T}$ & $\mathrm{B} / \mathrm{T}$ \\
\hline 100-190-S2-P-14 & 17.05 & 0.80 & 0.79 & 1.21 & $\mathrm{~B} / \mathrm{T}$ & $\mathrm{B} / \mathrm{T}$ & $\mathrm{B} / \mathrm{T}$ & $\mathrm{B} / \mathrm{T}$ \\
\hline $100-300-S 2-P-14$ & 21.65 & 1.02 & 1.01 & 1.29 & $\mathrm{~B} / \mathrm{T}$ & $\mathrm{B} / \mathrm{T}$ & $\mathrm{B} / \mathrm{T}$ & $\mathrm{B} / \mathrm{T}$ \\
\hline $120-120-S 2-P-14$ & 16.14 & 1.07 & 1.07 & 1.21 & $\mathrm{~B} / \mathrm{T}$ & $\mathrm{B} / \mathrm{T}$ & $\mathrm{B} / \mathrm{T}$ & $\mathrm{B} / \mathrm{T}$ \\
\hline $120-190-S 2-P-14$ & 18.49 & 0.72 & 0.72 & 1.16 & $\mathrm{~B} / \mathrm{T}$ & $\mathrm{B} / \mathrm{T}$ & $\mathrm{B} / \mathrm{T}$ & $\mathrm{B} / \mathrm{T}$ \\
\hline $120-300-S 2-P-14$ & 24.21 & 0.94 & 0.94 & 1.21 & $\mathrm{~B} / \mathrm{T}$ & $\mathrm{B} / \mathrm{T}$ & $\mathrm{B} / \mathrm{T}$ & $\mathrm{B} / \mathrm{T}$ \\
\hline
\end{tabular}

\subsubsection{Variation in the number of screws}

A comparison of the relative load carrying capacity of the connections, nominally identical except for the number of screws used, is presented in Table 4-2. It can be seen that when varying the number of screws arranged parallel to the applied load, the AISI (2007) and AS/NZS 4600 (2005) Design Standards differ in terms of the FEA predicted mode of failure. In the case where only one screw in the connection is considered, this difference could be attributed to the fact that the net section capacity is reduced through the use of equation (2-2). With the given dimensions, this equates to $32 \%$ of the net section capacity determined through equation (2-3). When this equation is not included in the calculations, the AISI (2007) and AS/NZS 4600 (2005) Design Standards both determine the failure to be due to bearing/tilting, as given in Table 4-3.

Table 4-2 FEA-to-predicted results for variation in the number of screws

\begin{tabular}{|l|c|c|c|c|c|c|c|c|}
\hline \multirow{2}{*}{ Specimen } & \multicolumn{4}{|c|}{ Comparis on of Predicted Load } & \multicolumn{3}{c|}{ Comparis on of Failure Modes } \\
\cline { 2 - 10 } & $\begin{array}{c}\mathbf{P}_{\text {3.0-FEA }} \\
(\mathbf{k N})\end{array}$ & $\begin{array}{c}\mathbf{P}_{\text {3.0-FEA }} / \\
\mathbf{P}_{\text {AS/NZS }}\end{array}$ & $\begin{array}{c}\mathbf{P}_{\text {3.0-FEA }} / \\
\mathbf{P}_{\text {AISI }}\end{array}$ & $\begin{array}{c}\mathbf{P}_{\text {3.0-FEA }} / \\
\mathbf{P}_{\text {EC3 }}\end{array}$ & $\mathbf{P}_{\text {3.0-FEA }}$ & $\mathbf{P}_{\text {AS/NZS }}$ & $\mathbf{P}_{\text {AISI }}$ & \multirow{2}{*}{ P $_{\text {EC3 }}$} \\
\hline 120-190-S1-14 & 9.37 & 0.90 & 0.90 & 1.18 & $\mathrm{~B} / \mathrm{T}$ & $\mathrm{F}$ & $\mathrm{F}$ & $\mathrm{B} / \mathrm{T}$ \\
\hline $120-190-S 2-P-14$ & 18.65 & 0.72 & 0.72 & 1.17 & $\mathrm{~B} / \mathrm{T}$ & $\mathrm{B} / \mathrm{T}$ & $\mathrm{B} / \mathrm{T}$ & $\mathrm{B} / \mathrm{T}$ \\
\hline $120-190-S 3-P-14$ & 26.21 & 0.80 & 0.80 & 1.10 & $\mathrm{~B} / \mathrm{T}$ & $\mathrm{F}$ & $\mathrm{F}$ & $\mathrm{B} / \mathrm{T}$ \\
\hline $120-190-S 4-P-14$ & 33.63 & 1.02 & 1.02 & 1.06 & $\mathrm{~B} / \mathrm{T}$ & $\mathrm{F}$ & $\mathrm{F}$ & $\mathrm{B} / \mathrm{T}$ \\
\hline $120-190-S 5-P-14$ & 33.53 & 1.02 & 1.02 & 1.02 & $\mathrm{~F}$ & $\mathrm{~F}$ & $\mathrm{~F}$ & $\mathrm{~F}$ \\
\hline
\end{tabular}


Table 4-3 FEA-to-predicted results for variation in the number of screws excluding reduced net section in tension equation

\begin{tabular}{|l|c|c|c|c|c|c|c|c|}
\hline \multirow{2}{*}{ Specimen } & \multicolumn{3}{|c|}{ Comparis on of Predicted Load } & \multicolumn{3}{c|}{ Comparis on of Failure Modes } \\
\cline { 2 - 10 } & $\begin{array}{c}\mathbf{P}_{\text {3.0-FEA }} \\
(\mathbf{k N})\end{array}$ & $\begin{array}{c}\mathbf{P}_{\text {3.0-FEA }} / \\
\mathbf{P}_{\text {AS/NZS }}\end{array}$ & $\begin{array}{c}\text { P }_{\text {3.0-FEA }} / \\
\mathbf{P}_{\text {AISI }}\end{array}$ & $\begin{array}{c}\mathbf{P}_{\text {3.o-FEA }} / \\
\mathbf{P}_{\text {EC3 }}\end{array}$ & P $_{\text {3.0-FEA }}$ & P $_{\text {AS/NZS }}$ & P $_{\text {AISI }}$ & P EC3 \\
\hline $120-190-S 1-14$ & 9.37 & 0.73 & 0.73 & 1.18 & $\mathrm{~B} / \mathrm{T}$ & $\mathrm{B} / \mathrm{T}$ & $\mathrm{B} / \mathrm{T}$ & $\mathrm{B} / \mathrm{T}$ \\
\hline $120-190-S 2-P-14$ & 18.65 & 0.72 & 0.72 & 1.17 & $\mathrm{~B} / \mathrm{T}$ & $\mathrm{B} / \mathrm{T}$ & $\mathrm{B} / \mathrm{T}$ & $\mathrm{B} / \mathrm{T}$ \\
\hline $120-190-S 3-P-14$ & 26.21 & 0.80 & 0.80 & 1.10 & $\mathrm{~B} / \mathrm{T}$ & $\mathrm{F}$ & $\mathrm{F}$ & $\mathrm{B} / \mathrm{T}$ \\
\hline $120-190-S 4-P-14$ & 33.63 & 1.02 & 1.02 & 1.06 & $\mathrm{~B} / \mathrm{T}$ & $\mathrm{F}$ & $\mathrm{F}$ & $\mathrm{B} / \mathrm{T}$ \\
\hline $120-190-S 5-P-14$ & 33.53 & 1.02 & 1.02 & 1.02 & $\mathrm{~F}$ & $\mathrm{~F}$ & $\mathrm{~F}$ & $\mathrm{~F}$ \\
\hline
\end{tabular}

It should be noted that since the deflection based load was compared to that of the equivalent design standards, the failure mode for specimen 120-190-S4-P-14 at 3 mm extension was the combined bearing/tilting failure, shown in Figure 4-3. However, the mode of failure observed after the $3 \mathrm{~mm}$ extension was in fact net section failure, shown in Figure 4-4, confirming the ultimate failure predictions based on the AISI (2007) and AS/NZS 4600 (2005) Design Standards. Regarding specimen 120-190-S3-P-14, no claims could be made regarding the ultimate mode of failure, as the analysis showed no indication of the initiation of net section fracture, even at an extension of $5 \mathrm{~mm}$.

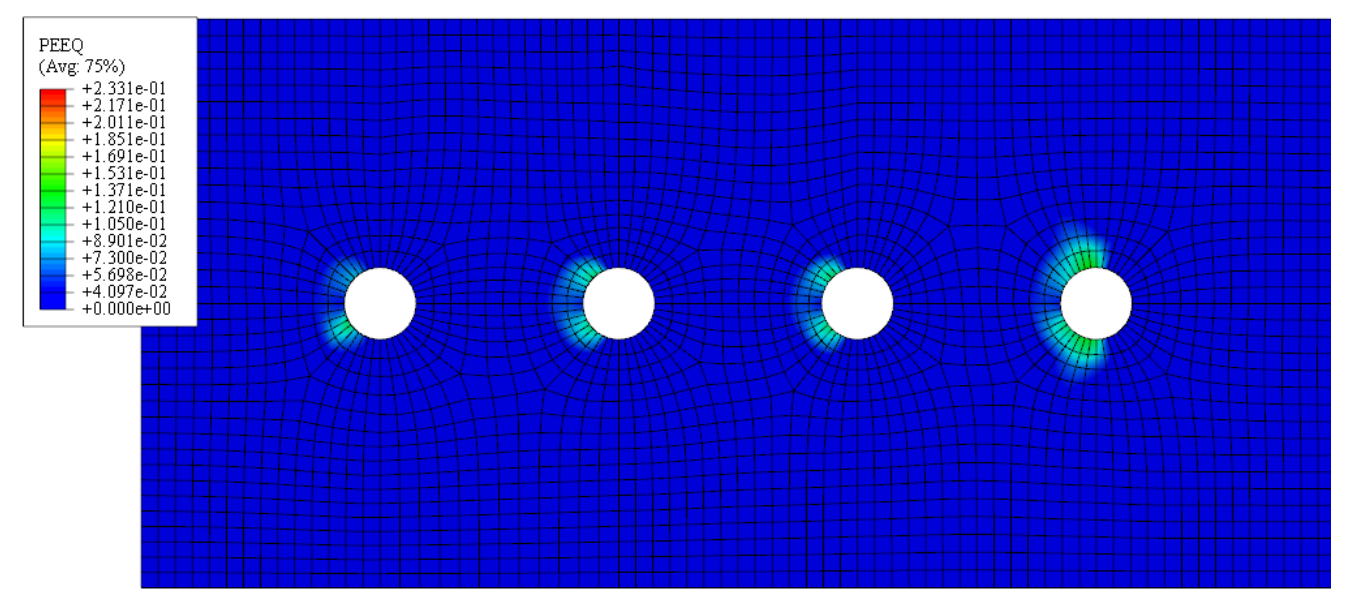

Figure 4-3 Bearing/Tilting failure for specimen 120-190-S4-P-14 at maximum deflection based load

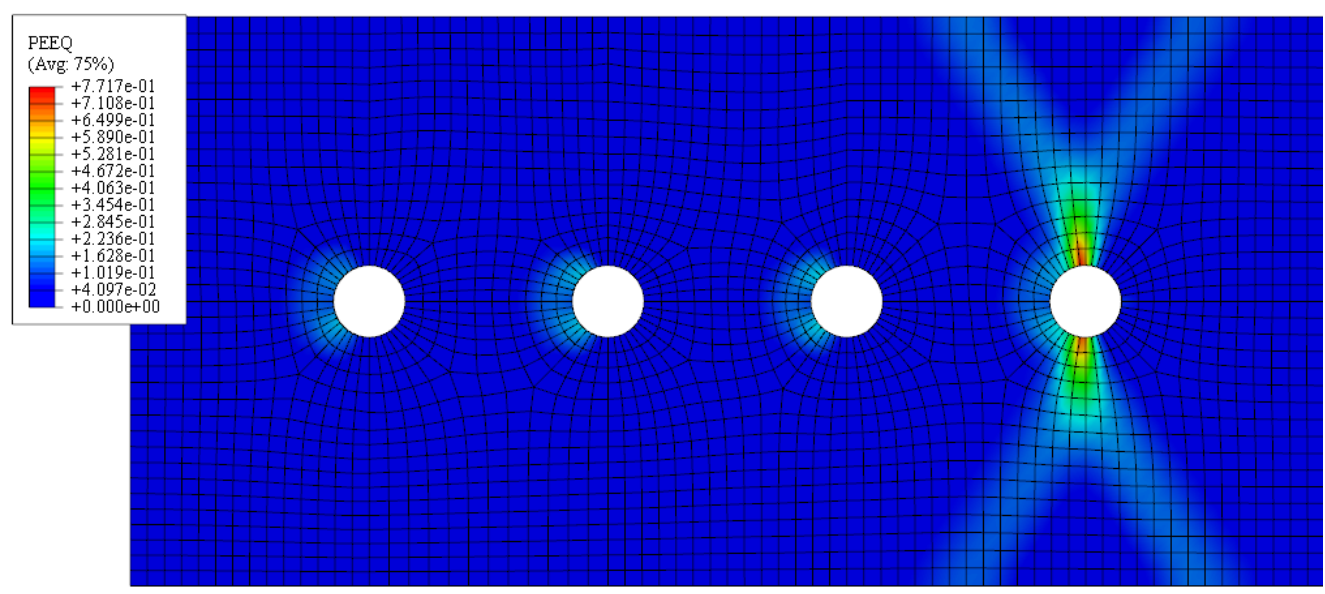

Figure 4-4 Net section fracture failure for specimen 120-190-S4-P-14 at $5 \mathrm{~mm}$ extension 
When considering the variation in the number of screws, excluding the reduced net section capacity as specified in the AISI (2007) and AS/NZS 4600 (2005) Design Standards, the FEA deflection resistance of the screwed specimens determined using the Eurocode 3 Design Standard (2006) provided the most conservative estimate of the deflection load carrying capacity. The FEA-to-predicted results all exceed 1.0 for all combinations, with ratios ranging from 1.02 to 1.18, with a COV of 0.062. This conservative trend was not evident for all specimens when using the AISI (2007) and AS/NZS 4600 (2005) Design Standards for this deflection based capacity, both providing identical results, as the FEA-to-predicted ratios ranged from 0.72 to 1.02 , with a COV of 0.178 . Similar ratios were observed in results presented by other researchers such as that of Rogers and Hancock $(1997 ; 1999)$ as well as Yan (2012).

\subsubsection{Variation in the screw size}

The FEA-to-predicted results for the variation in the size of the screws are given in Table 4-4. Once again, the FEA effectively predicted the correct mode of failure for all the design standards. The deflection bearing/tilting resistance of the screw specimens determined using the Eurocode 3 Design Standard (2006) provided the most conservative estimate of the deflection load carrying capacity. The FEA-to-predicted ratios all exceed 1.0 for all combinations, with ratios ranging from 1.16 to 1.27 , with a COV of 0.038 . This conservative trend was not evident for all specimens when using the AISI (2007) and AS/NZS 4600 (2005) Design Standards when compared to the deflection based load at $3.0 \mathrm{~mm}$ elongation. Both these design standards provided exactly the same results with FEA-to-predicted ratios ranged from 0.72 to 0.87 , with a COV of 0.085 . This could be ascribed to the bearing coefficient for both design standards being identical, even though in the AS/NZS 4600 (2005) it is based upon the ratio between the screw diameter and the thickness of the steel sheet under consideration, and in the AISI (2007) it remains constant $(C=2.7)$. From these results, it is apparent that the bearing coefficient is only affected in the AS/NZS 4600 (2005) when the thickness of the steel sheet is varied. Similar ratios were observed in results presented by other researchers such as that of Rogers and Hancock (1997; 1999) as well as Yan (2012).

Table 4-4 Comparison of FEA to predicted loads for variation in screw size

\begin{tabular}{|c|c|c|c|c|c|c|c|c|}
\hline \multirow[b]{2}{*}{ Specimen } & \multicolumn{4}{|c|}{ Comparis on of Predicted Load } & \multicolumn{4}{|c|}{ Comparis on of Failure Modes } \\
\hline & $\begin{array}{c}\text { P }_{\text {3.o-FEA }} \\
(\mathbf{k N})\end{array}$ & $\begin{array}{c}\text { P.o-feA }_{\text {3.o }} / \\
\text { P AS/SNZ }_{\text {A }}\end{array}$ & $\begin{array}{c}\text { P }_{\text {3.o-feA }} / \\
\text { PAISI }_{\text {AI }}\end{array}$ & $\begin{array}{c}\text { P }_{\text {3.o-feA }} / \\
\text { PEC } 3\end{array}$ & P3.0-FEA & $\mathbf{P}_{\text {AS/SNZ }}$ & PAISI & PEC3 \\
\hline 120-190-S2-P-8 & 14.83 & 0.87 & 0.87 & 1.27 & $\mathrm{~B} / \mathrm{T}$ & $\mathrm{B} / \mathrm{T}$ & $\mathrm{B} / \mathrm{T}$ & $\mathrm{B} / \mathrm{T}$ \\
\hline 120-190-S2-P-10 & 16.20 & 0.83 & 0.83 & 1.24 & $\mathrm{~B} / \mathrm{T}$ & $\mathrm{B} / \mathrm{T}$ & $\mathrm{B} / \mathrm{T}$ & $\mathrm{B} / \mathrm{T}$ \\
\hline 120-190-S2-P-12 & 17.24 & 0.77 & 0.77 & 1.20 & $\mathrm{~B} / \mathrm{T}$ & $\mathrm{B} / \mathrm{T}$ & $\mathrm{B} / \mathrm{T}$ & $\mathrm{B} / \mathrm{T}$ \\
\hline 120-190-S2-P-14 & 18.49 & 0.72 & 0.72 & 1.16 & $\mathrm{~B} / \mathrm{T}$ & $\mathrm{B} / \mathrm{T}$ & $\mathrm{B} / \mathrm{T}$ & $\mathrm{B} / \mathrm{T}$ \\
\hline
\end{tabular}




\subsection{EFFECT OF SHEET STEEL THICKNESS}

A total of 5 different thicknesses of thin sheet steels were used in the investigation into the effect of thin sheet steels, and included:

- Top sheet

- Bottom sheet
$1.2 \mathrm{~mm}, 1.0 \mathrm{~mm}$ and $0.8 \mathrm{~mm} \quad$ -

$1.2 \mathrm{~mm}, 1.9 \mathrm{~mm}$ and $3.0 \mathrm{~mm} \mathrm{-}$
G500 steel

G450 steel

The material properties for the various sheet steel thicknesses were assumed to be identical to that used in the verified finite element model. Hence, for the different thicknesses of the top and bottom sheet, the material properties were identical to that of the G500 $1.2 \mathrm{~mm}$ and G450 $1.9 \mathrm{~mm}$ thin sheet steel respectively. As the only parameter under consideration is the thickness of the steel sheets, the number, size and pattern of the screws were identical to that of the calibrated model, comprising two \#14 size screws arranged parallel to the applied load, at a spacing of 3d.

\subsubsection{Screw rotation}

Upon inspection of the results, it was found that where the top sheet's thickness remained constant, screw rotation decreased as the thickness of the bottom sheet increased, as depicted in Figure 4-5. This decreased rotation was also evident in simulations where the thickness of the bottom sheet remained constant, whilst the top sheet decreased in thickness, and is shown in Figure 4-6. This indicated that as the relative difference between the sheet thicknesses increased, the failure mode became more dependent upon the bearing resistance of the thinner sheet, as opposed to the combined bearing/tilting mode of failure.

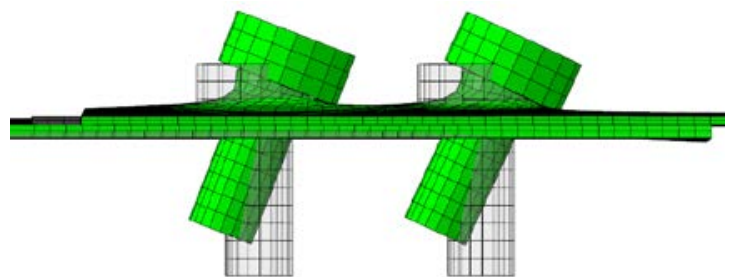

(a)

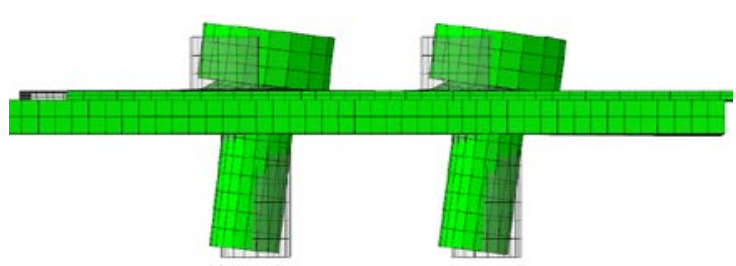

(b)

Figure 4-5 Decrease in screw rotation as bottom sheet thickness increases for specimens (a) 080120-S2-P-14; (b) 080-300-S2-P-14 when pulled to 5 mm

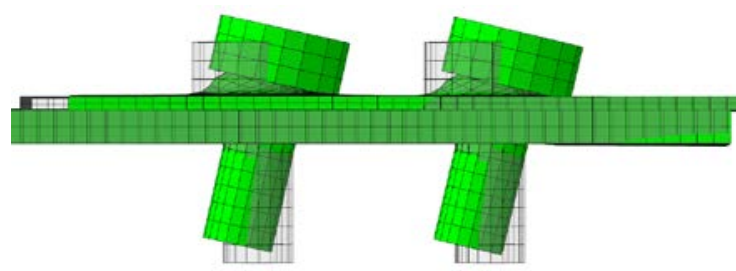

(a)

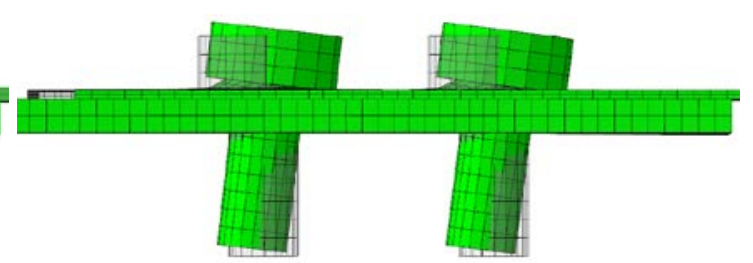

(b)

Figure 4-6 Decrease in screw rotation as top sheet thickness decreases for specimens (a) 120-300S2-P-14; (b) 080-300-S2-P-14 when pulled to $5 \mathrm{~mm}$ 
This effect was quantified through examination of the degree to which the screws rotate, as the relative difference between the thicknesses of the sheets were increased. Furthermore, the screw closest to the point where the load was applied experienced the largest degree of rotation, thus this screw was used to compare the effect of the variation in sheet thicknesses. The rotation of the screw, with respect to the ratio between the bottom $\left(t_{2}\right)$ and top thickness $\left(t_{1}\right)$, is presented in Figure 4-7. The graph shows that as the ratio between the thickness of the bottom and top sheet is increased, the rotation of the screw decreased in a linear manner.

\section{Effect of Sheet Steel Thickness on Screw Rotation}

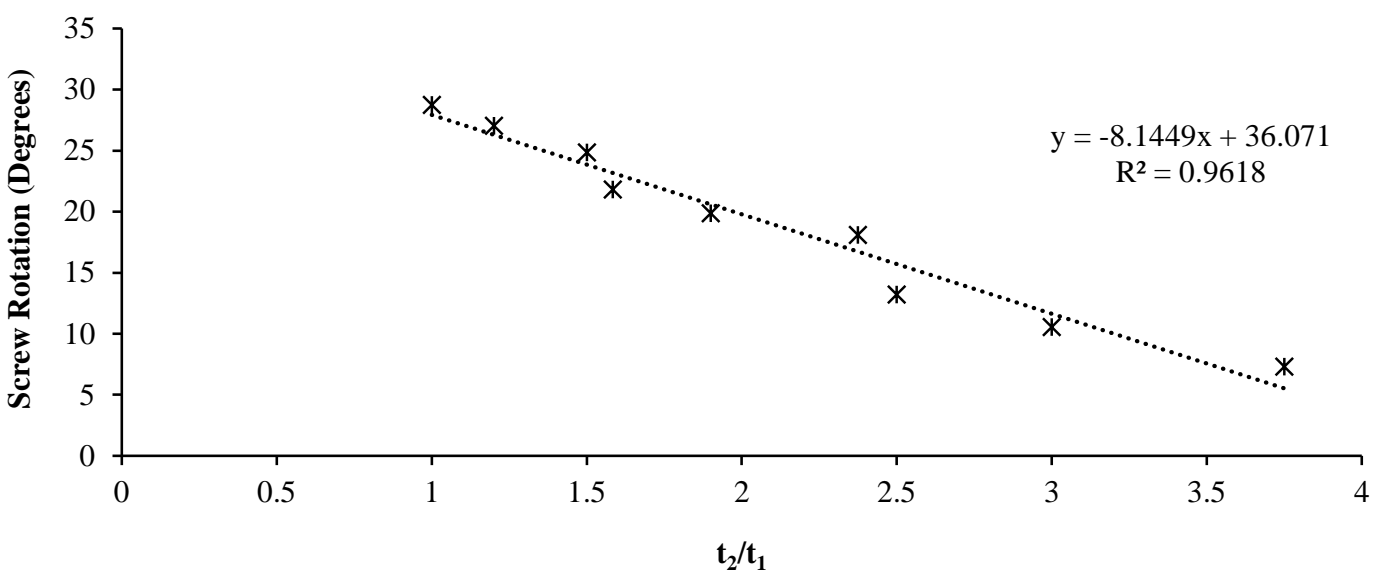

Figure 4-7 Effect of sheet steel thickness of screw rotation

\subsubsection{Stress and strain distribution}

In order to further investigate the effect of variation in sheet steel thickness, stress and strain gradient plots were extracted from the finite element analysis. The results showed that as the difference between the sheet steel thicknesses increases, the stress plots encompass similar areas across the width of the plates, however, the stress plots across the length of the plates become more columnar in shape. The von Mises stress plots exhibiting this phenomenon is shown in Figure 4-8, though this did not correlate with the results presented by Chung \& Ip (2000; 2001), who found that yield patterns were similar for sheets of the same grade but differing thicknesses. Rather, it was found that as the difference between the sheet steels increase, the yield zones are more likely to start to affect/interact with one another. 


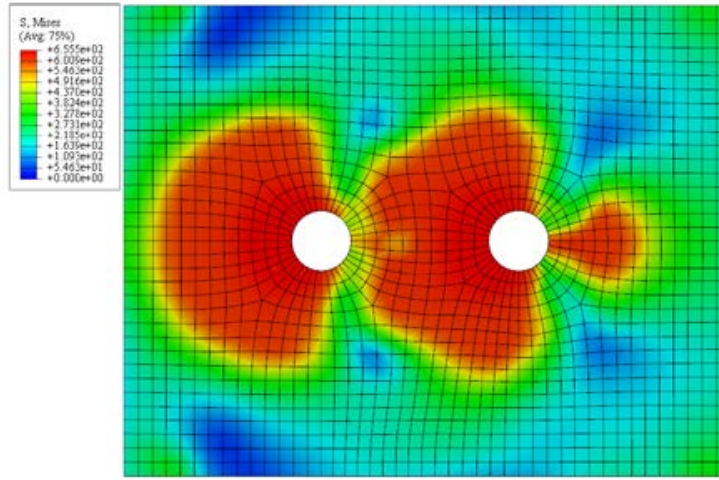

(a)
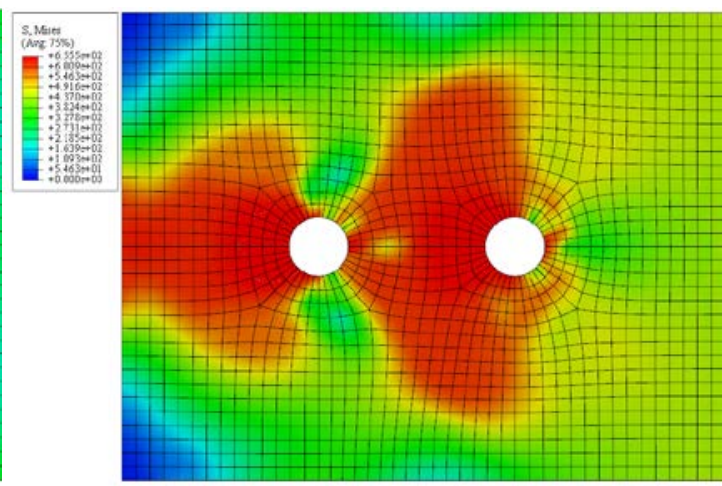

(b)

Figure 4-8 Top sheet with von Mises stress plots at $3 \mathrm{~mm}$ extension for specimens (a) 120-120-S2P-14; (b) 080-300-S2-P-14

Rogers and Hancock $(1997 ; 1999)$ postulated that for sheets of different thicknesses, the thinner element in the connection would most likely fail in bearing distress. Upon investigation of the difference in strain between the top and bottom sheets, as the difference in their thicknesses was increased, it was found that the results obtained correlated with their hypothesis. The ratio of the max principal logarithmic strain of the top $\left(\mathrm{LE}_{\mathrm{top}}\right)$ and bottom $\left(\mathrm{LE}_{\text {bot }}\right)$ sheet, with respect to the ratio between the bottom $\left(t_{2}\right)$ and top thickness $\left(t_{1}\right)$, is presented in Figure 4-9. It is shown that as the difference in thickness between the two sheet steels increased, the strain in the top sheet increased, with a corresponding decrease in strain on the bottom sheet. This concurs with Rogers and Hancock's (1997; 1999) research, whereby as the difference in the sheet thickness increases, the bearing failure becomes more dependent on the bearing resistance of the top plate, and less on that of the bottom plate, finally resulting in connection failure in a combined bearing/tilting manner, due to bearing distress in the top plate.

\section{Effect of Sheet Steel Thickness on Plate Strains}

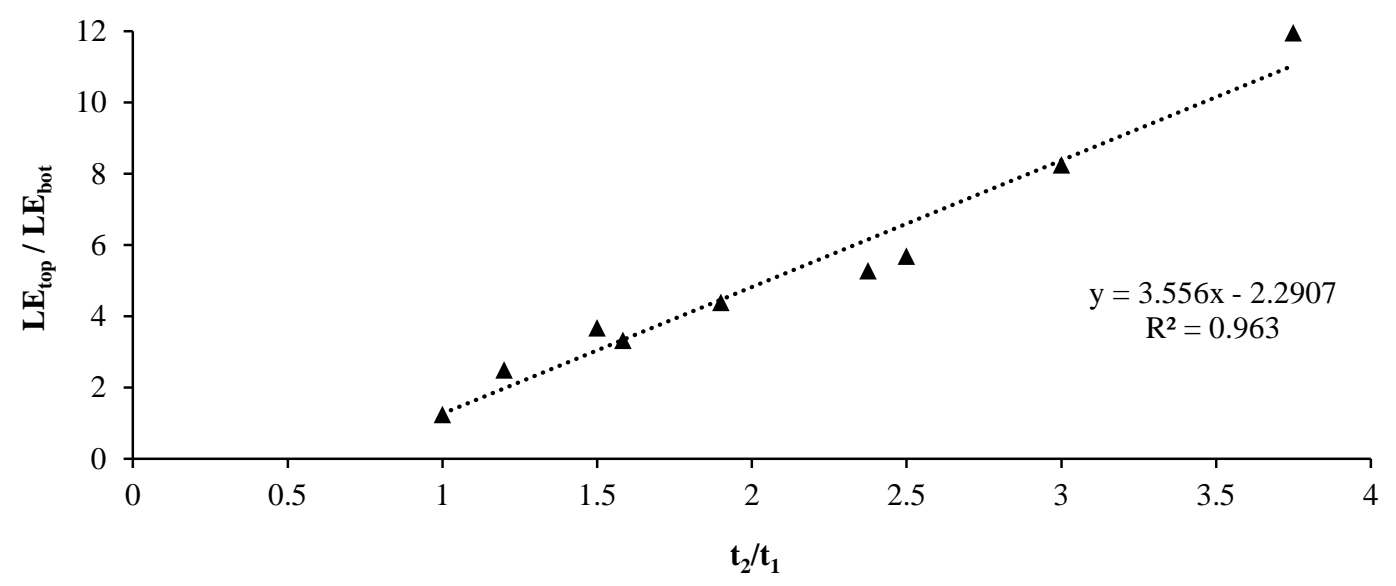

Figure 4-9 Effect of sheet steel thickness on plate strains 


\subsection{EFFECT OF NUMBER OF SCREWS}

The effect that the number of screws exerts on the behaviour of the connection was investigated through variation of the number of screws parallel to the applied load, from one to five. For the purposes of consistency, the size and spacing of the screws remained constant, thus only \#14 screws were utilised, arranged parallel to the applied load, at a spacing of $3 \mathrm{~d}$. Both the thickness and grade of the sheet steels remained identical to that of the verified model, accordingly the top and bottom sheet thickness encompassed of $1.2 \mathrm{~mm}$ and $1.9 \mathrm{~mm}$ respectively.

\subsubsection{Connection strength}

The results deduced through finite element analysis were similar to those obtained by Koka et al. (1997), whereby four or less screws resulted in tilting and bearing failure. A plot of the connection strength in terms of the number of screws in the connection is provided in Figure 4-10. A near linear relationship between the connection strength and number of screws is illustrated, until the point where the connection comprising 5 screws fails by net section fracture. Although an approximate linear increase in connection strength is depicted, this increment was not a direct multiple of the number of screws present in the connection, corresponding with the behaviour obtained from LaBoube \& Sokol (2002) and Li et al. (2010)

\section{Connection Strength vs Number of Screws}

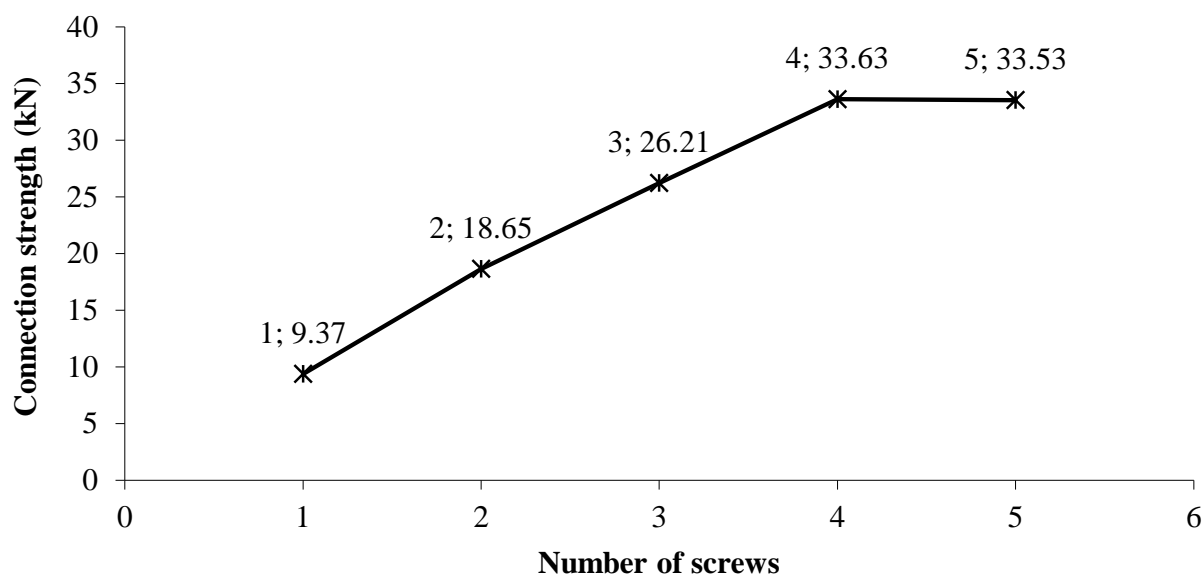

Figure 4-10 Relationship between connection strength and number of screws 
The group effect of the connections was calculated and is presented in Figure 4-11. Cognisance should be taken that 5 screws arranged parallel to the applied load resulted in the fracture of the net section, invalidating those results in terms of the behaviour ascribed to the group effect, due to the strength of the connection not being limited to its maximum bearing/tilting capacity. For connections that failed by means of tilting/bearing, comprising 4 or less screws in this instance, the group effect was not as pronounced when compared to the results obtained from LaBoube \& Sokol (2002) and Li et al. (2010). This fact could be attributed to their research comprising of steels which exhibited a higher ductility.

\section{Group Effect vs Number of Screws}

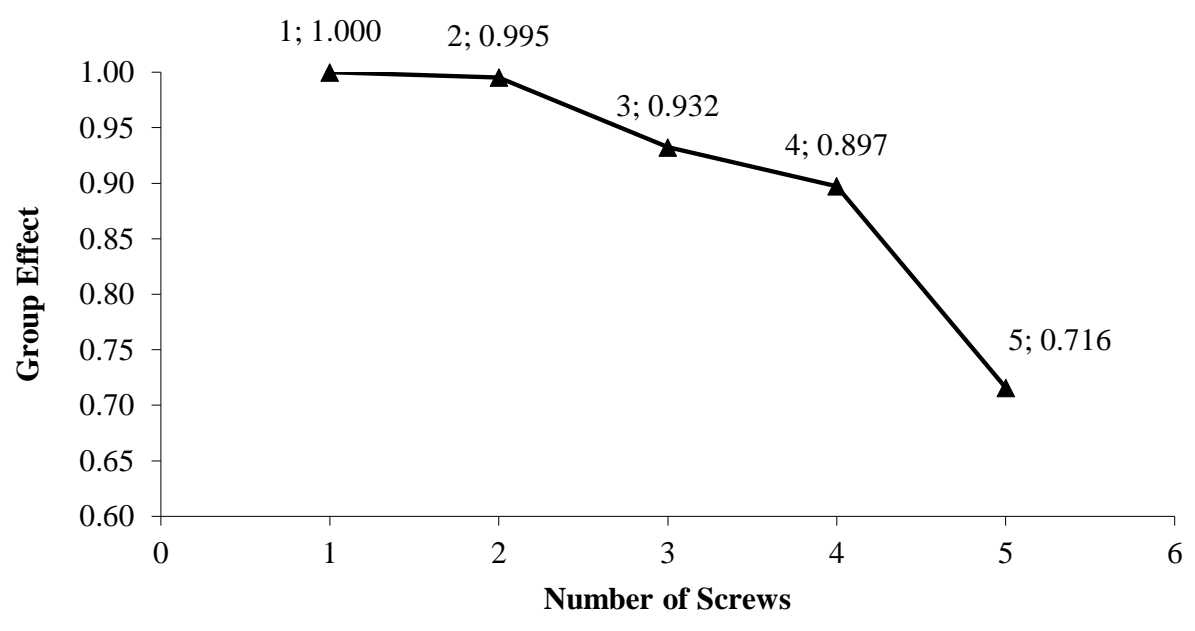

Figure 4-11 Group effect of connections against number of screws in connection

\subsubsection{Stress distribution}

Chung \& Ip (2000; 2001) noted that any reduction in the ductility of the steel exerted a significant effect on the bearing resistance of bolted connections, as a result of the yield zones being reduced during strength mobilisation. It could be posited that high-strength sheet steels are not as adversely effected by the group effect, due to reduced yield zones caused by the reduction in ductility. Shown in Figure 4-12 is the von Mises stress plot for the top plate of the three-screw connection, where it can be seen that the yield zones are not adversely affecting the behaviour of the individual screw holes. It is also shown that for the screw situated closest to the applied load on the right, the area of stress distribution is greater compared to those situated further from the applied load. 


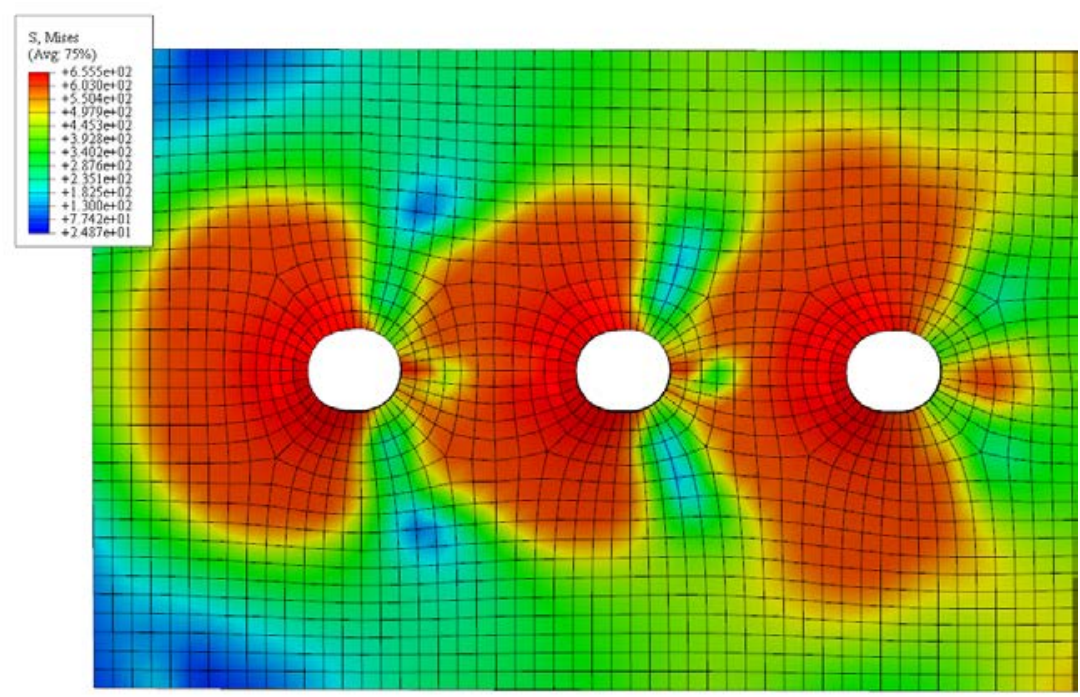

Figure 4-12 Top sheet with von Mises stress plot for specimen 120-190-S3-P-14

This phenomenon, where the screw closest to the applied load is subjected to greater stress and deformation compared to the other screws, can be visualised clearly in the five screw connection. The stress plot in the beginning phase of the deformation, where the connection still experienced behaviour typical to the bearing/tilting mode, is shown in Figure 4-13. It can be seen that the stress distribution reduces with increasing distance of the screw from the applied load. This corresponds to the fact that, provided no imperfections occur elsewhere in the sheet steel, failure of the net section through fracturing should occur at the screw located closest to the applied load, as seen in Figure 4-14.

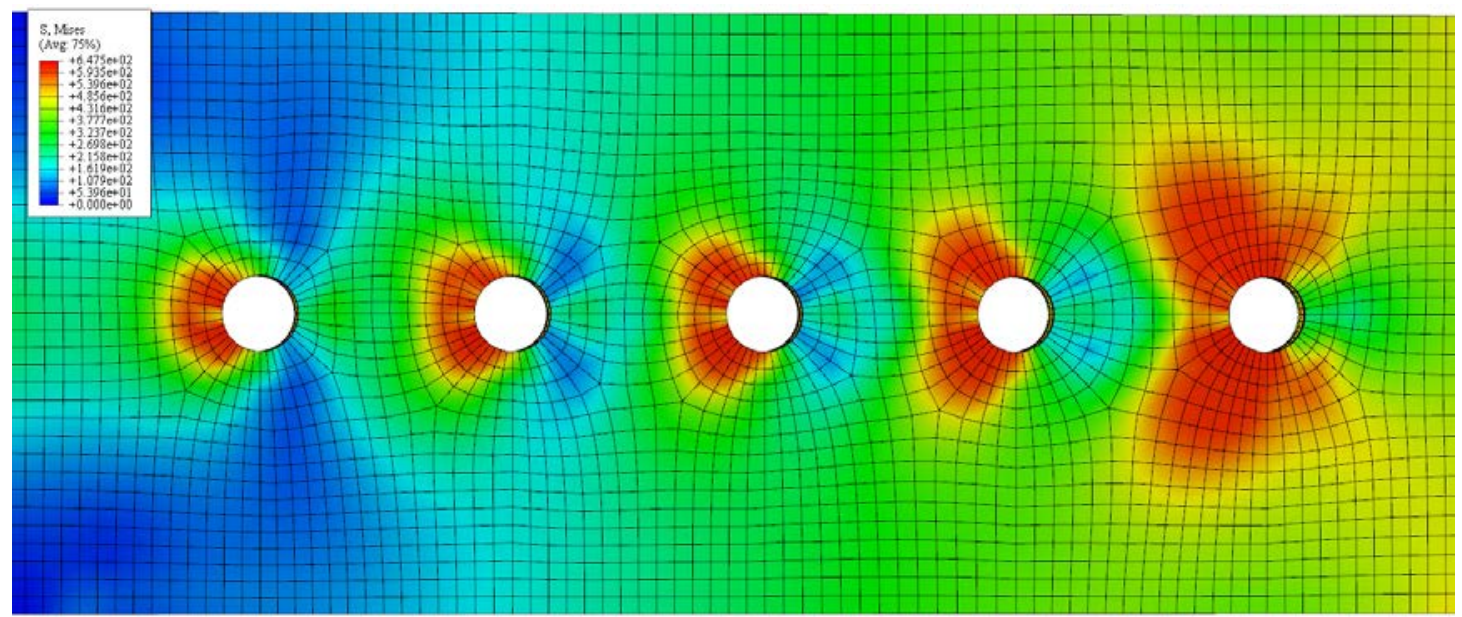

Figure 4-13 Top view of von Mises stress plot for specimen 120-190-S5-P-14 showing stress distribution variation for multiple screws 


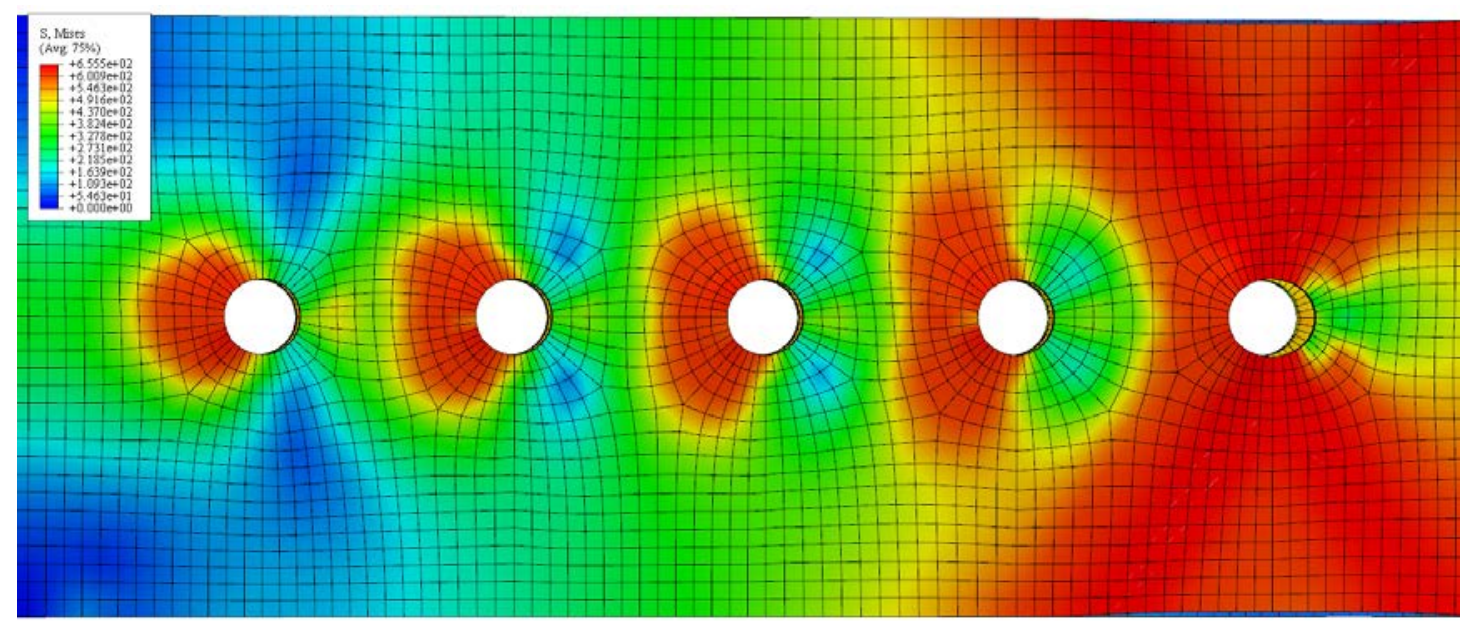

Figure 4-14 Top view of von Mises stress plot for specimen 120-190-S5-P-14 showing fracture of the net section at ultimate load

Upon inspection of the PEEQ (equivalent plastic strain) of the material, provided in Figure 4-15, it can be seen that material fracturing only occurs at the screw closest to the applied load. This "X" - shaped distribution in this specimen was also observed by Moze \& Beg (2011), which in this instance only became more pronounced after the ultimate load had been attained, as the stresses concentrated in the area where failure was occurring. Hence, it is recommended that the PEEQ should be used in order to determine the mode of failure of the specimen when using a bi-linear material curve.

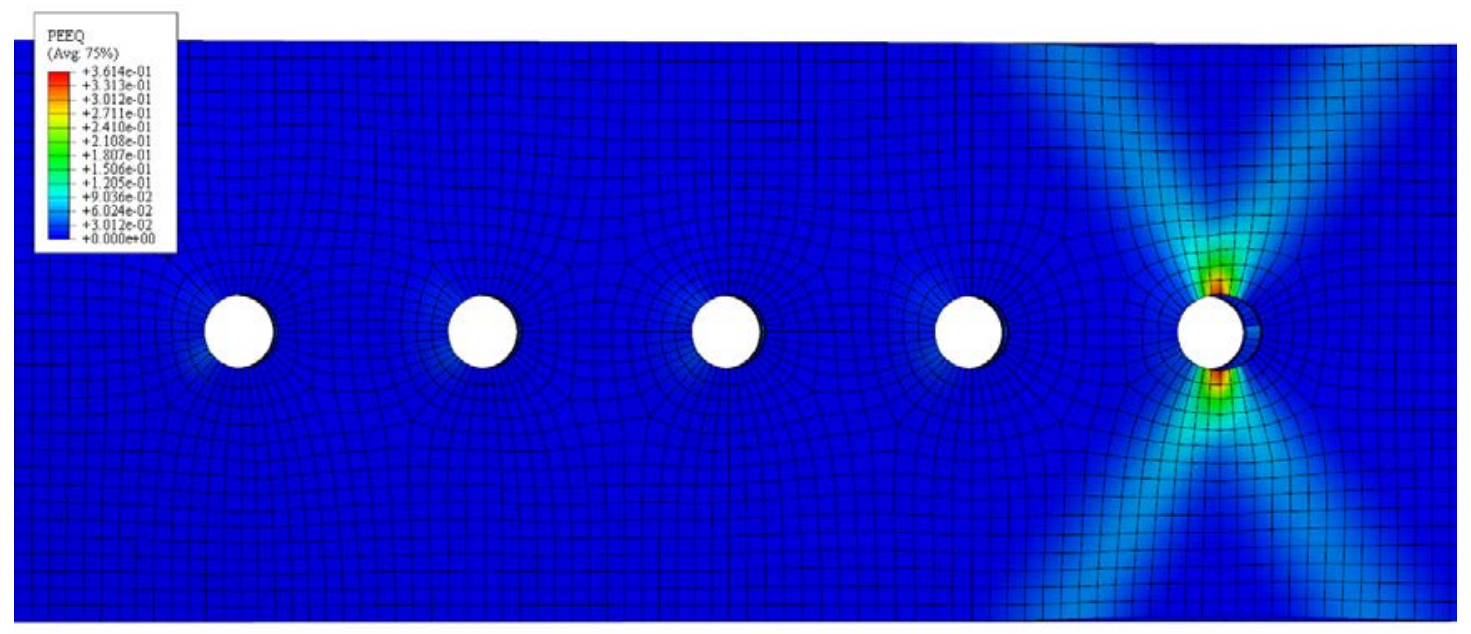

Figure 4-15 Top view with PEEQ plot for specimen 120-190-S5-P-14 showing fracture of net section at ultimate load 


\subsubsection{Screw rotation}

The effect of the number of screws, arranged perpendicular to the applied load, on the rotation of the screws was investigated. The FEA results obtained showed that the screw closest to the applied load invariably experienced the highest degree of rotation, with subsequent screws subject to consecutively decreasing degrees of rotation, as their distance from the applied load increased. The fact that the front screw experienced the highest degree of rotation was additionally illustrated in the stress and strain distribution, discussed in the previous section, which showed the stress distribution to be greatest at the screw adjacent to the applied load, decreasing as the distance from the load increased. An example of how the screws were numbered in the connection is shown in Figure 4-16. The relationship between ratios of the degree of rotation of the front screw, to that of the subsequent screws in the connection, as the number of screws in the connection was increased from 1 to 4, is shown in Figure 4-17. It should be noted that only connections failing in the combined bearing/tilting mode was included here, hence the upper limit of 4 screws with the rotations for the four screw connections extracted whilst the specimen was still in the combined bearing/tilting behaviour.

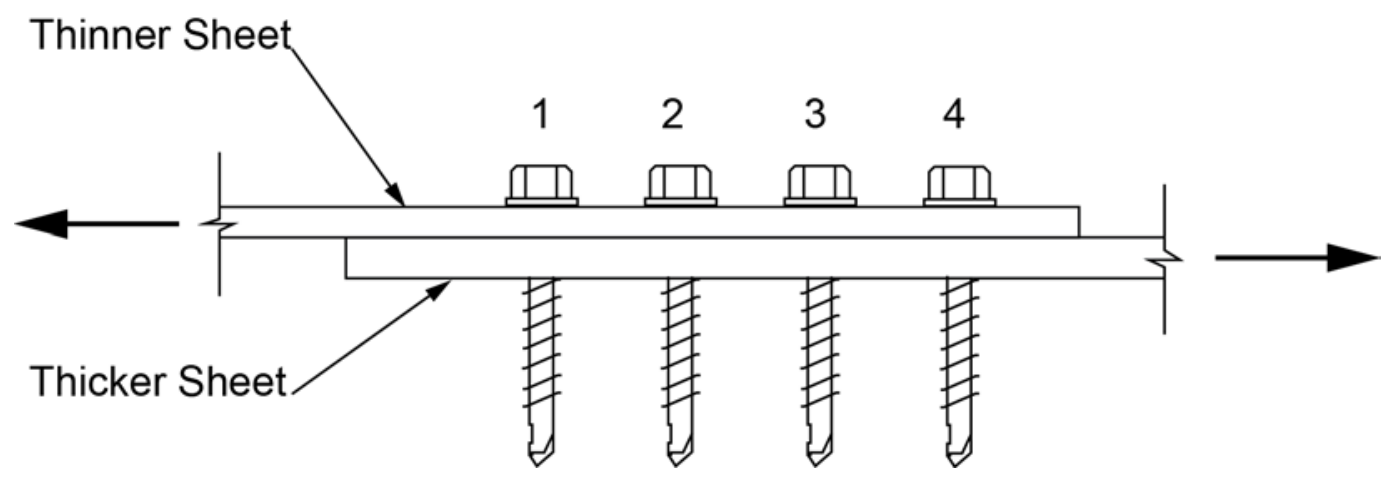

Figure 4-16 Numbering of screws arranged parallel to the applied load in connections 


\section{Effect of number of screws in connection on screw rotation}

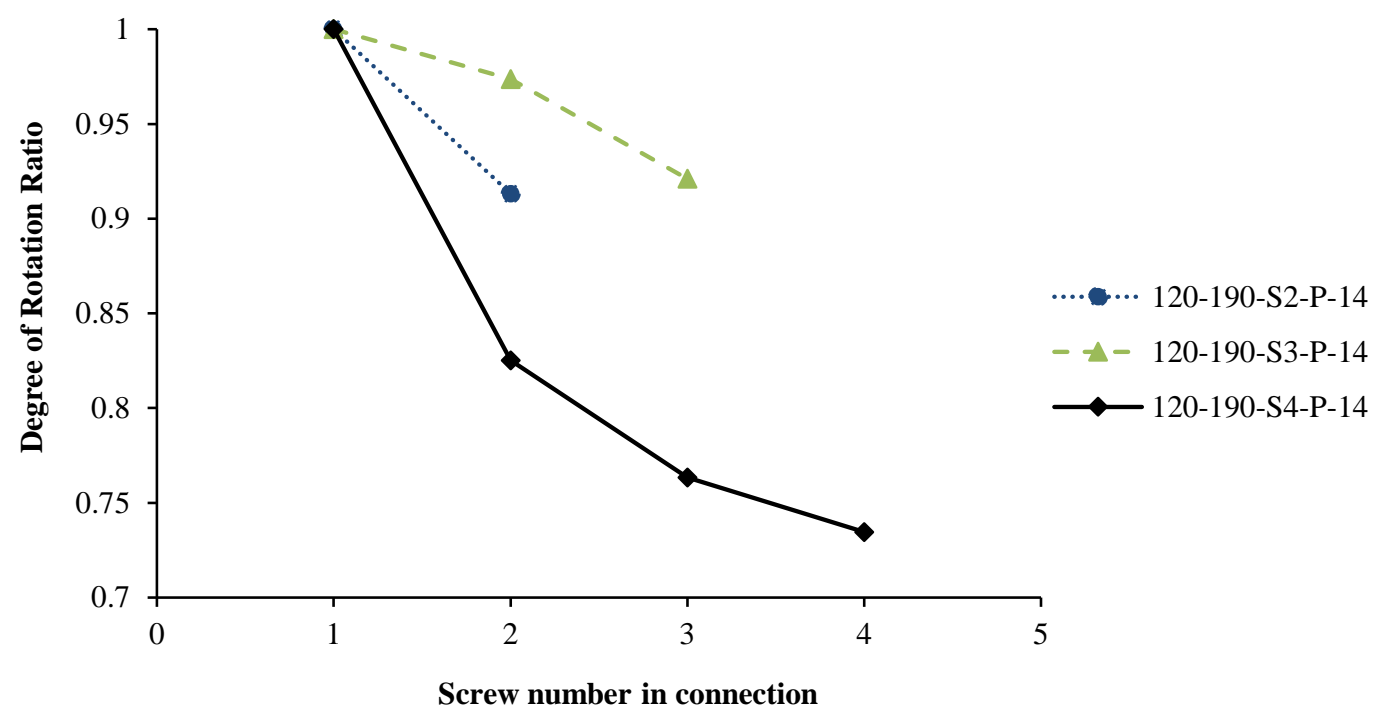

Figure 4-17 Effect of number of screws in connection on screw rotation

\subsubsection{Force-displacement curves}

The Force-displacement (F-d) curves, specifically in this instance, the Load - Hole Deformation curves, were obtained through the FEA, and are presented in Figure 4-18. Casafont et al. (2006) postulated that the shape of the F-d curve could provide an indication of the mode of failure for the connections, however, this was not as evident when examining only the F-d curves for these specimens. The most likely explanation for this would be due to the fact that a progressive damage material model was not included in the finite element model. Upon examination of the 120-190-S5-P-14 specimen, it can be seen that the load increases in a fairly linear manner, followed by a small section where tilting/bearing occurs, and once ultimate load is attained and net section fracture occurs, the graph exhibits a clear decrease in strength. It was noted, however, that this behaviour was not as obvious in the other connections. 


\section{Force-Displacement Curves for Increase in Number of Screws}

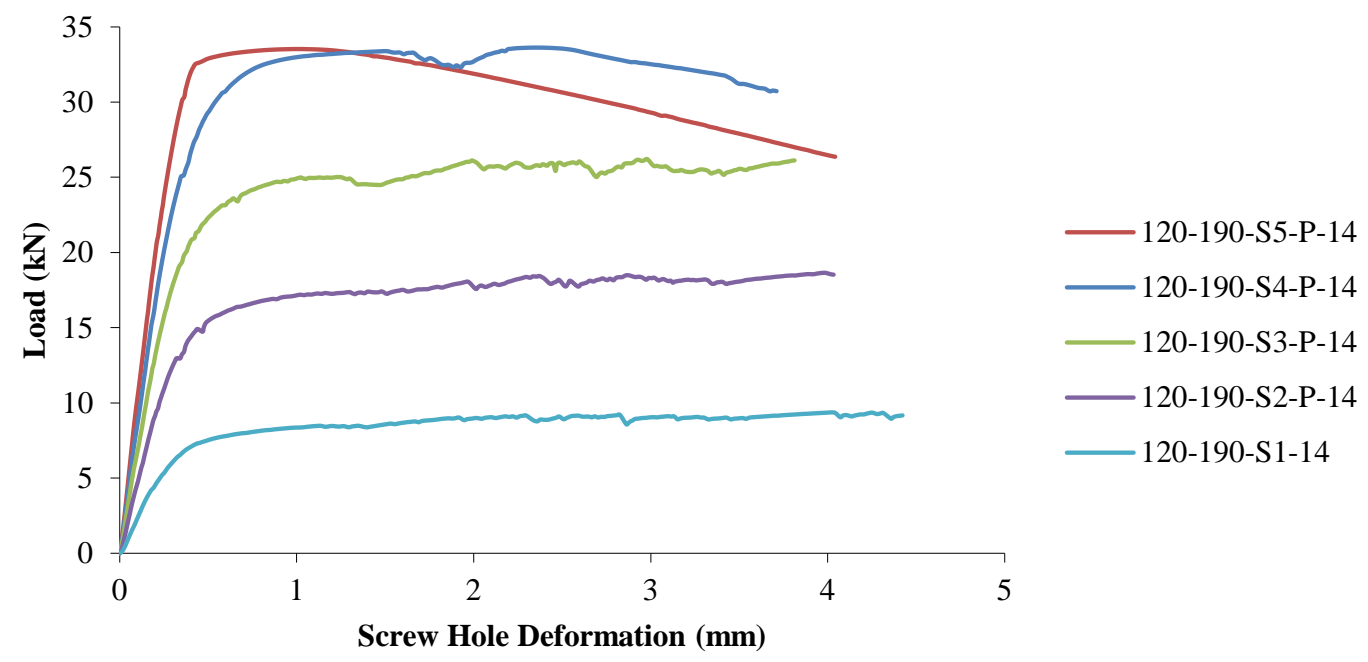

Figure 4-18 Force-displacement curves for increase in the number of screws in the connection

\subsection{EFFECT OF SCREW PATTERNS}

In the investigation into the effect of the screw patterns, four different screw patterns were considered:

- Two screws arranged parallel to the applied load (Figure 4-19a)

- Two screws arranged perpendicular to the applied load (Figure 4-19b),

- Four screws arranged in a square formation (Figure 4-19c), and

- Four screws arranged in a diamond formation (Figure 4-19d).

In order to comply with the minimum spacing and end/edge distances, as set out in Eurocode 3 (2006), and to ensure uniformity for all the geometric patterns considered, the width of the specimens was increased to $70 \mathrm{~mm}$. The size and spacing of the screws remained constant when compared to that of the verified model, necessitating the use of only the \#14 size screws, with a spacing of 3d. The thickness and grade of the sheet steels utilised also remained constant, meaning that the top and bottom sheet thickness remained $1.2 \mathrm{~mm}$ and 1.9 $\mathrm{mm}$ respectively. 
(a)

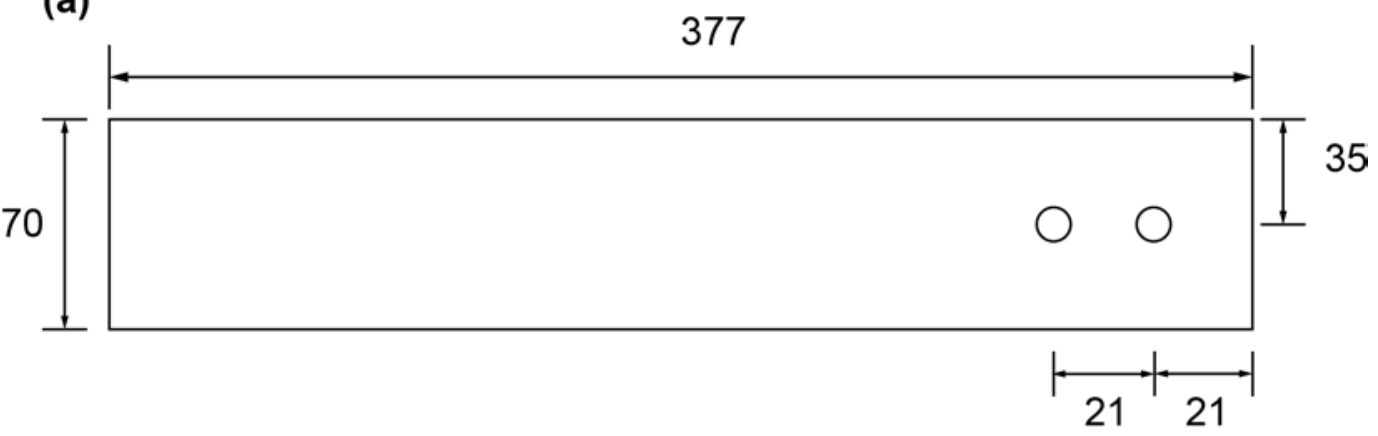

(b)

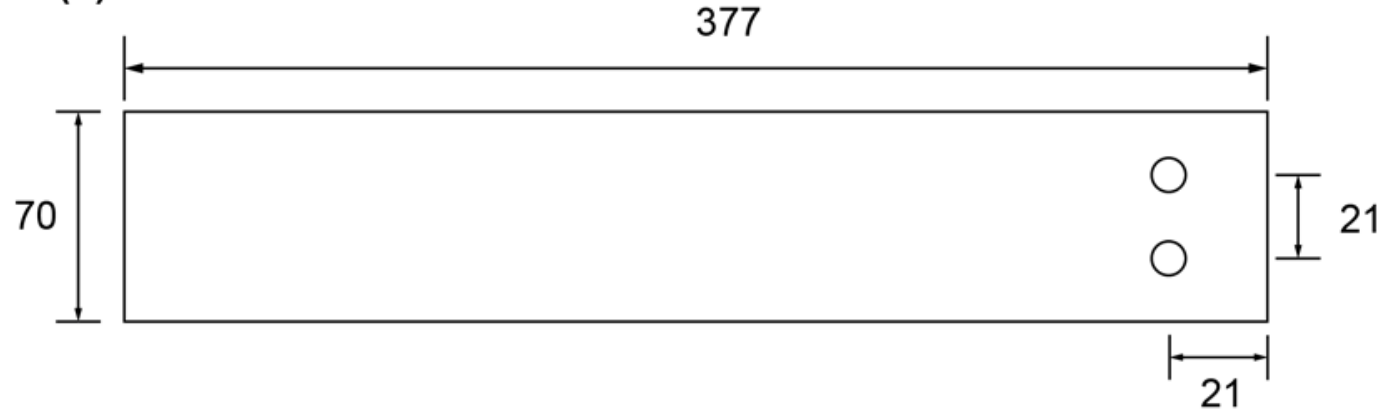

Double Screw Specimens $(\mathrm{mm})$

(c)

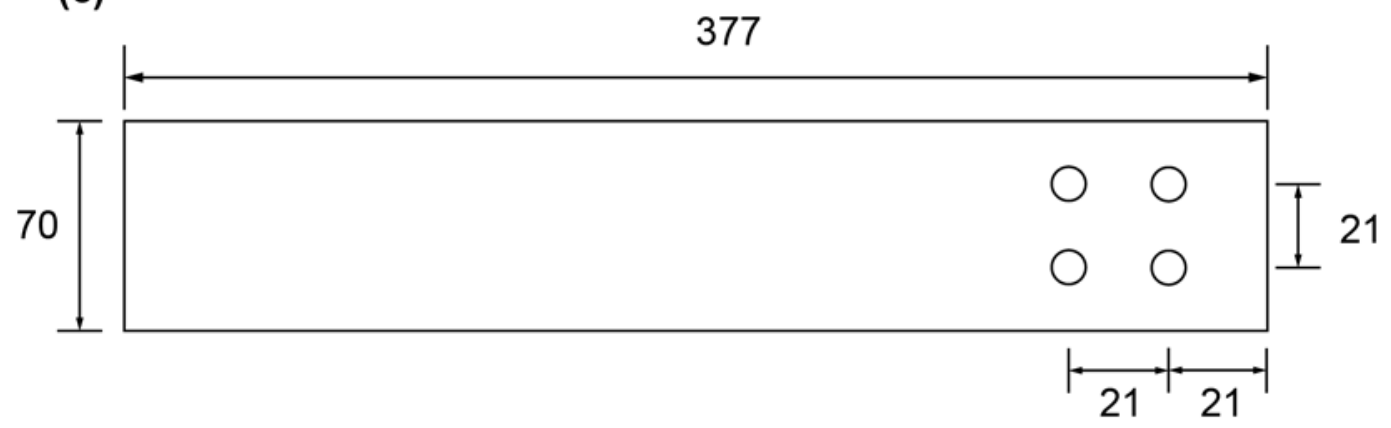

(d)

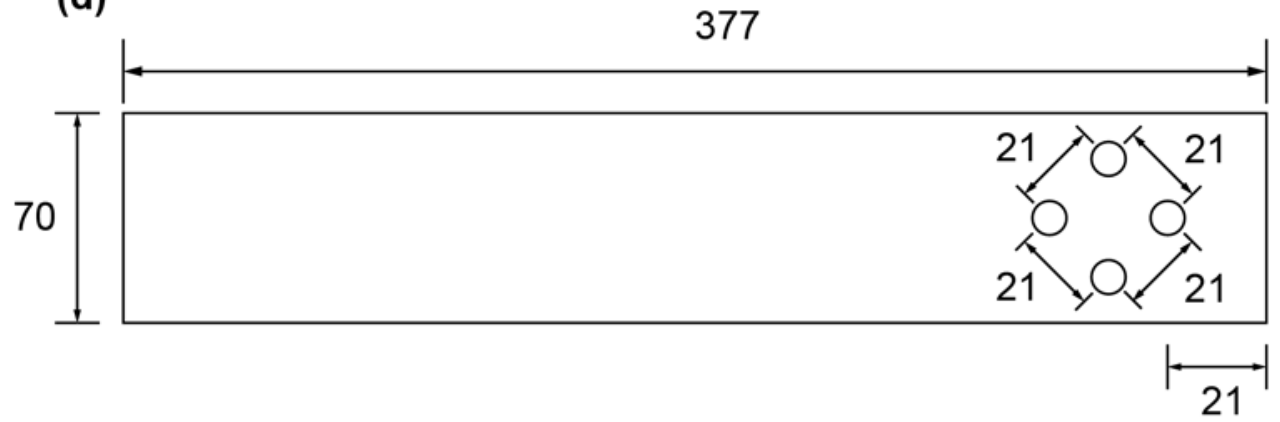

Quadruple Screw Specimens (mm)

Figure 4-19 Screw connection patterns (a) Parallel; (b) Perpendicular; (c) Square; (d) Diamond 


\subsubsection{Connection strength}

The force-displacement (F-d) graphs for the various screw patterns considered is presented in Figure 4-20. It can be observed that the different screw patterns did not significantly affect the ultimate strength of the connections. When considering the two screw connections, the specimen in which the screws were arranged parallel to the applied load (120-190-S2-P-14), performed slightly better when compared to those arranged perpendicular to the applied load (120-190-S2-V-14). When comparing the four screw connections, it is shown that the screws arranged in a square formation (120-190-S4-S-14), marginally performed better in comparison to the sample where the screws were arranged in a diamond formation (120-190S4-D-14).

\section{Force-Displacement Curves for Various Screw Patterns}

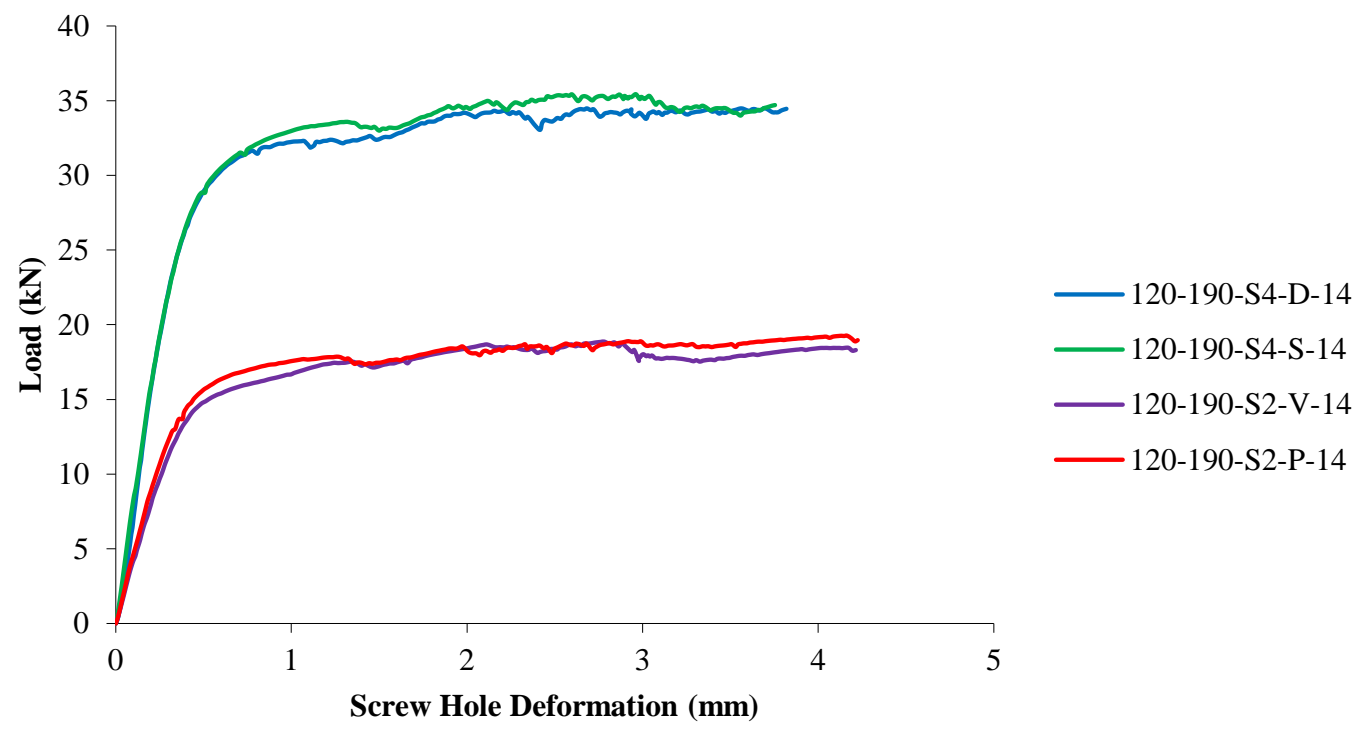

Figure 4-20 Force-displacement curves for various screw patterns

\subsubsection{Screw rotation}

Furthermore, in these specimens, an increase in the number of columns (whilst keeping the total number of screws constant) did not necessarily increase the strength of the connections, as was observed through physical tests carried out by Sokol et al. (1998). According to their research, increasing the number of columns, where the screws are arranged perpendicular to the applied load, should afford enhanced rotational stability to the connection. However, upon inspection of the degree of rotation experienced by the screws, once again this trend was not observed, as depicted in Figure 4-21 and Figure 4-22, which showed the rotation of the screws to be similar. 


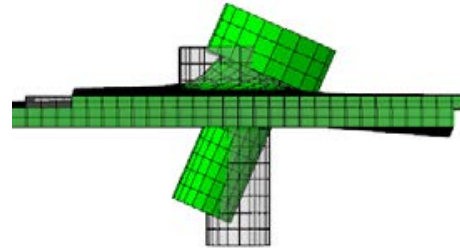

(a)

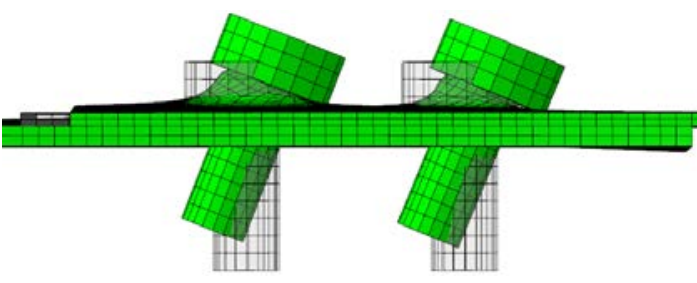

(b)

Figure 4-21 Effect of screw patterns on screw rotation for two screw connection specimens (a) 120-190-S2-V-14; (b) 120-190-S2-P-14

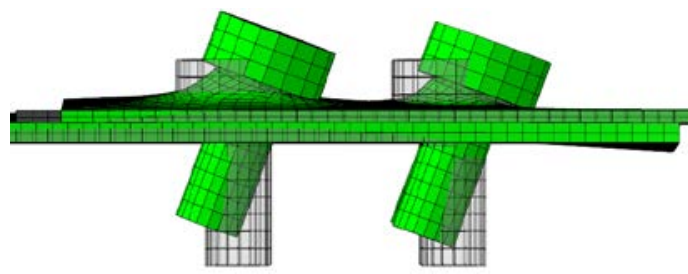

(a)

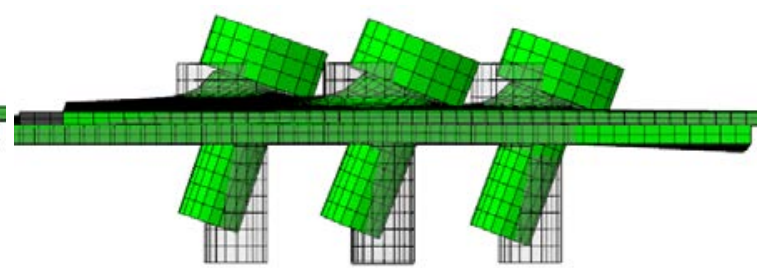

(b)

Figure 4-22 Effect of screw patterns on screw rotation for four screw connection specimens (a) 120-190-S4-S-14; (b) 120-190-S4-D-14

Quantifying the degree of rotation for each connection revealed the maximum rotation for each of the connection pairs to be similar. Once again, the front screw was proved to have the highest degree of rotation, hence these screws were used to compare the connection pairs. A comparison of the screw rotations for the connections are given in Table 4-5, where it can be observed that there are slight variations in the screw rotations, indicating that the patterning of the screws did not exert any significant influence on the connection behaviour, in terms of either screw rotation or connection strength.

Table 4-5 Effect of screw pattern on screw rotation at $5 \mathrm{~mm}$ displacement

\begin{tabular}{|c|c|}
\hline Specimen & Maximum screw rotation (Degrees) \\
\hline $120-190-S 2-P-14$ & 22.5 \\
\hline $120-190-S 2-V-14$ & 23.5 \\
\hline $120-190-S 4-S-14$ & 21.1 \\
\hline $120-190-S 4-D-14$ & 21.7 \\
\hline
\end{tabular}




\subsection{EFFECT OF SCREW SIZE}

In order to investigate the effect of the size of the screws on the connection behaviour, the screw size was varied, the smallest of which was screw gauge number \#8, up to screw size $\# 14$, the millimetre sizes of which are provided in Table 2-1. The distance between the screws were kept constant as a spacing of $21 \mathrm{~mm}$, corresponding to that of the calibrated finite element model. Additionally, the thickness and grade of the sheet steels utilised also remained the same as that of the verified model, the top and bottom sheet thicknesses remaining $1.2 \mathrm{~mm}$ and $1.9 \mathrm{~mm}$, respectively.

\subsubsection{Screw rotation}

Inspection of the FEA results showed that the screw rotation was not affected by the variation in screw size, as shown in Figure 4-23. Even though it may appear in this figure that the screw rotation is decreasing, it is only an illusion due to the smaller diameter of the screw. The rotations were quantified and the minor variations can be seen in Table 4-6.

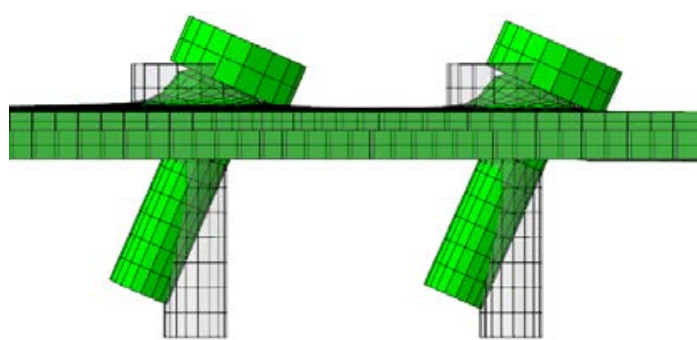

(a)

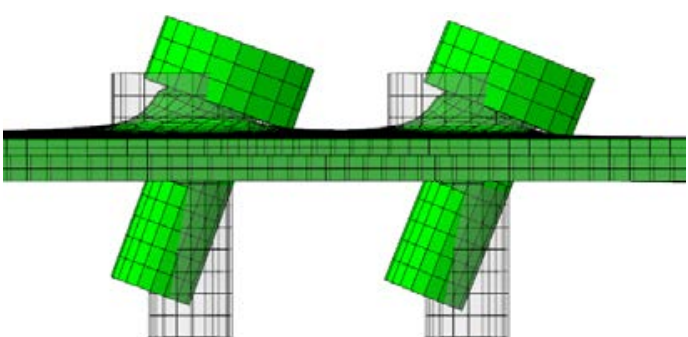

(b)

Figure 4-23 Overlay plot showing decrease in rotation with increase in screw size for specimens (a) 120-190-S2-P-8; (b) 120-190-S2-P-14 when pulled to $5 \mathrm{~mm}$ displacement

Table 4-6 Effect of screw size on screw rotation at $5 \mathrm{~mm}$ displacement

\begin{tabular}{|c|c|}
\hline Specimen & Maximum screw rotation (Degrees) \\
\hline $120-190-S 2-P-8$ & 23.8 \\
\hline $120-190-S 2-P-10$ & 21.5 \\
\hline $120-190-S 2-P-12$ & 21.7 \\
\hline $120-190-S 2-P-14$ & 20.9 \\
\hline
\end{tabular}




\subsubsection{Connection strength}

The FEA results obtained reveal that a linear relationship exists between the screw size used and the overall connection strength. A plot of the maximum connection strength, when the connection is pulled to an extension of $5 \mathrm{~mm}$, against the size of the screws is provided in Figure 4-24. The gradient of this trend was found to be quite low and thus an increase in screw size of approximately $0.66 \mathrm{~mm}$ would approximately provide a $1.2 \mathrm{kN}$ increase in connection strength (diameters corresponding to screw sizes given in Table 2-1).

\section{Effect of Screw Size on Connection Strength}

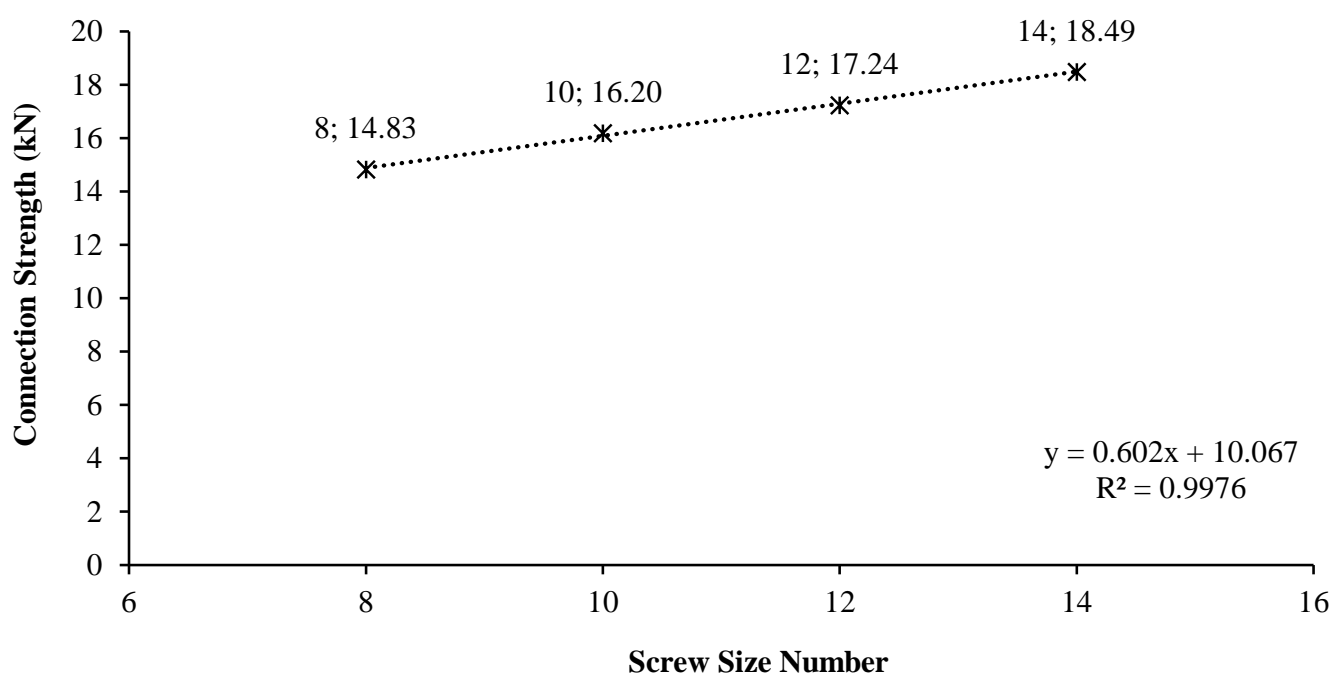

Figure 4-24 Effect of screw size on connection strength at $5 \mathbf{m m}$ displacement

\subsubsection{Stress and strain distribution}

Theories presented by Casafont et al. (2006) proposed that joints comprising of smaller diameter screws joining sheets of differing thicknesses were more prone to bearing failure. The von Mises stress plot, normalised to the diameter of the screws in the connection, is given in Figure 4-25. It can be seen that for larger screws, the intensity of the stress field is less since a larger screw provides enhanced rotational stability and also the fact that the screw itself carries more load. The stress plot for smaller diameter screw connections showed that since the screws rotate more thus provided less rotational stability, more load is placed on the steel sheets. Furthermore, the shape of the normalised stress plots are similar. These results thus agree with Casafont et al. (2006) showing that at smaller diameter screws are more prone to bearing failure since the connection behaviour is becomes more dependent on the bearing resistance of the sheet steels as the size of the screws decreases. It should be noted that FEA results revealed that the ratio between the max principal strain for the top and bottom sheet remained more or less constant, even though the stress distribution increased with an increase in screw size. 


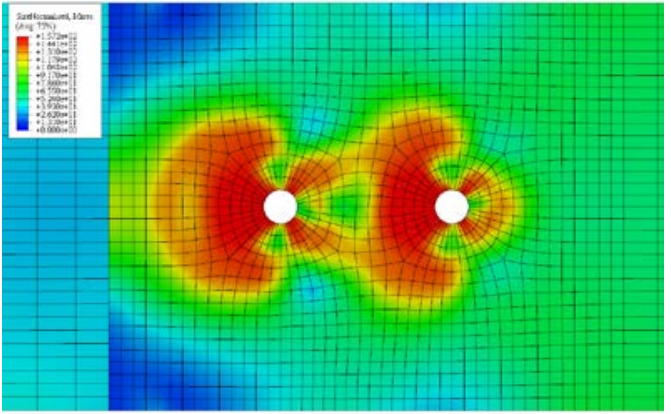

(a)

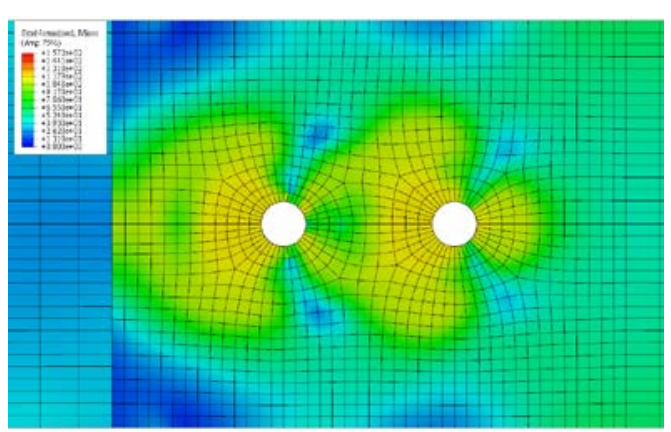

(c)

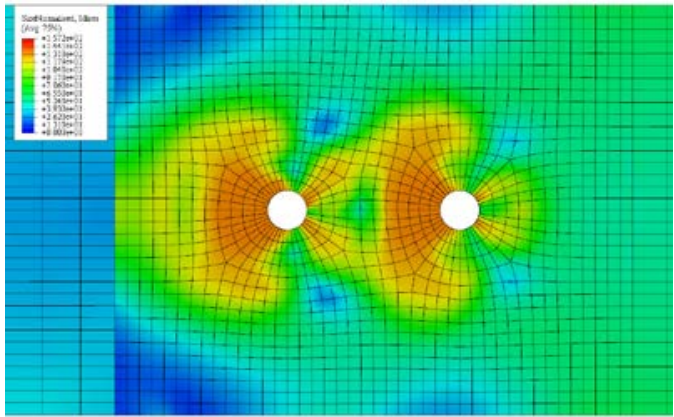

(b)

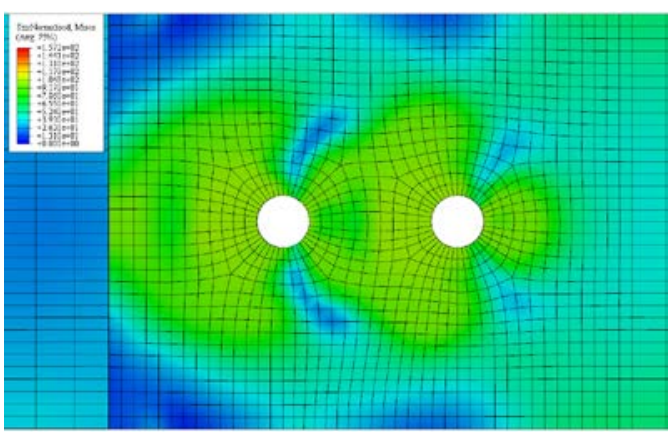

(d)

Figure 4-25 Effect of screw size on von Mises Stress distribution (normalised to size of screws in connection) for specimens (a) 120-190-S2-P-8; (b) 120-190-S2-P-10; (c) 120-190-S2-P-12; (d) 120190-S2-P-14 at $5 \mathrm{~mm}$ displacement

\subsection{SUMMARY}

The final calibrated model, as verified against the work of Yan (2012), was utilised in the subsequent parametric studies. The objective of the parametric study was to determine whether this finite element model could effectively and accurately simulate the expected experimental behaviour of screwed connections in single shear.

A total of 5 different thicknesses of thin sheet steels were examined, and included: the top sheet composed of G500 grade steel, in $0.8 \mathrm{~mm}, 1.0 \mathrm{~mm}$ and $1.2 \mathrm{~mm}$ thicknesses; and the bottom sheet composed of G450 grade steel, in $1.2 \mathrm{~mm}, 1.9 \mathrm{~mm}$ and $3.0 \mathrm{~mm}$ thicknesses. The effect of the sheet steel thickness was quantified through examination of the degree of screw rotation in the screw closest to the applied load, as the relative difference between the thicknesses of the sheets was increased. 
It was found that as the ratio between the thickness of the bottom and top sheets increased, the degree of screw rotation decreased in a linear manner. This indicated that as the relative difference between the sheet thicknesses increased, the mode of failure became more dependent upon the bearing resistance of the thinner sheet. It was postulated by Rogers and Hancock, (1997; 1999), that the thinner element in the connection would most likely fail in bearing distress, which was confirmed by the results obtained. It was also found that as the difference in the thickness of the two steel sheets increased, the strain in the thinner top sheet increased, with a corresponding decrease in strain on the thicker bottom sheet.

The effect of the number of screws used was investigated through variation of the number of screws parallel to the applied load, from one to five. The screw closest to the applied load is subjected to the greatest degree of stress and deformation, and this phenomenon can clearly be visualised in the five screw connection. Ultimately, failure of the net section through fracturing occurred at the screw located closest to the applied load, and inspection of the material PEEQ shows that material fracturing only occurs at this screw. The same " $X$ " shaped distribution found in the test specimen was also observed by Moze and Beg (2011). 


\section{$5 \quad$ CONCLUSIONS}

The objectives of the study were to investigate the finite element modelling considerations for thin sheet steel screw connections. The study was aimed at explaining the modelling technique, whereby the finite element model was calibrated with test data found in existing literature. The model had to capture both material and geometrical non-linearities. In addition, the analysis had to simulate the significant three-dimensional deformation of the thin plate connections experiencing an elongation up to $5 \mathrm{~mm}$, including the warping of the plates and the building-up of metal behind the screw head. The calibrated model was then utilised in subsequent parametric studies.

\subsection{FINITE ELEMENT MODELLING CONSIDERATIONS}

The finite element program ABAQUS 6.13 was used in the analysis for the simulation of single shear screw connections of thin sheet steels. The four main components have been carefully considered in the FEM; the steel plates, the screws, the interfaces between these two components, and the loading method. It was found that the static solver could not satisfactorily solve the large number of equations with the associated non-linearities, large deformations and contact issues. The explicit dynamic analysis was utilised in the numerical investigation, and though this method has a higher computational cost than a conventional static analysis, the FE model was simplified insofar as possible, through the use of mass scaling, and the omission of a progressive damage material model. The primary objective for this phase was the development and verification of the finite element model with the focus on the non-linear finite element modelling procedure, utilising the explicit dynamic analysis technique, and how accuracy is affected by various modelling parameters.

For the purposes of the numerical investigation, the verification process entailed the calibration of the finite element model to the work of another researcher. The decision was made to extend the study performed by Yan (2012), for the behaviour of thin sheet steel screw connections, at ambient temperatures only. The geometric dimensions of the finite element model were designed according to the details of the specimens described by Yan (2012), and the displacement controlled loading was applied up to a maximum displacement of $5 \mathrm{~mm}$ (Section 3.4.2). The finite element analysis results obtained remained within the $5 \%$ top and bottom curves obtained by Yan (2012) (Section 3.7).

\subsubsection{Effect of the contact constraint enforcement method}

Various modelling considerations were discussed whereby it was found that for the contact conditions, it is recommended that the penalty contact tracking method be employed due to the fact that even though it results in less rigorous enforcement of contact constraints, when compared to the kinematic algorithm, the penalty algorithm allows for the analysis of more general types of contact. This includes situations where there are multiple contacts per node as well as situations where a contact surface is also involved in other types of constraints (Section 3.6.1). 
In an explicit dynamic analysis, the energy output is often a significant component. In this study, the screw thread was not included in the finite element model and it was opted to rather simulate the effect of the thread artificially, through adjustment of the frictional coefficient for contact between the plate and screw shaft. It was found that due to this, the energy generated due to the frictional work will not be accurately represented. Comparison of the kinematic and penalty contact constraint enforcements' total energy output revealed that when kinematic contact enforcement is utilised, the total energy remains zero, though the model deformation was deemed to be unrealistic. It was further determined that the penalty contact constraint enforcement provided a higher level of accuracy, both in terms of the load deflection behaviour, as well as the fact that it provided a realistic deformation, although the total energy did not remain constant. This loss in total energy was attributed to the contact energy, as a result of the penalty contact constraint (Section 3.6.3).

\subsubsection{Effect of the weighting between the master and slave surfaces}

Utilising a pure master-slave contact setting, with the weight of the master surface set to 1 , decreased the loss in total energy, but also resulted in excessive penetrations into the slave surfaces. It is thus recommended that when utilising this modelling procedure to not place too much emphasis on the total energy, due to the fact that the effect of the screw thread was artificially modelled, resulting in the creation of artificial energies (Section 3.6.3). The reasons for this were ascribed to be likely due to: the model not accounting for the effect of heat generation, as a result of the increase in the friction coefficient; the lack of a fully coupled temperature-displacement analysis, as well as not accounting for the adiabatic heating effects. It was thus recommended that a balanced weighting approach be employed in the contact pair formulation (Section 3.6.2).

\subsubsection{Effective control of the applied load and how the energy output was affected}

The boundary conditions were defined so that the end of the thicker plate was fixed, and the end of the thinner plate was restrained to enable movement only in the direction of loading. Loading was then applied through the use of the displacement controlled loading method to ensure that the analysis remained in a quasi-static state. Utilising the smooth step amplitude affected the load-deflection behaviour of the model, whereby the shape of the load deflection curve mimicked the shape of the amplitude curve. Upon examination of the ratio between the kinetic energy and internal energy, the energy ratio was close to zero for the majority of the analysis, and that the application of a tabular amplitude with initial smoothing provided the best solution, ensuring that the ratio between the kinetic and internal energy not to exceed $5 \%$ (Section 3.4.3) 


\subsection{PARAMETRIC STUDY}

The final calibrated model, as verified against the work of Yan (2012), was utilised in the subsequent parametric studies to determine whether this finite element model and analysis technique could effectively and accurately simulate the expected experimental behaviour of screwed connections in single shear. The parametric study consisted of a total of 20 specimens, examining a total of 5 different thicknesses of thin sheet steels. Material properties for the top and bottom steel sheets, as well as the loading, boundary and contact conditions were kept the same as that of the calibrated model, hence all the models were only pulled to a maximum displacement of $5 \mathrm{~mm}$.

Results obtained through finite element analysis were compared to the relevant design standards (AISI S100, 2007; AS/NZS 4600, 2005; EC3, 2006). In this analysis the model was only pulled to a maximum displacement of $5 \mathrm{~mm}$, hence ultimate load for this comparison was defined to be that of a deflection based load. Reference nodes were placed, at the edges of the screw holes of the top and bottom plates. The difference between the displacements of these two nodes was used to measure the displacement of the connection. Hence, deflection based load was defined as the maximum load at/or before a screw hole deformation of $3 \mathrm{~mm}$.

\subsubsection{The efficacy of the FEM in predicting deflection limited based loads equated using the various design standards}

The results of the parametric study indicated that the current connection provisions set out in the Eurocode 3 (2006) Design Standards can be used to effectively predict the mode of failure of screwed connections at deflection based load level. In only a limited number of cases did the AISI (AISI S100, 2007) and AS/NZS (2005) Design Standards result in incorrect failure mode predictions (Section 4.2). These erroneous failure mode predictions were attributed to the fact that a comparison was made between the deflection based loads, and not ultimate loads. The inclusion of an additional check for net section failure, which is included when only one screw is in the connection, resulted in a $32 \%$ reduction in net section capacity (with these given dimensions) (Section 4.2.2).

The Eurocode 3 (2006) Design Standard provided the most conservative estimate of the deflection load carrying capacity of all screwed connections included in this study. In only a limited number of cases did the AISI (2007) and AS/NZS (2005) Design Standards provide accurate predictions for the deflection based load carrying capacity. It was also recommended that the Equivalent Plastic Strain (PEEQ) be used to determine the mode of failure of the specimens when using a bi-linear material curve, since the force-displacement (F-d) curves were found to be inadequate when determining the various mode of failures (Section 4.4.2). 


\subsubsection{Effect of sheet steel thickness in the connection}

The effect of the sheet steel thickness was quantified through examination of the degree of screw rotation in the screw closest to the applied load, as the relative difference between the thicknesses of the sheets was increased (Section 4.3.1). Variation in the sheet steel thickness showed that a linear negative relationship exists between the ratio in bottom and top sheet thicknesses and the rotation of the screws in the connection. Results further showed that as the difference between the sheet steel thicknesses increases, the strain on the top sheet increases and the strain on the bottom sheet decreases. This concurs with theories presented by Rogers and Hancock (1997; 1999) whereby as the difference in thickness increases, the bearing failure becomes more dependent on the bearing resistance of the top plate and less on that of the bottom plate, and the connection finally fails in a combined bearing/tilting failure due to bearing distress in the top plate (Section 4.3.2).

\subsubsection{Effect of the number of screws in the connection}

Results for variation in the number of screws showed that up until the point where the connection failed by net section fracture (5 screws), a linear relationship between the connection strength and number of screws existed. It was also seen that although this relationship was linear, the connection strength did not increase as a direct multiple of the number of screws present in the connection, which agrees with the results obtained from LaBoube \& Sokol (2002) and Li et al. (2010) demonstrating the existence of the group effect reduction (Section 4.4.1). It was also shown that the stress distribution for the screw situated closest to the applied load in the connection is greatest and that this stress distribution reduces as the screw is situated further and further from the applied load (Section 4.4.2). This decrease in stress distribution was mimicked by the rotation of the screws in the connections whereby the screw closest to the applied load always experienced the most rotation, with the degree of rotation decreasing the further the screw is located from the applied load (Section 4.4.3).

\subsubsection{Effect of the screw pattern in the connection}

Screw patterns were found not to have a significant effect on the connection strengths when considering two and four screw connections. It was further shown that an increase in the number of columns did not necessarily increase the strength of the connection (Section 4.5.1). The maximum screw rotations were compared and were found to be similar indicating that an increase columns did not provide enhanced rotational stability (Section 4.5.2).

\subsubsection{Effect of screw size in the connection}

Variation of the size of the screws revealed that there existed a linear positive relationship between the screw size and the connection strength. The gradient of this trend was found to be quite low and thus an increase in screw size (for example from $4.17 \mathrm{~mm}$ to $4.83 \mathrm{~mm}$ ) would approximately result in a $1.2 \mathrm{kN}$ (9 \%) increase in connection strength (Section 4.6.2). Screw rotation was found to be unaffected by the variation in the screw size (Section 4.5.2). 


\subsection{RECOMMENDATIONS}

The research on finite element modelling of thin sheet steel screwed connections is limited. As this study was based on test data found in existing literature, it is recommended that this finite element model be calibrated with experimental work, in order to certify the range of validity. Various material models, for example material models which include progressive damage models in order to accurately model the heaping of the sheet steel behind the screw or instances where net section fracture could occur, should be considered along with different types of steels, such as normal and low ductility steels should be investigated.

In this study, only one type of screw was considered, namely the self-drilling screw, hence the effect of different types of screws should be investigated to see how the finite element modelling parameters could be affected. Different screw heads, or screws combined with washers could also be investigated. An investigation into how the contact parameters are affected when very thin sheet steels are used in combination with screws with a large thread pitch should be conducted.

In this finite element analysis, specimens were only pulled until a maximum displacement of $5 \mathrm{~mm}$, hence it is recommended that specimens be pulled (and calibrated) until final failure so that the ultimate connection strength along with other deflection based loads could be compared with the predicted loads obtained from the relevant design standards. Furthermore, as this study only made use of the explicit dynamic analysis method, the general static or other analysis methods could be explored in order to determine their efficiency along with their range of applicability/validity.

The parametric study only included the change in sheet steel thicknesses and the variation in the number, size and patterns of the screws in the connections. The aforementioned parameters could of course be extended in future works, and it is recommended that the screw spacing, end and edge distances also be considered in future investigations. 


\section{REFERENCES}

ABAQUS 2015. ABAQUS analysis user's manual, Version 6.13. ABAQUS, Inc.

AISI S100 2007. North American Specification for the Design of Cold-Formed Steel Structural Members. 1002007th ed. Washington D.C., USA: American Iron and Steel Institute.

American Institute of Steel Construction 1989. Specifications for Structural Steel Buildings Allowable Stress Design and Plastic Design. Chicago: American Institue Of Steel Construction, Inc.

American Institute of Steel Construction 2005. Specifications for Structural Steel Buildings. Chicago: American Institue of Steel Construction, Inc.

ANSYS, I. 1998. Advanced Analysis Techniques Guide. 3rd ed. Canonsburg.

AS/NZS 4600 2005. Australian/New Zealand Standard - Cold-Formed Steel Structures. 46002005th ed. Sydney, Australia: Standards Australia \& Standards New Zealand.

ASTM Standard C1513-04 2007. Standard Specification for Steel Tapping Screws for ColdFormed Steel Framing Connections. ASTM International.

Barnard, J. 2011. Light-Steel Frame Building in South Africa. Walls \& Roofs 12.6(September): 30-33.

Calladine, C.R. 2011. Plasticity for Engineers: Theory and Applications. Sawston, Cambridge: Woodhead Publishing Limited.

Casafont, M., Arnedo, A., Roure, F. \& Rodriguez-Ferran, A. 2006. Experimental Testing of Joints for Seismic Design of Lightweight Structures. Part 1. Screwed Joints in Straps. Thin-Walled Structures (44): 197-210.

Chen, W.F. \& Han, D.J. 1998. Plasticity for Structural Engineers. New York: SpringerVerlag New York Inc.

Chung, K.F. \& Ip, K.H. 2000. Finite element modeling of bolted connections between coldformed steel strips and hot-rolled steels plates under static shear loading. Engineering Structures 22: 1271-84.

Chung, K.F. \& Ip, K.H. 2001. Finite element investigation on the structural behaviour of cold-formed steel bolted connections. Engineering Structures 23: 1115-25.

Daudet, L.R. \& LaBoube, R.A. 1996. Shear Behaviour Of Self Drilling Screws Used In Low Ductility Steels. In Thirteenth International Specialty Conference on Cold-Formed Steel Structures. St. Louis, Missouri, U.S.A, 1996.

Davies, J.M. 2000. Recent Research Advances in Cold-Formed Steel Structures. Journal of Constructional Steel Research 55: 267-88.

EC3 2006. Eurocode 3 - Design of Steel Structures - Part 1-3: General Rules Supplementary Rules for Cold-Formed Members and Sheeting. 1993132006th ed. Brussels: European Committe for Standardizaton. 
ECCS TC7 TWG 7.10 2009. The Testing of Connections with Mechanical Fasteners in Steel Sheeting and Sections. 2nd ed. Brussels: European Convention for Constructional Steelwork.

EN 1993-1-8 2005. Eurocode 3: Design of Steel Structures - Part 1-8: Design of Joints. Brussels: European Committee for Standardisation.

European Convention for Constructional Steelwork 1987. Recommendations for the Design of Light Gauge Steel Members.

Fan, L.X., Rondal, J. \& Cescotto, S. 1997a. Finite Element Modelling of Single Lap Screw Connections in Steel Sheeting Under Static Shear. Thin-Walled Structures 27: 165-85.

Fan, L.X., Rondal, J. \& Cescotto, S. 1997b. Numerical Simulation of Lap Screw Connections. Thin-Walled Strutures 29: 235-41.

Francka, R.M. \& LaBoube, R.A. 2010. Screw Connections Subject to Tension Pull-Out and Shear Forces. In Twentieth International Specialty Conference on Cold-Formed Steel Structures. St. Louis, Missouri, USA, 2010.

Gere, J.M. 2006. Mechanics of Materials. 6th ed. Thomson Canada Limited.

Greer, A.L., Cheng, Y.Q. \& Ma, E. 2013. Shear Bands in Metallic Glasses. Materials Science and Engineering: Reports 74(4): 71-132.

Hancock, G.J., Murray, T.M. \& Ellifritt, D.S. 2001. Cold-Formed Steel Structures to the AISI Specifications. New York: Marcel Dekker, Inc.

Kim, T.S. \& Kuwamura, H. 2007. Finite element modeling of bolted connections in thinwalled stainless steel plates under static shear. Thin-Walled Structures 45: 407-21.

Kim, T.S., Kuwamura, H. \& Cho, T.J. 2008. A parametric study on ultimate strength of single shear bolted connections with curling. Thin-Walled Structures 46: 38-53.

Koka, E.N., Yu, W. \& LaBoube, R.A. 1997. Screw and Welded Connection Behaviour Using Structural Grade 80 of A653 Steel (A Preliminary Study). Fourth Progress Report. Rolla, Missouri: University of Missouri-Rolla.

LaBoube, R.A. \& Sokol, M.A. 2002. Behaviour of Screw Connections in Residential Construction. Journal of Structural Engineering 128(January): 115- 118.

Lee, Y.H. et al. 2014. Review on Cold-Formed Steel Connections. The Scientific World Journal 2014, Article ID 951216.

Lennon, R., Pedreschi, R. \& Sinha, B.P. 1999. Comparative Study of Some Mechanical Connections in Cold Formed Steel. Construction and Building Materials 13: 109-16.

Li, Y., Ma, R. \& Yao, X. 2010. Shear Behaviour of Screw Connections in Cold-Formed ThinWalled Steel Structures. In Twentieth International Speciality Conference on ColdFormed Steel Structures. St. Louis, Missouri, 2010. 
Lu, W., Makelainen, P., Outinen, J. \& Ma, Z. 2012. Behaviour of shear connectors in coldformed steel sheeting at ambient and elevated temperatures. Thin-Walled Structures 61: 229-38.

Mac Donald, B.J. 2011. Practical Stress Analysis with Finite Elements. 2nd ed. Dublin, Ireland: Glasnevin Publishing.

Moze, P. \& Beg, D. 2011. Investigation of high strength steel connections with several bolts in double shear. Journal of constructional steel research 67: 333-47.

Pekoz, T. 1990. Design of Cold-Formed Steel Screw Connections. In Tenth International Specialty Conference on Cold-Formed Steel Structures. St. Louis, Missouri, USA, 1990.

Research Council on Structural Connections 2004. Specifications for Structural Joints Using ASTM A325 or A490 Bolts. Chicago: American Institute of Steel Construction, Inc.

Rogers, C.A. \& Hancock, G.J. 1997. Screwed Connection Tests of Thin G550 and G300 Sheet Steels. Research Report No R761. Sydney: The University of Sydney Department of Civil Engineering.

Rogers, C.A. \& Hancock, G.J. 1998. Failure Modes of Bolted Sheet Steel Connections Loaded in Shear. Research Report No R772. Sydney, Australia: The University of Sydney Department of Civil Engineering.

Rogers, C.A. \& Hancock, G.J. 1999. Screwed Connection Tests of Thin G550 and G300 Sheet Steels. Journal of Structural Engineering 125(February): 128-36.

SAE J78 2013. Steel Self-Drilling Tapping Screws. SAE International.

Salih, E.L., Gardner, L. \& Nethercot, D.A. 2010. Numerical investigation of net section failure in stainless steel bolted connections. Journal of Constructional Steel Research 66: $1455-66$.

Serrette, R. \& López, V. 1996. Performance of Self-Tapping Screws in Lap-Shear Metal-toMetal Connections. In Thirteenth International Specialty Conference on Cold-Formed Steel Structures. St.Louis, Missouri, U.S.A, 1996.

Serrette, R. \& Peyton, D. 2009. Strength of Screw Connections in Cold-Formed Steel Construction. Journal of Structural Engineering 135(August): 951-58.

Sokol, M.A., LaBoube, R.A. \& Yu, W. 1998. Determination of the Tensile and Shear Strength of Scews and The Effect of Screw Patterns on Cold-Formed Steel Connections. First Summary Report. Rolla, MO: University of Missouri-Rolla.

Tomà, A., Sedlacek, G. \& Weynand, K. 1993. Connections in Cold-Formed Steel. ThinWalled Structures 16: 219-37.

Toma, A.W. \& Stark, J.W.B. 1978. Connections in Cold-Formed Sections and Steel Sheets. In Fourth International Specialty Conference on Cold-Formed Steel Structures. St. Louis, Missouri, USA, 1978. 
Ugural, A.C. \& Fenster, S.K. 2012. Advanced Mechanics of Materials and Applied Elasticity. 5th ed. Prentice Hall.

Yan, S. 2012. Bolted and screwed connections of thin sheet steels at elevated temperatures. $\mathrm{PhD}$ report. The University of Hong Kong.

Yan, S. \& Young, B. 2011. Tests of single shear bolted connections of thin sheet steels at elevated temperatures - Part I: steady state tests. Thin-Walled Structures 49: 1320 1333.

Yan, S. \& Young, B. 2012. Screwed Connections of Thin Sheet Steels at Elevated Temperatures - Part I: Steady State Tests. Engineering Structures 35: 234-43.

Yu, W.-W. 2000. Cold-Formed Steel Design. 3rd ed. New York: John Wiley \& Sons, Inc.

Yu, H., Burgess, I.W., Davidson, J.B. \& Plank, R.J. 2008. Numerical simulation of bolted steel connections in fire using explicit dynamic analysis. Journal of Constructional Steel Research 64: 515-25. 


\section{APPENDIX A:}

\section{LIST OF DEFINITIONS}

\section{Shear Bands:}

Solids undergo plastic deformation under imposed stresses, through shearing of a section of the material, which produces a change in the material's shape. In crystalline metals, the plastic strain can often propagate throughout the material as it deforms. As the strain spreads throughout the material, the affected areas become increasingly resistant to further deformation, a phenomenon known as work-hardening. This rarely occurs in other categories of materials, with other modes of deformation, exhibiting variable degrees of shear localisation, prevailing. Shear-banding is a form of plastic instability, which localises large shear strains into a thin band as a material is deformed (Greer et al., 2013).

Shear bands are widely observed in many materials, including metals, granular solids, polymers and the earth's mantle, as shown in Figure A-1. The probability of shear banding occurring increases with high strain rates, depending on the loading conditions. The analysis of the operation of shear bands constitutes a large portion of the general theory of deformation of materials. However, shear bands are difficult to study, due to the underlying stability often being a rapid process, with the autocatalytic localisation of plastic strains, which is non-steady in time, and is spatially inhomogeneous (Greer et al., 2013).

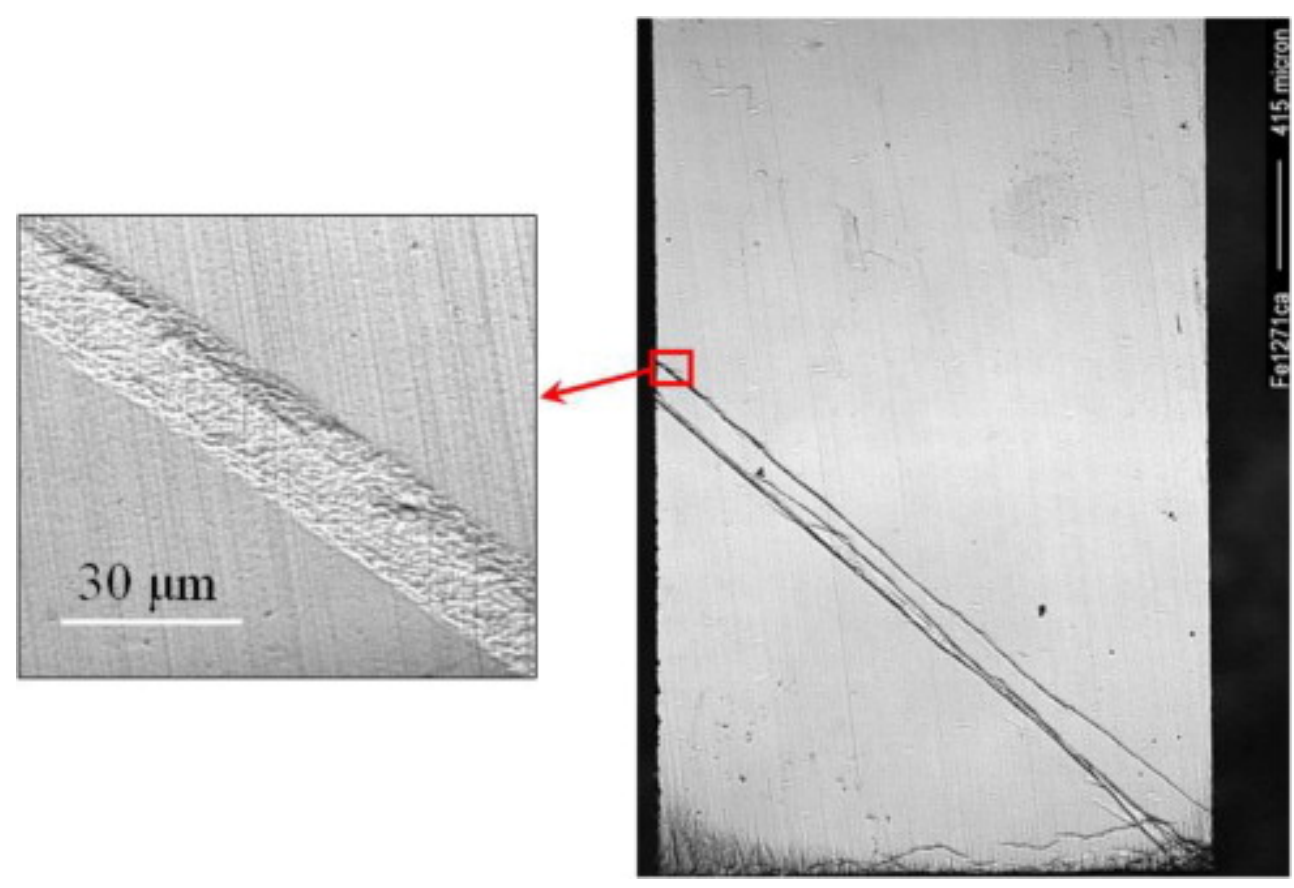

Figure A-1 Shear bands formed during compression of a consolidated cylinder of nanocrystalline Fe (grain size $80 \mathrm{~nm}$ ) (Greer et al., 2013) 


\section{Birth and Death Elements:}

The Advanced Analysis Techniques Guide for the program ANSYS (1998) describes these phenomena. To simulate the effect of "element death", instead of removing the "killed" element, the software deactivates these elements through multiplication of the stiffness of those elements with a severe reduction factor. Though the element loads associated with the deactivated elements still appear in the element-load lists, they are effectively zeroed out of the load vector. Other effects of the elements, including mass, damping, strain and specific heat, are also set to zero in these deactivated elements, and their mass and energy are not included in the model. Conversely, when elements are "born”, they are simply reactivated, and their stiffness, mass, element loads, strain, damping and specific heat return to their originally stated values, with no record of strain history.

\section{Von Mises Stress:}

Mac Donald (2011), stated that the Von Mises yield criterion is based on the assumption that, should the energy of distortion attain the same energy for yield in uniaxial tension, failure would occur. This is much less conservative compared with other yield criteria, and can be used to prevent over-designing when using ductile materials. The plane stress yield ellipse for the Von Mises failure criterion is provided in Figure A-2.

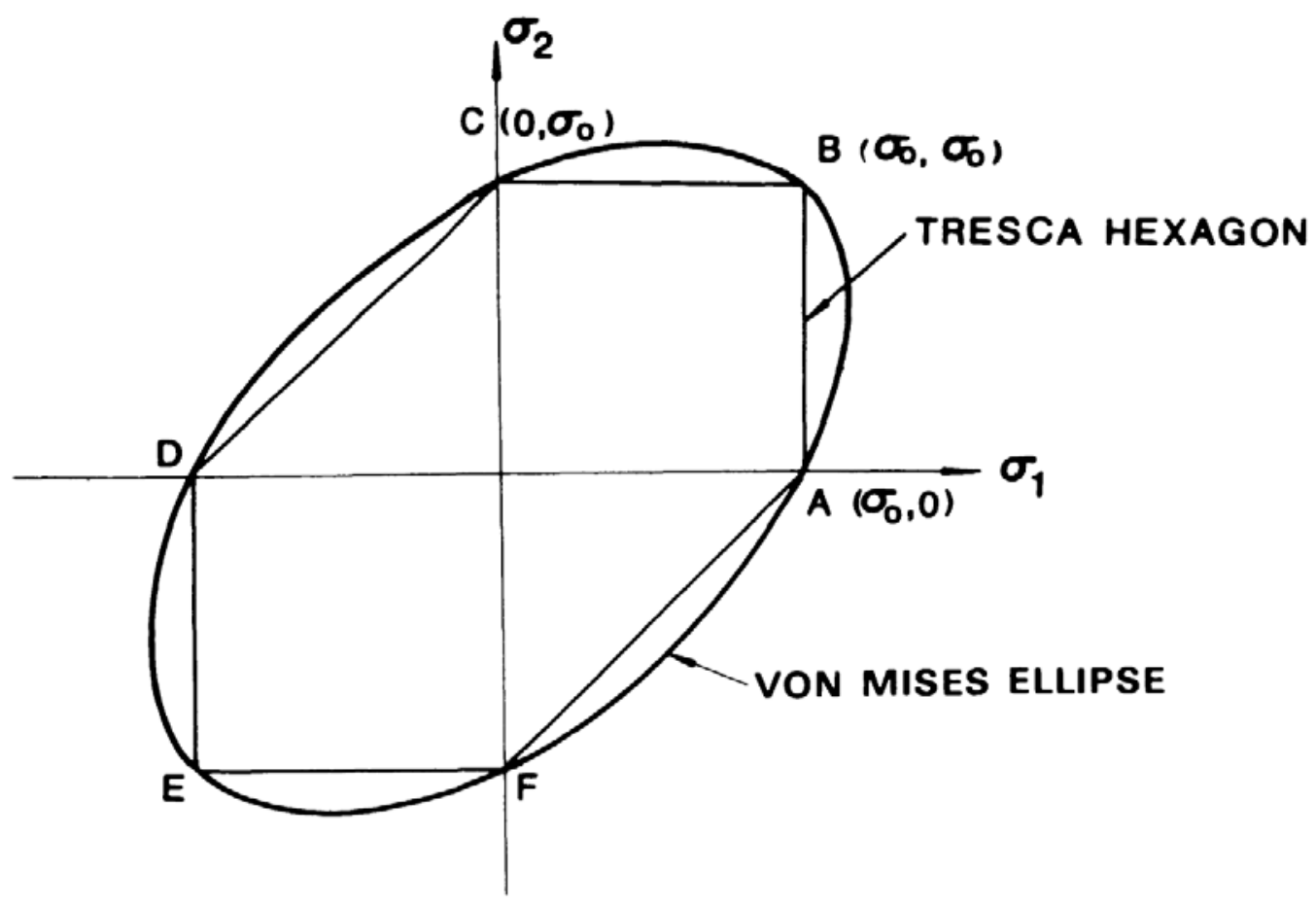

Figure A-2 Von Mises yield criteria for plane stress showing yield ellipse (Chen \& Han, 1998) 


\section{Implicit Dynamic Analysis:}

The transient dynamic analysis method, occasionally referred to as a time-history analysis, can be used to determine the dynamic response of a structure, due to the effect of any type of loading, which varies with time. This method can determine the time-varying displacements, accelerations and velocities during each time step of the analysis (Mac Donald, 2011). The equation which is solved through the employment of the transient dynamic analysis is provided here:

$$
[\boldsymbol{M}]\{\ddot{\boldsymbol{U}}\}+[\boldsymbol{C}]\{\dot{\boldsymbol{U}}\}+[\boldsymbol{K}]\{\boldsymbol{U}\}=\{\boldsymbol{f}(\boldsymbol{t})\}
$$

Where $[\mathrm{M}]$ denotes the global mass matrix; $\{\mathrm{U}\}$ the nodal displacement vector; $[\mathrm{C}]$ the global damping matrix; $[\mathrm{K}]$ the global stiffness matric and $\{\mathrm{F}(\mathrm{t})\}$ is the time-dependant load vector. During the solution, these equations also account for inertial and damping forces, and can be considered as a set of static equilibrium equations, for any time value, t. FEA solvers utilise time integration methods in order to solve these equations at discrete points, with the time increment between successive time points referred to as the "integration time step", or simply as the "time step". The majority of solvers utilise an implicit integration method, where the fundamental approach assumes that the solution for a discrete time value, $\mathrm{t}$, is known, and that the solution for a discrete time, $\mathrm{t}+\Delta \mathrm{t}$, is required where $\Delta \mathrm{t}$ is an appropriately chose time interval.

The FEA solver will increment a time step during the implicit time integration solution, and will iterate to determine if the loads or external forces applied to the structure equilibrate with the internal forces. Considering that each new time step is a function of the displacements and accelerations of the previous step, this necessitates that the equilibrium check be performed by iteration. Before the equilibrium calculations can commence, the calculation additionally requires the formation and inversion of the global model mass, damping and stiffness matrices.

\section{Explicit Dynamic Analysis:}

As previously stated, the implicit time integration method requires the formation and inversion of the global system equations at each additional step. This process is highly intensive computationally, especially when the model and the degrees of freedom become large, causing the CPU time increments to increase dramatically. This is not an issue when solving problems with few increments, but when a large number of time increments are required, it is highly likely that the computational resources available will quickly be depleted. (Mac Donald, 2011).

Thus, explicit time integration offers a surrogate method which can rapidly and efficiently solve such problems. During explicit integration, nodal accelerations are calculated directly using Newton's Second Law $(\mathrm{F}=\mathrm{ma})$, utilising a diagonal mass matrix. As the response of each node is independent, this eradicates the requirement to generate and invert large matrices, allowing the nodal displacements and velocities to be directly calculated from the time increments and accelerations that the solution has already progressed through. 
The term "explicit" is used because the calculation refers only to the values of acceleration, displacement, force and velocity at the initiation of each new time step. As the solution is deduced on an individual element basis, this enables large 3D models, comprising thousands of degrees of freedom, to be solved using much less computational power when compared to an implicit integration (Mac Donald, 2011).

The differences between the implicit and explicit methods can by fully explained through consideration of the equation below, which depicts the general form of the implicit method:

$$
U^{n+1}=f\left(\ddot{U}^{n+1}, \dot{U}^{n+1}, U^{n}, \ldots \ldots\right)
$$

Where the current nodal displacement, $U^{n+1}$, is a function of time derivative of $U^{n+1}$, which is the nodal accelerations and velocity, which are also unknown. Thus, the implicit method iterates a solution for the nodal displacements and its time derivatives, in order to solve the equations. This requires solving simultaneous equations and hence inverting the large system matrices (Mac Donald, 2011).

Conversely, the general form of the explicit method is depicted as:

$$
U^{n+1}=f\left(U^{n}, \dot{U}^{n}, \ddot{U}^{n}, U^{n-1}, \ldots \ldots\right)
$$

Where in this case, the current nodal displacement can be seen as a function of the nodal displacement, acceleration and velocity from the preceding time step. Considering that this formation is already known, this allows the use of a diagonal mass matrix, and additionally, the global equation is a system of linear algebraic equations, which can be solved for without having to resort to using simultaneous equations.

\section{Dilatational Wave:}

A dilatational wave is an elastic body, or sound, wave, and has been most thoroughly studied in reflection and refraction seismology. Particles oscillate around a fixed point, in the direction of propagation of wave energy. Within a homogenous isotropic medium, the dilatational wave velocity, $\mathrm{V}_{\mathrm{p}}$, can be calculated through the following equation:

$$
V_{p}=[\Psi / p]^{1 / 2}
$$

Where $\Psi$ is the axial modulus, and $\mathrm{p}$ is the density of the material.

\section{Master and Slave Elements:}

Contact pairs utilise the master-slave contact algorithm, where in a pure master-slave contact setting, the nodes on the slave surface are unable to penetrate the segments which comprise the master surface, as shown in Figure A-3. This algorithm places no restrictions on the master surface, allowing it to penetrate the slave surface between nodes. This contact setting can be adapted to allow the weighting between the surfaces to be changed, for example in the case of a balanced master-slave contact setting, where both surfaces will be considered equivalent, and will be unable to penetrate each other. 


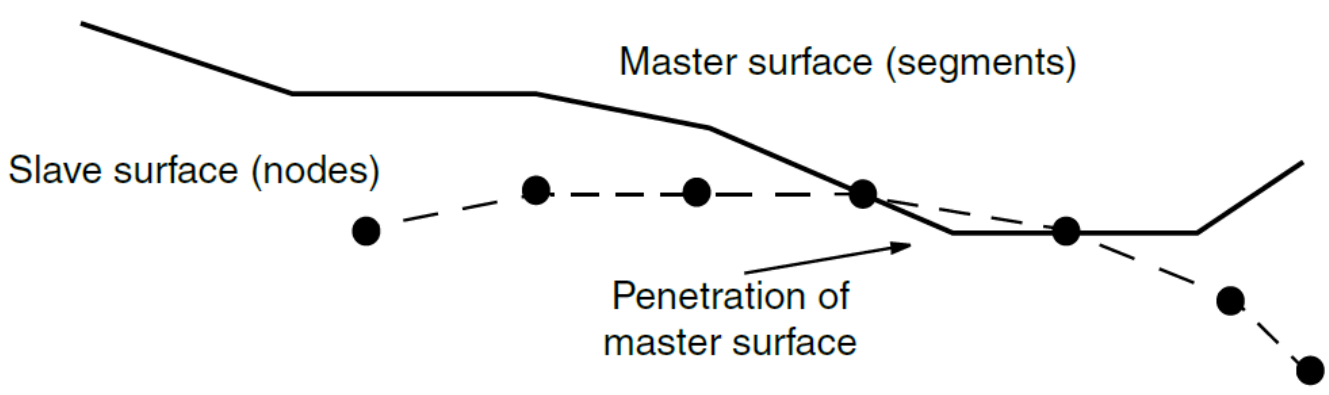

Figure A-3 Master surface allowed to penetrate slave surface (ABAQUS, 2015)

Due to the restrictions of the master-slave formulation, care must be exercised when selecting which surface is the master and which is the slave, in order to achieve the best possible simulation of contact. The following rules provide some simple guidelines regarding this selection:

- The slave surface should be more finely meshed, compared to the master surface; and

- Where the mesh densities are similar, the slave surface should comprise the softer underlying material.

\section{Shear Locking:}

In ideal circumstances, when subjected to a pure bending moment, a block of material would undergo a curved deformation, as shown in Figure A-4. As illustrated by the blue, dotted lines on the surfaces of both blocks, when subjected to a bending moment, the vertical lines will remain straight, whilst the horizontal lines will bend. It should be noted that the angle A shown on both blocks will remain at 90 degrees post-bending, as is predicted by classical beam theory (Gere, 2006).
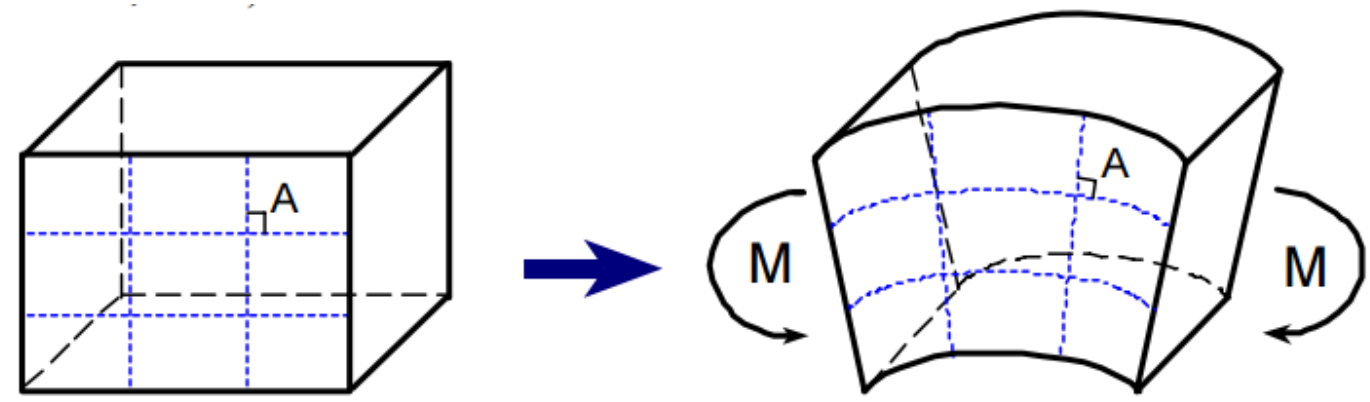

Figure A-4 Shape change of the material block under the moment in the ideal situation 
In order to properly model the ideal shape change, the element should be able to deform in such a manner as to assume the curved shape illustrated. However, fully integrated first order elements' edges are unable to deform in a curved manner, and when subjected to a pure bending moment, these linear elements will assume the shape depicted in Figure A-5. The top surface will experience a tensile stress, and congruently the bottom surface will experience an equivalent compressive stress. In such a situation, all the dotted lines will remain straight, but as is shown, angle A cannot remain at 90 degrees.
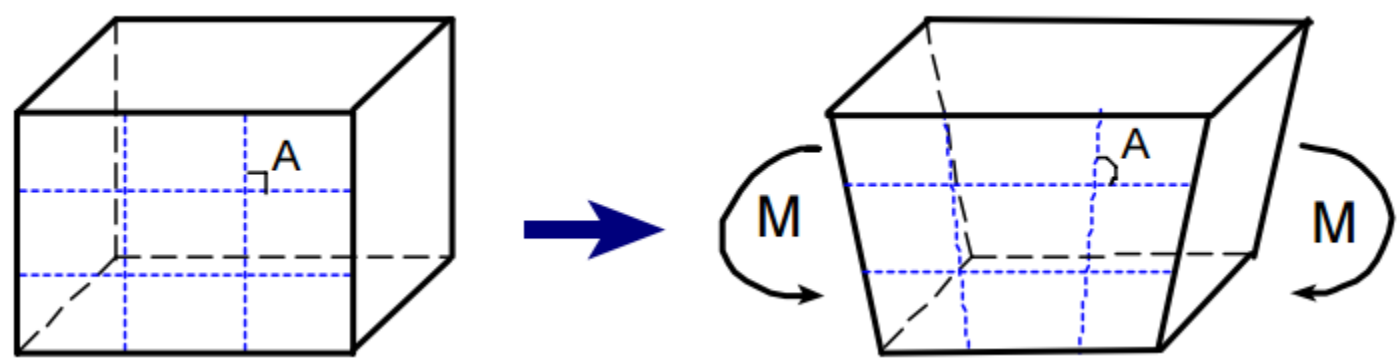

Figure A-5 Shape change of the fully integrated first order element under moment

To enable angle A to change, an incorrect artificial shear stress must be introduced. In actuality, this causes the strain energy of the element to generate a shear, instead of a bending, deformation. Essentially, the effect is such that the linear fully integrated element becomes overly stiff, or locked, under the influence of the bending moment. Due to this effect, false stresses, spurious natural frequencies or incorrect displacements may be obtained.

A fully integrated second order elements' edges are able to bend to curves, and when subjected to a bending moment, the element will assume the correct shape, as shown in Figure A-6. In this situation, angle A will remain at 90 degrees, no artificial shear stresses are introduced, and no shear locking can occur, resulting in a more realistic simulation of an actual material block.
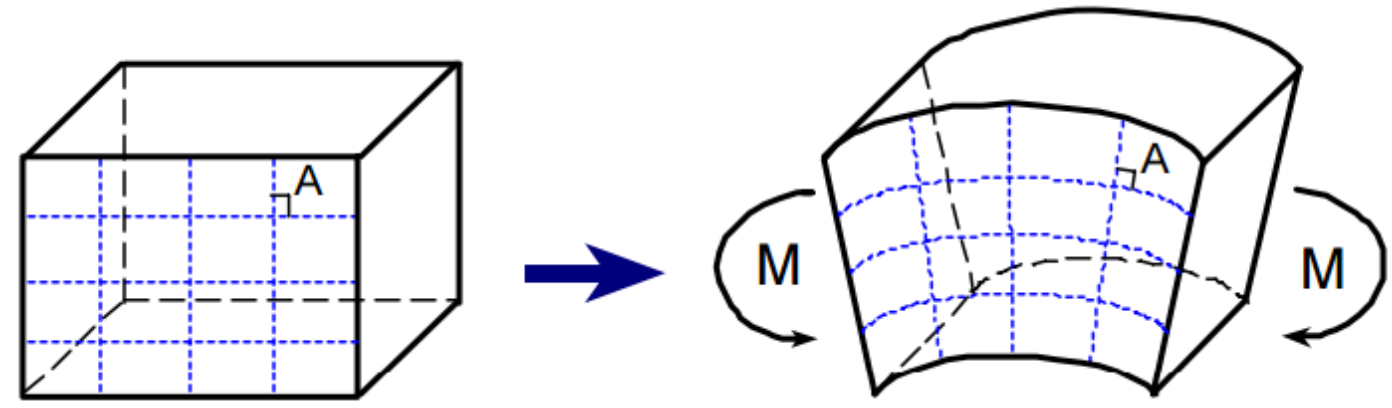

Figure A-6 Shape change of the fully integrated second order element under moment 


\section{Hourglassing:}

In order to address the problems caused by shear locking, and to increase the computational efficiency of any simulation, a reduced integration scheme could be enforced in the FEA codes. In a reduced-integration first order 8-node brick element, a single integration point scheme is used, whereas the fully integrated version will calculate for eight integration points. Similarly, a reduced integration, second order 20-node brick element would utilise an 8 integration point scheme, whilst the fully integrated version would require 27 integration points. Furthermore, the reduced integration element tolerates shape distortions, which greatly benefits the FEM.

It should be noted, however, that reduced integration first order elements undergo a phenomenon called hourglassing, as the element tends to be excessively flexible. This must be controlled, as it will invalidate the results obtained from the FEM. The deformation of such an element is illustrated in Figure A-7, where it can be observed that the vertical and horizontal dotted blue lines, and angle A, remain unchanged. This results in the normal and shear stresses being zero at the integration point, and no strain energy is generated during the deformation. This is a nonphysical response which can propagate through a coarse mesh when utilised, which would produce unusable results. The result of hourglassing may also indicate for the structure to be excessively flexible. Therefore, the FEA codes provide hourglassing control internally by default, in order to allow reduced integration elements to be of use.
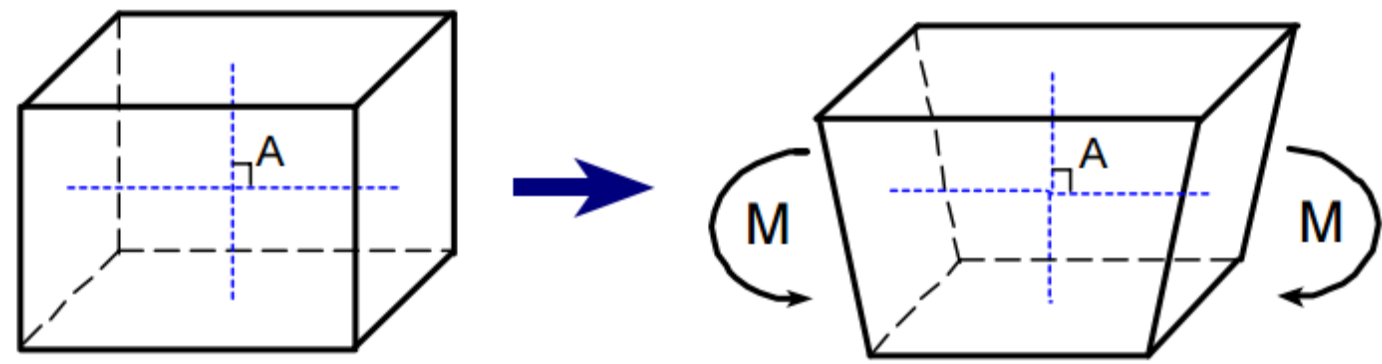

Figure A-7 Shape change of the reduced integration element under moment

Second order solid elements with reduced integration may also be subject to hourglassing, which may occur if only one layer of elements is utilised. Hourglassing essentially evanesces when two layers of elements are utilised, and requires no special technique in order to control or prevent. 


\section{Grade G Steels}

The Grade G steels mentioned in this report are created through a process known as cold reduction, which can not only produce an accurate thickness for steel sheets and related products, but can also be utilised to increase the hardness and strength of the material. The first step in the process is to roll the steel sheets to size in a hot strip mill, where the finishing and coiling temperatures are approximately $940^{\circ} \mathrm{C}$ and $670{ }^{\circ} \mathrm{C}$, respectively. The hot worked steel coil, which at this point is $2.5 \mathrm{~mm}$ thick, and has a minimum specified yield stress of 300 $\mathrm{MPa}$, is then uncoiled and subjected to an acid bath, in order to remove any surface oxides and scale which may be present. Thereafter, the uncoiled strip is trimmed to the specified size, and fed into a cold reduction mill, which may contain up to six sets of stands. The cleaning and reduction processes mentioned above can be combined, as was the case in the mill which was utilised to roll the specimens which were tested in this report. In the stands, a combination of high compressive forces and strip tension systematically reduces the thickness of the steel sheet, until the required dimensions are obtained. During this process, the thickness will be reduced by approximately 75 to $85 \%$ for the 0.60 and $0.42 \mathrm{~mm}$ steel sheets respectively (Rogers \& Hancock, 1997).

During the milling process, the grain structure of cold reduced steels is elongated along the rolling direction, which in turn produces an increase in the material strength, at the cost of decreased ductility. The effect of cold working the steel in such a manner is also cumulative, increasing the grain distortion with further cold working. It is important to note, however, that if subsequent heat treatment is used, it is possible to further change the distorted grain structure of the steel, allowing the producer to control the properties of the steel produced. There are various different types of heat treatments currently implemented, depending on the steel product which is to be augmented. G300 sheet steels are fully recrystallized, which means that the grain structure is returned entirely to its original state, though it should be mentioned that some preferred grain orientation remains. G550 sheet steels are not recrystallized, and are thus referred to as being stress relief annealed, which involves heating the steel to just below the recrystallization temperature, ensuring that the steel is heated to a constant degree throughout its thickness, followed by a slow cool-down period. Prior to the application of either a zinc or an aluminium/zinc coating, annealing is carried out in a hot dip coating. After the final cooling step, the sheet steel is further processed by means of a tension levelling mill, at $0.35 \%$ extension, in order to improve the flatness and the finish quality of the coil. The G550 sheet steels referenced in this dissertation must be differentiated from high strength, low alloy (HSLA) steels, whose high yield stress and ultimate strength are obtained through an alloying process (Rogers \& Hancock, 1997).

\section{Penalty Contact Method:}

The contact pair algorithm uses a default kinematic contact formulation, which achieves precise compliance with the contact conditions through the use of a predictor/corrector method. Initially, the increment proceeds under the assumption that contact does not occur. If there is overclosure at the end of the increment, the acceleration is modified so that a correct configuration is obtained, in which the contact constraints are enforced. 
The normal contact constraint for contact pairs can be optionally enforced using the penalty contact method, in order to model some types of contact which the kinematic model cannot. When the penalty contact formulation is utilised, equal and opposite contact forces, with magnitudes equivalent to the penalty stiffness multiplied by the penetration distance, are applied to the master and slave nodes at the penetration points.

The penalty contact algorithm results in less rigorous enforcement of contact constraints, when compared to the kinematic algorithm, though the penalty algorithm does allow for the analysis of more general types of contact. Both methods conserve momentum between the contacting bodies, and the enforcement of the contact conditions can either be by means of a pure, or a balanced, master-slave contact pair. The penalty contact method is ideally suited for very general contact modelling, including the following situations:

- Multiple contacts per node, and

- Contact of surfaces also involved in other types of constraints.

The kinematic contact algorithm stringently enforces the contact constraints whilst conserving momentum. In order to attain these qualities within a discretised model, some energy must be absorbed upon impact. To illustrate this, consider a linear elastic truss, modelled with several elements, impacting a rigid wall. Upon impact, the kinetic energy of the leading node is absorbed by the contact algorithm, whilst a stress wave propagates through the truss, eventually causing the truss to rebound from the wall. After rebounding, the kinetic energy is decreased due to the loss of the contact load's energy upon impact. As the mesh is refined, this energy loss is diminished, as a result of the mass and kinetic energy of the leading node of the truss becoming less significant.

On impact, contact forces can also exert negative external work, since they act over the entire increment in which impact occurs, including the fraction of the increment prior to the impact. The opposing contact forces, which possess equivalent magnitudes, act across different distances, thereby exerting a nonzero net work. The net external work of these forces is negative, and the absolute value of the net external work does not exceed the contact node's kinetic energy loss upon impact.

When the hard kinematic contact algorithm is used, the impact is plastic, and the kinetic energy of the contacting nodes is lost. This loss is insignificant for a refined mesh, but can become significant if a coarse mesh is used. The penalty contact introduces numerical softening to the contact enforcement, analogous to adding elastic springs to the contact interface, meaning that the algorithm does not dissipate energy upon impact, as the energy stored in the springs is recoverable. 


\section{APPENDIX B:}

\section{SUPPLEMENTARY INFORMATION FOR LITERATURE REVIEW}
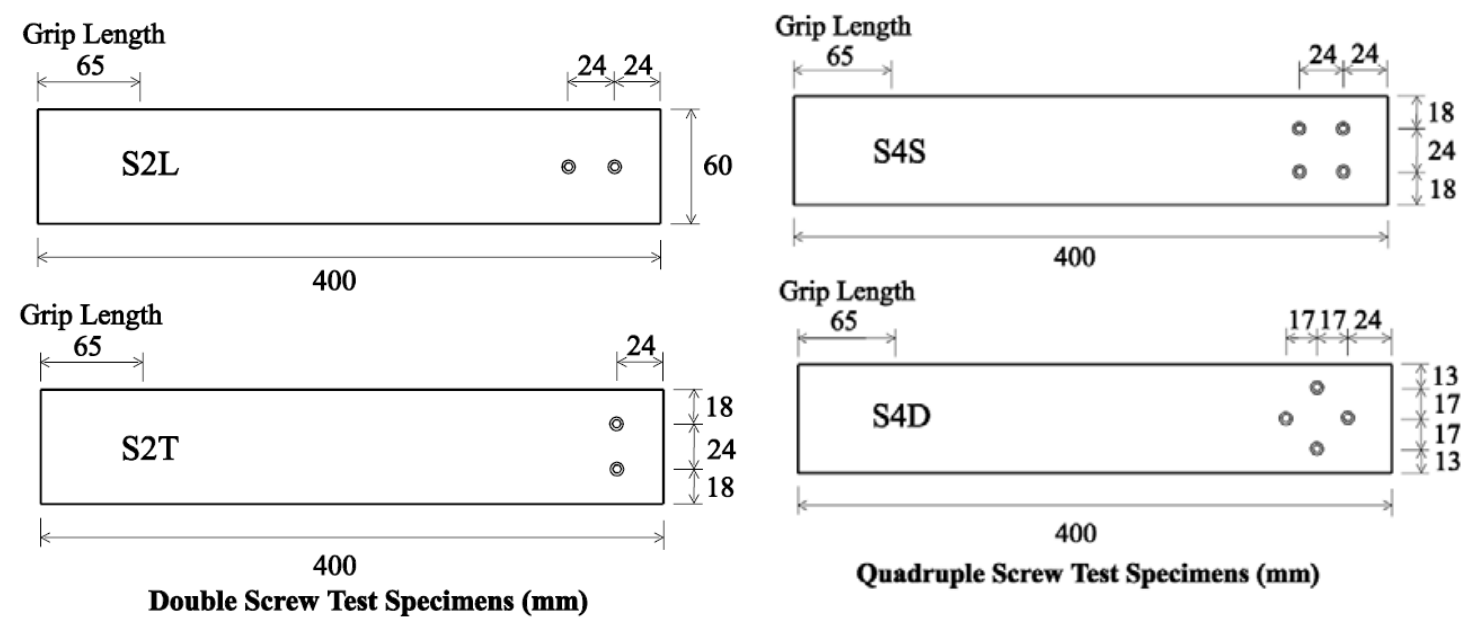

Figure B-8 Screwed connection specimens - Nominal Dimensions (Rogers \& Hancock, 1997; 1999)
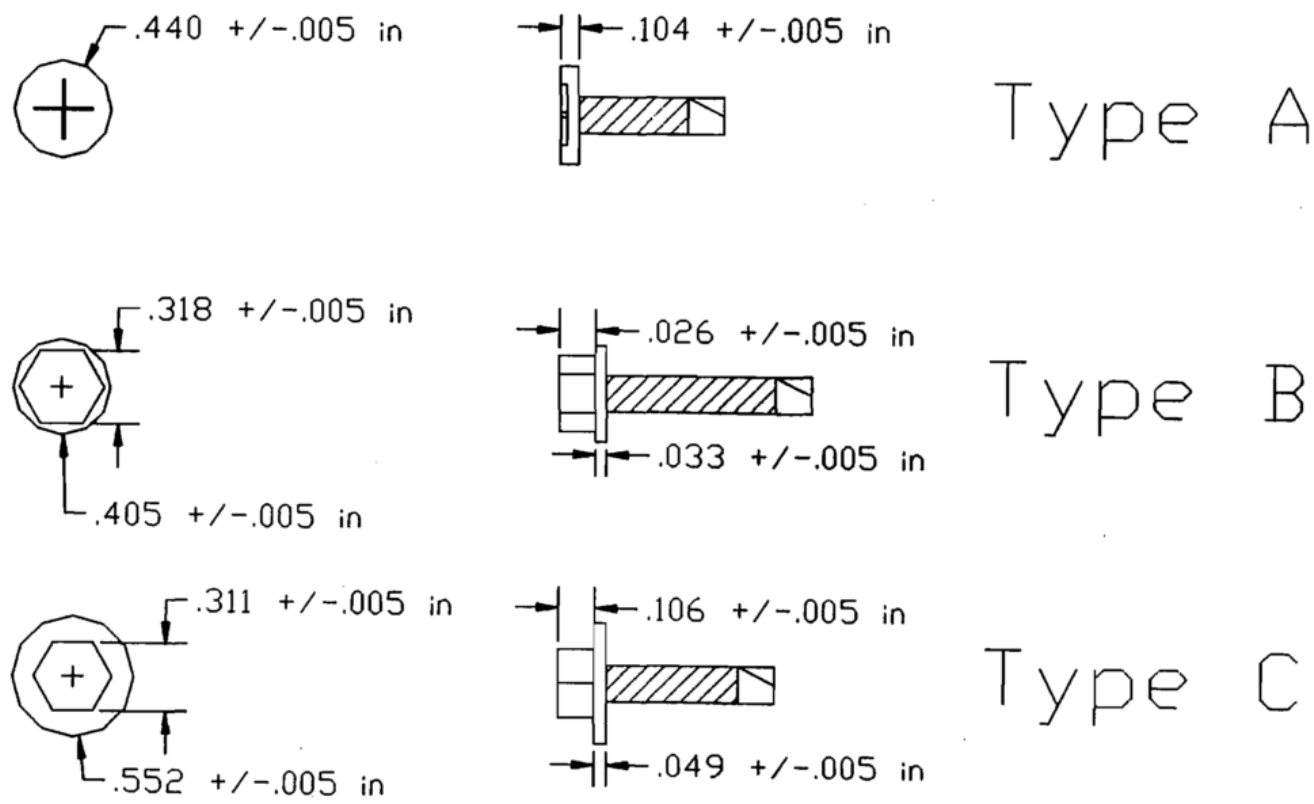

Figure B-9 Screw head descriptions (Serrette \& López, 1996) 


\section{APPENDIX C:}

\section{SUPPLEMENTARY INFORMATION FOR FINITE ELEMENT MODEL}

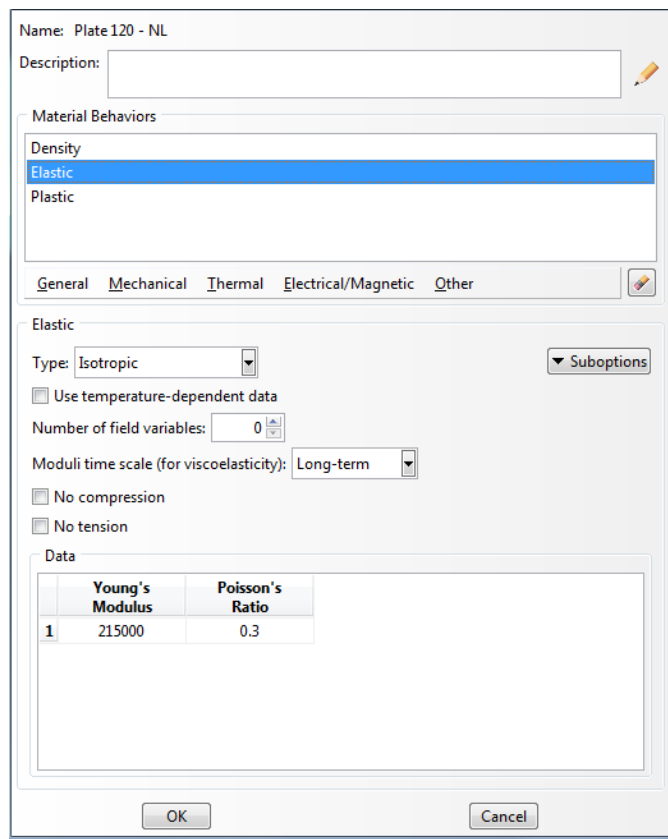

(a)

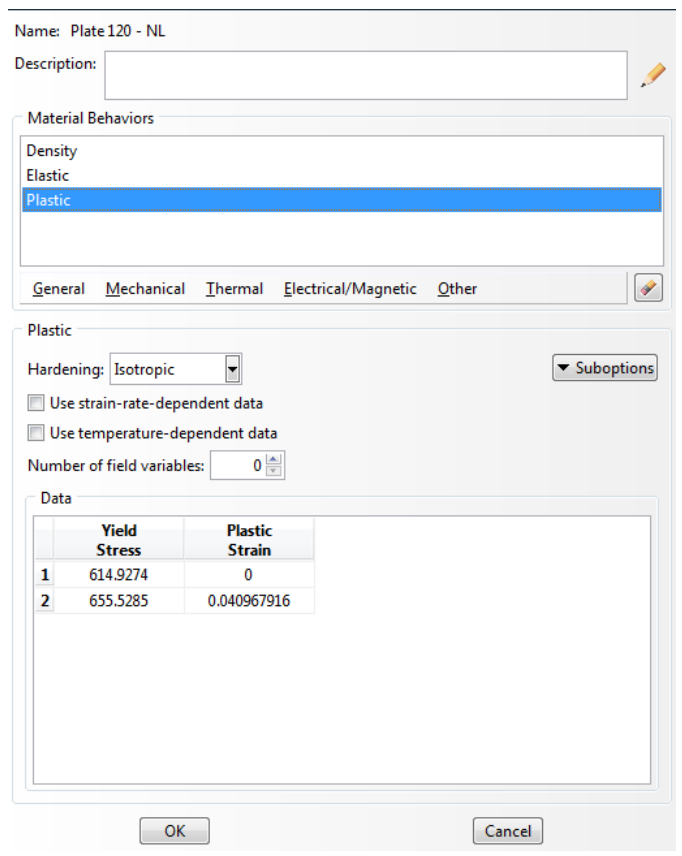

(b)

Figure C-10 Example of (a) elastic material property; and (b) plastic material property input

Name: Step-1

Type: Dynamic, Explicit

Basic Incrementation Mass scaling Other

Use scaled mass and "throughout step" definitions

from the previous step

(-) Use scaling definitions below

Data

\begin{tabular}{|c|c|c|c|c|}
\hline Region & Type & $\begin{array}{c}\text { Frequency/ } \\
\text { Interval }\end{array}$ & Factor & $\begin{array}{c}\text { Target Time } \\
\text { Increment }\end{array}$ \\
\hline $\begin{array}{l}\text { Whole } \\
\text { Model }\end{array}$ & Factor & $\begin{array}{c}\text { Beginning } \\
\text { of Step }\end{array}$ & 100 & None \\
\hline
\end{tabular}

Create... Edit... Delete

OK

Cancel

Figure C-11 Mass Scale Factor Input 


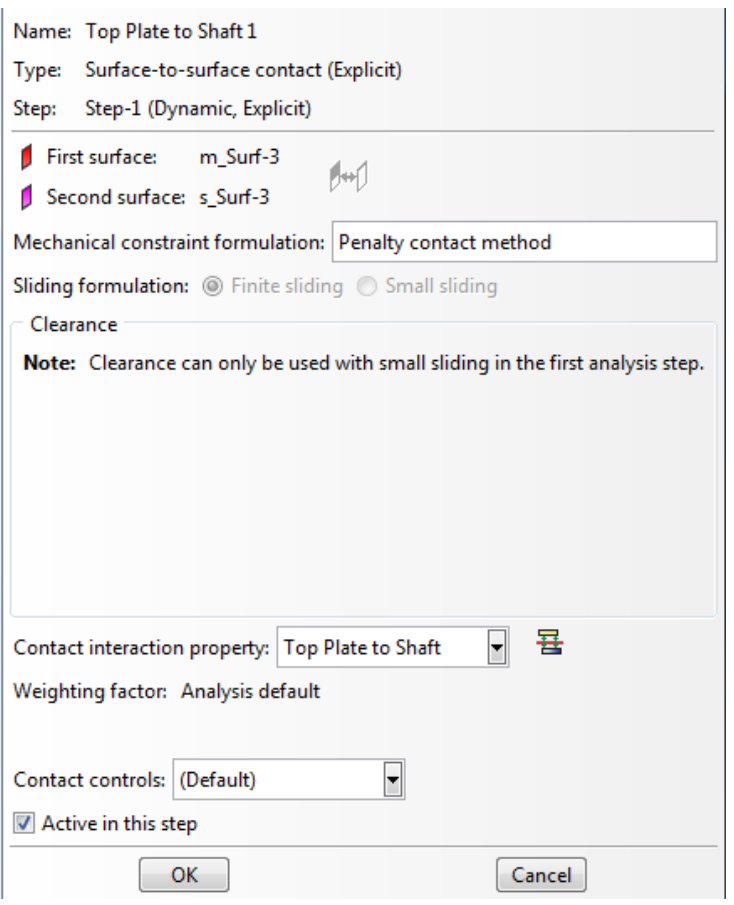

Figure C-12 Contact surface interaction definition example

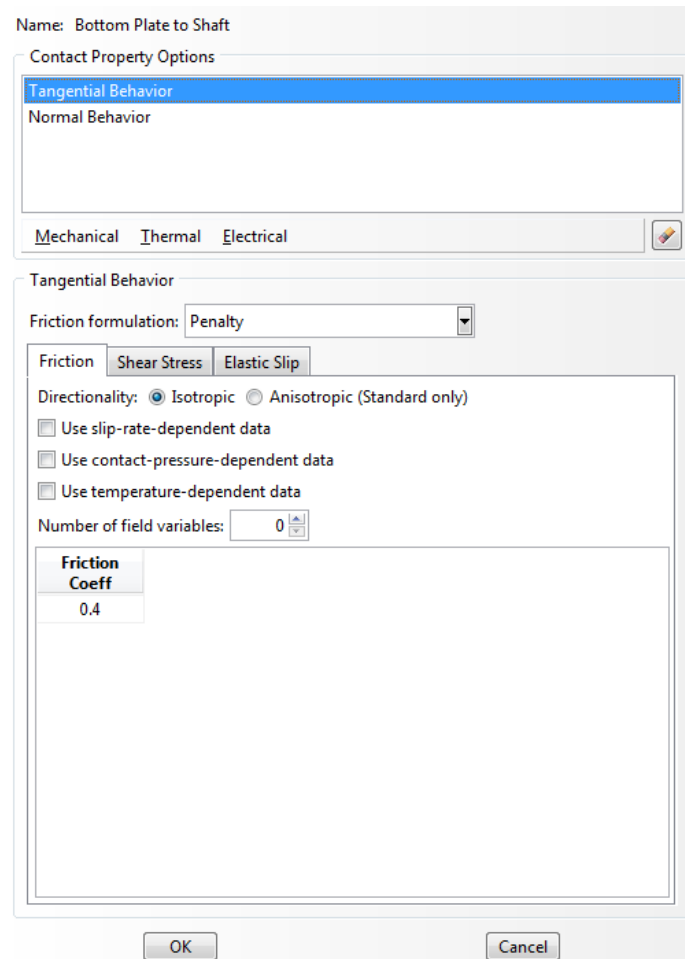

(a)

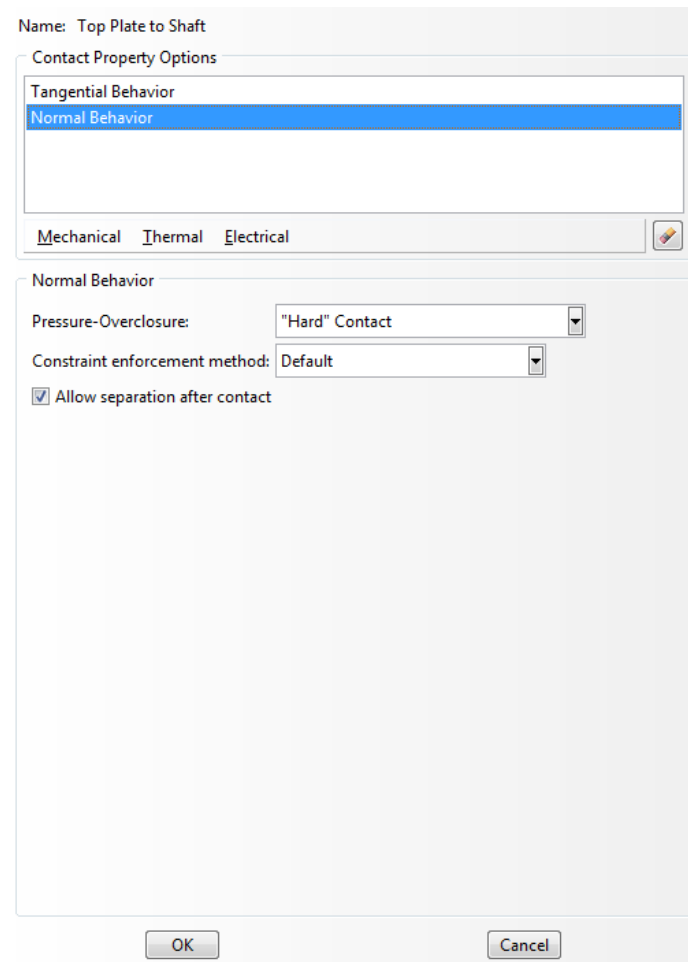

(b)

Figure C-13 Contact property definition for (a) tangential behaviour; and (b) normal behaviour example 


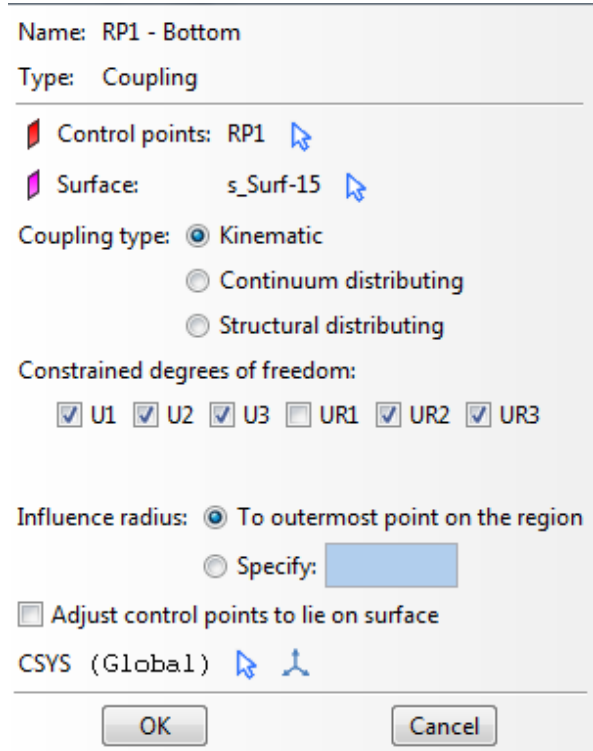

(a)

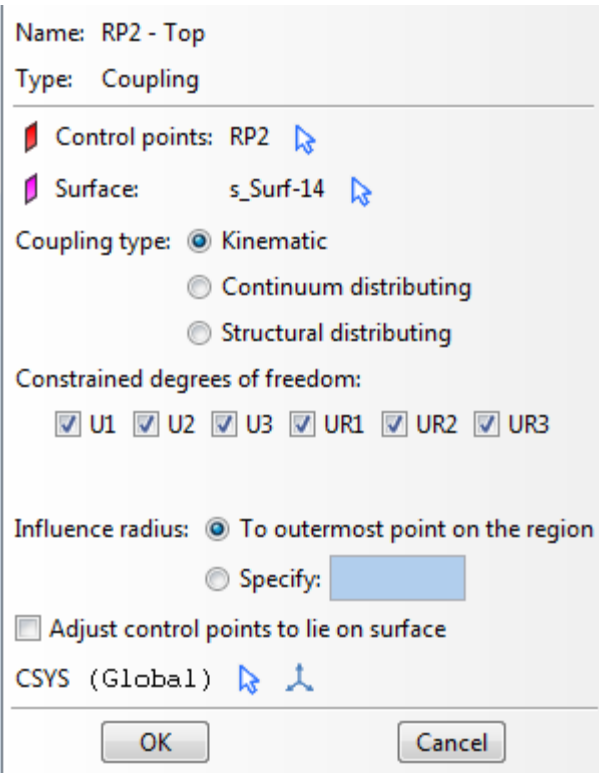

(b)

Figure C-14 Kinematic Coupling Constraint Definitions for (a) RP1 - Top Plate Edge; and (b) RP2 - Bottom Plate Edge

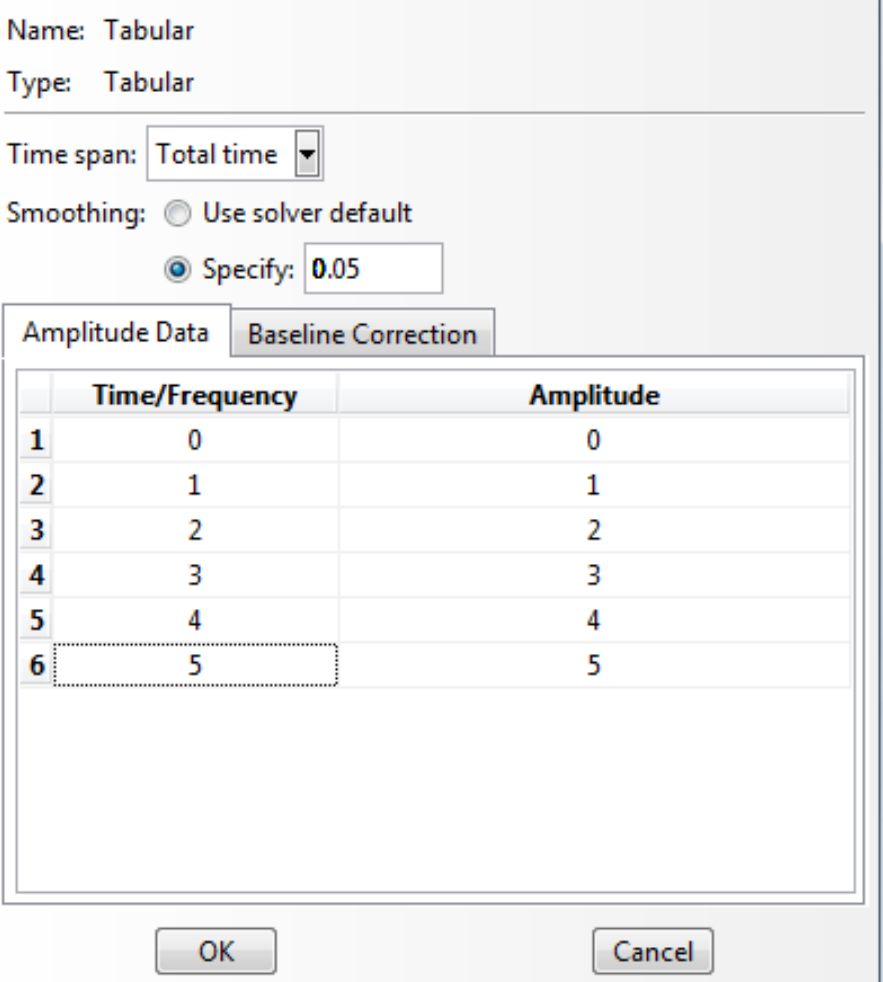

Figure C-15 Load Amplitude Definition 


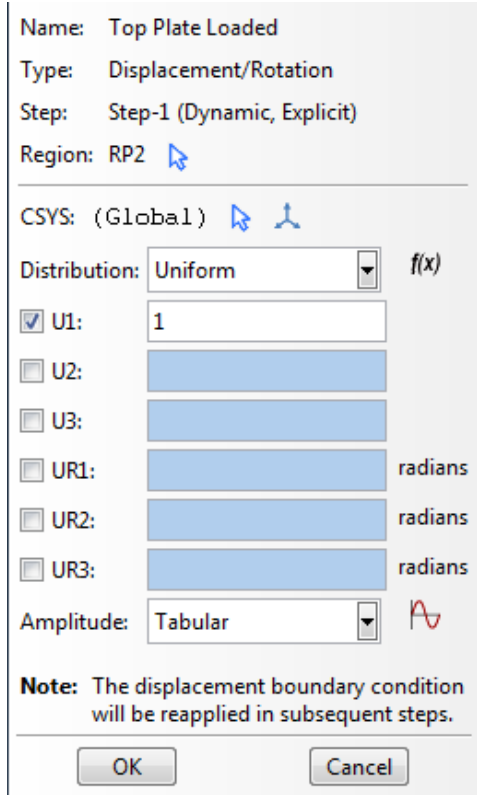

(a)

\begin{tabular}{|c|c|c|}
\hline \multicolumn{3}{|c|}{ Name: Top Plate Restrained } \\
\hline \multicolumn{3}{|c|}{ Type: Displacement/Rotation } \\
\hline \multicolumn{3}{|c|}{ Step: $\quad$ Step-1 (Dynamic, Explicit) } \\
\hline \multicolumn{3}{|l|}{ Region: RP2 } \\
\hline \multicolumn{3}{|c|}{ CSYS: (Global) } \\
\hline Distribution: 4 & Uniform & \\
\hline \multicolumn{3}{|l|}{$\square$ U1: } \\
\hline จ U2: & 0 & \\
\hline จ U3: & 0 & \\
\hline$\square$ UR1: & & radians \\
\hline$\square$ UR2: & & radians \\
\hline$\square$ UR3: & & radians \\
\hline Amplitude: & (Instantaneous) & A \\
\hline \multicolumn{3}{|c|}{$\begin{array}{l}\text { Note: The displacement boun } \\
\text { will be reapplied in subs }\end{array}$} \\
\hline OK & & \\
\hline
\end{tabular}

(b)

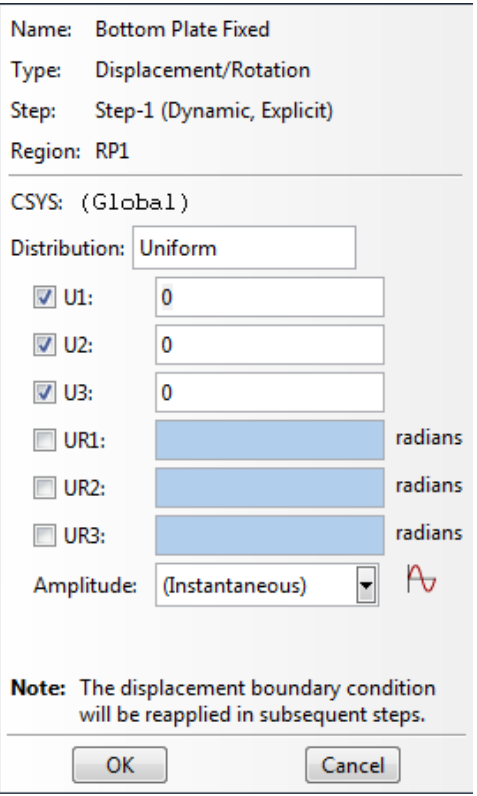

(c)

Figure C-16 Boundary Conditions as defined for the two reference points (a) Load definition for edge of top plate; (b) Restraints for edge of top plate allowing movement only in direction of loading; and (c) restraints for edge of bottom plate which is fixed against movement in all directions 


\section{APPENDIX D:}

\section{SUPPLEMENTARY INFORMATION FOR PARAMETRIC STUDY}

\section{Force-Displacement Curves for Bottom Thickness of $\mathbf{0 . 8 0} \mathbf{~ m m}$}

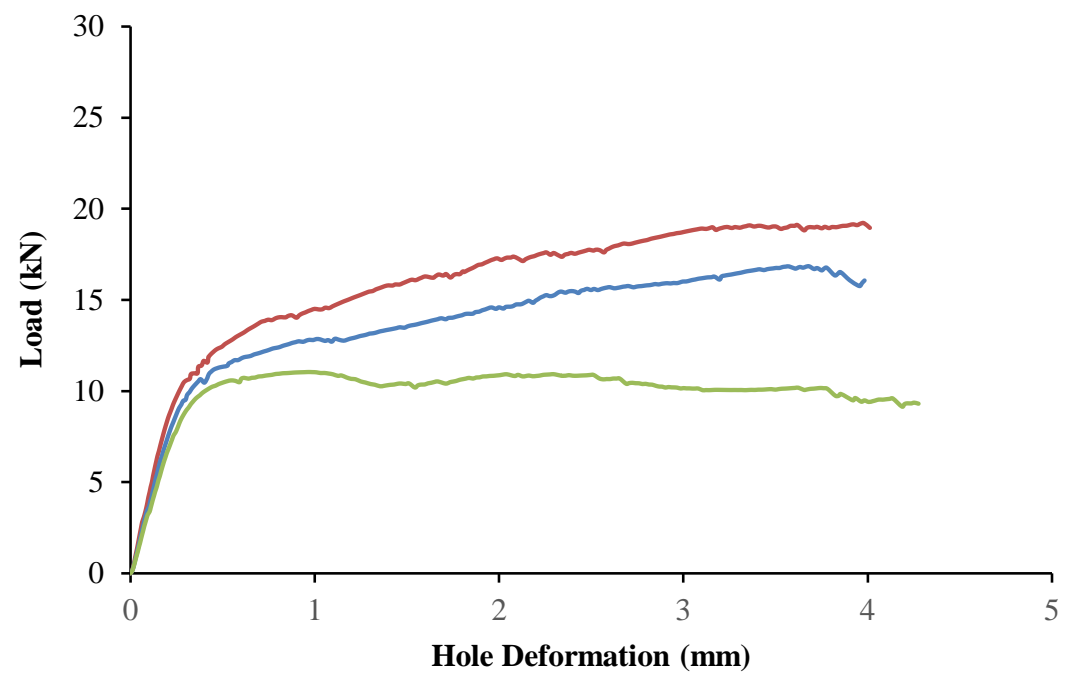

Figure D-17 Force-displacement curves for variation in sheet steel thickness with a bottom plate thickness of $0.80 \mathrm{~mm}$

Force-Displacement Curves for Bottom Thickness of $1.00 \mathrm{~mm}$

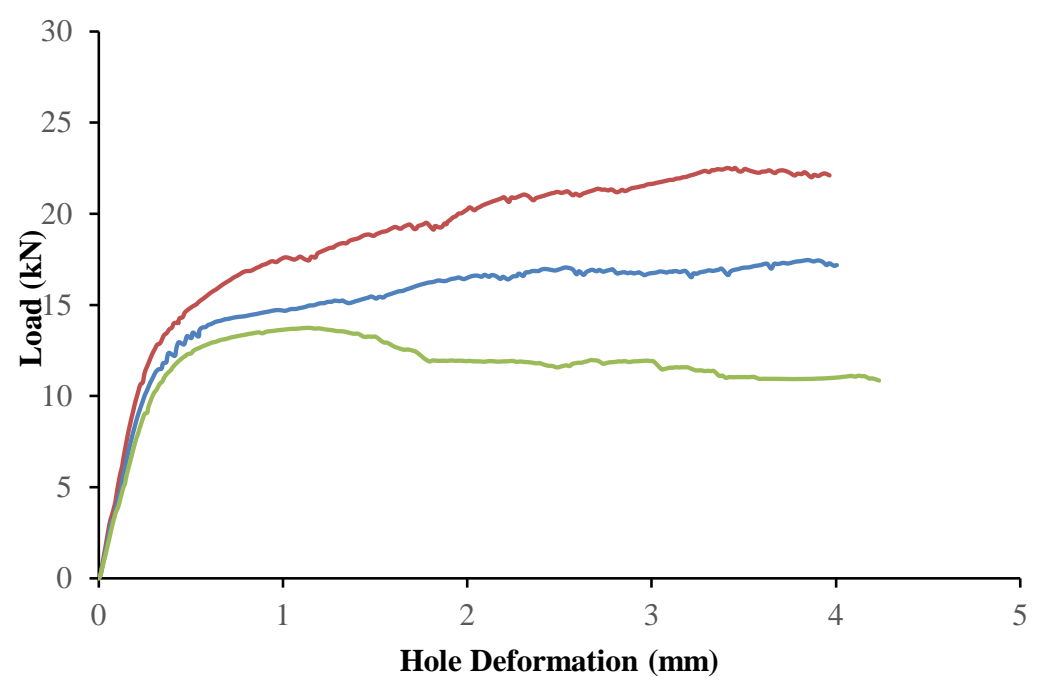

Figure D-18 Force-displacement curves for variation in sheet steel thickness with a bottom plate thickness of $1.00 \mathrm{~mm}$ 
Force-Displacement Curves for Bottom Thickness of 1.20 mm

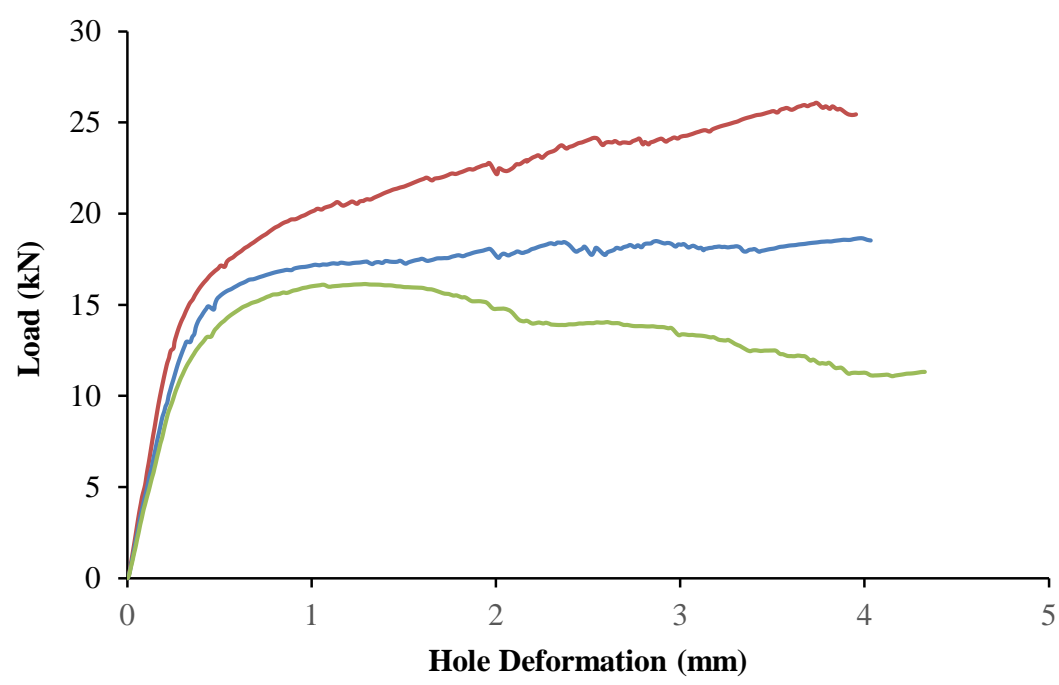

-120-300-S2-P-14

120-190-S2-P-14

120-120-S2-P-14

Figure D-19 Force-displacement curves for variation in sheet steel thickness with a bottom plate thickness of $1.20 \mathrm{~mm}$

\section{Force-Displacement Curves for Variation in Screw Size}

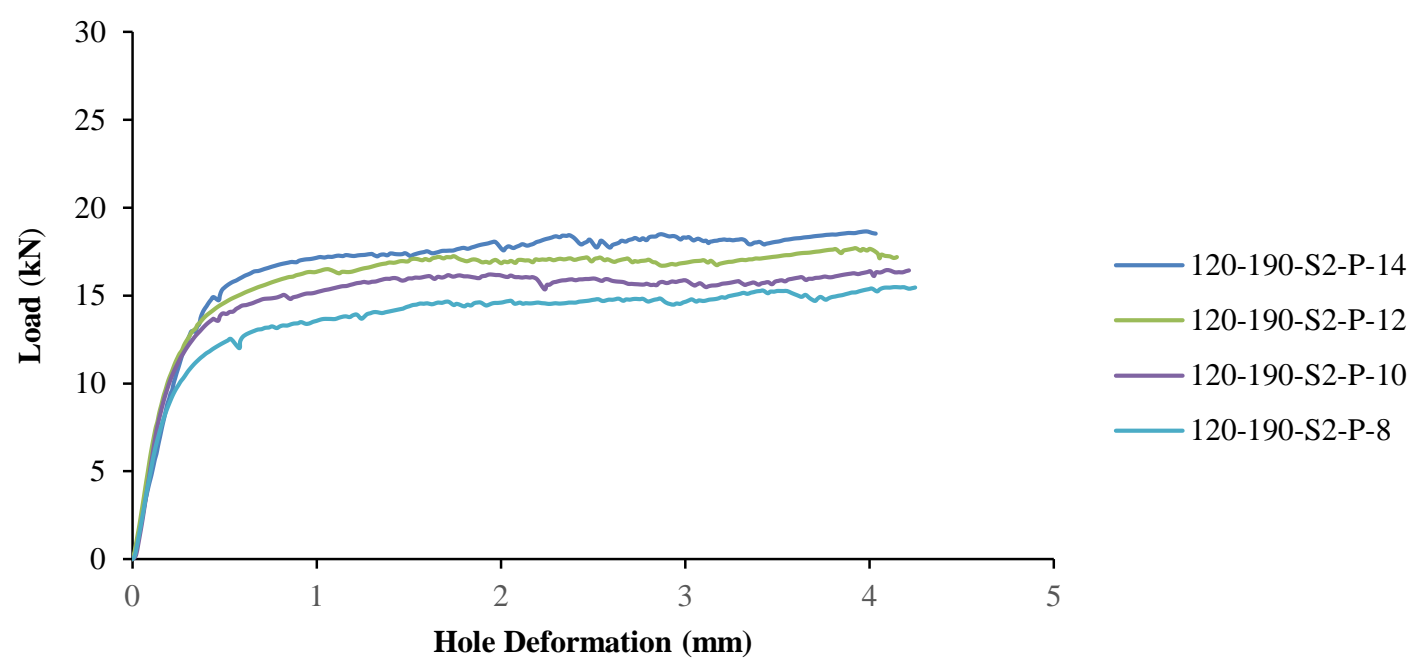

Figure D-20 Force-displacement curves for variation in screw size 